\title{
Sobre la termodinámica estadística de algunos sistemas relativistas y modelos cuánticos simples
}

\author{
Tesis que presenta \\ M. en C. Guillermo Chacón Acosta \\ Para obtener el grado de Doctor en Ciencias (Física)
}

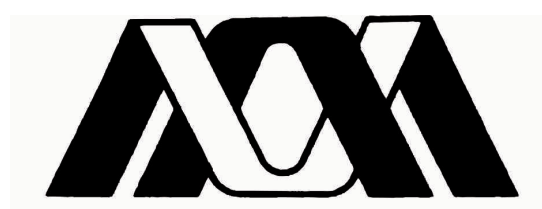

Casa abierta al tiempo

CBI

Dr. Hugo A. Morales Técotl

Coasesor
Dr. Leonardo Dagdug Lima

Coasesor 



\section{Resumen}

La incorporación de los principios de la teoría de la relatividad con los de la física estadística ha recobrado interés a la luz de experimentos y observaciones recientes en el ámbito de la física de altas energías, astrofísica y cosmología, respectivamente. Asimismo, se han realizado algunas simulaciones numéricas para investigar aspectos relativistas de variables termodinámicas. Por otro lado, teorías como la gravedad cuántica y la teoría de cuerdas, han enfrentado la necesidad de consideraciones relativistas de la física estadística.

En este trabajo se estudian algunos sistemas relativistas utilizando los métodos de la física estadística y la teoría cinética.

En primer lugar se estudia un gas simple relativista en equilibrio, en un lenguaje manifiestamente covariante, con base en la ecuación de Boltzmann. De esta manera se obtiene la función de distribución del equilibrio, conocida como distribución de Jüttner. Este análisis difiere de los existentes en la literatura en cuanto a que no se parte del uso de un marco de referencia particular. Resulta natural la introducción de un vector de Lorentz cuya norma se identifica con el recíproco de la temperatura en el marco comóvil del gas. Adicionalmente, se obtiene el correspondiente teorema de equipartición relativista.

En segundo lugar se estudian dos casos de mezclas binarias semi relativistas, cercanas al equilibrio, usando la ecuación de Boltzmann. En ambos, una de las dos especies se considera relativista mientras que la otra no. Gracias a ello el término de colisión de la ecuación de Boltzmann puede aproximarse por un término diferencial tipo Fokker-Planck. El primer caso corresponde al gas de Rayleigh, físicamente análogo al movimiento Browniano. El segundo, por otro lado, se conoce como el gas de Lorentz, y puede considerarse como el caso complementario al anterior. Para estas mezclas se obtienen expresiones para los coeficientes de viscosidad-fricción y de difusión. Además, mostramos que el gas relativista de una componente en la aproximación de colisiones rasantes, admite una descripción a través de una ecuación de Fokker-Planck covariante.

Finalmente, se estudia la termodinámica estadística en equilibrio de sistemas cuánticos simples. Estos no son relativistas, sin embargo, su cuantización se ha realizado en el esquema de lazos que es uno de los que se adopta para cuantizar a la relatividad general. Uno de los resultados de este esquema es el carácter discreto de la geometría espacial. Por esta razón se estudian las cantidades termodinámicas de los modelos mencionados. Se encuentran correcciones a estas cantidades que dependen de un parámetro que introduce el método de cuantización y que está relacionado con la escala de discretez. 



\section{Agradecimientos}

Deseo expresar mi agradecimiento a las personas que me apoyaron en la realización de esta tesis tanto de manera personal como académica. Agradezco a mis padres Mercedes y Guillermo, a mi hermano Manuel, a mi tía Beatriz, a mis abuelos Mercedes y Manuel (e. p. d.), y por supuesto a Ariadna con todo mi amor.

Quiero reconocer el respaldo brindado por CONACYT a través de la beca nacional 131138, así como al apoyo parcial del los proyectos SEP-CONACYT 51132F y CONACYT-NSF J000.359/2009, apoyos sin los cuales este trabajo no habría sido posible.

En primer lugar quiero agradecer a mis asesores Hugo A. Morales Técotl y Leonardo Dagdug Lima por su guía, lecciones y paciencia durante el desarrollo de esta tesis. Agradezco a Elisa Manrique, con quien se desarrollo parte de este trabajo, por su apoyo académico en general y por seguir aclarando muchas de mis dudas.

Asimismo, agradezco al Dr. Leopoldo García-Colín por su cuidadosa lectura de uno de los manuscritos que resultó en una publicación y en general por las muy provechosas discusiones en teoría cinética que enriquecieron el presente trabajo.

Extiendo mi agradecimiento a la Dra. Rosa María Velasco, al Profesor Gilberto M. Kremer, Abel Camacho Quintana, Hernando Quevedo, Ana Laura García Perciante, Alfredo Sandoval Villalbazo, David Cubero, Roberto Sussman, Tonatihu Matos, Merced Montesinos, Alberto Güijosa, Marco Maceda, Eloy Ayón, Jerónimo Cortéz, José Antonio Zapata y Alejandro Corichi, así como a Elias Castellanos, William Cuervo y Ernesto Flores por sus muy valiosos comentarios y sugerencias. También doy las gracias a Héctor Hugo Hernández Hernández, Mercedes Velázquez y Yuri Bonder por sus opiniones y apoyo personal.

Además deseo agradecer la hospitalidad, valiosos comentarios y enseñanzas de los profesores Martin Reuter y Martin Bojowald durante las estancias en la Universidad Johannes Gutenberg en Mainz, Alemania en 2008 y en la Universidad Estatal de Pennsylvania, Estados Unidos en 2010, respectivamente.

G. C. A.

México D. F., Febrero 2011. 



\section{Índice general}

$\begin{array}{ll}\text { 1. Introducción } & 1\end{array}$

2. Ecuación de Boltzmann Relativista $\quad 7$

2.1. Teoría Cinética y Ecuación de Boltzmann Relativista . . . . . . . . . . . . . . . . . . . 7

2.2. Sección Transversal para la dispersión elástica Relativista . . . . . . . . . . . . . . . 12

2.3. Conflicto de las interacciones entre partículas relativistas y la ecuación de Boltzmann . . . 15

3. Análisis manifiestamente covariante de un gas simple relativista $\quad 17$

3.1. Introducción . . . . . . . . . . . . . . . . . . . . . . . . . . . . 18

3.2. Distribución de Jüttner . . . . . . . . . . . . . . . . . . . . . . . . 21

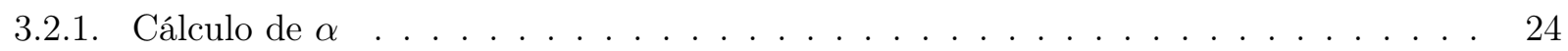

3.2.2. $\Theta^{\mu}$ y la Temperatura invariante . . . . . . . . . . . . . . . 26

3.3. Momento de un Gas relativista . . . . . . . . . . . . . . . . . . . . . . . . . 28

3.4. Equipartición relativista . . . . . . . . . . . . . . . . . . . . . 33

3.5. Discusión . . . . . . . . . . . . . . . . . . . . . . . . 37

4. Ecuación de Fokker-Planck relativista:

Mezclas binarias semirelativistas y gas simple $\quad 45$

4.1. Introducción . . . . . . . . . . . . . . . . . . . . . . . . 4 46

4.2. Ecuación de Boltzmann relativista para una mezcla binaria . . . . . . . . . . . . . . 46

4.3. Gas de Rayleigh - Movimiento Browniano relativista . . . . . . . . . . . . . . . . . . 48

4.4. Ecuación de Fokker-Planck para el gas de Lorentz relativista . . . . . . . . . . . . . . . . 52

4.5. Ecuación de Fokker-Planck manifiestamente covariante para el gas simple . . . . . . . . 56

4.6. Discusión . . . . . . . . . . . . . . . . . . . . . . . . . . . . 60 
5. Termodinámica estadística de sistemas simples cuantizados por lazos 65

5.1. Introducción . . . . . . . . . . . . . . . . . . . . . . . . 66

5.2. Representación por lazos de la Mecánica Cuántica ． . . . . . . . . . . . . . . . . . . 68

5.3. Eigenvalores del Hamiltoniano Polimérico . . . . . . . . . . . . . . . . . . . . . . . 73

5.3.1. Oscilador Armónico . . . . . . . . . . . . . . . . . . . . . . . . . 73

5.3.2. Partícula en una caja . . . . . . . . . . . . . . . . . . . 74

5.4. Correcciones poliméricas a magnitudes termodinámicas de sistemas simples . . . . . . . 77

5.4.1. Ensamble de osciladores armónicos cuantizados por lazos . . . . . . . . . . . . . 77

5.4.2. Función de partición para el Gas Ideal polimérico . . . . . . . . . . . . . . . . 82

5.5. Discusión . . . . . . . . . . . . . . . . . . . . . . . . . . 90

6. Conclusiones y Perspectivas $\quad 95$

A. La Ecuación de Boltzmann Relativista como una aproximación a la teoría cuántica de $\begin{array}{ll}\text { campos } & 101\end{array}$

A.1. Dinámica de los campos . . . . . . . . . . . . . . . . . . . . . . . . . . . . . 101

A.2. La Función de Wigner . . . . . . . . . . . . . . . . . . . . . . . . . . . . . . . 102

A.3. Ecuación de Transporte Relativista . . . . . . . . . . . . . . . . . . . . . . . . 105

B. Partículas Relativistas Interactuantes 111

B.1. Teorema de No - Interacción de Partículas Relativistas . . . . . . . . . . . . . . . . . . . 111

$\begin{array}{lr}\text { C. Funciones de Bessel modificadas } & 117\end{array}$

C.1. Primera solución $I_{n}(z) \ldots \ldots \ldots \ldots \ldots \ldots \ldots \ldots \ldots$

C.2. Segunda solución $K_{n}(z) \ldots \ldots \ldots \ldots \ldots$

D. Integrales de colisión para las aproximaciones de Fokker-Planck 121

D.1. Integrales para el movimiento Browniano relativista . . . . . . . . . . . . . . . . . . . . 121

D.2. Integrales para el gas de Lorentz relativista . . . . . . . . . . . . . . . . . . . . 124

E. Gravedad Cuántica de Lazos $\quad 127$

E.1. El problema de cuantizar la gravedad . . . . . . . . . . . . . . . . . . . . . . 127

E.2. Gravedad Cuántica de Lazos . . . . . . . . . . . . . . . . . . . . . . . . . . . . . . . 129

E.2.1. Preliminares . . . . . . . . . . . . . . . . . . . . . . . . . . . . 129

E.2.2. Geometría Cuántica . . . . . . . . . . . . . . . . . 130

E.2.3. Cosmología Cuántica de Lazos . . . . . . . . . . . . . . . . . . . . 132

$\begin{array}{lr}\text { Bibliografía } & 139\end{array}$ 


\section{Introducción}

Es posible afirmar que la teoría cinética relativista comenzó con la derivación de la función de distribución de equilibrio para un gas relativista simple por F. Jüttner en 1911 seguida de su versión cuántica en 1928, a través de un procedimiento de maximización de la entropía [1]. En 1935 Walker da el siguiente paso hacia la descripción del gas relativista encontrando la ecuación de evolución que debe satisfacer la función de distribución, en el caso sin colisiones. La generalización relativista de la ecuación de Boltzmann incluyendo colisiones fue dada por Lichnerowicz y Marrot en 1940 [2, 3]. Desde entonces se han hecho generalizaciones de los métodos estándares de la teoría cinética.

Uno de los propósitos de la teoría cinética es derivar leyes macroscópicas con base en las ecuaciones de evolución microscópicas. Con estas ideas en mente Marrot y Taub [4] fueron los primeros en mostrar que las leyes de conservación de masa y de energía-momento pueden obtenerse para el gas relativista. En su libro [5], Synge describe los resultados más importantes para gases relativistas en equilibrio e introduce la notación vectorial 4-dimensional. La década de 1960 fue muy importante y fértil para la teoría cinética relativista. A principios de los 60's Israel [6] y Kelly [7], entre otros autores, adaptaron los métodos de Chapmann-Enskog y Grad al dominio de la relatividad. Israel también estudió generalizaciones a sistemas estándar como las partículas de Maxwell. Con estos métodos se pudieron calcular los coeficientes de transporte para el gas relativista a partir de la ecuación de Boltzmann covariante. Una de las consecuencias más importantes en este caso es que el gas relativista tiene viscosidad volumétrica a diferencia del caso del gas no relativista. Este resultado tiene importantes repercusiones, por ejemplo en el efecto de la viscosidad de los neutrinos en la evolución del universo, el estudio de la formación de galaxias, estrellas de neutrones, etc. La ecuación de Boltzmann relativista incluyendo el efecto de campos gravitacionales fue escrita primero por Chernikov [8] y Lindquist [9]. Posteriormente, Stewart [10] hace una descripción más general de la teoría en espacios curvos. Es en este trabajo, junto con el de Ehlers [11], donde se construye el formalismo matemático de la teoría cinética en el contexto de la relatividad general. 
En su libro de 1980 [12] de Groot, van Leeuwen y van Weert estudian la deducción de la ecuación de Boltzmann a partir de la dinámica subyacente a un sistema de partículas cuánticas relativistas; esta dinámica es proporcionada por la teoría cuántica de campos. En ese trabajo se calculan los coeficientes de transporte para sistemas específicos que tienen un papel importante en astrofísica y cosmología. Sin embargo, no estudian los efectos del campo gravitacional. En un trabajo más reciente Cercignani y Kremer [13], además de una revisión de la teoría cinética relativista, estudian mezclas de gases relativistas en donde ocurren reacciones químicas o nucleares y se analiza la propagación de ondas de choque en un gas relativista. En este trabajo se siguen los esquemas presentados en los textos [12] y [13].

En la literatura hay distintas presentaciones de la teoría cinética relativista dependiendo el enfoque que se siga. Por ejemplo, se han propuesto alternativas a la distribución de Jüttner [14, 15, 16, 17]. Incluso hay algunos intentos por establecer los fundamentos de la termodinámica irreversible relativista sobre la base de una teoría cinética relativista de primer orden [18]. Recientemente se desarrollaron simulaciones numéricas de dinámica molecular para el gas relativista que indican que la distribución de Jüttner es en realidad la generalización relativista correcta de la distribución del equilibrio [19, 20, 22, 23].

La teoría cinética relativista tiene una extensa gama de aplicaciones. Históricamente, entre las primeras se encuentra el trabajo de Chandrasekhar [24], quien desarrolló la teoría de la estructura estelar para estrellas en equilibrio y en estado estacionario. En cosmología al estudiar las épocas tempranas en la vida del universo es necesario considerar ciertos procesos disipativos. Por ejemplo, la época del desacoplamiento entre la radiación y la materia. En [25], Bernstein estudia la ecuación de Boltzmann en un fondo de Friedman-Lemaître-Robertson-Walker, esta métrica describe un modelo de Universo en expansión, homogéneo e isotrópico [26]. Se encuentra que no existe solución de equilibrio para la función de distribución de un gas en esa situación. Por otro lado las reacciones durante la época de la nucleosíntesis ocurren fuera de equilibrio. En el universo temprano las especies que existían (neutrinos, fotones, electrones y positrones) eran todas relativistas. Es clara la necesidad de una teoría cinética relativista para investigar la evolución e interacciones entre las especies en esa época. De alguna forma, la mayor parte de la teoría cosmológica surge de resolver la ecuación de Boltzmann para el universo en expansión con fotones, neutrinos y materia oscura [26], [27], [28]. Se ha estudiado también la distorsión del espectro de cuerpo negro del Fondo Cósmico de Microondas (CMB), producida por la dispersión de Compton de los fotones del CMB por electrones de los cúmulos de galaxias, conocida como el efecto Sunyaev-Zeldovich [31], [32]. Ese proceso afecta la distribución de la radiación. Este efecto permite calcular parámetros cosmológicos como la constante de Hubble, el corrimiento al rojo, etc. Este efecto se ha intentado explicar a través de ecuaciones de difusión relativistas como la ecuación de Kompaneetz [33].

Las aplicaciones en astrofísica son muy diversas. Por ejemplo, en el estudio de los jets o chorros relativistas (flujos de plasmas colimados con velocidades cercanas a la de la luz), modelos para los destellos de rayos gamma, efectos disipativos producidos por la presencia de vorticidad en estrellas de neutrones, entre otros [29], [30]. 
Recientemente se han realizado experimentos de física nuclear basados en colisiones de iones pesados a altas velocidades, tales como el RHIC (Relativistic Heavy Ion Collider) o el LHC (Large Hadron Collider) para describir el plasma de quarks y gluones [34, 35]. Las especies involucradas en estos experimentos son relativistas y por lo tanto es necesaria una base teórica para calcular los coeficientes de transporte correspondientes de estos sistemas.

Incorporar los principios de la relatividad con los de la teoría cinética no sólo es importante en el contexto de las anteriores aplicaciones, es crucial para entender los fundamentos teóricos de la descripción de sistemas relativistas de muchos cuerpos, donde no es claro cómo definir una variable temporal natural [10]. En termodinámica clásica no relativista, la descripción de los sistemas en equilibrio no está ligada con el movimiento del sistema mismo. Sin embargo, cuando extendemos el análisis al caso relativista especial, la descripción desde cualquier marco de referencia se vuelve imprescindible. Por ejemplo, en [36] se comienza a generalizar las leyes de la termodinámica a partir de principios de simetría y conservación y se observa la necesidad de incluir al movimiento del sistema.

Existen marcos teóricos donde se pueden estudiar los fundamentos de la termodinámica, tal es el caso de la formulación generalmente covariante de la mecánica estadística [37]. Esta es una aplicación de los métodos de la mecánica estadística a sistemas invariantes ante reparametrizaciones temporales. Se demuestra que incluso en ausencia de los conceptos de tiempo y energía, los métodos estadísticos pueden aplicarse apareciendo más parámetros que caracterizan el equilibrio ${ }^{1}$. Otro ejemplo es la Geometrotermodinámica [38], que utiliza las herramientas de la geometría diferencial para abordar problemas en termodinámica, particularmente la termodinámica de hoyos negros [39].

Se sabe de la teoría cuántica de campos en espacio-tiempo curvo, que puede asociarse una entropía a los agujeros negros, la entropía de Bekenstein-Hawking. La teoría cuántica de campo en espacio-tiempo curvo implica que los hoyos negros emiten radiación, la llamada radiación de Hawking, se conjetura que esta radiación los llevará a evaporarse. Esto conduce a varias preguntas. En física estadística la entropía está relacionada con el número de microestados a que el sistema tiene acceso, sin embargo, en el caso de los agujeros negros ¿cuáles son los microestados asociados con su entropía? Esta es una pregunta que algunos candidatos a teorías de gravedad cuántica han intentado responder. La gravedad cuántica intenta implementar de manera consistente las interacciones gravitacionales dentro del marco de la teoría cuántica [40]. En algunas propuestas existe una escala de longitud mínima, posiblemente la longitud de Planck, que presumiblemente es una escala fundamental en la que aparece una microestructura al espaciotiempo. Existen otras propuestas que buscan revelar los efectos de tal longitud mínima, por ejemplo los conjuntos causales [41], o la gravedad estocástica [42], ambos esquemas utilizan métodos propios de la termodinámica fuera de equilibrio y procesos de transporte. Finalmente la evaporación de un hoyo negro debe considerarse como un sistema fuera de equilibrio termodinámico, por lo que el estudio cinético

\footnotetext{
${ }^{1} \mathrm{Al}$ escoger una variable temporal y por tanto la energía se puede ver que los parámetros extra están relacionados con el movimiento del sistema
} 
relativista de estos sistemas es indispensable.

En este punto puede apreciarse que el estudiar la estadística y termodinámica de sistemas relativistas, es de suma importancia tanto en aspectos aplicados como fundamentales. Para concluir esta lista se añadirán un par de resultados recientes. Uno de los resultados importantes más reciente en teoría de cuerdas o teoría $\mathrm{M}$, es la llamada correspondencia AdS-CFT que es la equivalencia entre una teoría de campos conforme y una teoría con gravedad (supergravedad o teoría de cuerdas) en una dimensión mayor. En particular se ha hecho la descripción hidrodinámica de ambos sistemas, conocida como correspondencia fluido-gravedad, dando muy buenos resultados [43]. Dentro de este escenario basado en el principio holográfico hay una reciente discusión sobre la naturaleza misma de la gravedad, si ésta es una fuerza emergente en lugar de ser fundamental [44]. Estas discusiones, y el hecho bien conocido de que la gravedad pueda describirse como un sistema termodinámico [45], refuerzan el interés por estudiar la termodinámica y la teoría cinética relativistas.

El propósito del presente trabajo es investigar con base en la física estadística varios sistemas relativistas. Estos incluyen el gas simple, mezclas binarias cercanas al equilibrio, así como sistemas simples cuantizados de manera similar a la cuantización por lazos de la gravedad.

La tesis está estructurada de la siguiente forma. En el capítulo 2 se hace una revisión de la ecuación básica en teoría cinética relativista, es decir, de la ecuación de Boltzmann relativista. Se hace énfasis en el problema de la no interacción entre partículas relativistas clásicas, el cual implica un obstáculo en el estudio de sistemas de muchos cuerpos, particularmente en la ecuación de Boltzmann que contiene un término de interacción donde aparece la sección recta relacionada con el potencial de interacción entre partículas. Este aparente problema queda resuelto si se considera a la ecuación de Boltzmann como una aproximación de las ecuaciones de la teoría cuántica de campos.

En el capítulo 3 se presenta una derivación alternativa de la función de distribución de equilibrio para un gas simple relativista teniendo control de las propiedades de transformación de las cantidades termodinámicas involucradas. En particular se hace un análisis manifiestamente covariante donde es necesario introducir un vector de Lorentz cuya norma resulta ser proporcional al inverso de la temperatura comóvil del sistema. Con este vector se analiza el momento relativista total del sistema y una versión manifiestamente covariante del teorema de equipartición de la energía que en particular se reduce a resultados previos encontrados en la literatura.

El capítulo 4 está dedicado al estudio de mezclas binarias compuestas por una especie relativista y una no relativista. Además, una de ellas tiene una densidad muy pequeña en comparación con la otra, esto permite hacer ciertas aproximaciones en la integral de colisión de la correspondiente ecuación de Boltzmann que describe al sistema, pudiéndose escribir ésta como un operador diferencial tipo Fokker-Planck. Se estudian las extensiones relativistas de los sistemas conocidos como el gas de Rayleigh, físicamente análogo al movimiento Browniano, y el gas de Lorentz. Estos sistemas pueden considerarse físicamente complementarios debido a sus características. Adicionalmente se encuentran los coeficientes de fricción-viscosidad y 
de difusión para ambos sistemas. Los coeficientes correspondientes al gas de Rayleigh concuerdan con resultados conocidos en el límite no relativista. En el caso del gas de Lorentz aparece un coeficiente asociado a un tiempo de relajación que multiplica a la función de distribución en la ecuación diferencial respectiva. Además, se hace un análisis del gas de una componente en la aproximación de pequeña transferencia de momento y colisiones rasantes, que permite una descripción manifiestamente covariante del término de Fokker-Planck que reemplaza a la integral de colisión, en este caso también se obtienen los coeficientes de transporte y una generalización covariante relativista de la relación de fluctuación-disipación de Einstein. Motivados por las potenciales aplicaciones de los métodos estadísticos a las diversas propuestas a la gravedad cuántica, exploramos posibles efectos que los métodos alternativos de cuantización pueden inducir en un análisis termoestadístico. Especialmente en la gravedad cuántica de lazos, que es un enfoque a la gravedad cuántica independiente del fondo, aparece una escala mínima de longitud. El esquema de cuantización resulta ser no equivalente a la cuantización usual de Schrödinger en mecánica cuántica. En el capítulo 5 aplicamos esta cuantización alternativa a sistemas simples con número finito de grados de libertad y estudiamos las modificaciones que esta induce a las correspondientes cantidades termodinámicas obtenidas del análisis mecánico estadístico usual. Nuestros resultados se asemejan a resultados obtenidos en la literatura de la fenomenología de la gravedad cuántica.

Finalmente en el capítulo 6 donde se discuten las conclusiones y resultados de la tesis y se plantean perspectivas de trabajo futuro. Se adjuntan además cinco apéndices. El primero contiene la aproximación de las ecuaciones de campo, a través de la función de Wigner, para obtener la ecuación de Boltzmann. En el segundo se repasa el teorema de no interacción de partículas relativistas. En el tercer apéndice se repasan propiedades generales de las funciones de Bessel modificadas. En el cuarto apéndice se presentan los cálculos de las integrales de colisión utilizadas en el capítulo 4. En el último apéndice se presentan los diversos enfoques a la gravedad cuántica, especialmente se revisan algunos aspectos de la gravedad cuántica de lazos.

Como parte de este trabajo se realizaron las siguientes publicaciones:

- [46] G. Chacón-Acosta, L. Dagdug and H. Morales-Técotl, Manifestly covariant Jüttner distribution and equipartition theorem, Phys. Rev. E 81, 021126 (2010).

- [47] G. Chacón-Acosta, L. Dagdug and H. A. Morales-Técotl, On the Fokker-Planck equation for the relativistic Lorentz gas, in New Trends in Statistical Physics: Festschrift in Honor of Leopoldo García-Colín's 80th Birthday, ed. A. Macías and L. Dagdug, (pp. 275-292 World Scientific, 2010).

- [48] G. Chacón-Acosta, L. Dagdug and H. Morales-Técotl, Relativistic Momentum and Manifestly Covariant Equipartition Theorem Revisited, in Gravitational Physics: Testing Gravity from Submillimeter to Cosmic Scale, ed. H. Morales-Técotl, L. A. Ureña-López, R. Linares-Romero and H. H. García-Compean, AIP Conf. Proc. 1256, 231-238 (2010). 
- [49] G. Chacón-Acosta, L. Dagdug and H. Morales-Técotl, A covariant Fokker-Planck equation for a simple gas from relativistic kinetic theory, in IV Mexican Meeting on Theoretical and Experimental Physics: Relativistic Fluids and Biological Physics, Ed. L. Dagdug, A. L. Garcia-Perciante, A. Sandoval-Villalbazo, L. S. Garcia-Colin, AIP Conf. Proc. 1312, 73-79 (2010).

- [50] G. Chacón-Acosta, E. Manrique, L. Dagdug and H. Morales-Técotl, Statistical thermodynamics of simple polymer quantum systems, (en preparación). 


\section{Ecuación de Boltzmann Relativista}

El principal objetivo de la teoría cinética de los gases es obtener una descripción macroscópica (hidrodinámica, termodinámica) de un sistema a partir de sus constituyentes microscópicos haciendo promedios estadísticos de muchas partículas, de las cantidades físicas relevantes como la energía el momento etc.

En este capítulo se revisa la generalización a la relatividad especial de la teoría cinética, basada principalmente en la obtención heurística de la ecuación de Boltzmann relativista que es una ecuación integrodiferencial para la función de distribución de una partícula, con esta distribución se calculan los promedios con los que se obtienen las cantidades termodinámicas.

Asimismo se revisa el teorema de no interacción para partículas relativistas el cual representa un problema para la derivación heurística de la ecuación de Boltzmann basada en interacciones binarias elásticas. Este problema se resuelve al considerar a la teoría cinética relativista como una aproximación de la teoría cuántica de campo.

\subsection{Teoría Cinética y Ecuación de Boltzmann Relativista}

Desde el punto de vista macroscópico el estado de un sistema formado por una colección de $\mathcal{N}$ partículas de masa $m$, se describe a través de su densidad de número de partículas, la densidad de energía, la velocidad hidrodinámica y la densidad de entropía.

En el caso relativista se introduce un 4-vector de Lorentz conocido como el 4-flujo de partículas:

$$
N^{\mu}=n \mathcal{U}^{\mu}
$$

donde $\mathcal{U}^{\mu}$ es la 4-velocidad hidrodinámica del fluido, $n$ es la densidad de número de partículas en el marco en co-móvil y las componentes espaciales corresponden al flujo de partículas. Recordemos que el índice $\mu$ toma valores 0 la componente temporal y 1,2,3 las componentes espaciales. La ecuación (2.1) es la definición de Eckart de la velocidad hidrodinámica $\mathcal{U}^{\mu}$ de los fluidos relativistas [51], sin embargo hay que 
decir que existen varias posibles definiciones sobre todo en el caso fuera de equilibrio [12], [52].

En lugar de considerar únicamente la densidad de energía, existe un tensor de rango 2 llamado el tensor de energía-momento del fluido $T^{\mu \nu}$, las componentes de este tensor corresponden a las siguientes cantidades:

$T^{00}=$ Densidad de energía,

$T^{0 i}=c^{-1} \times$ Flujo de energía a través de la superficie con normal en dirección $i$,

$T^{i 0}=c \times$ Densidad de momento $p^{i}$,

$T^{i j}=$ Flujo de momento $p^{i}$ a través de la superficie con normal en dirección $j$.

Además su traza espacial se identifica con la presión del fluido:

$$
\mathcal{P} \equiv-\frac{1}{3} \Delta_{\mu \nu} T^{\mu \nu}
$$

donde $\Delta^{\mu \nu} \equiv \eta^{\mu \nu}-\frac{1}{c^{2}} \mathcal{U}^{\mu} \mathcal{U}^{\nu}$ y $\eta^{\mu \nu}$ la métrica de Minkowski. La cantidad definida en (2.2) usualmente tiene dos contribuciones, una correspondiente a la parte fuera de equilibrio de la traza de $T^{\mu \nu}$, conocida como presión dinámica y la presión de equilibrio o presión hidrostática.

Una característica importante de este tensor es que es simétrico, componentes con índices intercambiados son iguales $T^{\alpha \beta}=T^{\beta \alpha} . T^{0 i}=T^{i 0}$ no son sino nombres distintos de la misma cantidad física (dada la relación entre masa y energía). No es difícil convencerse de que la simetría de $T^{i j}$ se debe a la estabilidad mecánica rotacional de los elementos de fluido ${ }^{1}$. La parte sin traza de este tensor puede escribir se como:

$$
\sigma^{\mu \nu}=\left(\Delta_{\sigma}^{\mu} \Delta_{\rho}^{\nu}-\frac{1}{3} \Delta_{\sigma \rho} \Delta^{\mu \nu}\right) T^{\sigma \rho}
$$

este se conoce como tensor de esfuerzos cortantes o tensor viscoso.

El tensor de energía-momento provee una descripción covariante de la energía y el momento del fluido. Es importante mencionar que el tensor de energía-momento que se considera toma en cuenta únicamente la energía cinética y la energía en reposo de las partículas. La suposición de que el sistema es diluido implica que la energía de interacción de las partículas es muy pequeña comparada con su energía cinética, de otra forma $T^{\mu \nu}$ incluiría un término potencial.

Ambas cantidades $N^{\mu}$ y $T^{\mu \nu}$ se identifican con los momentos estadísticos de la función de distribución correspondiente. Por otra parte es necesario introducir una función que cumpla la versión relativista del teorema $H$ de Boltzmann, así definimos un 4-vector de Lorentz $S^{\mu}$ conocido como 4-flujo de entropía

$$
S^{\mu}=n s \mathcal{U}^{\mu}
$$

La ecuación de Boltzmann es una ecuación para el comportamiento espacio-temporal de la función de distribución de una partícula. Para poder tener una ecuación cerrada para la distribución hay que hacer

\footnotetext{
${ }^{1}$ Ver discusión en el capítulo correspondiente del libro de Schutz [54].
} 
algunas suposiciones: primeramente que el gas es suficientemente diluido para permitir únicamente interacciones binarias y que la distribución varíe lentamente tanto en el tiempo como en el espacio, además de la suposición estadística conocida como caos molecular que implica que después de cada encuentro las partículas permanecen no correlacionadas entre $\mathrm{si}^{2}$.

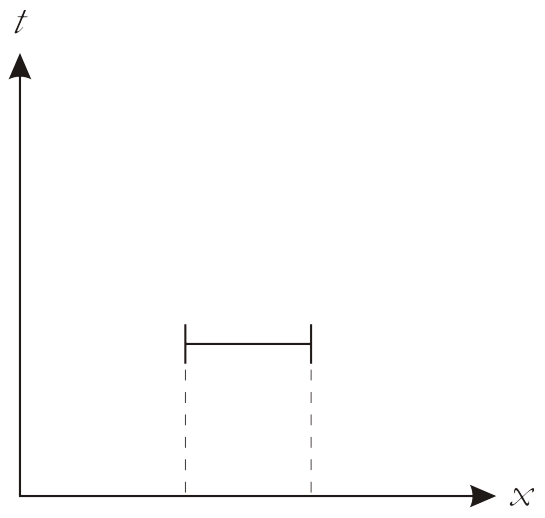

(a)

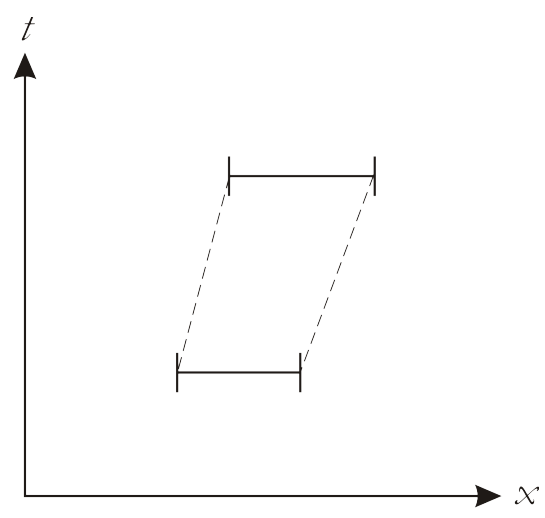

(b)

Figura 2.1: (a) Diagrama espacio-temporal donde se representa el volumen del sistema de $\mathcal{N}$ partículas al tiempo inicial. (b) Evolución temporal del sistema.

Entonces consideremos un sistema formado por $\mathcal{N}$ partículas de masa $m$. La representación 4-dimensional de las condiciones iniciales de este sistema de partículas puede esquematizarse en un diagrama de espaciotiempo como un segmento horizontal que representa el volumen del sistema al tiempo inicial (fig. 2.1 (a)). Como se sabe en este diagrama una partícula se caracteriza por su línea de mundo. De esta manera el conjunto de las líneas de mundo de las partículas del sistema forman un tubo llamado tubo de mundo, el cual por lo tanto, simboliza su historia (fig. 2.2).

Considérese ahora un elemento de 3-superficie en el espacio-tiempo el cual está caracterizado por un vector normal $\hat{n}^{\mu}$, donde $d \mathbf{S}^{\mu}=\hat{n}^{\mu} d^{3} S$. Al ser $N^{\mu}$ el flujo de partículas en el espacio-tiempo, entonces el número de líneas de mundo que cruzan la 3-superficie diferencial se obtiene

$$
d N_{L M}=\frac{1}{c} N^{\mu} \hat{n}_{\mu} d^{3} S=f(x, p) p^{\mu} \hat{n}_{\mu} d^{3} S \frac{d^{3} p}{p^{0}},
$$

donde $c^{-1}$ en la primera igualdad se introduce para tener las unidades adecuadas. Esta expresión se puede interpretar como el número de líneas de mundo que atraviesan la hipersuperficie $d \mathbf{S}^{\mu}$ cuyos momentos caen dentro de un ángulo sólido $d \Omega_{p}=d^{3} p / p^{0}$ alrededor de $p^{\mu}$. Notemos que la componente temporal corresponde a la energía de la partícula $p^{0}=E / c$ y que la medida en $(2.5) d^{3} p / p^{0}$ es una medida invariante [53]. Eligiendo coordenadas tales que el vector unitario únicamente tenga componente temporal $\hat{n}^{\mu} \rightarrow(1,0,0,0)$, la 3-superficie será completamente espacial $d^{3} S=d^{3} x$. De esta manera la ecuación (2.5)

\footnotetext{
${ }^{2} \mathrm{Al}$ final de este capítulo se comenta una derivación de la ecuación de transporte con base en la teoría cuántica de campo la cual sólo pide ausencia de correlaciones al inicio en lugar que varias veces a lo largo del tiempo. Ver apéndice A.
} 


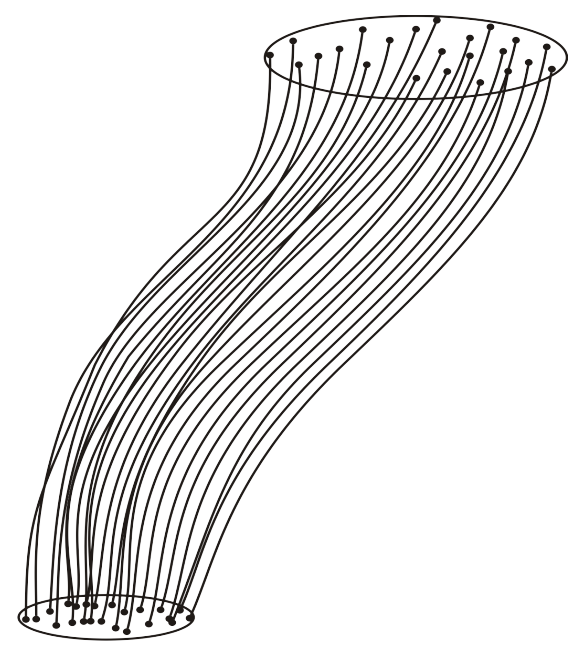

Figura 2.2: Tubo de mundo que representa las historias de las partículas que forman el sistema.

se reduce a

$$
d N_{L M}=f(x, p) p^{0} \hat{n}_{0} d^{3} x \frac{d^{3} p}{p^{0}}=f(x, p) d^{3} x d^{3} p .
$$

En el límite de bajas velocidades esta ecuación se reduce al número de partículas en un volumen $d^{3} x$, en un intervalo de momentos $d^{3} p$. Esto implica que la función de distribución relativista consistentemente se reduce a la no relativista cuando se considera una hipersuperficie espacial y en el límite de bajas velocidades.

Supongamos ahora que se tiene una hipersuperficie $S$ que sea frontera de una región espacio-temporal $\mathcal{V}_{\mathrm{x}}=d^{4} x$, es decir, $S=\partial \mathcal{V}_{\mathrm{x}}$. Integrando (2.5) sobre la hipersuperficie $S$ y sobre un intervalo espacial de momentos $\Delta^{3} p$ se tiene

$$
N_{L M}=\int_{S} \int_{\Delta^{3} p} f(x, p) p^{\mu} \hat{n}_{\mu} d^{3} S \frac{d^{3} p}{p^{0}}
$$

esta integral nos da el número total de líneas de mundo que cruzan la 3-superficie $S$ y tienen momento en el intervalo $\Delta^{3} p$. Por lo tanto, también es el número de líneas de mundo que salen (ó entran) del volumen espacio-temporal $\mathcal{V}_{\mathrm{x}}$. Si la región $S$ es la hipersuperficie del tubo de mundo de las partículas conformado por las superficies $S_{1}, S_{2}$ y $S_{3}$ como se muestra en la figura 2.3, entonces aplicando el teorema de Gauss relativista ${ }^{3}$ a la ecuación (2.7) se obtiene

$$
\int_{\partial \mathcal{V}_{\mathrm{x}}} \int_{\Delta^{3} p} f p^{\mu} \hat{n}_{\mu} d^{3} S \frac{d^{3} p}{p^{0}}=\int_{\mathcal{V}_{\mathrm{x}}} \int_{\Delta^{3} p} \partial_{\mu} f p^{\mu} d^{4} x \frac{d^{3} p}{p^{0}}
$$

Es claro que cuando no hay ninguna interacción entre las partículas, estas no pueden salir de la región $\mathcal{V}_{\mathrm{x}}$, por lo tanto el lado izquierdo de la ecuación (2.7) es $N_{L M}=0$.

\footnotetext{
${ }^{3}$ Una demostración sencilla puede hallarse en el libro de Schutz [54], para un tratamiento más amplio del teorema de la divergencia en relatividad ver el libro de Synge [55].
} 


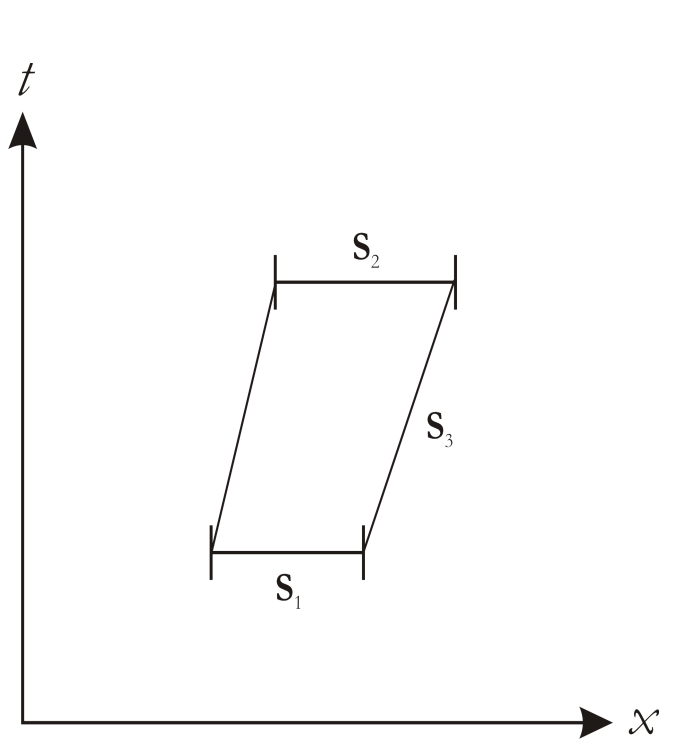

(a)

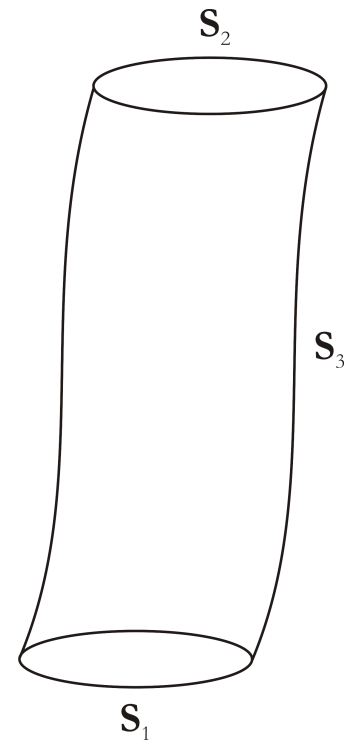

(b)

Figura 2.3: Hipersuperficie del tubo de mundo $S=S_{1}+S_{2}+S_{3}$.

Suponiendo arbitrarios los intervalos espacio-temporales $\mathcal{V}_{\mathrm{x}} \mathrm{y}$ de momentos $\Delta^{3} p$ la ecuación (2.8) es equivalente a

$$
p^{\mu} \partial_{\mu} f=p^{\mu} \frac{\partial f}{\partial x^{\mu}}=\frac{d f}{d \lambda}=0
$$

con $\lambda=\tau / m$ un parámetro afín y $\tau$ el tiempo propio de una de las partículas. Esta es la ecuación relativista de Boltzmann en el caso sin colisiones.

Considerando un intervalo espacio-temporal $\Delta^{4} x$ y en el espacio de momentos $\Delta^{3} p$, el número de partículas en estos intervalos se ve afectado por las colisiones entre partículas. Este cambio puede escribirse como

$$
\Delta^{4} x \frac{\Delta^{4} p}{p^{0}} \mathcal{C}(x, p)
$$

donde $\mathcal{C}(x, p)$ es una función invariante conocida como término de colisión. Consideraremos únicamente procesos de colisión entre dos partículas que inicialmente tienen momento $p^{\mu}$ y $p_{1}^{\mu}$ antes del encuentro y $p^{\prime \mu}$ y $p_{1}^{\prime \mu}$, después de la colisión. Para obtener (2.10) es necesario contar las líneas de mundo que entran y salen de un elemento de volumen espacio-temporal $\Delta^{4} x$, es decir contando los choque entre las partículas con momento $p^{\mu}$ y $p_{1}^{\mu}$ y con momento $p^{\prime \mu}$ y $p_{1}^{\prime \mu}$, que hacen que las partículas cambien su momento de tal forma que el valor final de éste se encuentre o salga del rango $\Delta^{3} p$. Este número resulta ser proporcional al número de partículas correspondiente a cada valor del momento involucrado, es decir, $f d^{3} p f_{1} d^{3} p_{1}$, y al intervalo espacio-temporal $d^{4} x$; el factor de proporcionalidad restante se conoce como tasa de transición $W\left(p, p_{1} \mid p^{\prime}, p_{1}^{\prime}\right)$, la cual sólo depende de los momentos entrantes y salientes de las partículas y es un invariante relativista, además $W$ estará determinada por la dinámica que obedezcan las partículas ${ }^{4}$. Dado

\footnotetext{
${ }^{4}$ Como se verá más delante esta cantidad se identificará con la sección transversal de dispersión. Una forma sencilla de
} 
que se supone que $f$ varia lentamente en $x^{\mu}$, la diferencia de posiciones espacio-temporales antes y después de las colisiones puede despreciarse, es por eso que la tasa de transición no depende de esta cantidad.

De esta forma el número total de partículas que dejan el elemento de volumen espacio temporal por interacciones entre ellas se obtiene integrando sobre el rango de todos los momentos y restando aquellas partículas que salen de las que entran, tal que se obtiene

$$
\mathcal{C}(x, p)=\int \frac{d^{3} p_{1}}{p_{1}^{0}} \frac{d^{3} p^{\prime}}{p^{\prime} 0} \frac{d^{3} p_{1}^{\prime}}{p_{1}^{\prime 0}}\left[f^{\prime} f_{1}^{\prime} W\left(p^{\prime}, p_{1}^{\prime} \mid p, p_{1}\right)-f f_{1} W\left(p, p_{1} \mid p^{\prime}, p_{1}^{\prime}\right)\right]
$$

esto es precisamente $\mathcal{C}(x, p)$. Utilizando (2.10) y (2.9) podemos escribir la ecuación de transporte de Boltzmann relativista incluyendo el término de colisión

$$
p^{\mu} \partial_{\mu} f=p^{\mu} \frac{\partial f}{\partial x^{\mu}}=\int \frac{d^{3} p_{1}}{p_{1}^{0}} \frac{d^{3} p^{\prime}}{p^{\prime} 0} \frac{d^{3} p_{1}^{\prime}}{p_{1}^{\prime 0}}\left[f^{\prime} f_{1}^{\prime} W\left(p^{\prime}, p_{1}^{\prime} \mid p, p_{1}\right)-f f_{1} W\left(p, p_{1} \mid p^{\prime}, p_{1}^{\prime}\right)\right] .
$$

Enseguida se identificará la tasa de transición con la sección transversal de un proceso de dispersión.

\subsection{Sección Transversal para la dispersión elástica Relativista}

$\mathrm{Al}$ ser $W\left(p, p_{1} \mid p^{\prime}, p_{1}^{\prime}\right)$ un invariante relativista tiene que ser independiente del marco de referencia donde se mida. Al depender únicamente de los 4-momentos de las partículas antes y después de la colisión, la forma funcional de $W$ debe depender de los diez invariantes que pueden formarse combinando los 4momentos $p^{\mu}, p_{1}^{\mu}, p^{\prime \mu}, p_{1}^{\prime}$. Se debe excluir la norma de cada vector que corresponde a la masa en reposo de cada partícula $p^{\mu} p_{\mu}=m^{2} c^{2}$ ya que no son cantidades variables. Las seis cantidades restantes no son independientes debido a la ley de conservación del 4-momento

$$
p^{\mu}+p_{1}^{\mu}=p^{\prime \mu}+p_{1}^{\prime \mu}
$$

dadas las restricciones anteriores y combinaciones algebraicas entre ellas [57], los invariantes independientes son en realidad sólo dos que podrían ser $p^{\mu} p_{\mu}^{\prime}$ y $p^{\mu} p_{1 \mu}^{\prime}$, ya que se demuestra son independientes [57]. Sin embargo, normalmente es útil introducir las llamadas variables de Mandelstam como las variables invariantes independientes

$$
\begin{aligned}
s & =\left(p+p_{1}\right)^{2} \\
t & =\left(p-p^{\prime}\right)^{2} \\
u & =\left(p-p_{1}^{\prime}\right)^{2}
\end{aligned}
$$

donde una de las variables no es independiente. La interpretación de estas variables es la siguiente: $s$ es el cuadrado de la energía en el sistema de referencia del centro de momento, $t$ es el cuadrado

verificar que es invariante puede verse en [13] y [56], donde se construye una forma invariante del número de colisiones en un marco particular y luego se generaliza para cualquiera. 
de la transferencia de momento, mientras que para $u$ no hay una interpretación física clara. Al no ser independientes estas variables cumplen con la relación

$$
s+t+u=4 m^{2} c^{2} .
$$

La variable $t$ puede relacionarse con al ángulo de dispersión $\cos \chi$ en el sistema de centro de momento que se define como sigue

$$
\cos \chi=\frac{\left(p^{\mu}-p_{1}^{\mu}\right)\left(p_{\mu}^{\prime}-p_{1 \mu}^{\prime}\right)}{\left(p-p_{1}\right)^{2}}
$$

de forma tal que la relación entre $t$ y $\chi$ es la siguiente

$$
\begin{aligned}
s & =4\left(m^{2} c^{2}+P^{2}\right), \\
t & =2 P^{2}(\cos \chi-1),
\end{aligned}
$$

donde $P$ es el 4-momento total del sistema $P^{\mu}=p^{\mu}+p_{1}^{\mu}$. De forma tal que $2 t /\left(s-4 m^{2} c^{2}\right)=\cos \chi-1$ que viene de combinar (2.14)-(2.16) y (2.17) dando $t=-s+2 m^{2} c^{2}$.

Finalmente llegamos a la conclusión de que la amplitud de transición $W\left(p, p_{1} \mid p^{\prime}, p_{1}^{\prime}\right)$ es función de los 2 invariantes $s$ y $t$, donde alguno de ellos puede sustituirse por el ángulo de dispersión $\chi$ y por tanto puede escribirse de la siguiente forma

$$
W\left(p, p_{1} \mid p^{\prime}, p_{1}^{\prime}\right)=s \sigma(s, \chi) \delta^{4}\left(p+p_{1}-p^{\prime}-p_{1}^{\prime}\right),
$$

donde la distribución delta toma en cuenta la conservación del 4-momento y $\sigma(s, \chi)$ es una función general de la energía y el ángulo de dispersión, al tener dimensiones de área se identificará con la sección transversal de dispersión. El flujo es igual al producto de las densidades de las partículas por la velocidad relativa que puede expresarse en términos del flujo invariante como $c F / p^{0} p_{1}^{0}$, donde el flujo invariante $F$ puede expresarse en términos de las variables de Mandelstam como

$$
F=\frac{1}{2} \sqrt{s\left(s-4 m^{2} c^{2}\right)}
$$

De esta forma la sección de dispersión puede escribirse como

$$
\Delta \sigma=\int \sigma(s, \chi) d \Omega=\int \frac{d^{3} p^{\prime}}{p^{\prime} 0} \frac{d^{3} p_{1}^{\prime}}{p_{1}^{\prime 0}} \frac{W\left(p, p_{1} \mid p^{\prime}, p_{1}^{\prime}\right)}{F},
$$

donde $\Omega$ es el ángulo sólido en el sistema del centro de momento. Al sustituir la propuesta (2.21) en (2.23) corroboraremos que la función en (2.21) es realmente la sección transversal de dispersión:

$$
\Delta \sigma=\int \frac{d^{3} p^{\prime}}{p^{\prime}} \frac{d^{3} p_{1}^{\prime}}{p_{1}^{\prime 0}} \frac{s \sigma(s, \chi)}{F} \delta^{4}\left(p+p_{1}-p^{\prime}-p_{1}^{\prime}\right),
$$

integrando en $\mathbf{p}_{1}^{\prime}$ y considerando (2.14) y (2.19) obtenemos

$$
\Delta \sigma=\int d^{3} p^{\prime} \frac{\sigma(s, \chi)}{F} \delta\left(\frac{1}{2} \sqrt{s}-p^{\prime 0}\right)
$$


si utilizamos que

$$
d^{3} p^{\prime}=\left|\mathbf{p}^{\prime}\right|^{2} d\left|\mathbf{p}^{\prime}\right| d \Omega, \quad \delta\left(\frac{1}{2} \sqrt{s}-\sqrt{\left|\mathbf{p}^{\prime}\right|^{2}+m^{2} c^{2}}\right)=\sqrt{\frac{s}{s-4 m^{2} c^{2}}} \delta\left(\left|\mathbf{p}^{\prime}\right|-\frac{1}{2} \sqrt{s-4 m^{2} c^{2}}\right),
$$

es posible entonces hacer la integración en la magnitud del momento espacial $\left|\mathbf{p}^{\prime}\right|$, esto demuestra que $\sigma$ es realmente la sección transversal de dispersión.

La expresión propuesta para la amplitud de transición (2.21) tiene algunas propiedades de simetría deseables, por ejemplo, es claro que $W$ es simétrica ante el intercambio $p, p_{1} \rightarrow p^{\prime}, p_{1}^{\prime}$, tal que

$$
W\left(p, p_{1} \mid p^{\prime}, p_{1}^{\prime}\right)=W\left(p^{\prime}, p_{1}^{\prime} \mid p, p_{1}\right),
$$

que usualmente se conoce como propiedad de balance detallado.

Es interesante notar que, a diferencia del caso no relativista, esta tasa de transición no requiere de la suposición de invariancia ante reflexiones espacio-temporales del proceso de dispersión correspondiente, es decir, no es necesario suponer la colisión inversa. Las propiedades de simetría de $W$ vienen del hecho de que es un invariante de Lorentz. Es importante recordar que en el caso no relativista la colisión inversa existe únicamente para ciertos caso particulares, como para partículas puntuales. Como se verá más adelante la invariancia de $W$ el caso relativista en está garantizada desde la perspectiva de la teoría cuántica de $\operatorname{campos}^{5}$.

En particular utilizando la propiedad de balance detallado (2.27) en la ecuación de transporte (2.12), podemos factorizar $W$; si además sustituimos la expresión (2.21) y realizamos las integrales de los momentos primados hasta el ángulo sólido obtenemos la expresión estándar para la ecuación de Boltzmann relativista [12], [13]

$$
p^{\mu} \partial_{\mu} f=p^{\mu} \frac{\partial f}{\partial x^{\mu}}=\int \frac{d^{3} p_{1}}{p_{1}^{0}} d \Omega\left(f^{\prime} f_{1}^{\prime}-f f_{1}\right) F \sigma .
$$

Si consideramos fuerzas externas habría que sumar al lado izquierdo un término de la forma $m \partial_{\mu}\left(\mathcal{K}^{\mu} f\right)$. Es interesante notar que la ecuación manifiestamente covariante (2.28) puede escribirse en notación $3+1$ dimensional, en cuyo caso sería

$$
\frac{\partial f}{\partial t}+v^{i} \frac{\partial f}{\partial x^{i}}+\frac{\partial\left(f \mathrm{~F}^{i}\right)}{\partial p^{i}}=\int\left(f^{\prime} f_{1}^{\prime}-f f_{1}\right) g_{\varnothing} \sigma d \Omega d^{3} p_{1},
$$

donde $v^{i}$ son las componentes espaciales de la velocidad de las partículas, $\mathrm{F}^{i}=m c \mathcal{K}^{i} / p^{0}$, es la fuerza ordinaria en el marco comóvil, la cantidad $g_{\varnothing}$ se conoce como velocidad relativa de Møller y está relacionada con el flujo invariante $g_{\varnothing}=c F /\left(p^{0} p_{1}^{0}\right)$, en el límite de bajas velocidades tiende a la velocidad relativa entre las partículas, y la ecuación se reduce a la ecuación de Boltzmann no relativista.

Curiosamente (2.29) es idéntica a la versión no relativista de la ecuación de Boltzmann, el único lugar donde intervienen los efectos relativistas es en el producto $g_{\varnothing} \sigma$, que está relacionado con la relación entre velocidad y momento en relatividad y con las interacciones entre partículas codificadas en la sección transversal de dispersión $\sigma,[12]$.

\footnotetext{
${ }^{5}$ Ver apéndice $\mathrm{A}$ ec. (A.14)
} 


\subsection{Conflicto de las interacciones entre partículas relativistas y la ecuación de Boltzmann}

El hecho de que las correcciones relativistas entren precisamente en el término que codifica las interacciones es algo controversial, ya que existe un resultado conocido como el teorema de no-interacción de partículas relativistas [58], [59], [60], que se resume en el apéndice B, y cuyo resultado principal es que las partículas relativistas no pueden interactuar sin la intervención de un campo, esto implicaría que no es posible definir la sección transversal de colisión y por tanto la ecuación de Boltzmann (2.29) no tendría sentido.

En un trabajo de Currie, Jordan y Sudarshan [59] se muestra que sí las partículas respetan la simetría de Poincaré y además satisfacen la condición de la línea de mundo, la cual establece que las trayectorias espacio-temporales de las partículas individuales se relacionan entre marcos de referencia siguiendo las transformaciones de Lorentz, entonces el Hamiltoniano del sistema es necesariamente el Hamiltoniano de un conjunto de partículas libres sin ningún término de interacción. No hay un Hamiltoniano que describa las interacciones entre las partículas relativistas. En principio puede pensarse que lo que prohíbe este teorema es la descripción Hamiltoniana de los sistemas relativistas de muchas partículas, entonces se podría desarrollar otro tipo de teorías que sean generalizaciones de las Hamiltonianas como teorías con constricciones [61], o bien teorías no Hamiltonianas [62], que puedan evitar alguna de las condiciones del teorema. Sin embargo, en [60] se demuestra el teorema con un formalismo Lagrangiano lo que implica que el resultado es más general.

Para que existan interacciones entre partículas relativistas se deben considerar como mediadas por un campo, es decir, aumentando los grados de libertad de tal forma que el teorema de no interacción ya no puede aplicarse y las trayectorias invariantes son descritas correctamente. Estas teorías son las llamadas de acción a distancia desarrolladas por Feynman y Wheeler [63] y las versiones estadísticas por Mangeney, Balescu y otros [64], [65] etc. Estas teorías además de describir al dinámica macroscópica del fluido, también describen la evolución del campo mediador, sin embargo son muy complejas y su principal dificultad es que, al tener un número infinito de grados de libertad, se tienen problemas de divergencias ${ }^{6}$. En trabajos recientes C. Tian [66] basado en el formalismo de acción a distancia encuentra una ecuación de Liouville manifiestamente covariante con un sólo tiempo, a partir de la cual puede obtenerse como un caso particular la ecuación de Boltzmann como la primera ecuación de una jerarquía infinita de ecuaciones, además de ecuaciones más generales y todas las correlaciones entre las distribuciones de más una partícula. El problema es la interpretación del parámetro temporal global que aparece en la ecuación de Liouville propuesta.

Otra forma de abordar el problema es considerando que un conjunto de $N$ partículas puede describirse también a través de sus trayectorias en el espacio-tiempo describiendo al sistema a través de un sólo punto en un espacio fase $8 N$ dimensional estático. El caso $N=1$ es el más sencillo ya que la componente

\footnotetext{
${ }^{6}$ Ver discusión en [10] y referencias ahí citadas.
} 
temporal de las coordenadas de la partícula puede identificarse con el parámetro de evolución, sin embargo, cuando $N>1$ esto no es posible ya que las componentes temporales de las coordenadas de las partículas están relacionadas entre ellas. El caso $N=1$ corresponde a lo descrito en las secciones anteriores, donde se utiliza la aproximación donde $N$ puntos en el espacio fase representan a las partículas que forman al gas, estas son libres en casi todo momento, excepto durante la colisión que es elástica y por tanto su única acción es modificar el 4-momento final de las partículas, después del cual siguen siendo libres ya que, aludiendo al caos molecular, no hay correlaciones entre las partículas.

Una aproximación que no entra en conflicto con el teorema de no-interacción es la que se describe en el apéndice $\mathrm{A}$, ahí se considera a la teoría cinética relativista como una aproximación a la teoría cuántica de campos. Una formulación de la teoría cuántica de campos es a través de la función de Wigner, esta función es la más cercana a una función de distribución a nivel cuántico, sin ser positiva definida. Pueden calcularse el tensor de energía momento y otras cantidades físicas como promedios pesados con la distribución de Wigner. De la ecuación de campo puede escribirse una ecuación dinámica para la función de Wigner. Al introducir el concepto de trazas parciales la ecuación para la función de Wigner puede escribirse como un sistema de ecuaciones acopladas, la aproximación consiste en utilizar únicamente la primera de estas ecuaciones cerrando el sistema con la hipótesis del caos molecular, el cual establece que no hay correlaciones iniciales entre las partículas. Con estas aproximaciones y reconociendo las amplitudes de dispersión y relacionándolas con la tasa de transición $W\left(p, p_{1} \mid p^{\prime}, p_{1}^{\prime}\right)$ de $(2.11)$, se demuestra que el resultado es precisamente la ecuación (2.12). A diferencia de la deducción heurística esta aproximación (ver apéndice A), puede evadir el resultado del teorema de no interacción ya que se está tratando con campos cuánticos y no partículas clásicas, la teoría cuántica de campo es el marco teórico donde pueden resolverse problemas como éste. 


\section{Análisis manifiestamente covariante de un gas simple relativista}

La función de distribución de velocidades del equilibrio para un gas la relativista, desempeña un papel clave en la descripción de varios efectos en la física de altas energías y en astrofísica por mencionar algunos. Recientemente, las simulaciones numéricas $[19,20,23]$ indican que la generalización relativista correcta de la distribución de Maxwell-Boltzmann en $d=1,2,3$ dimensiones espaciales, corresponde a la distribución de Jüttner. Dichas simulaciones son consistentes con una temperatura invariante relativista.

En este capítulo haremos ver que una temperatura invariante es consecuencia natural de la covarianza manifiesta de la teoría. Se presenta una nueva derivación manifiestamente covariante de la distribución de Jüttner y del teorema de equipartición. A diferencia de análisis previos, utilizamos coordenadas cartesianas en el espacio de momentos con $d+1$ dimensiones, $d$ de ellas las componentes espaciales. El uso del teorema de la multiplicación de funciones de Bessel se vuelve crucial para recuperar la forma conocida de la distribución de Jüttner [1]. Dado que los resultados de la teoría cinética en equilibrio deben estar de acuerdo con la termodinámica en el marco comóvil al gas, la norma del vector $\Theta^{\mu}$ que se introducirá más en la distribución se identificará con el recíproco de la temperatura en el marco comóvil.

Se discute como la elección de la normal a la hipersuperficie de integración, necesaria para definir cantidades termodinámicas, lleva a que estas tengan distintas propiedades de transformación. En particular se estudia el caso del momento relativista del gas y se argumenta que éste respeta las transformaciones de Lorentz siempre y cuando se utilice la velocidad del gas para definir dicha superficie. Esto resulta de hacer una analogía directa con el caso que resuelve la controversia Abraham-Lorentz del modelo clásico del electrón extendido, donde el momento del electrón parecía no tener el límite no relativista correcto.

Finalmente, al combinar los momentos estadísticos covariantes de la distribución de Jüttner, obtenemos una nueva forma del teorema de equipartición que también da cabida, tanto al vector térmico, como a la temperatura invariante comóvil y contiene, como un caso particular, una forma anterior no manifiestamente covariante. 


\subsection{Introducción}

La distribución de velocidades en el caso del gas relativista en equilibrio, se remonta a F. Jüttner, quien en 1911 obtuvo una distribución consistente para partículas relativistas que no considera la contribución de partículas con velocidades superiores a la velocidad de la luz en el vacío, denotada por $c$, y que sí figuran en la distribución de Maxwell. Esto se logró considerando la forma relativista de la energía de las partículas en un principio de máxima entropía, obteniendo la ahora llamada distribución de Jüttner [1].

$$
f=\frac{n}{4 \pi k T m^{2} c K_{2}\left(m c^{2} / k T\right)} e^{-\frac{\sqrt{\mathbf{p}^{2} c^{2}+m^{2} c^{4}}}{k T}}
$$

que aquí está escrita en el espacio de momentos. La masa en reposo de cualquiera de las partículas que forman el gas se denota por $m, k$ es la constante de Boltzmann y $K_{2}$ es una función de Bessel modificada de segundo orden [68]. Las cantidades restantes: la densidad de número de partículas $n$, la temperatura $T$ y las componentes espaciales del momento relativista $\mathbf{p}$, determinadas por un observador comóvil con el gas. Suponemos que se cumple la condición de capa de masa que relaciona la magnitud del momento espacial con la energía de la partícula, $(E / c)^{2}-\mathbf{p}^{2}=m^{2} c^{2}$. En el régimen no-relativista $|\mathbf{p}| \ll m c, k T \ll m c^{2}$, tenemos que $f \approx f_{\text {Maxwell }}$, donde $f_{\text {Maxwell }}$ es la distribución de velocidades de Maxwell. Así, las velocidades de las partículas más allá de $c$ son sólo un artificio de la aproximación no relativista Fig. (3.1).

La distribución de Jüttner en la forma (3.1) puede no ser muy conveniente, ya que no es manifiestamente covariante, es decir, no está escrita en términos de tensores de Lorentz que muestran explícitamente su comportamiento al investigar su descripción en distintos marcos en movimiento relativo. Para que sea manifiestamente covariante son necesarias dos piezas clave: la transformación bajo cambio de marco de referencia de la distribución $f$ y la de la temperatura $T$. Ambos han sido considerados en la literatura $[69,70]$ y $[6,12,71,72,73]$, para la temperatura y la función de distribución, respectivamente.

En el caso de la función de distribución primero es necesario considerar un gas contenido en una caja, para ello se multiplica (3.1) por la función característica $\chi$ que corresponde al recipiente contenedor del gas, tal que $\chi(\mathbf{x})=1$ para $\mathbf{x}$ dentro de la caja, y cero en caso contrario; la distribución resultante $\bar{f}(\mathbf{x}, \mathbf{p}):=\chi(\mathbf{x}) f(\mathbf{p})$ se define en el espacio fase de una partícula.

De esta forma un observador en el sistema de referencia donde el gas está en reposo, define $\bar{f} d^{3} x d^{3} p$ como el número de partículas del gas en un volumen de $d^{3} x$ ubicado en $\mathbf{x}$ y con momento $\mathbf{p}$ en el intervalo $d^{3} p$. Además $\bar{f}$ debe ser invariante de Lorentz [73, 71, 74]. Esto se ve fácilmente en el caso del equilibrio [74, 75]. (Para el caso fuera del equilibrio véase [71]): para un gas simple, el número de partículas $\mathcal{N}$ es invariante, por lo que $\mathcal{N}=\int d \mu \bar{f}$, con $d \mu=d^{3} x d^{3} p$, debe serlo también. Como $d \mu$ es una medida invariante de Lorentz debido a una compensación entre las transformaciones del momento espacial (por la relación de la capa de masa) y la medida espacial, $\bar{f}$ debe también ser invariante. Ahora bien, como $\chi$ es invariante ${ }^{1}$, entonces $f$ también lo es. Por lo tanto una forma manifiestamente covariante de $f$ equivale

\footnotetext{
${ }^{1}$ Consideremos dos marcos $S, S^{\prime}$, con el contenedor del gas situado en $S$. Con una velocidad relativa $v, \gamma=1 / \sqrt{1-v^{2} / c^{2}}$,
} 


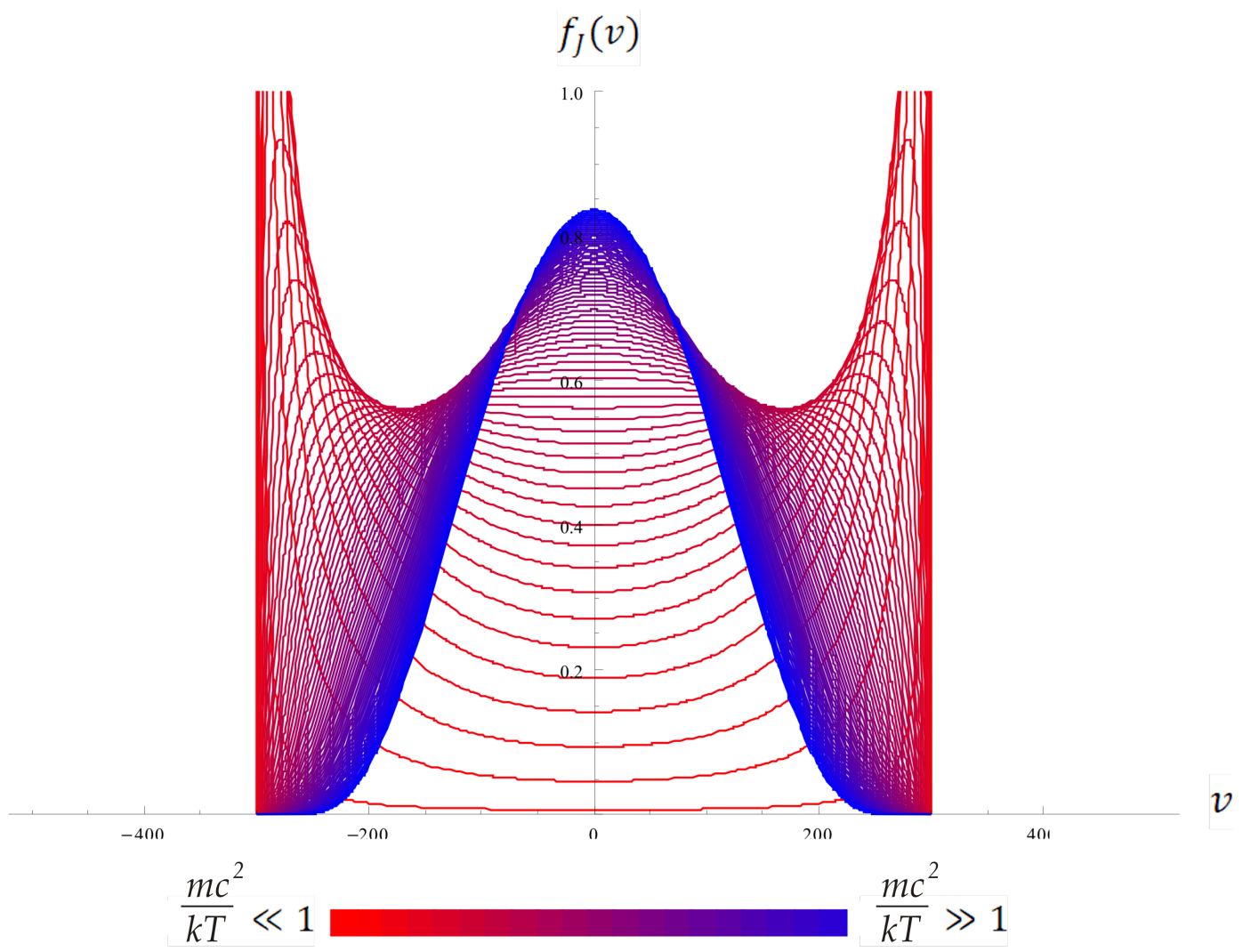

Figura 3.1: Función de Distribución de Jüttner. Se grafíca la distribución (3.1) en función de la velocidad para diferentes regímenes de temperatura. El color azul corresponde a $m c^{2} / k T \gg 1$, es decir, el caso no relativista, para este puede apreciarse que la distribución aproxima a la de Maxwell-Boltzmann. El color rojo es el caso ultra relativista $m c^{2} / k T \ll 1$, puede verse que conforme las partículas se acercan a la velocidad de la luz $c$, la distribución tiene dos picos en $c$ y $-c$.

a su invariancia manifiesta y, en particular, debe incluir el comportamiento de la temperatura bajo las transformaciones de Lorentz. La distribución de Jüttner invariante fue dada en [5, 6, 10, 72]. Se determinó mediante la introducción de coordenadas esféricas en la pseudoesfera asociados a la capa de masa en el espacio de cuatro momento relativista. Esto proporcionó un tratamiento elegante y fructífero que sin embargo no parece haber sido plenamente apreciado.

Recientemente se han propuesto alternativas a (3.1) donde la covarianza de Lorentz se incorpora de una manera diferente. En [14] Horwitz et al. presentan un enfoque mecánico cuántico que incluye un tiempo histórico invariante, éste fue considerado para obtener una ecuación de Boltzmann covariante con una solución estacionaria que lleva una relación entre la temperatura y el promedio de la energía diferente al usual en el caso ultra-relativista, mientras que en $[15,16]$ un principio de máxima entropía se combinó con un enfoque de teoría de grupo que involucra una cierta medida invariante, para obtener de nuevo

la contracción de Lorentz del volumen será $V^{\prime}=\gamma^{-1} V$ lo que lleva a que $\int d^{3} x^{\prime} \chi^{\prime}\left(x^{\prime}\right)=\int d^{3} x^{\prime} \chi(x), d^{3} x^{\prime}=\gamma^{-1} d^{3} x$, lo que demuestra la invariancia de $\chi$. 
una distribución de equilibrio modificada, la cual también se obtiene al considerar la no conservación de partículas [76, 22]. Ambas alternativas han sido recientemente criticadas por Debbasch [77].

Por otra parte simulaciones numéricas recientes usando la Dinámica Molecular para un gas relativista, en una dimensión y con dos componentes, desarrolladas por Cubero et al. [19], mostraron concordancia con la distribución de Jüttner (3.1). En este trabajo se consideran colisiones elásticas entre partículas con velocidades altas, de forma tal que en el algoritmo usual de Dinámica Molecular se reemplaza el momento no relativista después de la colisión por aquel que proviene de la conservación del 4-momento [19]. La temperatura en este trabajo fue definida a través de un teorema de equipartición, y demostró ser invariante bajo cambio de marcos de referencia [19]. El estudio del caso de dos dimensiones a lo largo de estas líneas ha sido desarrollada por Montakhab et al. [20] y Alino et al. [21], y para tres dimensiones espaciales en [23]. Para el caso de $d=3$ Peano et al. realizaron simulaciones adaptando al régimen relativista el método de Monte Carlo [22]. Sus resultados también indican que la distribución de Jüttner es la distribución de equilibrio correcta para el gas relativista. Sorprendentemente, en cuanto a la temperatura, este tipo de análisis nos lleva de nuevo a la discusión de muchos años sobre si un objeto en movimiento aparece más frio o más caliente $[69,70,19,20]$.

En este capítulo se obtiene la función de distribución de Jüttner manifiestamente invariante al utilizar coordenadas "cartesianas" en lugar de las coordenadas esféricas en el espacio de momento relativista en $(d+1)$-dimensiones [5,6]. Nuestro enfoque se puede considerar como una alternativa a derivaciones anteriores de la distribución relativista [12, 13], en el sentido de que evita la adopción de algún marco particular, que de lo contrario se plantearía la cuestión de la transformación de Lorentz de la temperatura. Nosotros únicamente aludimos a la estructura comóvil del gas al final del análisis al relacionar la descripción cinética con la termodinámica, en particular, para identificar la temperatura con la norma invariante de Lorentz de un vector térmico que ya había sido introducido antes $[5,6,10,72]$ y el cual está formado por el producto del inverso de la temperatura comóvil con la 4-velocidad del gas en su conjunto. Los casos de menores dimensiones estudiados recientemente [19, 21, 20, 22] están contenidos en nuestros resultados. Por lo tanto vemos que la temperatura comóvil juega un papel análogo a la masa en reposo de una partícula.

Esta interpretación se investiga en relación con el teorema de equipartición, ya que la existencia del vector térmico nos permite escribir una expresión manifiestamente covariante para este teorema. En trabajos previos como el de Tolman [78] o Landsberg [70], se considera una versión del teorema de equipartición que no es covariante manifiesta, obteniendo una expresión para la temperatura que no coincide con el promedio de la energía cinética de las partículas sino con otra cantidad. Las simulaciones numéricas antes mencionadas [19], [20], interpretan sus resultados con base en estos trabajos. La versión covariante del teorema de equipartición permitirá interpretar la temperatura comóvil como parte de la energía total del sistema. 
En nuestra discusión describimos el comportamiento de una distribución Planckiana cuando se hace uso de la temperatura invariante. También discutimos algunas de las dificultades para definir una temperatura no comóvil para un gas de partículas masivas, insistiendo en que la temperatura comóvil invariante parece ser la única posibilidad coherente para definir la temperatura de acuerdo a la teoría cinética relativista estándar.

La convención que seguiremos será la siguiente: Las componentes espacio-temporales de los tensores se indican mediante índices griegos: $\mu, \nu, \cdots=0,1,2,3$, la componente cero se refiere a la componente temporal, mientras que los componentes espaciales se caracterizan por los índices latinos $i, j, \cdots=1,2,3$. Se supone la convención de suma de Einstein para ambos tipos de índices en todas partes. La métrica de Minkowski tiene componentes $\eta_{\mu \nu}=\operatorname{diag}\{+,-,-,-\}$.

\subsection{Función de Distribución de Jüttner manifiestamente covariante}

Consideremos un gas simple compuesto por partículas idénticas de masa $m$ en un régimen relativista. Cada partícula tiene coordenadas de espacio-tiempo y cuatro-momento, dadas respectivamente por $x^{\mu}$ y $p^{\mu}$. La evolución de la función de distribución de una partícula $\bar{f}$ está dictada por la ecuación de Boltzmann relativista $[12,13,79]$

$$
p^{\mu} \frac{\partial \bar{f}}{\partial x^{\mu}}+m \frac{\partial \bar{f} \mathcal{K}^{\mu}}{\partial p^{\mu}}=\int\left(\bar{f}_{1}^{*} \bar{f}^{*}-\bar{f}_{1} \bar{f}\right) F \sigma d \Omega \frac{d^{3} p_{1}}{p_{1}^{0}}
$$

donde $\bar{f}_{1}^{*} \equiv \bar{f}\left(\mathbf{x}, \mathbf{p}_{1}^{*}, t\right)$, * indica una cantidad evaluada después de la colisión; $\sigma$ es la sección eficaz diferencial del proceso de dispersión, mientras que $\Omega$ es el ángulo sólido. $\mathcal{K}^{\mu}$ denota una 4-fuerza externa mientras que $F$ es el llamado flujo de invariante, $F=\sqrt{\left(p_{1}^{\mu} p_{\mu}\right)^{2}-m^{4} c^{4}}$, y $\frac{d^{3} p_{1}}{p_{1}^{0}}$ es la medida invariante en la capa de la masa. Estaremos interesados en el caso $\mathcal{K}^{\mu}=0$ correspondiente a no tener fuerzas externas. Al lado derecho de (3.2) se le conoce como integral de colisión.

Si se multiplican ambos lados de la ecuación (3.2) por una función arbitraria $\varphi(x, p)$ y se integra los momentos con la medida $\frac{d^{3} p}{p^{0}}$ se obtiene la llamada ecuación de transferencia

$$
\begin{aligned}
& \frac{\partial}{\partial x^{\mu}} \int \varphi p^{\mu} \bar{f} \frac{d^{3} p}{p^{0}}-\int \bar{f} \frac{d^{3} p}{p^{0}}\left[p^{\mu} \frac{\partial \varphi}{\partial x^{\mu}}+m \mathcal{K}^{\mu} \frac{\partial \varphi}{\partial p^{\mu}}\right]= \\
& \quad \frac{1}{4} \int\left(\varphi+\varphi_{1}-\varphi^{*}-\varphi_{1}^{*}\right)\left(\bar{f}_{1}^{*} \bar{f}^{*}-\bar{f}_{1} \bar{f}\right) F \sigma d \Omega \frac{d^{3} p_{1}}{p_{1}^{0}} \frac{d^{3} p}{p^{0}} .
\end{aligned}
$$

La ecuación (3.3) es la ecuación de balance para la cantidad física $\varphi$. En particular si utilizamos la función $\varphi=-k c \ln \left(\bar{f} h^{3}\right)+1$, con $h$ es una constante con dimensiones adecuadas para que el argumento del logaritmo quede sin dimensiones, obtenemos que:

$$
\frac{\partial S^{\mu}}{\partial x^{\mu}}=\varsigma
$$


donde se introdujo el 4-flujo de entropía

$$
S^{\mu}=-k c \int \frac{d^{3} p}{p^{0}} p^{\mu} \bar{f} \ln \left(\frac{h^{3} \bar{f}}{e}\right),
$$

y la tasa de producción de entropía

$$
\varsigma=\frac{k c}{4} \int \bar{f}_{1} \bar{f} \ln \left(\frac{\bar{f}^{*}{ }_{1} \bar{f}^{*}}{\bar{f}_{1} \bar{f}}\right)\left(\frac{\bar{f}^{*}{ }_{1} \bar{f}^{*}}{\bar{f}_{1} \bar{f}}-1\right) F \sigma d \Omega \frac{d^{3} p_{1}}{p_{1}^{0}} \frac{d^{3} p}{p^{0}} .
$$

Notemos que dado que las distribuciones son no negativas, si definimos $\mathrm{x}=\frac{f_{1}^{*} \bar{f}^{*}}{f_{1} \bar{f}}$, se cumple que para $\mathrm{x}=1$ el producto $(\mathrm{x}-1) \ln \mathrm{x}=0$, mientras que para $\mathrm{x} \neq 1 \mathrm{y} \mathrm{x}>0$, se tiene $(\mathrm{x}-1) \ln \mathrm{x}>0$. Esto nos dice que la producción de entropía nunca es negativa $\varsigma \geq 0$.

En particular el estado de equilibrio se encuentra cuando no hay producción de entropía [6, 12, 13, 72], es decir cuando $\bar{f}^{*} \bar{f}^{*}{ }_{1}=\bar{f} \bar{f}_{1}$, lo cual implica que la integral de colisión de (3.2) se anula. Tal condición se puede escribir como

$$
\ln f^{*}+\ln f_{1}^{*}=\ln f+\ln f_{1},
$$

donde la función característica $\chi$ que aparecía en $\bar{f}$ se cancela. La ecuación (3.7) se satisface por las cantidades que se mantienen invariantes durante la colisión ${ }^{2}$. En el caso relativista la ecuación (3.7) se satisface por las componentes del 4-momento; en [13] se prueba que los invariantes colisionales están dados por:

$$
\ln f=-\left(\Lambda(x)+\tilde{\Theta}_{\mu}(x) p^{\mu}\right) \Leftrightarrow f=\alpha(x) \exp \left(-\tilde{\Theta}_{\mu}(x) p^{\mu}\right) .
$$

El signo negativo en (3.8) se ha introducido por conveniencia, $p^{\mu}$ es el 4-momento de una partícula del gas, además $\alpha=e^{-\Lambda}$ y $\tilde{\Theta}_{\mu}$ son independientes de $p^{\mu}$. La función de distribución de equilibrio se obtiene completamente exigiendo que, al sustituir (3.8) en el lado izquierdo de (3.2) el resultado sea igual a cero. Esta sustitución deja $\Lambda$ independiente de $x^{\mu}$ y $\partial_{\nu} \tilde{\Theta}_{\mu}(x)+\partial_{\mu} \tilde{\Theta}_{\nu}(x)=0$, cuya solución es $\tilde{\Theta}_{\mu}(x)=\omega_{\mu \nu} x^{\nu}+\Theta_{\mu}$ y $\omega_{\mu \nu}=-\omega_{\nu \mu}$. Estos corresponden a los diez vectores de Killing $\left(6\right.$ de $\omega_{\mu \nu} x^{\nu}$ y 4 de $\Theta_{\mu}$ ) bajo los cuales la métrica espacio-temporal de Minkowski es invariante. Están asociados con las transformaciones de Lorentz y traslaciones espacio-temporales, respectivamente [80].

Como veremos en este capítulo el vector $\Theta_{\mu}$ se puede identificar con la 4-velocidad del fluido en su conjunto y por lo tanto, hereda las simetrías de espacio-tiempo en forma de movimientos rígidos: las líneas de mundo de los elementos del fluido vecinos mantendrán su separación siempre que se encuentren a lo largo de vectores de Killing [6]. Si restringimos tales movimientos a traslaciones, entonces sólo consideramos $\Theta_{\mu}$.

Para determinar $\alpha$ y $\Theta^{\mu}$ en (3.8) se supone que las cantidades físicas se relacionan con los momentos estadísticos de la distribución

$$
\mathcal{T}^{\mu_{1} \mu_{2} \ldots \mu_{n}}=c \int p^{\mu_{1}} p^{\mu_{2}} \ldots p^{\mu_{n}} \bar{f} \frac{d^{3} p}{p^{0}}
$$

\footnotetext{
${ }^{2}$ Nótese que esto se cumple para interacciones donde se conservan tanto las componentes del momento espacial como la energía, esto sucede en particular en las colisiones binarias elásticas.
} 
El primer momento corresponde al flujo de densidad de número de partículas $N^{\mu}$, mientras que el segundo es el tensor de energía-momento del gas, $T^{\mu \nu}$, respectivamente dadas por

$$
\begin{aligned}
N^{\mu} & =c \int p^{\mu} \bar{f} \frac{d^{3} p}{p^{0}}, \\
T^{\mu \nu} & =c \int p^{\mu} p^{\nu} \bar{f} \frac{d^{3} p}{p^{0}} .
\end{aligned}
$$

Si se introduce la siguiente integral invariante, conocida como funcional generadora [6]

$$
\mathcal{I} \equiv \int \mathrm{e}^{-\Theta_{\alpha} p^{\alpha}} \frac{d^{3} p}{p^{0}}
$$

los momentos de la distribución pueden escribirse como sus derivadas

$$
\begin{aligned}
\mathcal{T}^{\mu_{1} \mu_{2} \ldots \mu_{n}} & =(-1)^{n} \alpha \chi c \frac{\partial^{n} \mathcal{I}}{\partial \Theta_{\mu_{1}} \partial \Theta_{\mu_{2}} \ldots \partial \Theta_{\mu_{n}}}, \\
N^{\mu} & =-\alpha \chi c \frac{\partial \mathcal{I}}{\partial \Theta_{\mu}}, \\
T^{\mu \nu} & =\alpha \chi c \frac{\partial^{2} \mathcal{I}}{\partial \Theta_{\mu} \partial \Theta_{\nu}},
\end{aligned}
$$

Ahora sólo nos queda evaluar la funcional generadora $\mathcal{I}$. Para evaluar (3.12) se puede expresar la integral en coordenadas esféricas en la capa de masa $p^{0}=\sqrt{\mathbf{p}^{2}+m^{2} c^{2}}$, con lo que se tendrían las siguientes componentes para el 4-momento de la partícula $[5,6,72]$

$$
\begin{aligned}
p^{\mu}= & m c(\cosh \psi, \sinh \psi \sin \theta \cos \phi, \sinh \psi \sin \theta \sin \phi, \sinh \psi \cos \theta) \\
& 0 \leq \psi \leq \infty, 0 \leq \theta \leq \pi, 0 \leq \phi \leq 2 \pi
\end{aligned}
$$

en la pseudo-esfera $p^{\mu} p_{\mu}=m^{2} c^{2}$. De tal forma que (3.12) resulta

$$
\mathcal{I}=\int d \Omega^{(3)} d \psi \sinh ^{2} \psi \mathrm{e}^{-m c \Theta \cosh \psi}=4 \pi \frac{K_{1}(m c \Theta)}{m c \Theta}, \quad \Theta^{\mu} \Theta_{\mu}=\Theta^{2}
$$

donde $d \Omega^{(3)}$ es el elemento de ángulo solido en tres dimensiones espaciales, $K_{1}$ es la función de Bessel modificada de primer orden [68] y $\Theta^{\mu}$ se escoge a lo largo del eje $\psi=0$.

Para evaluar las componentes de los momentos estadísticos (3.14) y (3.15), se calculan las derivadas de (3.16) utilizando identidades de las funciones de Bessel

$$
\frac{d}{d u}\left[u^{-n} K_{n}(u)\right]=-u^{-n} K_{n+1}(u) .
$$

Los momentos así calculados se identifican con cantidades termodinámicas evaluadas en el marco comóvil, tal como la ecuación de estado del gas. Esto llevó a Israel [6] a identificar la norma del vector $\Theta^{\mu}$ con el inverso de la temperatura $T$ medida en el sistema comóvil al gas. 
Una forma diferente de calcular (3.12) es considerar que, al ser in invariante, se puede calcular en un marco práctico, por ejemplo uno en el que $\Theta^{\mu}=\left(\Theta^{0}, \boldsymbol{\Theta}=\mathbf{0}\right)$. Este caso se ha calculado en [12, 13]. Al darnos cuenta de que (i) $\Theta_{\mu}$ debe ser de tipo-tiempo ${ }^{3}$ para hacer converger la integral en (3.10) y que (ii) el único vector de tipo-tiempo disponible para el gas es su velocidad como un todo $\mathcal{U}^{\mu}$, se propone $\Theta^{\mu}=\frac{\mathfrak{u}^{\mu}}{k T}$, de modo que $T$ se identifica con la cantidad medida en el marco en el que $\mathcal{U}$, la parte espacial de $\mathcal{U}^{\mu}$, es igual a cero, es decir, se escoge el marco comóvil. Este enfoque en el que uno escoge el marco comóvil, plantea la pregunta acerca de cuál es la transformación de Lorentz de la temperatura.

Sería deseable poder combinar el enfoque 4-dimensional antes mencionado, con el más intuitivo y más fácil de manejar donde se eligen las componentes cartesianas de $\Theta^{\mu}$, de tal forma que sea posible investigar el comportamiento de la temperatura bajo transformaciones de Lorentz.

Debido a que nuestro análisis es el mismo independientemente del número de dimensiones espaciales, estudiaremos el caso de general en $d$ dimensiones espaciales. Por lo tanto, los índices tensoriales correrán de la siguiente manera: $\mu, \nu \cdots=0,1,2, \ldots, d$.

\subsubsection{Cálculo de $\alpha$}

Consideremos un marco general no comóvil con el gas de manera que las componentes espaciales de $\Theta^{\mu}$, es decir, $\boldsymbol{\Theta}$, son distintos de cero.

En un espacio $d$-dimensional la integral $\mathcal{I}$ dada por la ecuación (3.12), sólo requiere de cambiar la medida de integración de $d^{3} p$ a $d^{d} p$. Es posible adoptar coordenadas esféricas para la parte espacial del momento y escoger una dirección espacial particular de tal modo que el producto interno se escribe como $\boldsymbol{\Theta} \cdot \mathbf{p}=$ $|\Theta||\mathbf{p}| \cos \vartheta_{1}$.

En estas coordenadas tenemos que la medida de integración es $d^{d} p=|\mathbf{p}|^{d-1} d|\mathbf{p}| d \Omega^{(d)}$, donde $d \Omega^{(d)}=$ $\left(\sin \vartheta_{1}\right)^{d-2}\left(\sin \vartheta_{2}\right)^{d-3} \ldots\left(\sin \vartheta_{d-2}\right)^{1} d \vartheta_{1} d \vartheta_{2} \ldots d \vartheta_{d-2} d \varphi=\prod_{i=1}^{d-2}\left(\sin \vartheta_{i}\right)^{d-i-1} d \vartheta_{i} d \varphi$, y los ángulos varian de $0<\varphi<2 \pi$ y $0<\vartheta_{i}<\pi[81]$. De esta forma $\mathcal{I}$ puede escribirse como

$$
\mathcal{I}=S_{d-1} \int \frac{d|\mathbf{p}||\mathbf{p}|^{d-1}}{p^{0}}\left(\sin \vartheta_{1}\right)^{d-2} d \vartheta_{1} \mathrm{e}^{-\Theta_{0} p^{0}} \mathrm{e}^{|\Theta||\mathbf{p}| \cos \vartheta_{1}}
$$

Donde se identificó

$$
S_{d-1}=\frac{2 \pi^{\frac{d-1}{2}}}{\Gamma\left(\frac{d-1}{2}\right)},
$$

como la hiper-superficie de una esfera unitaria $(d-1)$-dimensional [81], que resulta de integrar sobre los ángulos $\varphi$ y $d \Omega^{(d-1)}$, excluyendo únicamente a $\vartheta_{1}$. Para integrar en $\vartheta_{1}$ es mejor utilizar la serie de la

\footnotetext{
${ }^{3}$ En Relatividad Especial los 4-vectores se clasifican según el signo de su norma. Así un vector tipo-tiempo es aquel cuya norma es mayor que uno, i.e. $A^{\mu} A_{\mu}>1$, un vector tipo-espacio tiene norma negativa $A^{\mu} A_{\mu}<1$ y también hay vectores de norma nula $A^{\mu} A_{\mu}=0$ a los cuales se les llama vectores nulos o tipo-luz. Ver por ejemplo [54].
} 
exponencial que contiene el producto de componentes espaciales

$$
\mathrm{e}^{|\boldsymbol{\Theta}||\mathbf{p}| \cos \vartheta_{1}}=\sum_{k=0}^{\infty} \frac{\left(|\boldsymbol{\Theta}||\mathbf{p}| \cos \vartheta_{1}\right)^{k}}{k !} .
$$

De esta forma (3.18) puede escribirse como

$$
\mathcal{I}=S_{d-1} \sum_{k=0}^{\infty} \frac{|\Theta|^{k}}{k !} \int_{0}^{\pi} \sin \vartheta_{1}^{d-2} \cos \vartheta_{1}^{k} d \vartheta_{1} \int \mathrm{e}^{-\Theta_{0} p^{0}} \frac{|\mathbf{p}|^{d-1+k}}{p^{0}} d|\mathbf{p}| .
$$

La integral angular restante en (3.18) es no trivial sólo para $k=2 n, n=0,1,2, \ldots$, esta integral está relacionada con las funciones Beta $B\left(\frac{2 n+1}{2}, \frac{d-1}{2}\right)$ [68], tal que

$$
\mathcal{I}=S_{d-1} \sum_{n=0}^{\infty} \frac{|\Theta|^{2 n}}{(2 n) !} B\left(\frac{2 n+1}{2}, \frac{d-1}{2}\right) \int \mathrm{e}^{-\Theta_{0} p^{0}}\left[p^{0^{2}}-m^{2} c^{2}\right]^{\frac{2 n+d-2}{2}} d p^{0}
$$

En (3.22) se hizo un cambio de la variable independiente de $|\mathbf{p}|$ por $p^{0}$ utilizando la relación de la capa de masa. Si ahora consideramos los cambios de variable $y^{4}=p^{0} / m c$ y $z_{0}=m c \Theta_{0}$, junto con la forma integral para las funciones de Bessel modificadas de primera especie dadas en [68]:

$$
\int_{1}^{\infty} \mathrm{e}^{-a x}\left(x^{2}-1\right)^{j-\frac{1}{2}} d x=\left(\frac{2}{a}\right)^{j} \frac{\Gamma\left(j+\frac{1}{2}\right)}{\Gamma\left(\frac{1}{2}\right)} K_{j}(a),
$$

la ecuación (3.22) toma la siguiente forma

$$
\begin{aligned}
\mathcal{I}= & S_{d-1} \sum_{n=0}^{\infty} \frac{|\Theta|^{2 n}}{(2 n) !} B\left(\frac{2 n+1}{2}, \frac{d-1}{2}\right)(m c)^{2 n+d-1}\left(\frac{2}{z_{0}}\right)^{\frac{2 n+d-1}{2}} \times \\
& \times \frac{\Gamma\left(n+\frac{d}{2}\right)}{\Gamma\left(\frac{1}{2}\right)} K_{\frac{2 n+d-1}{2}}\left(z_{0}\right) .
\end{aligned}
$$

En este punto, hacemos las siguientes consideraciones: (i) Utilizamos una expresión conocida de la función Beta en términos de las funciones Gamma [68], (ii) introducimos el vector $z^{\mu}=m c \Theta^{\mu}$, junto con $\beta \equiv$ $|\mathbf{z}| / z^{0}, 0 \leq \beta<1$, que se sigue del hecho de que $\Theta^{\mu}$ es un vector tipo-tiempo. De esta manera la ecuación (3.24) puede reescribirse como

$$
\mathcal{I}=2^{\frac{d+1}{2}}(m c)^{d-1}\left(\frac{\pi}{z_{0}}\right)^{\frac{d-1}{2}} \sum_{n=0}^{\infty} \beta^{2 n} z_{0}^{n} \frac{K_{n+\frac{d-1}{2}}\left(z_{0}\right)}{2^{n} n !} .
$$

La suma en (3.25) puede reducirse a una función de Bessel modificada al utilizar un teorema de multiplicación de las funciones de Bessel [82], a saber (C.20),

$$
K_{\nu}(\lambda x)=\lambda^{\nu} \sum_{l=0}^{\infty} \frac{(-1)^{l}\left(\lambda^{2}-1\right)^{l}\left(\frac{1}{2} x\right)^{l}}{l !} K_{\nu+l}(x),
$$

\footnotetext{
${ }^{4}$ Nótese que de la definición del 4-momento $y^{0}=\gamma$, el factor de Lorentz es la nueva variable de integración.
} 
con $\left|\lambda^{2}-1\right|<1$. Finalmente esto nos lleva a

$$
\mathcal{I}=2(m c)^{d-1}\left(2 \frac{\pi}{z}\right)^{\frac{d-1}{2}} K_{\frac{d-1}{2}}(z) .
$$

donde $z \equiv \sqrt{z^{\mu} z_{\mu}}$. La fórmula (3.26) es esencial para intercambiar la serie de funciones de Bessel en (3.25) por una función de Bessel modificada única en (3.27). La ecuación (3.27) se reduce a (3.16) cuando $d=3$. De (3.27) vemos que $\mathcal{I}$ es sólo función de un invariante $z=m c \Theta$. Ahora podemos obtener el 4-flujo de partículas relativistas a partir de (3.14)

$$
N^{\mu}=2 m^{d} c^{d+1} \alpha \chi\left(2 \frac{\pi}{z}\right)^{\frac{d-1}{2}} K_{\frac{d+1}{2}}(z) \frac{z^{\mu}}{z} .
$$

Podemos despejar el escalar $\alpha$ de la ecuación anterior

$$
\alpha \chi \equiv \frac{\sqrt{N^{\mu} N_{\mu}}}{2 c(m c)^{d} K_{\frac{d+1}{2}}(m c \Theta)}\left(\frac{m c \Theta}{2 \pi}\right)^{\frac{d-1}{2}} .
$$

De la ecuación (3.28) se puede deducir que $z^{\mu}$ y $N^{\mu}$ apuntan en la misma dirección. Notemos que $N^{\mu}=$ $N \mathcal{U}^{\mu} / c$, donde $N=\sqrt{N^{\mu} N_{\mu}}$; en particular en el marco comóvil $\mathcal{U}^{\mu} \rightarrow(c, \mathbf{0})$, y entonces $N=n c$, donde $n$ es la densidad de número de partículas en el marco comóvil. Esto implica que

$$
\Theta^{\mu}=\frac{\Theta}{c} U^{\mu}
$$

El significado físico de $\Theta$ dentro de este enfoque se analiza en la siguiente sección.

\subsection{2. $\quad \Theta^{\mu}$ y la Temperatura invariante}

Ahora buscamos identificar $\Theta^{\mu}$ con una cantidad termodinámica conocida. Tomemos como punto de partida la forma de Gibbs de la segunda ley de la termodinámica para un sistema cerrado [83, 72], que supondremos válida en el marco comóvil

$$
\delta U=T \delta \mathcal{S}-\mathcal{P} \delta V
$$

Debemos relacionar las cantidades estadísticas definidas antes, como el tensor de energía-momento (3.15) y el flujo de entropía (3.5) con la energía interna, $U$, la entropía, $\mathcal{S}$, la presión $\mathcal{P}$ y el volumen $\mathrm{V}$ que aparece en (3.31). Para esto vamos a introducir la función de distribución (3.8) y la ecuación (3.29), en la expresión para el tensor de energía-momento (3.15). Esto conduce a

$$
T^{\mu \nu}=-\frac{N}{\Theta}\left(\eta^{\mu \nu}-\frac{K_{\frac{d+3}{2}}(m c \Theta)}{K_{\frac{d+1}{2}}(m c \Theta)} \frac{\Theta^{\mu} \Theta^{\nu}}{\Theta} m c\right) .
$$

La presión comóvil se puede obtener a partir del tensor de energía-momento en $d$-dimensiones como

$$
\mathcal{P} \equiv-\frac{1}{d} \Delta_{\mu \nu} T^{\mu \nu}=\frac{N}{\Theta}
$$


donde el proyector ortogonal a la 4-velocidad hidrodinámica se define como $\Delta^{\mu \nu} \equiv \eta^{\mu \nu}-\mathcal{U}^{\mu} \mathcal{U}^{\nu} c^{-2}$. El flujo de la entropía $d$-dimensional (3.5) correspondiente se puede reescribir de forma conveniente como

$$
S^{\mu}=-k\left[\ln \left(\alpha h^{d}\right) N^{\mu}-T^{\mu \nu} \Theta_{\nu}\right] .
$$

Es importante mencionar que las cantidades $N^{\mu}, T^{\mu \nu}$ y $S^{\mu}$ son densidades, y por lo tanto no dependen del tamaño del sistema. Sin embargo, para incluir el hecho de que el gas está confinado dentro de una caja, es necesario utilizar la función característica $\chi(x)$ que se definió previamente en la introducción. En particular, integrando las cantidades antes mencionadas sobre $d$ dimensiones espaciales

$$
\begin{aligned}
\mathcal{N} & =c^{-1} \int_{\Sigma} N^{\mu} d \sigma_{\mu}, \\
\mathcal{S} & =c^{-1} \int_{\Sigma} S^{\mu} d \sigma_{\mu} . \\
\mathcal{G}^{\mu} & =c^{-1} \int_{\Sigma} T^{\mu \nu} d \sigma_{\nu},
\end{aligned}
$$

donde $d \sigma_{\mu}=n_{\mu} d^{d} x$, con $n_{\mu}$ un vector unitario normal a la hipersuperficie espacial $\Sigma$, y por lo tanto un vector de tipo-tiempo. Recordemos que la única dependencia en $x^{\mu}$ es a través de la función característica $\chi$, que está implícita en las definiciones de $S^{\mu}, N^{\mu}$ y $T^{\mu \nu}$, en las ecuaciones (3.5), (3.14) y (3.15), respectivamente.

Para calcular las integrales espaciales anteriores tomamos un hiperplano comóvil con el gas de manera que su normal unitaria esté dada por $n^{\mu}=\frac{\mathcal{U}^{\mu}}{c}=\frac{\Theta^{\mu}}{\Theta}$. Como la 4-velocidad hidrodinámica $\mathcal{U}^{\mu}$ es una propiedad intrínseca del sistema, esta elección ofrece una forma verdaderamente covariante de estas integrales [84]. Notemos que para una elección diferente como $n^{\mu}=\delta_{0}^{\mu}$, que corresponde al caso de un hiperplano a tiempo fijo, la integral que define al momento total del gas (3.37) daría un vector aparente en lugar de un verdadero vector de Lorentz ${ }^{5}$. Por lo tanto, el número de partículas $\mathcal{N}$ y la entropía $\mathcal{S}$ son evidentemente escalares, mientras que $\mathcal{G}^{\mu}$, el momento relativista del gas, es un vector de Lorentz. Integrando (3.34) junto con (3.35)-(3.37) conduce a,

$$
\mathcal{S}=-k\left[\mathcal{N} \ln \left(\alpha h^{d}\right)-\Theta_{\mu} \mathcal{G}^{\mu}\right]
$$

Ahora podemos hacer contacto con (3.31) evaluando todas las cantidades involucradas en el marco comóvil. Para evaluar la entropía (3.38) en el marco comóvil es necesario considerar (3.30) que, al multiplicarse por $\mathcal{G}^{\mu}$ nos lleva a $\Theta_{\mu} \mathcal{G}^{\mu} \rightarrow \Theta_{0} \mathcal{G}^{0}=\Theta_{\mathcal{G}^{0}}$. La diferencial de la entropía, (3.38), en el espacio termodinámico $(\Theta$ constante $)$ se convierte en

$$
\begin{aligned}
\delta \mathcal{S} & =k \Theta\left[\delta \mathcal{G}^{0}-\frac{\mathcal{N} \mathcal{P}}{c} \frac{\delta N}{N^{2}}\right], \\
& =\frac{k \Theta}{c}\left[\delta\left(c \mathcal{G}^{0}\right)+\mathcal{P} \delta V\right],
\end{aligned}
$$

\footnotetext{
${ }^{5}$ El carácter vectorial ante transformaciones de Lorentz del 4-momento del gas $\mathcal{G}^{\mu}$ se estudiará en la siguiente sección.
} 
donde se hizo uso de (3.29), (3.33) y la relación $\delta V=-\frac{\mathcal{N} \delta N}{N^{2}}$. La comparación de (3.31) con (3.39) da lugar a identificar la norma del vector $\Theta^{\mu}$

$$
\Theta=\frac{c}{k T},
$$

con la temperatura comóvil $T$. Para esto es necesario interpretar la cantidad $c \mathcal{G}^{0}$ como la energía interna relativista. Esta identificación es posible debido a que en el régimen de bajas velocidades se tiene que $c \mathcal{G}^{0} \approx \mathcal{N} m c^{2}+U_{\text {non-rel }}$, con $U_{\text {non-rel }}$ la energía interna usual del gas no relativista. Las expresiones (3.29), (3.30) y (3.40) completan el análisis de la función de distribución manifiestamente covariante que, expresada en términos de cantidades invariantes, $N, \Theta, m, c, \Theta^{\mu} p_{\mu}$, toma la forma

$$
f(p)=\frac{N}{2 c(m c)^{d} K_{\frac{d+1}{2}}(m c \Theta)}\left(\frac{m c \Theta}{2 \pi}\right)^{\frac{d-1}{2}} \exp \left(-\Theta_{\mu} p^{\mu}\right) .
$$

La ecuación (3.41) para $d=1,2$ y 3 se reduce a las funciones utilizadas en [19], [20, 21] y [23] respectivamente. La ecuación (3.1) se obtiene de (3.41) considerando $d=3$ y utilizando el marco comóvil al gas. Por otra parte (3.41) coincide con lo obtenido en [76] para dimensión $d$ arbitraria. La distribución relativista del equilibrio también se ha analizado últimamente desde una mecánica estadística relativista, utilizando el procedimiento de maximización de entropía [85] dando la misma distribución de Jüttner covariante.

Cabe destacar sin embargo, que en la deducción de (3.41) no se hicieron hipótesis a priori sobre la transformación de Lorentz de la temperatura. Esta se encontró al pedir que en el marco comóvil, se cumpliera la termodinámica del equilibrio.

\subsection{Momento total de un Gas relativista}

En un trabajo clásico de van Kampen [86], se establece que la energía y momento de un gas no transforman como un vector verdadero ante transformaciones de Lorentz debido a que el gas, al estar confinado en algún recipiente, no es un sistema cerrado ya que interactúa con las paredes de dicho contenedor. Este problema se ha retomado recientemente por Dunkel et al. en [23], haciendo notar que las propiedades de transformación del momento total dependen de la superficie de integración que se elige en (3.37).

Para comprender esta afirmación, uno puede preguntarse sobre el comportamiento de $\mathcal{G}^{\mu}$ bajo transformaciones de Lorentz. Para ello, una posibilidad es seguir la misma línea de razonamiento que en el caso del modelo de electrón clásico extendido dado por Rohrlich [84], [87]. Este argumento aclaró la controversia de Abraham-Lorentz sobre el momento relativista del electrón clásico.

Con base en el análisis de [87], el momento total $\mathcal{P}_{\text {total }}^{\mu}$ del sistema compuesto por el gas y la caja que lo contiene, es un vector de Lorentz conservado que se puede descomponer como la suma de vectores de Lorentz verdaderos o aparentes

$$
\mathcal{P}_{\text {total }}^{\mu}=\mathcal{G}^{\mu}+\Pi^{\mu}=\mathcal{G}^{(\mu)}+\Pi^{(\mu)}
$$


donde $(\mu)$ indica que tales objetos son vectores aparentes, y $\Pi^{\mu}$ es la contribución de la caja. Un vector es verdadero si se comporta adecuadamente ante transformaciones de Lorentz; un vector aparente no respeta las transformaciones de Lorentz. Como en el caso del modelo clásico del electrón, la diferencia entre verdadero y aparente del 4-momento se establece tal que en el límite no relativista, las componentes de $\mathcal{G}^{\mu}$ coinciden exactamente con la energía y el impulso no relativistas del gas, mientras que las componentes $\operatorname{de} \mathcal{G}^{(\mu)}$ no, [87].

Los conceptos de vector verdadero o aparente, están relacionados con la hipersuperficie que se elige al integrar el tensor de energía-momento $T^{\mu \nu}$ en (3.37). Cuando se utiliza una hipersuperficie cuya normal unitaria es $\frac{\mathcal{U}^{\mu}}{c}$, donde $\mathcal{U}^{\mu}$ es la velocidad hidrodinámica, es decir, se utiliza un marco comóvil con el gas, tenemos vectores de Lorentz verdaderos; cuando se utiliza una hipersuperficie que se caracteriza con una normal arbitraria a tiempo fijo $\delta_{0}^{\mu}$, tendremos vectores aparentes.

En el caso de vectores de Lorentz verdaderos, el recipiente que contiene al gas no es necesario para garantizar el carácter de vector verdadero de $\mathcal{P}_{\text {total }}^{\mu}$, aunque es necesario para explicar el equilibrio del sistema. Por esta razón nosotros elegimos utilizar la hipersuperficie cuya normal apunta en la misma dirección de la velocidad hidrodinámica $n^{\mu}=\frac{u^{\mu}}{c}=\frac{\Theta^{\mu}}{\Theta}$. Entonces sustituimos esta elección en la definición del momento total del gas (3.37), utilizando la expresión del tensor de energía-momento hallada antes (3.32). De esta manera obtenemos:

$$
\mathcal{G}^{\mu}=\mathcal{N} \frac{\Theta^{\mu}}{\Theta^{2}}\left[m c \Theta \frac{K_{\frac{d+3}{2}}(m c \Theta)}{K_{\frac{d+1}{2}}(m c \Theta)}-1\right] .
$$

Por otro lado si utilizáramos vectores aparentes sólo la suma de las dos contribuciones del gas y del contenedor forman un vector verdadero (3.42), por esta razón en este caso la contribución de la caja es esencial. Podemos interpretar lo establecido en [23] y [86], sobre la forma en cómo transforman la energía y el momento, diciendo que ahí se utilizaron vectores aparentes en lugar de vectores de Lorentz verdaderos. Con la elección de la normal $n^{\mu}=\delta_{0}^{\mu}$, aunque $\mathcal{N}$ y $\mathcal{S}$ no se alteran, la integral (3.37) del momento resulta un vector aparente que toma la siguiente forma

$$
\mathcal{G}^{(\mu)}=\frac{\mathcal{N}}{\Theta}\left[m c \frac{K_{\frac{d+3}{2}}(m c \Theta)}{K_{\frac{d+1}{2}}(m c \Theta)} \frac{\Theta^{0}}{\Theta} \Theta^{\mu}-\eta^{\mu 0}\right] .
$$

Es importante notar que (3.43) y (3.44) coinciden únicamente en el marco comóvil $\mathfrak{U}=0$.

Ahora bien, según el argumento de Dunkel et al. en [23], el hecho de tener vectores aparentes se debe a que el gas no es un sistema cerrado sino que interactúa con las paredes del contenedor, por lo tanto el tensor de energía-momento no puede ser una cantidad conservada $\partial_{\mu} T^{\mu \nu} \neq 0$. No se hace referencia a la elección de la normal a la hipersuperficie.

De acuerdo a la argumentación usual [75], si $T^{\mu \nu}$ se conserva sus integrales espaciales son independientes de la hipersuperficie espacial $\Sigma$ que se elija para dicha integración debido al teorema de Gauss:

$$
0=\int_{\Omega} \partial_{\mu} T^{\mu \nu} d^{4} x=\oint_{\partial \Omega} T^{\mu \nu} d \sigma_{\mu}
$$




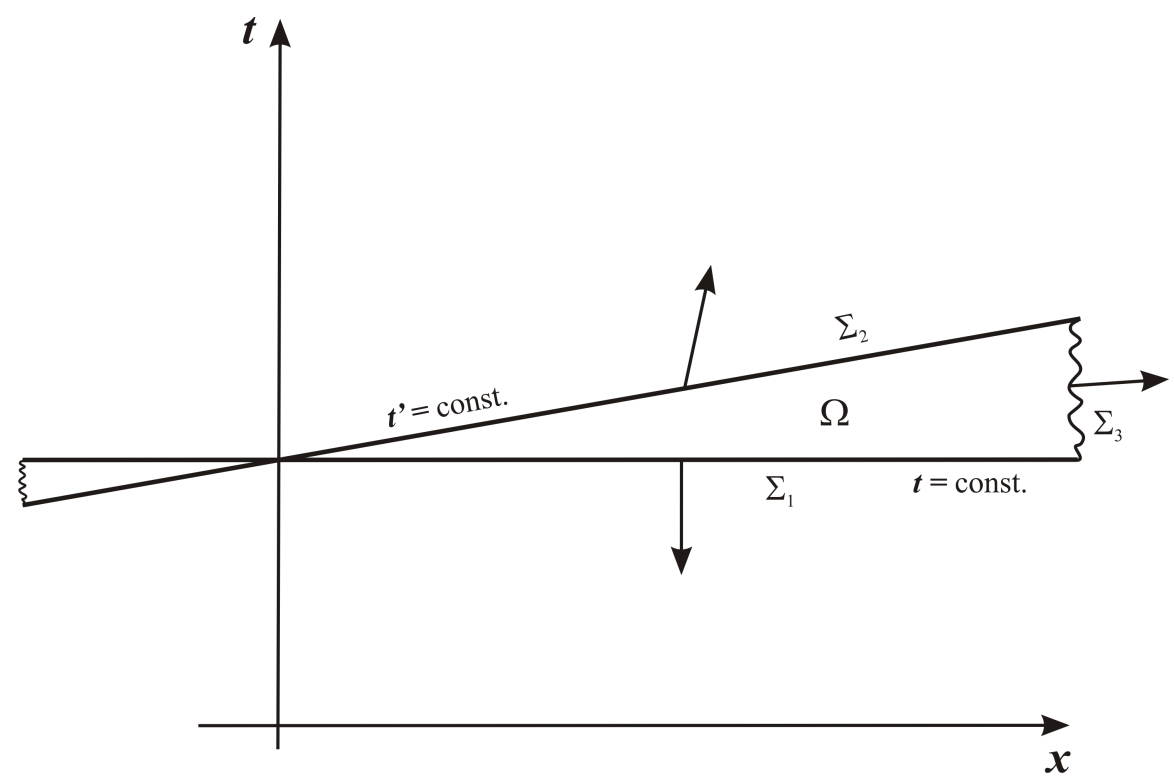

Figura 3.2: Aplicación del teorema de Gauss en la región $\Omega$ cuya frontera orientada está formada por hipersuperficies correspondientes a distintos observadores inerciales $\partial \Omega=\Sigma_{1}+\Sigma_{2}+\Sigma_{3}$.

donde $\Omega$ es una región espacio-temporal cerrada con $\partial \Omega$ una frontera orientable. Si la frontera se descompone como en Fig. 3.2, entonces $\partial \Omega=\Sigma_{1}+\Sigma_{2}+\Sigma_{3}$, donde $\Sigma_{2}$ tiene una normal que apunta en la dirección opuesta a la normal de $\Sigma_{1}$ y $\Sigma_{3}$ es una superficie en infinito que no contribuye a (3.45). Por consiguiente

$$
\int_{\Sigma_{1}} T^{\mu \nu} d \sigma_{\mu}^{(1)}=-\int_{\Sigma_{2}} T^{\mu \nu} d \sigma_{\mu}^{(2)}
$$

que implica que el momento del gas es independiente de la hipersuperficie de integración. Si $T^{\mu \nu}$ no se conserva, las integrales espaciales correspondientes evidentemente dependerán de la hipersuperficie que se elija como superficie de integración. La expresión (3.46) nos dice que el momento del gas estará relacionado por una transformación de Lorentz visto por observadores en los marcos de referencia correspondientes a las hipersuperficies $\Sigma_{1}$ y $\Sigma_{2}$

$$
\mathcal{G}^{\nu}=\Lambda_{\nu^{\prime}}^{\nu} \mathcal{G}^{\nu^{\prime}}
$$

donde $\Lambda_{\nu^{\prime}}^{\nu}$ es la matrix asociada a las transformaciones de Lorentz:

$$
\left[\Lambda_{\nu^{\prime}}^{\nu}\right] \rightarrow\left(\begin{array}{cccc}
\gamma & -\beta \gamma & 0 & 0 \\
-\beta \gamma & \gamma & 0 & 0 \\
0 & 0 & 1 & 0 \\
0 & 0 & 0 & 1
\end{array}\right)
$$

En este argumento no se ha hecho referencia a la elección de la normal a la superficie de integración ¿Cómo interpretamos entonces (3.46) a la luz de las distintas posibilidades de elegir la normal a la hipersuperficie? La elección de $n^{\mu}=\delta_{0}^{\mu}$ implica un proceso de medición en superficies con distinta simultaneidad. Imaginemos que $T^{\mu \nu}$ se conserva, $\partial_{\mu} T^{\mu \nu}=0$. Nos preguntamos qué forma debe tener este tensor tal que (3.46) 
involucre un proceso de medición simultánea en cada marco, es decir si $d \sigma^{\mu(1)}=\delta_{0}^{\mu} d V$ y $d \sigma^{\mu(2)}=-\delta_{0^{\prime}}^{\mu^{\prime}} d V^{\prime}$, donde $d V$ y $d V^{\prime}$ son los elementos de volumen espacial medidos en los marcos de referencia correspondientes a las hipersuperficies $\Sigma_{1}$ y $\Sigma_{2}$ respectivamente, es decir, los integrandos se escribirían

$$
T^{0 \nu} d V=\Lambda_{\nu^{\prime}}^{\nu} T^{0^{\prime} \nu^{\prime}} d V^{\prime}
$$

Al involucrar un proceso de medición se requiere que (3.49) sea compatible con la contracción de Lorentz del volumen $d V^{\prime}=\gamma^{-1} d V$, lo cual implica que el tensor $T^{\mu \nu}$ debe cumplir que

$$
\gamma T^{0 \nu}=\Lambda_{\nu}^{\nu} T^{0^{\prime} \nu^{\prime}}
$$

es claro que la ecuación (3.50) no se cumple para cualquier tensor, en realidad impone restricciones sobre la forma de $T^{\mu \nu}$ para que éste sea compatible con mediciones y con las trasformaciones de Lorentz. La forma que tomaría este tensor, suponiendo isotropía espacial es

$$
T^{\mu \nu}=T \mathcal{U}^{\mu} \mathfrak{U}^{\nu}, \quad \text { con } T=T^{00} \quad \text { en el marco comóvil. }
$$

Éste corresponde al tensor de energía-momento de un polvo. En esta interpretación, el teorema de Gauss no sólo implica cierta dependencia en las coordenadas espacio-temporales, sino también la forma que toma el tensor si queremos que sea compatible con mediciones en marcos con diferente simultaneidad. Así para estudiar un gas con un tensor de energía-momento distinto, tendríamos que imponer $\partial_{\mu} T^{\mu \nu}=F^{\nu}$, con $F^{\nu}$ la contribución de las paredes que introduce factores proporcionales a la presión, de tal forma que al integrarlo cancelará términos en (3.44) para que el momento total (3.42) tome una forma compatible con (3.51), [88]. Esta es la interpretación seguida por Dunkel.

Sin embargo, recordemos que se eligió desde el comienzo utilizar como normal aquella que nos da vectores aparentes que no están conectados por una transformación de Lorentz; por lo tanto parece que no es posible sostener el argumento van Kampen y Dunkel, ya que no es el teorema de Gauss lo que define el carácter vectorial sino la elección de la normal.

Por otro lado, podemos preguntarnos sobre la divergencia del tensor de energía-momento (3.32). Recordemos que para calcular $N$ es necesario considerar la función característica $\chi(x)$ que implica que el gas está únicamente en cierta región del espacio $V$. En general está función puede escribirse como

$$
\chi(x)= \begin{cases}1, & x \in V \\ 0, & x \notin V\end{cases}
$$

Para realizar el cálculo de la divergencia consideremos un gas en una dimensión espacial $d=1$, en este caso, como se ve en [19], la función característica puede escribirse para una caja de tamaño $L$ como

$$
\chi_{1 D}(x)=H(x) H(L-x),
$$

donde $H(x)$ es la función escalón de Heaviside definida como

$$
H(x)= \begin{cases}1, & x \geq 0 \\ 0, & x<1\end{cases}
$$


La única dependencia en $x$ del tensor de energía-momento entra a través de la función característica, por tanto la divergencia de $T^{\mu \nu}$ será proporcional a la divergencia de $\chi$, que en $d=1$ es:

$$
\frac{d}{d x} T^{\mu \nu} \propto \frac{d}{d x} \chi_{1 D}(x)=\delta(x) H(L-x)-\delta(L-x) H(x),
$$

donde claramente podemos notar que la derivada de la función característica es distinta de cero. Sin embargo, del lado derecho aparecen distribuciones, por lo tanto el valor particular de la derivada en (3.55) tiene sentido como funciones generalizadas, es decir, hasta realizar una integración:

$$
\int \frac{d}{d x} T^{\mu \nu} d x \propto \int[\delta(x) H(L-x)-\delta(L-x) H(x)] d x=0
$$

Es interesante hacer notar que el lado izquierdo de (3.56) es precisamente el lado izquierdo de la ecuación (3.45), es decir, la aplicación del teorema de Gauss para un gas en una caja $d=1$ implica que el momento total del gas no depende de la superficie de integración que se elija.

Esto no concuerda con la elección de $n^{\mu}=\delta_{0}^{\mu}$, como normal a la hipersuperficie. Una elección de la normal a la hipersuperficie de integración compatible con (3.56), es utilizar una propiedad intrínseca del sistema, es decir, a la velocidad hidrodinámica $n^{\mu}=\mathcal{U}^{\mu} / c$, Fig. 3.3. De esta forma no hay ambigüedad en las propiedades de transformación en (3.46) y (3.47) y la interpretación es directa, el momento del gas es un vector verdadero, por lo tanto, está relacionado a través de una transformación de Lorentz para distintos observadores inerciales. Además, es fácil ver que este resultado es válido para cajas cúbicas en cualquier dimensión espacial.

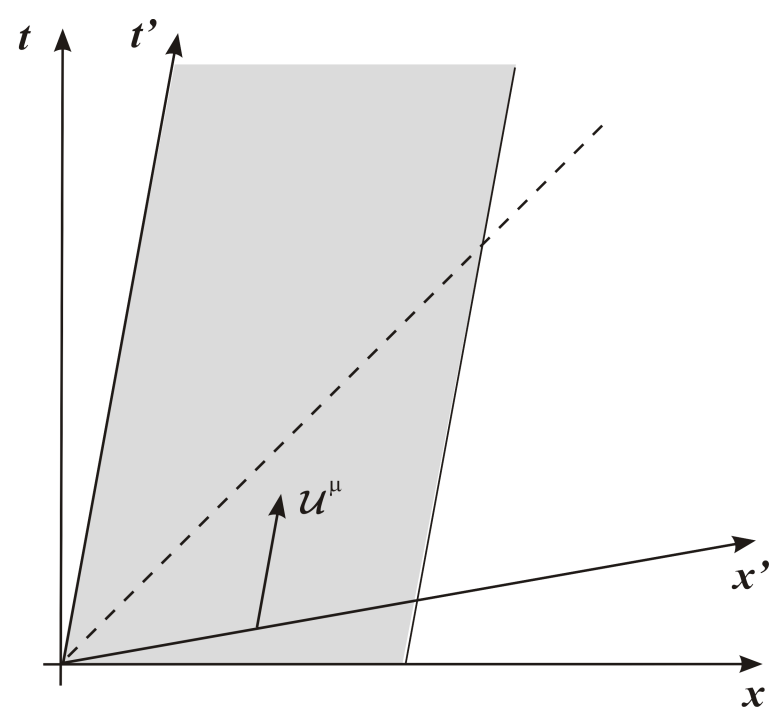

Figura 3.3: Elección de $n^{\mu}=\mathcal{U}^{\mu} / c$ como normal a la hipersuperficie espacial comóvil al gas.

El uso de una característica intrínseca al sistema como $\mathcal{U}^{\mu} / c$, para definir la normal a la hipersuperficie de integración puede apreciarse con la siguiente analogía sencilla. Supongamos una partícula cargada en un campo externo. Si nuestro sistema es únicamente la partícula, una translación de la partícula modificará su 
interacción con el campo. A esta acción se le llama traslación activa, es cambiar el sistema de referencia dinámicamente. Sin embargo, si consideramos al sistema como las partículas y las fuentes del campo, entonces una translación llevará a ambos, partículas y las fuentes a un nuevo marco de referencia y las interacciones no cambiará. A esto se conoce como transformación pasiva, se deja el sistema sin modificar, pero viéndolo desde otro marco de referencia. Al elegir como normal a la hipersuperficie a la velocidad hidrodinámica, no estamos tomando ningún elemento externo para describirlo, por lo tanto, las variables termodinámicas no tendrán ningún problema con las transformaciones activas y pasivas.

Finalmente queremos comentar sobre la solución propuesta por Dunkel et al., [23]. Ellos escogieron para realizar las integrales sobre la hipersuperficie invariante del cono de luz pasado de un evento $\mathfrak{E}$ con coordenadas espacio-temporales $\left(\xi^{0}, \xi^{i}\right)$. En esta hipersuperficie el impulso del gas, según lo calculado en [23], sería

$$
\mathcal{G}_{L C}^{\mu}=\frac{\mathcal{N}}{\Theta}\left[m c \frac{K_{\frac{d+3}{2}}(m c \Theta)}{K_{\frac{d+1}{2}}(m c \Theta)}\left(\frac{\Theta^{0}}{\Theta}-\frac{\Theta_{i} \xi^{i}}{\Theta \xi}\right) \Theta^{\mu}-\left(\eta^{\mu 0}-\frac{\eta^{\mu i} \xi_{i}}{\xi}\right)\right]
$$

Aunque, parece ser una manera accesible de definir experimentalmente magnitudes termodinámicas medibles [23], esta propuesta depende de las coordenadas de espacio-tiempo del evento $\mathfrak{E}$. Si el evento está en el origen del marco comóvil del gas (o de un observador relativo), entonces (3.57) se reduce a (3.44). Anticipamos esta dificultad debido a que se escogió un evento externo para definir las cantidades físicas para el sistema.

El carácter de vector aparente está relacionado con la realización de mediciones en dos marcos de referencia con distinta simultaneidad. Esto lleva a que las cantidades resultantes no respeten las transformaciones de Lorentz, o bien, si se exige que se respeten, restringe la forma de las componentes que puede tomar la cantidad tensorial involucrada. Utilizando cantidades intrínsecas al sistema se pueden formar cantidades que sigan la transformaciones de Lorentz al realizar cambios entre marcos y observadores inerciales [88].

\subsection{Teorema de equipartición relativista}

El Teorema de equipartición de la energía, es un resultado no relativista, que relaciona a la temperatura con el promedio de la energía cinética de las partículas que conforman el gas. Los primeros trabajos donde se extendió este resultado al ámbito relativista son aquellos de Tolman y Landsberg [78, 70] y una primera observación que se extrae de ellos es que no puede darse la misma interpretación a la temperatura como promedio de la energía, como sucede en el caso no relativista.

Como hemos demostrado en la sección anterior el enfoque manifiestamente covariante relativista de la función de distribución de equilibrio revela la conveniencia de utilizar una temperatura invariante asociada con el marco comóvil del gas. Por lo tanto, resulta interesante investigar el papel que juega la temperatura invariante dentro de una formulación manifiestamente covariante del teorema de equipartición relativista. 
En la literatura hay versiones del teorema de equipartición relativista que no están escritas en un lenguaje manifiestamente covariante, estas fueron previamente examinadas por Tolman [78] y más tarde por otros [89]. Estas versiones del teorema de equipartición se han utilizado como un criterio para determinar la transformación de Lorentz de la temperatura, sin embargo, estos intentos ya habían sido criticados por Landsberg [70] quien hace énfasis en que se pueden acomodar tanto la temperatura invariante, como una temperatura que transforme ante un cambio de marco de referencia, una temperatura móvil. Recientemente Cubero et al. [19] realizaron simulaciones numéricas que indican la existencia de una temperatura invariante sobre la base del teorema de equipartición relativista de [70], refiriéndose entonces a la temperatura invariante incluida en el análisis de Landsberg, pero dejando abierta la posibilidad de la existencia de una temperatura móvil. En esta sección daremos una forma manifiestamente covariante del teorema de equipartición que no sólo contiene una temperatura invariante, sino que incluye la versión de Landsberg de dicho teorema. Por otra parte en nuestra versión, el teorema es claramente expresado en términos del momento total del gas, de donde puede leerse que hay una parte cinética del momento total que es la contribución relevante al teorema de equipartición.

Comencemos por reexpresar la funcional generadora $\mathcal{I}(3.12)$ en el espacio de energía y momento en $(d+1)$-dimensiones [12]

$$
\mathcal{I}=2 \int e^{-\Theta_{\mu} p^{\mu}} \delta\left(p_{\sigma} p^{\sigma}-m^{2} c^{2}\right) H\left(p^{0}\right) d^{d+1} p
$$

donde $H\left(p^{0}\right)$ es la función escalón unitario de Heaviside que restringe (3.58) a las energías positivas. La condición de capa de masa se toma en cuenta a través de la distribución delta de Dirac.

Lo siguiente es hacer una extensión covariante del argumento original de Tolman [78], que consideraba únicamente la densidad de partículas, aquí hacemos esta extensión debido a que las derivadas de la funcional $\mathcal{I}$ se relacionan con cantidades físicas, en particular la densidad de energía es la componente temporal de su primera derivada. Lo siguiente es integrar por partes $d+1$ veces, una por cada $p^{\mu}$. Se descartan los términos de frontera en infinito y obtenemos

$$
\mathcal{I}=-\frac{2}{d+1} \int p^{\nu} \frac{\partial}{\partial p^{\nu}}\left[e^{-\Theta_{\mu} p^{\mu}} \delta\left(p_{\sigma} p^{\sigma}-m^{2} c^{2}\right)\right] H\left(p^{0}\right) d^{d+1} p
$$

Para integrar sobre $p^{0}$ aplicamos las propiedades de la delta de Dirac, que finalmente nos lleva a

$$
\mathcal{I}=\frac{1}{d} \int e^{-\Theta_{\mu} p^{\mu}}\left[\left(\frac{|\mathbf{p}|}{p^{0}}\right)^{2}+\Theta_{\mu} \frac{\partial p^{\mu}}{\partial p^{i}} p^{i}\right] \frac{d^{d} p}{p^{0}} .
$$

Sorprendentemente, y a pesar del segundo término en (3.60) contiene los componentes espaciales $p_{i},(3.60)$ es una cantidad invariante de Lorentz. 
Para conectar (3.60) con las cantidades físicas se inserta $\mathcal{I}$ en (3.14) y (3.15) para obtener

$$
\begin{aligned}
N^{\alpha} & =\frac{c}{d} \int \frac{d^{d} p}{p^{0}} f\left\{p^{\alpha}\left[\left(\frac{|\mathbf{p}|}{p^{0}}\right)^{2}+p^{i} \frac{\partial p^{\mu}}{\partial p^{i}} \Theta_{\mu}\right]-p^{i} \frac{\partial p^{\alpha}}{\partial p^{i}}\right\}, \\
T^{\alpha \beta} & =\frac{c}{d} \int \frac{d^{d} p}{p^{0}} f\left\{p^{\alpha} p^{\beta}\left[\left(\frac{|\mathbf{p}|}{p^{0}}\right)^{2}+p^{i} \frac{\partial p^{\mu}}{\partial p^{i}} \Theta_{\mu}\right]-p^{i} \frac{\partial p^{\alpha} p^{\beta}}{\partial p^{i}}\right\} .
\end{aligned}
$$

Integrando espacialmente de nuevo de (3.61) y (3.62) de acuerdo con 3.35) y (3.37) tenemos

$$
\begin{aligned}
d & =\Theta_{\mu}\left\langle\left\langle p^{i} \frac{\partial p^{\mu}}{\partial p^{i}}\right\rangle\right\rangle \\
\mathcal{G}^{\alpha} & =\frac{\mathcal{N}}{d}\left[\Theta_{\mu}\left\langle\left\langle p^{i} \frac{\partial p^{\mu}}{\partial p^{i}} p^{\alpha}\right\rangle\right\rangle-\left\langle\left\langle p^{i} \frac{\partial p^{\alpha}}{\partial p^{i}}\right\rangle\right\rangle\right] .
\end{aligned}
$$

donde se utilizó $\langle\langle\cdot\rangle\rangle \equiv \frac{1}{\mathcal{N}} \int \cdot f \chi d^{d} x d^{d} p$. Cabe mencionar que la ecuación (3.63) es sólo la forma manifiestamente covariante del teorema de equipartición correspondiente a la propuesta por Tolman y Landsberg [78, 70], respectivamente, expresada utilizando la temperatura invariante comóvil $T=c / k \Theta$, ec. (3.40). Con respecto a la covarianza (3.63), podemos notar que a pesar de que contiene la suma espacial $p^{i} \frac{\partial}{\partial p^{i}}$, se llega a partir de la ecuación manifiestamente covariante (3.58). Esto es análogo a reconocer la invariancia de $\frac{d^{3} \mathbf{p}}{p^{0}}$. Es evidente que el argumento es válido también para los dos términos en el lado derecho de la ecuación (3.64).

Cuando observamos que ambas ecuaciones (3.63) y (3.64) comparten un término común, esto sugiere la forma como podría insertarse el momento relativista en una expresión del teorema de equipartición covariante. Para proseguir necesitamos determinar el primer término en (3.64), sin embargo, esto lo podemos hacer comparando con la forma explícita el momento del gas calculada en (3.43). Entonces, al igualar la proyección $\Theta_{\alpha} \mathcal{G}^{\alpha}$ de (3.64) y (3.43) podemos identificar

$$
\frac{\Theta_{\alpha} \Theta_{\mu}}{d}\left\langle\left\langle p^{i} \frac{\partial p^{\mu}}{\partial p^{i}} p^{\alpha}\right\rangle\right\rangle=\frac{K_{\frac{d+3}{2}}(m c \Theta)}{K_{\frac{d+1}{2}}(m c \Theta)} m c \Theta,
$$

de donde finalmente llegamos a la siguiente expresión

$$
\begin{aligned}
\frac{\Theta_{\mu} \mathcal{G}^{\mu}}{\mathcal{N}}-z & =\mathcal{F}_{d}(z), \\
\mathcal{F}_{d}(z) & \equiv z \frac{K_{\frac{d+3}{2}}(z)}{K_{\frac{d+1}{2}}(z)}-1-z .
\end{aligned}
$$

Es interesante notar que, en la misma forma que la energía de una partícula con momento $p_{\text {part }}^{\mu}$ determinado por un observador con velocidad $U_{\text {obs }}^{\nu}$ está dado por $E_{\text {obs }}=p_{\nu \text { part }} U_{\text {obs }}^{\nu}$, podemos interpretar $\frac{1}{\Theta} \Theta_{\mu} \mathcal{G}^{\mu}$ como la energía del gas determinada por el observador comóvil.

$\mathcal{F}_{d}(z)$ se reduce a los valores usuales del teorema de equipartición en los casos límite (ver fig. 3.4),

$$
\mathcal{F}_{d}(z)= \begin{cases}\frac{d}{2}, & z \gg 1 \\ d, & z \ll 1\end{cases}
$$




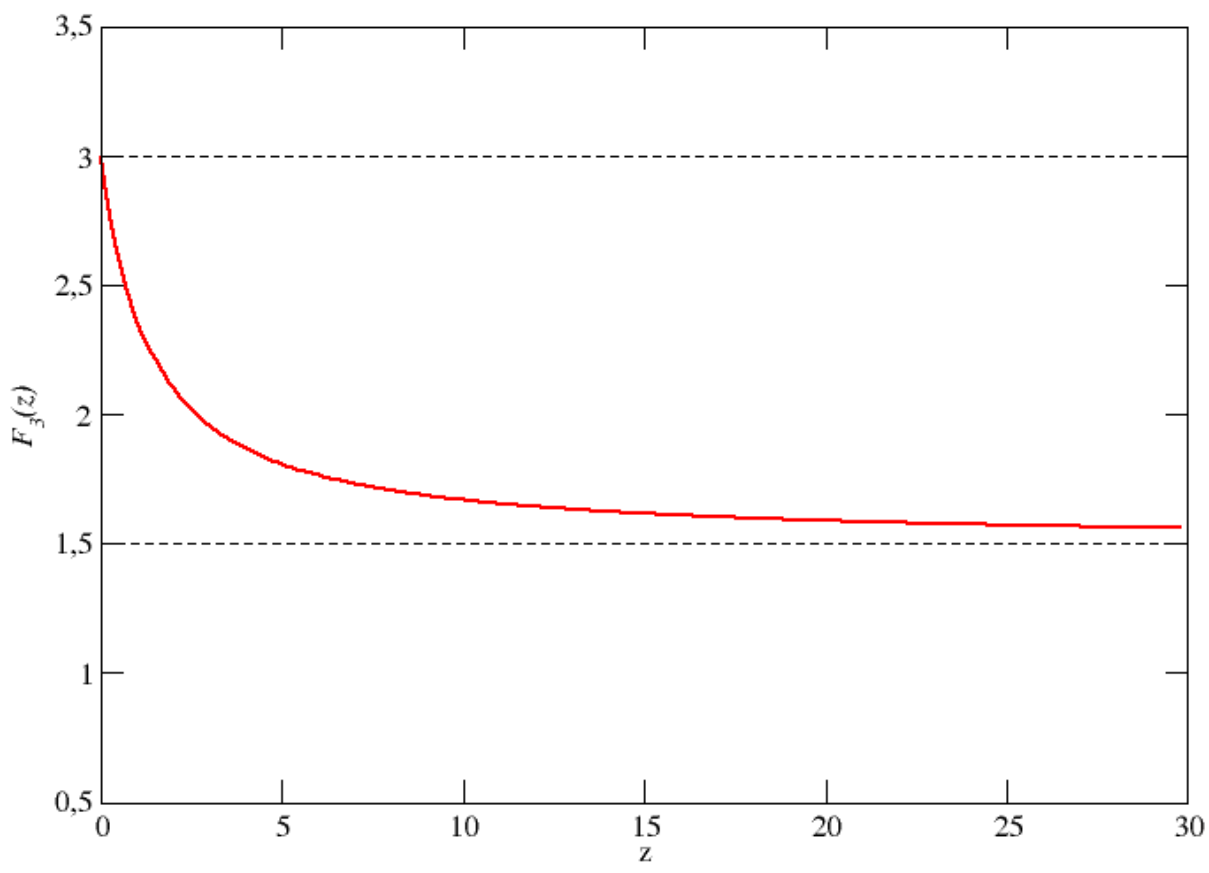

Figura 3.4: La gráfica muestra $\mathcal{F}_{d}(z)$ vs. $z=m c^{2} / k T$ para $d=3 . \mathcal{F}_{3}(z)$ corresponde al cociente entre el promedio de la energía cinética relativista por partícula y la temperatura comóvil. Vemos que en los casos $z \gg 1$ y $z \ll 1$ obtenemos los límites adecuados, mientras que para valores intermedios de $z$, el cociente es de una fracción de entre $3<\mathcal{F}_{3}(z)<3 / 2$.

De las expresiones (3.66)-(3.67), es claro que la temperatura y la energía no son simplemente proporcionales como en el caso no relativista, la energía relativista resulta ser una función complicada de $T$ y la temperatura no es una medida directa de la energía cinética del gas. Sin embargo, de (3.67), la temperatura es útil como un parámetro que determina en qué medida el sistema necesita ó no, una descripción relativista. Por otra parte a partir de (3.66) podemos reconocer $T$ como el promedio de una cantidad microscópica que es parte de la energía relativista como vemos en (3.67); de hecho, en el marco comóvil (3.66) conduce a

$$
k T=\frac{c}{d}\left\langle\left\langle\frac{|\mathbf{p}|^{2}}{p^{0}}\right\rangle\right\rangle
$$

la ecuación (3.69) es precisamente la expresión utilizada por Tolman [78] y Landsberg [70]. Esta expresión se ha malinterpretado queriendo identificar a $p^{0}$ como una masa en movimiento. En el límite no relativista, 
podemos aproximar $T$ como

$$
\frac{d}{2} k T=\langle\langle\mathcal{K}\rangle\rangle-\left\langle\left\langle\frac{\mathcal{K}^{2}}{m c^{2}}\right\rangle\right\rangle+\ldots,
$$

donde $\mathcal{K}$ es la energía cinética no relativista.

Notemos que, si bien versiones del teorema de equipartición relativista habían sido tratados con anterioridad por otros autores $[5,90]$ su enfoque no era manifiestamente covariante. Su trabajo y el nuestro coinciden en términos de la función $\mathcal{F}_{d}(3.67)$, en particular al evaluar los casos límite no-relativista y ultra-relativista. Cabe destacar que recientemente unos cálculos numéricos adoptando el método de Monte Carlo [22] confirman $\mathcal{F}_{d}$ como la energía cinética relativista en unidades de $k T$.

El teorema manifiestamente covariante (3.66)-(3.67) se obtuvo multiplicando el momento relativista $\mathcal{G}^{\mu}$ por $\Theta_{\mu}$, y restando la energía en reposo total $\mathcal{N} m c \Theta$ para obtener la energía cinética promedio de las partículas del gas. Es fácil ver que (3.66) puede reescribirse como

$$
\begin{aligned}
\Theta_{\mu} \mathcal{K}^{\mu} & =\mathcal{N} \mathcal{F}_{d}(m c \Theta), \\
\mathcal{K}^{\mu} & \equiv \mathcal{G}^{\mu}-\mathcal{N} m \mathcal{U}^{\mu},
\end{aligned}
$$

es claro que el momento relativista del gas tiene dos contribuciones. Podemos interpretar el término $\mathcal{K}^{\mu}$ como un 4-momento análogo a la contribución cinética de la energía no relativista, es decir, la parte cinética del momento total.

Si hubiéramos utilizado (3.44) como el impulso del gas, tendríamos que el producto $\Theta_{\mu} \mathcal{G}^{(\mu)}$ es

$$
\Theta_{\mu} \mathcal{G}^{(\mu)}=\mathcal{N F}_{d}(m c \Theta) \frac{\Theta^{0}}{\Theta}+\mathcal{N} m c \Theta^{0}
$$

que sólo coincide con (3.66)-(3.67) en el marco comóvil del gas donde $\Theta^{0}=\Theta$. Esto no es sorprendente ya que $\mathcal{G}^{(\mu)}$ es un vector aparente y no transforman correctamente bajo transformaciones de Lorentz. Podemos explorar el teorema de equipartición en el cono de luz pasado de un evento $\mathcal{E}$, como se propone en [23]

$$
\Theta_{\mu} \mathcal{G}_{L C}^{\mu}=\mathcal{N}\left(\mathcal{F}_{d}(m c \Theta)+m c \Theta\right)\left(\frac{\Theta^{0}}{\Theta}-\frac{\Theta_{i} \xi^{i}}{\Theta \xi}\right)
$$

Esta expresión depende del evento $\mathcal{E}$ elegido, lo cual es algo inusual. Si el evento está en el origen, entonces ((3.74) se reduce a (3.73), la ecuación (3.74) coincide con (3.66) sólo en el marco comóvil.

\subsection{Discusión}

El interés en combinar los principios de la relatividad en la teoría cinética va más allá de los fundamentos teóricos: las observaciones y experimentos recientes como por ejemplo en la física de altas energías [34], en astrofísica [24] y cosmología [67]. En todos estos casos se necesita una descripción de sistemas relativistas de muchas partículas en términos de, por ejemplo, la ecuación de Boltzmann y la correspondiente distribución 
del equilibrio [27, 25, 31]. Recientemente, Cubero et al. [19] (véase también las referencias allí citadas), llevaron a cabo simulaciones numéricas basadas en la dinámica molecular cuyos resultados apuntan a que la distribución de Jüttner es la distribución correcta en el equilibrio, para cualquier número de dimensiones espaciales, a diferencia de otras propuestas que hay en la literatura. Esto fue confirmado también para dos [20, 21] y tres dimensiones espaciales [23], [22], este último utilizando simulaciones de Monte Carlo. En el ámbito computacional, también hay intentos de extender al caso relativista el método conocido como Lattice Boltzmann, el cual considera aspectos generales de la ecuación de Boltzmann para la evolución de fluidos [91]. En el trabajo de Cubero et al. [19], el problema de la transformación relativista de la temperatura [69] se consideró en relación con una versión anterior del teorema de equipartición relativista propuesto por Landsberg [70]. Con base en este teorema Cubero et al. determinan que la temperatura debe poseer un carácter invariante. Dicho análisis no fue formulado en una forma manifiestamente covariante y, de hecho, era necesario algo más para interpretar ciertas contribuciones que aparecen en esa forma del teorema, así como el carácter de la temperatura ante cambio de marco de referencia.

En este trabajo hemos obtenido una forma manifiestamente covariante de la función de distribución relativista de Jüttner(3.41), a partir de una nueva deducción. En esta derivación utilizamos coordenadas cartesianas en un espacio de momentos de $(d+1)$ dimensiones ( $d$ dimensiones espaciales) en contraste con los resultados conocidos usando coordenadas esféricas. Esto fue posible gracias al uso del teorema de multiplicación de las funciones de Bessel (3.26) que simplificó el tratamiento de una serie de funciones de Bessel [82].

En este formalismo del carácter relativista de la temperatura no se establece desde un principio: Debido a la suposición de que el equilibrio en teoría cinética relativista debería estar de acuerdo con la termodinámica estándar para un observador comóvil con el sistema en reposo del gas, la pseudonorma invariante, $\Theta$, del 4-vector $\Theta^{\mu}(3.30)$, se convierte en $\Theta=c / k T$, (3.40), siendo $T$ la temperatura comóvil del gas. La temperatura es invariante de la misma forma que la masa en reposo de una partícula puntual. Una discusión similar a la que hay sobre el carácter de $T$ bajo transformaciones de Lorentz [69, 70, 19, 23], pero en el caso de la masa de una partícula puntual, plantea la cuestión de si tiene sentido distinguir entre masa en reposo y masa en movimiento o relativista [92]. La respuesta que da Okun en las referencias [92], es que sólo tiene sentido hablar de $m$ la masa de la partícula, siendo ésta una cantidad independiente del marco de referencia y asociada con la norma del 4-momento $p^{\mu} p_{\mu}=m^{2} c^{2}$. La confusión entre ambos términos aparece cuando se utilizan indistintamente la definición no relativista y relativista del momento. Los experimentos conocidos que dicen medir la variación de la masa relativista, en realidad describen un experimento para determinar las expresiones relativistas correctas para el momento espacial y la energía de partículas en movimiento [93]. Con la temperatura pasa algo similar.

El enfoque que aquí presentamos muestra explícitamente a la simetría de Lorentz como una simetría del sistema. Como sabemos las simetrías son importantes ya que están relacionadas con leyes de conservación (Teorema de Noether [36]). En mecánica estadística no relativista la convención estándar es considerar 
sistemas macroscópicamente estacionarios, es decir, en un marco de referencia donde tanto su momento espacial y angular son cero y no aparecen en ningún momento en el análisis, es siempre en el marco comóvil al sistema donde se trabaja y se tienen resultados. Según Callen [36] la forma más apropiada de leyes de la termodinámica es aquella en donde aparezcan manifiestamente todas las simetrías del sistema. En el caso relativista esto es claro y el formalismo aquí presentado exhibe la simetría manifiestamente. Sin embargo, hace falta un experimento para confirmar los resultados de nuestro enfoque y discriminar entre las demás posibilidades existentes en la literatura [69, 70, 19, 23]. Por ejemplo, de la misma forma que con la masa de una partícula, se requieren experimentos que determinen las expresiones para las componentes espaciales y temporal del vector térmico $\Theta^{\mu}$ para un gas en movimiento, además dichos experimentos deben dar un sentido más amplio al significado físico de las componentes de $\Theta^{\mu}$. Para enfrentar este problema hasta ahora sólo contamos son experimentos y simulaciones numéricas [19, 20, 22, 23, 91] cuya interpretación depende de la expresión del teorema de equipartición que se escoja.

En este capítulo también desarrollado un teorema de equipartición manifiestamente covariante dado por la ecuación (3.66), en la que el promedio de la energía-momento del gas determinado por el observador comóvil, está dado por la función $\mathcal{F}_{d}$ de la temperatura invariante, explícitamente (3.67). En efecto, esta expresión resulta análoga a la expresión para la energía en el caso de una partícula puntual, donde la energía se obtiene mediante la proyección del 4-momento a lo largo de la 4-velocidad del observador. En este caso tenemos el vector térmico $\Theta^{\mu}=\frac{c}{k T} \mathcal{U}^{\mu}$, con $\mathcal{U}^{\mu}$ la 4-velocidad del gas en su conjunto, que define un observador comóvil el cual mide la temperatura invariante $T$. Hay que hacer un comentario adicional sobre la diferencia entre nuestro enfoque y algunos anteriores. Mientras que las versiones anteriores del teorema de equipartición $[70,78]$ relacionan la temperatura con una combinación peculiar de cantidades relativistas (Véase, por ejemplo (3.63)), aquí esta combinación se interpreta como una parte de (3.66) que relaciona la temperatura invariante con el momento relativista promedio, $\mathcal{G}^{\mu}$. En resumen, la forma manifiestamente covariante de la distribución de Jüttner conduce naturalmente a considerar la temperatura comóvil invariante para caracterizar el régimen de equilibrio.

En este punto es importante recordar que la distribución relativista de equilibrio de Jüttner en la forma (3.1) o (3.41) no considera el carácter cuántico ni el grado de degeneración de las partículas que forman el gas. Estas características pueden tomarse en cuenta en una generalización a las distribuciones cuánticas para bosones y fermiones como discutiremos más adelante. Por esta razón el régimen de aplicación de (3.41) es limitado [97]. Por ejemplo, una enana blanca se modela como un gas de fermiones completamente degenerado, un resultado interesante de este modelo es que la masa de una enana blanca no puede exceder 1.4 veces la masa del Sol, a esto se conoce como el límite de Chandrasekhar [13, 24].

Sin embargo, la distribución de Jüttner es muy útil como la base del desarrollo de soluciones fuera de equilibrio, así como modelos que aproximan el término de colisión, como los que se mencionan en el siguiente capítulo. Además, ha servido para estudiar sistemas más complicados como mezclas de especies reactivas, ondas de choque y muy importante, el caso gravitacional [13]. Recientemente se ha generalizado 
la distribución de Jüttner para un gas en un marco de referencia acelerado [94] y en uno rotación [95]. Como un ejemplo de la manera de aplicar nuestros resultados en casos conocidos, consideremos la radiación de cuerpo negro. Recordemos el análisis estándar de este problema [27, 67, 75]. En primer lugar se considera el cociente entre la intensidad específica $I_{\nu}$ y el cubo de la frecuencia $\nu$ de los fotones. Dada la invariancia de Lorentz de esta cantidad $\frac{I_{\nu}}{\nu^{3}}$, [75], se sigue la invariancia de la distribución de Planck

$$
\frac{I_{\nu}}{2 h \nu^{3}}=\frac{1}{\mathrm{e}^{\frac{h \nu}{k T}}-1} .
$$

De hecho, para dos marcos en movimiento relativo, debemos tener

$$
\frac{h \nu}{k T}=\frac{h \nu^{\prime}}{k \mathcal{T}^{\prime}}
$$

donde las cantidades primadas se refieren a un observador que se mueve con respecto a la radiación, en particular, se sugiere que $\mathcal{T}^{\prime}$ es una temperatura no comóvil. Dado que los fotones sufren un desplazamiento Doppler se sigue que

$$
\frac{\nu}{\nu^{\prime}}=\gamma(1-\beta \cos \delta)
$$

donde $\delta$ es el ángulo entre el impulso del fotón y la velocidad del gas, mientras que $\beta$ es la relación entre la velocidad del gas y $c$. La cantidad $\mathcal{T}^{\prime}$ adopta una forma anisotrópica

$$
\mathcal{T}^{\prime}=\frac{T}{\gamma(1-\beta \cos \delta)}
$$

Podemos notar que, alternativamente, podríamos escribir una forma manifiestamente invariante para (3.75) como $\left(\mathrm{e}^{\Theta_{\mu} p^{\mu}}-1\right)^{-1}$. De esta manera podemos fijarnos en el producto invariante $\Theta_{\mu} p^{\mu}$. Mediante la evaluación en los dos sistemas de referencia mencionados anteriormente y utilizando que en el caso de fotones $p^{\prime 0}=\left|\mathbf{p}^{\prime}\right|$

$$
\frac{h \nu}{k T}=\Theta^{\prime 0} p^{\prime 0}(1-\beta \cos \delta) .
$$

Con la temperatura invariante comóvil $T$ los componentes de $\Theta^{\prime \mu}$ resultan ser $\frac{\gamma}{k T}(c, \mathcal{U})$ y por lo tanto (3.79) se reduce a (3.77). Esto demuestra la consistencia de adoptar la temperatura invariante comóvil, a través del vector $\Theta^{\mu}$ en la descripción de la radiación del cuerpo negro, sin recurrir a $\mathcal{T}^{\prime}$, ver ec. (3.78). Esta última expresión puede ser considerada sólo como una cantidad auxiliar por las siguientes razones: En primer lugar, las observaciones de la Radiación del Fondo Cósmico de Microondas (CMBR) [98] revelan que en realidad hay un marco de referencia en el que este presenta la estructura del cuerpo negro. Sin embargo, las mediciones involucran el brillo dependiendo de la frecuencia en lugar de una temperatura no comóvil (3.78). En segundo lugar, en el caso de las partículas masivas encontramos un obstáculo al intentar definir (3.78) (Véase, por ejemplo [99]). Para las partículas masivas tendríamos

$$
\begin{aligned}
\mathcal{T}^{\prime} & =\frac{T}{\gamma\left(1-\beta_{p} \beta \cos \delta\right)} \\
\beta_{p} & =\frac{|\mathbf{p}|}{p^{0}}
\end{aligned}
$$


que no tiene sentido físico debido a la dependencia en el momento de las partículas. Sin embargo la utilización de una temperatura comóvil invariante es viable por la misma razón que el caso anterior de los fotones funciona.

Nuestro trabajo se suma a las recientes afirmaciones elaboradas sobre la base de un detector de UnruhDeWitt [100], que apunta a la imposibilidad de tener una transformación relativista de la temperatura para la radiación [101]. Un análisis similar al presentado aquí se encuentra en un trabajo reciente de Nakamura [102]. Ahí se utiliza el vector térmico y se argumenta su utilidad en contraste con la temperatura direccional, como en (3.78). En cualquier caso, todos estos resultados refuerzan la idea de que la temperatura tiene sentido indiscutible en el marco comóvil.

Para estudiar de manera precisa la distribución de Planck, deberíamos considerar el límite ultra relativista de la distribución de equilibrio correspondiente a bosones, es decir debemos extender el estudio para que contenga las estadísticas cuánticas; como ya habíamos mencionado las aplicaciones más interesantes se encuentran en sistemas cuánticos degenerados $[12,13]$. Estas se toman en cuenta en la ecuación relativista de Uehling-Uhlenbeck [13, 96],

$$
\begin{aligned}
p^{\mu} \frac{\partial \bar{f}}{\partial x^{\mu}}+m \frac{\partial\left(\bar{f} \mathcal{K}^{\mu}\right)}{\partial p^{\mu}}= & \int\left[\bar{f} \bar{f}_{1}^{*}\left(1+\epsilon \frac{h^{3}}{g_{s}} \bar{f}\right)\left(1+\epsilon \frac{h^{3}}{g_{s}} \bar{f}_{1}\right)\right. \\
& \left.-\bar{f} \bar{f}_{1}\left(1+\epsilon \frac{h^{3}}{g_{s}} \bar{f}^{*}\right)\left(1+\epsilon \frac{h^{3}}{g_{s}} \bar{f}_{1}^{*}\right)\right] F \sigma_{Q} d \Omega \frac{d^{3} p_{1}}{p_{1}^{0}}
\end{aligned}
$$

donde $g_{s}$ es el factor de degeneración de espín y $\sigma_{Q}$ es la correspondiente sección transversal que se calcula a través de la teoría cuántica de dispersión. Las demás cantidades son análogas a aquellas definidas en la ecuación (3.2). El valor $\epsilon=-1$ corresponde al caso de la estadística de Fermi-Dirac, $\epsilon=+1$ a la de Bose-Einstein y $\epsilon=0$ a la estadística clásica. De la misma forma que en el caso clásico usual estudiado aquí podemos obtener la distribución del equilibrio, anulando el término de colisión del lado derecho de (3.81), lo que lleva a la siguiente condición

$$
\ln \frac{f}{1+\epsilon \frac{h^{3}}{g_{s}}}=-\left(\Lambda+\Theta_{\alpha} p^{\alpha}\right) \Leftrightarrow f=\frac{g_{s} / h^{3}}{\exp \left(\widetilde{\Lambda}+\Theta_{\alpha} p^{\alpha}\right)-\epsilon},
$$

donde $\widetilde{\Lambda}=\Lambda+\ln g_{s} / h^{3}$. De la misma forma en que identificamos la temperatura en el marco comóvil, se ha mostrado que en el marco comóvil el escalar anterior es $\widetilde{\Lambda}=\frac{\mu}{k T}$, donde $\mu$ es el potencial químico comóvil [13], de esta forma la distribución resultante es

$$
f=\frac{g_{s} / h^{3}}{e^{\Theta_{\alpha} p^{\alpha}-\frac{\mu}{k T}}-\epsilon} .
$$

Donde se ha identificado la norma de $\Theta^{\mu}$ con la temperatura invariante en un sistema comóvil con el gas. Ahora bien, como la temperatura está relacionada con la norma de un 4-vector podemos pensar que el vector térmico puede factorizarse como $\widetilde{\Lambda}=\Theta^{\alpha} \mathcal{M}_{\alpha}$, donde introducimos

$$
\mathcal{M}^{\alpha}=\frac{\mu}{c^{2}} \mathcal{U}^{\alpha}
$$


cuya norma está relacionada con el potencial químico en el marco comóvil $\mathcal{M}^{\alpha} \mathcal{M}_{\alpha}=\mu^{2} / c^{2}$. En particular para la estadística de Jüttner $\epsilon=0$ obtenemos

$$
\mathcal{M}^{\alpha}=\frac{k T}{c^{2}} \ln \left[\alpha h^{d} g_{s}^{-1}\right] \mathfrak{U}^{\alpha},
$$

donde $\alpha$ está definida por (3.29). La existencia de $\mathcal{M}^{\alpha}$ implica que para aumentar el número de partículas del sistema, es necesario considerar, además del potencial químico, el movimiento de este como un todo. Como para fotones $\mu_{\gamma}=0$, la discusión anterior sobre la distribución de Planck se mantiene.

La suposición de la existencia de $\mathcal{M}^{\alpha}$ debe estar de acuerdo con las posibles generalizaciones a las leyes de la termodinámica.

Esta versión manifiestamente covariante de la teoría cinética ofrece un formalismo que, además de provenir de la dinámica de los constituyentes microscópicos, parece no tener ambigüedades en la definición de cantidades macroscópicas, como en algunos trabajos sobre termodinámica relativista [72, 83, 71, 103]. De este modo podemos discutir la posibilidad de generalizar la forma de Gibbs de la primera ley de la termodinámica que en el caso no relativista está dada por (3.31). Es claro que la diferencial en el espacio termodinámico de la entropía (3.38), en un marco no comóvil nos dará

$$
\delta \mathcal{S}=k\left[\Theta_{\mu} \delta \mathcal{G}^{\mu}-\mathcal{N} \frac{N_{\mu} \delta N^{\mu}}{N_{\nu} N^{\nu}}\right]
$$

que se reduce a (3.31) en el límite no relativista en el marco comóvil utilizando la relación $\delta V=$ $-c\left(\mathcal{N} N^{\mu} \delta N_{\mu}\right) /\left(N^{\nu} N_{\nu}\right)^{3 / 2}$. Podemos considerar la ecuación (3.86) como una generalización manifiestamente covariante de la forma Gibbs de la segunda ley de la termodinámica obtenida a partir de la teoría cinética relativista.

Es interesante mencionar, entre las generalizaciones relativistas a la primera ley de la termodinámica, se encuentran algunos trabajos, [71], [87], [103], [104] donde se introduce un vector de volumen que apunta en la dirección de la velocidad del gas $V^{\mu} \equiv V \mathcal{U}^{\mu} / c$, tal que su norma es $V=\sqrt{V_{\mu} V^{\mu}}$. Además, se puede demostrar que $\delta V^{\mu}=-\left(c \mathcal{N} \delta N_{\mu}\right) / N^{\nu} N_{\nu}$, y junto con el hecho de que $\Theta^{\mu}$ apunta en la misma dirección que $N^{\mu}$, podemos factorizar el vector térmico en (3.86) de tal forma que obtenemos

$$
\delta \mathcal{S}=k \Theta_{\mu}\left[\delta \mathcal{G}^{\mu}+\frac{\mathcal{P}}{c} \delta V^{\mu}\right]
$$

que es una expresión utilizada por otros autores como una forma covariante de la expresión de Gibbs para la segunda ley de la termodinámica [103]. Otras versiones de las leyes de la termodinámica relativista se encuentran, por ejemplo en [72], [83], [71], [102]. Sin embargo, ambas expresiones (3.86) y (3.87), provienen de una teoría microscópica, y puede considerarse que tienen una base fuerte respecto a otras opciones que son una extrapolación de la fenomenología de la termodinámica comóvil. Por ejemplo, es fácil ver que la introducción de un potencial químico y su correspondiente verctor añadirían a (3.86)-(3.87) un término de la forma $\Theta^{\mu} \mathcal{M}_{\mu} \delta \mathcal{N}$. 
Como hemos visto en este capítulo, y como se afirma en [87], [23], es importante destacar que las propiedades de transformación de las variables termodinámicas dependen del carácter vectorial de $\mathcal{G}^{\mu}$. En ese sentido la versión manifiestamente covariante puede jugar un papel importante para hallar una generalización adecuada relativista de las leyes de la termodinámica. Sin embargo, lo que realmente dirá cuál es la generalización correcta es el experimento.

Hay varias posibles extensiones del presente trabajo que pueden ser de interés.

Una es ampliar el análisis del presente trabajo para el caso de la ecuación relativista de Fokker-Planck, por ejemplo en la misma línea de [105], este punto se discutirá en el siguiente capítulo.

Hemos visto que para resolver la ecuación de Boltzmann en el caso de la distribución de equilibrio, es necesario introducir el concepto de movimientos rígidos. Estos pueden relacionarse con las simetrías del espacio-tiempo de Minkowski que hemos considerado aquí y particularmente a la existencia de vectores de Killing. El caso donde se consideran además de traslaciones rotaciones también se ha estudiado [106], en ese caso el vector térmico tendrá contribuciones correspondientes al momento angular 4-dimensional.

En algunos trabajos se han estudiado espacios-tiempos curvos simétricos por ejemplo, en [10, 25], sin embargo, no parece haber ningún intento de incorporar el análogo del teorema de Noether en lo que respecta a la teoría cinética, que en particular si notamos que este último puede vincularse a la teoría cuántica de campo relativista $[12,77]$. Esto sería particularmente útil en el lenguaje manifiestamente covariante desarrollado aquí.

La teoría cinética relativista ha servido para estudiar sistemas fuera de equilibrio. En colisiones de núcleos pesados es posible obtener los coeficientes de transporte a partir de la ecuación de transporte (3.81) para las estadísticas cuánticas [107]. Los efectos de la viscosidad de bulto desempeñan un papel determinante en los procesos de producción de entropía en el universo temprano [25, 27, 28, 107, 108]. Asimismo, los términos de relajación en las leyes de transporte se han utilizado para interpretar las explosiones o implosiones rápidas en procesos astrofísicos como el colapso rápido anterior a la formación de estrellas de neutrones, algunos modelos de brotes de de rayos X, etc. [107]. Sería muy interesante aplicar el formalismo manifiestamente covariante a este tipo de sistemas.

En general, los enfoques covariantes a la mecánica estadística se han utilizado también para investigar los sistemas de muchas partículas en teorías relativistas generales [37]. Estudian sistemas con constricciones semejantes a las de la relatividad general, buscando posibles efectos estadísticos a partir de una dinámica microscópica cuántica. En este estudio aparece también un vector térmico asociado con el inverso de la temperatura cuando se elige una parametrización temporal particular. Sería muy interesante incorporar ideas similares a la teoría cinética, partiendo de cantidades invariantes ante reparametrizaciones temporales. Asimismo, la noción misma de espacio-tiempo se ha considerado en estos términos [42, 41, 109].

Por otra parte, existen teorías alternativas a la relatividad especial que se proponen para resolver ciertos problemas que tienen su origen en la gravitación cuántica. Una de estas teorías considera la variación 
de la velocidad de la luz (VSL) como una opción frente al modelo inflacionario de la cosmología. En ellas se ha estudiado cómo se modificaría la termodinámica relativista al tener una velocidad de la luz variable [110], resultando que el campo escalar asociado con la modificación de $c$ puede introducirse como una variable termodinámica en una generalización propuesta de la primera ley de la termodinámica. Otra modificación a la relatividad especial se logra, manteniendo $c$ constante, pero se introduciendo una escala de energía (típicamente la energía de Planck) que se considera invariante de Lorentz. A esta propuesta se le conoce como relatividad especial doble o deforme (DSR). Esto surge ya que en varias propuestas de gravedad cuántica esta escala juega un papel fundamental. Una consecuencia de esto es que el correspondiente espacio-tiempo es no conmutativo. En este escenario también se han explorado efectos en la termodinámica relativista donde la ecuación de estado del gas ideal se ve modificada por el parámetro no conmutativo de la DSR [111]. Resultaría interesante extender la teoría cinética relativista introduciendo las modificaciones que proponen estos modelos; esto proporcionaría un enfoque alternativo a la termodinámica de [110] y [111] introduciendo, además cantidades como el vector térmico que permiten una formulación manifiestamente covariante. 


\section{Ecuación de Fokker-Planck relativista: Mezclas binarias semirelativistas y gas simple}

En este capítulo se estudia la ecuación de Fokker-Planck relativista como aproximación del término de colisión de la ecuación de Boltzmann relativista para dos sistemas, el gas de Rayleigh o movimiento Browniano y el gas de Lorentz relativista. Ambos sistemas pueden considerarse complementarios. El gas de Lorentz consiste en una mezcla binaria compuesta de partículas ligeras relativistas que se difunden en un gas de fondo formado por partículas pesadas no relativistas, de modo que $m \ll m_{G}$ y $n \ll n_{G}$, con el sufijo $G$ indicando la componente abundante que forma el gas de fondo, $m$ y $n$ indican las masas y densidades de las partículas en reposo, respectivamente. El gas de Rayleigh es precisamente lo opuesto, una mezcla binaria donde la espacie abundante está formada por partículas ligeras relativistas, mientras que la especie que se difunde es escasa, y está formada por partículas pesadas no relativistas de modo que $m_{G} \ll m_{B}$ y $n_{B} \ll n_{G}$, donde el sufijo ${ }_{B}$ se refiere a las partículas Brownianas. Se considera la ecuación de Boltzmann relativista para la componente escasa, mientras que el gas de fondo $G$ se supone siempre en equilibrio.

La ecuación de Boltzmann se aproxima por un operador diferencial tipo Fokker-Planck, en donde pueden reconocerse los coeficientes de transporte. En el caso del gas de Lorentz relativista obtenemos un término adicional proporcional a la función de distribución además de la contribución habitual de Fokker-Planck. En ambos casos exploramos los casos límite el no relativista y el ultra relativista.

Además, se obtiene una ecuación de Fokker-Planck manifiestamente covariante para el gas de una componente, utilizando las aproximaciones de pequeña transferencia de momento y colisiones rasantes para desarrollar la integral de colisión. Se da también una expresión covariante para la relación fluctuacióndisipación entre los coeficientes de transporte. 


\subsection{Introducción}

Una manera de estudiar las ecuaciones cinéticas es a través de aproximaciones en el término de colisión. Este es el caso de los modelos de Marle [112], Anderson-Witting [113] que son las generalizaciones relativistas del modelo BGK (Bhatnagar, Gross y Krook) [114], donde la integral de colisión se sustituye por una frecuencia de colisión. Una aproximación alternativa para la integral de colisión consiste en utilizar un operador diferencial que modela un proceso de difusión. En el caso relativista la difusión se ha propuesto para estudiar la evolución de la materia oscura en el universo [115], la difusión en RHIC y LHC [116], así como correcciones relativistas al efecto Sunyaev-Zeldovich [32].

Hasta ahora ha habido varias propuestas distintas del movimiento browniano relativista [117], sin embargo, incluso la interpretación física de estos procesos es todavía confusa y aún no parece haber ningún acuerdo general [118].

Es interesante notar que el movimiento browniano relativista se ha utilizado en algunas propuestas de la gravedad cuántica, donde una escala de longitud mínima, posiblemente la longitud de Planck, juega un papel clave. Esto ha llevado a enfoques fenomenológicos que buscan revelar los efectos de tal longitud mínima, en ellos se sustituyen a las trayectorias de las partículas libres por procesos difusivos en el espaciotiempo [41]. En otras propuestas se tiene una ecuación estocástica utilizada para modelar los efectos de la micro-estructura del espacio-tiempo, asumiendo una métrica fluctuante [42].

Este capítulo está dedicado al estudio del proceso de difusión guiado por colisiones en lugar de por fuerzas estocásticas, es decir, a través de aproximar del término de colisión de la ecuación de Boltzmann relativista para dos mezclas binarias diferentes y complementarias. El gas de Rayleigh, que es un sistema con condiciones similares a las del movimiento Browniano, es decir, partículas pesadas no relativistas difundiéndose en un gas de partículas ligeras relativistas y el gas de Lorentz que consiste en partículas ligeras relativistas que se difunden en un gas de partículas pesadas no-relativistas. En ambos casos consideramos la evolución de la función de distribución. A través de algunas hipótesis simplificadoras el término de colisión de la ecuación de Boltzmann puede aproximarse por un operador diferencial tipo Fokker-Planck, en donde pueden reconocerse los coeficientes de transporte.

Se hace también un análisis del gas de una componente en la aproximación de pequeña transferencia de momento y colisiones rasantes, que también permite una descripción tipo Fokker-Planck, pero en este caso se mantiene la covarianza manifiesta, y se expresan así los correspondientes coeficientes de transporte.

\subsection{Ecuación de Boltzmann relativista para una mezcla binaria}

Para un gas simple compuesto por partículas de la misma especie que interactúan por pares a través de colisiones, es posible escribir la ecuación de Boltzmann relativista calculando la diferencia en el número de partículas que entran y salen de un elemento diferencial de volumen debido a las colisiones. Si partículas, 
con momento $p^{\mu}$ y $p_{*}^{\mu}$, respectivamente, sólo interactúan a través de colisiones elásticas, podemos utilizar la conservación del 4-momento para describir la colisión $p^{\mu}+p_{*}^{\mu}=p^{\prime \mu}+p_{*}^{\prime \mu}$, donde las cantidades primadas indican que se evalúan después de la colisión.

Una característica esencial en el caso relativista es la necesidad de considerar las propiedades de transformación correctas para las cantidades correspondientes ante transformaciones de Lorentz. Por ejemplo, la función de distribución relativista $f$ es un invariante de Lorentz [71, 74]. Esto puede verse porque el número de partículas en el elemento de volumen no cambiará ante un cambio de marco de referencia. También se puede demostrar [75], utilizando argumentos de simultaneidad y la restricción de la capa de masa $p^{\mu} p_{\mu}=m^{2} c^{2}=\left(p^{0}\right)^{2}-|\mathbf{p}|^{2}$, que el elemento de volumen en el espacio de fase es invariante $d^{3} x d^{3} p=d^{3} \tilde{x} d^{3} \tilde{p}$, donde la tilde se refiere a cantidades vistas en el marco de referencia en movimiento.

Para un gas con partículas de masa $m$, cuatro-momento $p^{\mu}=\left(p^{0}, \mathbf{p}\right)$, y coordenadas espacio-temporales $x^{\mu}=(c t, \mathbf{x})$, la función de distribución de una partícula $f\left(x^{\mu}, p^{\mu}\right)=f(\mathbf{x}, \mathbf{p}, t)$ caracteriza el estado del sistema, da el número de partículas $d \mathcal{N}=f(\mathbf{x}, \mathbf{p}, t) d^{3} x d^{3} p$ al tiempo $t$ dentro del elemento de volumen $d^{3} x$ ubicado en $\mathbf{x}$ con momento $\mathbf{p}$ en el rango $d^{3} p$. La evolución de la función de distribución de una partícula $f$ está dictada por la ecuación de Boltzmann relativista $[12,13]$

$$
p^{\mu} \frac{\partial f}{\partial x^{\mu}}+m \frac{\partial f \mathcal{K}^{\mu}}{\partial p^{\mu}}=\int\left(f^{\prime} f_{*}^{\prime}-f f_{*}\right) F \sigma d \Omega \frac{d^{3} p_{*}}{p_{*}^{0}}
$$

donde $f_{*}^{\prime}=f\left(\mathbf{x}, \mathbf{p}_{*}^{\prime}, t\right) . \sigma$ es la sección eficaz diferencial del proceso de dispersión, mientras que $\Omega$ es el ángulo sólido. $\mathcal{K}^{\mu}$ denota una 4-fuerza externa mientras que $F$ es el llamado flujo invariante, $F=$ $\sqrt{\left(p_{1}^{\mu} p_{\mu}\right)^{2}-m^{4} c^{4}}$, y $\frac{d^{3} p_{1}}{p_{1}^{0}}$ es la medida invariante (en la capa de la masa). Estaremos interesados en el caso $\mathcal{K}^{\mu}=0$ correspondiente a no tener fuerzas externas. Al lado derecho de (4.1) se le conoce como integral de colisión. Escrita de esta manera, la ecuación de Boltzmann relativista es manifiestamente covariante. Hay otra forma equivalente que no es manifiestamente covariante y que utilizaremos a lo largo de este capítulo

$$
\frac{\partial f}{\partial t}+v^{i} \frac{\partial f}{\partial x^{i}}+\frac{\partial\left(f \mathrm{~F}^{i}\right)}{\partial p^{i}}=\int\left(f^{\prime} f_{*}^{\prime}-f f_{*}\right) g_{\varnothing} \sigma d \Omega d^{3} p_{*}=\mathcal{J}(f, f)
$$

donde $\mathrm{F}^{i}$ son las componentes espaciales de la fuerza externa que en particular serán cero $\mathrm{F}^{i}=0$, y $g_{\varnothing}$ es la velocidad de Møller [12, 13], que se relaciona con el flujo invariante a través de $g_{\varnothing}=\frac{c F}{p^{0} p_{*}^{0}}$ y se reduce a la velocidad relativa en el caso no relativista.

La evolución de una mezcla binaria está dictada por un sistema de ecuaciones de Boltzmann acopladas, una por cada componente, teniendo del lado derecho dos integrales de colisión, una por la interacción con partículas de la misma especie y otra que considera colisiones entre distintas especies

$$
\begin{aligned}
\frac{\partial f}{\partial t}+v^{i} \frac{\partial f}{\partial x^{i}} & =\mathcal{J}(f, f)+\mathcal{J}\left(f_{G}, f\right), \\
\frac{\partial f_{G}}{\partial t}+v_{G}^{i} \frac{\partial f_{G}}{\partial x^{i}} & =\mathcal{J}\left(f_{G}, f_{G}\right)+\mathcal{J}\left(f, f_{G}\right),
\end{aligned}
$$


Sin embargo, dadas las características de las mezclas que estudiamos aquí podemos hacer una suposición conocida del caso no relativista $[119,120]$ y que podemos extender al caso relativista. La ecuación de Boltzmann para la función de distribución de las partículas del gas no se ve afectada por la componente que se difunde debido a que siempre se considera muy escasa en comparación con la especie que constituye el gas de fondo, esta condición se expresa como $n \ll n_{G}$, donde ${ }_{G}$ indica el gas de fondo. Por tanto, suponemos que el gas se mantiene en equilibrio y el lado derecho de la ecuación (4.4) se anula. La ecuación para las partículas que se difunden contendrá únicamente el término correspondiente a interacciones entre partículas de distintas especies, ya que el término de interacción entre las mismas partículas se desprecia en (4.3) debido a que al ser escasas estas casi no interactúan. La ecuación de Boltzmann restante puede escribirse como

$$
\frac{\partial f}{\partial t}+v^{i} \frac{\partial f}{\partial x^{i}}+\frac{\partial\left(f \mathrm{~F}^{i}\right)}{\partial p^{i}}=f^{(0)} \int\left(h^{\prime}-h\right) f_{G}^{(0)} g_{\varnothing} \sigma d \Omega d^{3} p_{G}=f^{(0)} \mathcal{I},
$$

donde se supuso que la distribución de las partículas que se difunden se puede expresar como una desviación del equilibrio $f=f^{(0)} h(p)$. De esta forma se puede hacer un desarrollo de Taylor de la desviación $h^{\prime}=$ $h\left(p^{i}+\Delta p^{i}\right)$ evaluada después de la colisión, alrededor del momento antes de la colisión $p^{i}$, considerando términos hasta de segundo orden. De este modo la diferencia $h^{\prime}-h$ se escribe como:

$$
h^{\prime}-h \approx \Delta p^{i} \frac{\partial h}{\partial p^{i}}+\frac{1}{2} \Delta p^{i} \Delta p^{j} \frac{\partial^{2} h}{\partial p^{i} \partial p^{j}} .
$$

Para continuar es necesario introducir la velocidad relativa definida de la siguiente forma

$$
\mathrm{g}^{i} \equiv c \frac{p_{G}^{i}}{p_{G}^{0}}-c \frac{p^{i}}{p^{0}},
$$

con esta velocidad se calcula la diferencia antes y después de la colisión

$$
\mathrm{g}^{i^{\prime}}-\mathrm{g}^{i}=c\left(\frac{p_{G}^{i^{\prime}}}{p_{G}^{0}}-\frac{p^{i^{\prime}}}{p^{0}}\right)-c\left(\frac{p_{G}^{i}}{p_{G}^{0}}-\frac{p^{i}}{p^{0}}\right),
$$

de esta forma se aproximarán las diferencias en el momento de las partículas que se difunden en términos de diferencias en la velocidad relativa. Sin embargo, en cada caso la aproximación será diferente. Esto se debe en esencia a que el cociente entre las energías cambia en cada caso. Cuando una componente es relativista y la otra no, el cociente entre las energías es $p_{\text {rel }}^{0} / p_{\text {norel }}^{0} \ll 1$.

El paso final es identificar los coeficientes de transporte como las integrales de ciertas cantidades que, de nuevo, en cada caso serán distintas. Estos serán simplemente los coeficientes del operador diferencial tipo Fokker-Planck que reemplazara la integral de colisión.

\subsection{Gas de Rayleigh - Movimiento Browniano relativista}

El término movimiento Browniano usualmente se refiere al movimiento irregular que una partícula de polen describe en un líquido, el cual fue observado por primera vez por el botánico escocés Robert Brown 
en 1827 [121]. Fue hasta 1905 que Einstein [122] y Smoluchowski [123] dieron bases teóricas a dichas observaciones. Esta teoría fue confirmada experimentalmente en 1909 por J. Perrín [124] dando además evidencia de la veracidad de la teoría atómica de la materia. El problema del movimiento Browniano ha sido fundamental desde entonces hasta aplicaciones actuales en química, biología y ciencias sociales [125]. Particularmente el movimiento Browniano relativista puede aplicarse en física de plasmas y astrofísica, por ejemplo en el análisis de los chorros relativistas, en correcciones al efecto Sunyaev-Zeldovich [32], en la descripción de materia oscura [115] o en difusión en el plasma de quarks y gluones [34, 35].

En el caso no relativista el movimiento Browniano se estudia utilizando ecuaciones estocásticas en particular la ecuación de Langevin [126]. En este capítulo consideraremos el movimiento Browniano relativista desde el punto de vista de la teoría cinética, es decir, veremos que una aproximación adecuada de la ecuación de Boltzmann, utilizando las características del sistema, da lugar a un esquema equivalente al movimiento Browniano conocido como el gas de Rayleigh en el caso no relativista. Este enfoque nos da una alternativa a las generalizaciones relativistas de los procesos estocásticos como se hace en [117, 118], de tal forma que la evolución al equilibrio del sistema estará dirigida por colisiones en lugar que por términos estocásticos.

Para el caso del movimiento Browniano relativista [105] consideramos una mezcla binaria, donde uno de sus constituyentes se toma como un gas de fondo formado por partículas ligeras relativistas, mientras que la especie que se difunde se considera formada por partículas pesadas no relativistas, de tal forma que la relación entre sus masas satisface $m_{G} \ll m$ y sus densidades $n \ll n_{G}$. En la referencia [105] se encontró que la difusión de las partículas Brownianas pesadas no relativistas, en un gas de partículas ligeras relativistas, puede describirse con una generalización relativista de la ecuación de Fokker-Planck a partir de la ecuación de Boltzmann relativista (4.2). En este caso la relación entre las energías resulta ser $p_{G}^{0} / p_{B}^{0} \ll 1$, esto puede verificarse de la siguiente manera:

Primero se considera que al orden dominante la componente cero del momento de las partículas Brownianas es el mismo antes y después de la colisión $p_{B}^{0 \prime} \approx p_{B}^{0}$ y por la conservación de la energía se tiene que $p_{G}^{0 \prime} \approx p_{G}^{0}$. De esta manera la ecuación (4.8) se escribe como

$$
\mathrm{g}^{i^{\prime}}-\mathrm{g}^{i}=\frac{c}{p_{G}^{0}}\left(p_{G}^{i^{\prime}}-p_{G}^{i}\right)-\frac{c}{p_{B}^{0}}\left(p_{B}^{i^{\prime}}-p_{B}^{i}\right) .
$$

Por otro lado se tiene la conservación de las componentes espaciales del momento

$$
p_{G}^{i^{\prime}}-p_{G}^{i}=-\left(p_{B}^{i^{\prime}}-p_{B}^{i}\right) \text {. }
$$

Al sustituir (4.10) en (4.9) se llega a

$$
\mathrm{g}^{i^{\prime}}-\mathrm{g}^{i}=-c\left(\frac{1}{p_{G}^{0}}+\frac{1}{p_{B}^{0}}\right)\left(p_{B}^{i^{\prime}}-p_{B}^{i}\right) .
$$

Esta ecuación es la más general para la diferencia entre las velocidades relativas en el caso estudiado. Para continuar es necesario introducir algunas aproximaciones en el término que involucra a las componentes temporales en la última expresión (4.11). 
Para aproximar es necesario recordar que la energía relativista de una partícula, es decir, la componente temporal $p_{B}^{0}$, puede escribirse en términos de su energía en reposo $m_{B} c^{2}$ y su energía cinética relativista $\mathcal{K}$ en la siguiente forma

$$
c p_{B}^{0}=m_{B} c^{2}+\mathcal{K}
$$

Por otra parte la energía relativista promedio de una partícula se puede obtener a partir del teorema de equipartición que estudiamos en el capítulo 3 , y está dado por la ecuación (3.67), que en $d=3$ dimensiones espaciales puede escribirse como

$$
\mathcal{K}=\mathcal{F}_{3}(z) k T
$$

donde

$$
\mathcal{F}_{3}(z)=z_{B} \frac{K_{3}\left(z_{B}\right)}{K_{2}\left(z_{B}\right)}-z_{B}-1
$$

donde como sabemos $z_{B}=m_{B} c^{2} / k T . \mathcal{F}_{3}(z)$ es función de la temperatura acotada por $\frac{3}{2} \leq \mathcal{F}_{3} \leq 3$. Las cotas corresponden a los casos límite. El caso no relativista se obtiene al suponer bajas temperaturas $z_{B} \gg 1$, que corresponde a $\mathcal{K}_{n o \text { rel }}=\frac{3}{2} k T$, mientras que el límite ultra relativista correspondiente a masas pequeñas (o nulas) o temperaturas altas $z_{B} \ll 1$ cuya energía es $\mathcal{K}_{\text {ultra }}=3 k T$. Con estos resultados podemos hacer algunas observaciones. En una mezcla donde una de las componentes es no relativista y la otra es un gas ultra relativista (semejante al caso estudiado en esta sección), se puede apreciar que, para tener la misma temperatura uno de los gases cede energía: $\mathcal{K}_{\text {ultra }}=2 \mathcal{K}_{n o \text { rel }}$, [5].

Ahora bien, en el caso relativista genérico podemos estimar el orden de magnitud de la energía cinética en relación con la temperatura usando los casos límite de $\mathcal{F}_{3}$. De esta manera se considerará la siguiente aproximación para la energía cinética relativista

$$
\mathcal{K} \approx k T,
$$

con esto la energía relativista (4.12) se escribe como

$$
c p_{B}^{0} \cong m_{B} c^{2}+k T \text {. }
$$

Es preciso recordar que en el caso de estudio ambas especies están a la misma temperatura T. Como las partículas pesadas que se difunden son no relativistas se cumple que

$$
k T \ll m_{B} c^{2} .
$$

Como están a la misma temperatura se puede sustituir la ecuación (4.16) para la energía de las partículas del gas en (4.17) llevando a

$$
c p_{G}^{0}-m_{G} c^{2} \ll m_{B} c^{2} \Rightarrow \frac{p_{G}^{0}}{m_{B} c} \ll 1+\frac{m_{G}}{m_{B}} .
$$


Dada la relación entre las masas $m \gg m_{G}$, el último término de (4.18) se desprecia y resulta la relación buscada $p_{G}^{0} / p_{B}^{0} \ll 1$. Se tiene entonces una relación entre componentes temporales del 4-momento. Ahora podemos aproximar (4.11) utilizando esta aproximación, con lo que obtenemos

$$
\Delta \mathrm{g}^{i}=-\frac{c}{p_{G}^{0}} \Delta p_{B}^{i} \quad \Rightarrow \quad \Delta p_{B}^{i}=-\frac{p_{G}^{0}}{c} \Delta \mathrm{g}^{i} .
$$

De esta forma el término integral (4.5) puede reemplazarse por un operador diferencial tipo-Fokker-Planck como una aproximación a la integral de colisión ${ }^{1}$ :

$$
\frac{\partial f_{B}}{\partial t}+v^{i} \frac{\partial f_{B}}{\partial x^{i}}+F^{i} \frac{\partial f_{B}}{\partial p^{i}}=\eta\left(m_{B} k T \frac{\partial^{2} f_{B}}{\partial p^{i} \partial p_{i}}+\frac{\partial\left(f_{B} p^{i}\right)}{\partial p^{i}}\right)
$$

donde $f_{B}$ es la función de distribución para las partículas Brownianas mientras que $\eta$ es el coeficiente de fricción-viscosidad que fue encontrado en términos de integrales de colisión de cantidades microscópicas propias del gas de fondo [105]

$$
\eta=\frac{2}{3} \frac{n_{G} \pi}{m_{B}\left(m_{G} k T\right)^{2} K_{2}\left(z_{G}\right)} \int \sigma\left(\left|\mathbf{p}_{G}\right|, \chi\right)(1-\cos \chi) \operatorname{sen} \chi d \chi e^{-\frac{u_{\alpha} p_{G}^{\alpha}}{k T}}\left|\mathbf{p}_{G}\right|^{5} \frac{d\left|\mathbf{p}_{G}\right|}{p_{G}^{0}} .
$$

La expresión (4.20) es idéntica a la ecuación de Fokker-Planck en el caso no relativista [119], [120], con la diferencia únicamente en la definición del coeficiente de fricción-viscosidad y el momento relativista. Además, esta ecuación es similar a aquella encontrada a partir de la generalización relativista de los procesos estocásticos [117], [118], por ello que llamamos al sistema movimiento Browniano relativista. Lo que expresa la expresión (4.21) es que el sistema formado por partículas relativistas y no relativistas, evoluciona por colisiones del mismo modo que lo haría un sistema donde las partículas se difunden.

Para el caso particular de una sección transversal $\sigma$ constante, el coeficiente de fricción-viscosidad $\eta$ está dado por [105]

$$
\eta=\frac{32 \pi n_{G} \sigma k T}{3 m_{B} c K_{2}\left(z_{G}\right)} \frac{e^{-z_{G}}}{z_{G}^{2}}\left(3+3 z_{G}+z_{G}^{2}\right)
$$

donde $K_{2}$ son las funciones de Bessel modificadas de segunda clase, y $z_{G}=m_{G} c^{2} / k T$ es el cociente entre la energía en reposo y la energía térmica $k T$.

En el caso no relativista de bajas temperaturas $z_{G} \gg 1$, se puede usar una expresión para la función de Bessel de segunda clase como una serie $^{2}$, así se obtiene la siguiente expresión para el coeficiente de fricción-viscosidad

$$
\eta_{N R} \cong \frac{32 n_{G} \sigma}{3 m_{B}} \sqrt{2 m_{G} \pi k T}\left(1+\frac{9}{8 z_{G}}+\frac{9}{128 z_{G}^{2}}-\frac{765}{1024 z_{G}^{3}}+\ldots\right) .
$$

El término dominante de la serie en (4.23) corresponde a la forma conocida para el movimiento browniano o gas de Rayleigh no relativista calculada previamente $[119,120]$ cuando $\sigma=d^{2} / 4$, con $d$ el diámetro de la esfera dura. Cabe mencionar que el inverso de $\eta$ se le llama también la movilidad, [126].

\footnotetext{
${ }^{1}$ Los detalles pueden consultarse en el apéndice D.1

${ }^{2}$ Ver ecuación (C.21) del apéndice C.
} 
En el límite ultra relativista el parámetro $z_{G} \ll 1$, condición satisfecha cuando la temperatura es muy alta, la función de Bessel puede aproximarse ${ }^{3}$ de la siguiente manera

$$
\eta_{U R} \cong \frac{16 \pi n_{G} \sigma k T}{m_{B} c}\left(1+\frac{z_{G}^{2}}{12}+\frac{z_{G}^{4}}{64}\left(1+4 \ln \left(\frac{z_{G}}{2}\right)+4 \gamma_{e}\right)-\frac{z_{G}^{5}}{45}+\ldots\right),
$$

donde $\gamma_{e}=0,577215664 \ldots$ es la constante de Euler. Podemos notar que la dependencia en la temperatura es distinta en cada caso (4.23) y (4.24).

Siguiendo el procedimiento que se describe en [127], obtenemos la solución a la ecuación de Fokker-Planck:

$$
f_{B}\left(p_{B}, t\right)=\frac{n_{B}}{\left[2 m_{B} \pi k T\left(1-e^{-2 \eta t}\right)\right]^{\frac{3}{2}}} \exp \left[-\frac{\left(p_{B}^{i}-p_{0_{B}}^{i} e^{-\eta t}\right)\left(p_{i_{B}}-p_{i_{0_{B}}} e^{-\eta t}\right)}{2 m_{B} k T\left(1-e^{-2 \eta t}\right)}\right],
$$

donde $p_{0_{B}}^{i}$ es el momento inicial. En el límite cuando $t \gg \eta^{-1}$ la ecuación (4.25) se reduce a la distribución de Maxwell-Boltzmann, es decir, $\eta^{-1}$ es el tiempo en que el sistema relaja al equilibrio. Notemos que las correcciones relativistas a la función de distribución de las partículas Brownianas (4.25) se encuentran en la correspondiente expresión para $\eta$. Si tomamos como ejemplo el caso no relativista (4.23) a primer orden, tendríamos que el tiempo de relajación se modifica

$$
\eta^{-1} \cong \eta_{0}^{-1}\left(1-\frac{9}{8} z_{G}^{-1}+\ldots\right)
$$

donde $\eta_{0}=\frac{32 n_{G} \sigma}{3 m_{B}} \sqrt{2 m_{G} \pi k T}$, es el término dominante del coeficiente de fricción-viscosidad no relativista. Podemos apreciar que la modificación relativista disminuye la magnitud del tiempo de relajación por un término de orden $z_{G}^{-1}$, dicho en otras palabras los efectos relativistas desaparecen pasado un tiempo de orden $z_{G}^{-1}$ que en ese límite es muy pequeño.

\subsection{Ecuación de Fokker-Planck para el gas de Lorentz relativista}

Consideremos ahora el gas de Lorentz [128, 129], es decir, una mezcla binaria donde uno de los constituyentes se considera como gas de fondo y está conformada por partículas pesadas no relativistas con masa en reposo $m_{G}$, y la velocidad de cada partícula $v_{G} \ll c$. La otra especie se considera relativista y ligera, es decir con masa pequeña en comparación con la masa del gas de fondo $m \ll m_{G}$, además la densidad es mucho menor que la del gas de fondo $n \ll n_{G}$. Claramente este sistema puede considerarse como el caso complementario al estudiado en la sección anterior donde partículas pesadas no relativistas se difunden en un gas de partículas ligeras relativistas.

Este sistema se ha utilizado para modelar un plasma débilmente ionizado o la termalización de partículas en un baño térmico [130, 131, 132]. Algunas versiones relativistas se han estudiado en [12, 133, 134] y [135], por ejemplo, para el caso de un haz de electrones en un plasma relativista. En [133] se hace un

\footnotetext{
${ }^{3}$ Ver ecuación (C.24) del apéndice C.
} 
análisis para identificar a una ecuación de Fokker-Planck para el gas de Lorentz relativista en el que la distribución es una función del 4-momento. Esto permite dividir de forma aditiva la dependencia de la energía y el impulso espacial; a continuación, cada parte obedece a una ecuación correspondiente FokkerPlanck. Los términos de la parte de la energía pueden ser identificados en el límite no relativista con el obtenido por Chapmann y Cowling [129].

En esta sección estudiaremos el sistema de manera análoga al sistema de la sección anterior. En [47], se encontró que la difusión de las partículas ligeras relativistas, en un gas de partículas pesadas no relativistas, puede describirse con una generalización relativista de la ecuación de Fokker-Planck a partir de la ecuación de Boltzmann relativista (4.2), siguiendo el mismo procedimiento que se utilizó al estudiar el sistema de la sección anterior. En este caso la relación entre las energías resulta ser $p^{0} / p_{G}^{0} \ll 1$, que es el inverso del caso de partículas Brownianas. Esto significa que la diferencia en el momento de las partículas que se difunden puede aproximarse de la siguiente forma

$$
\Delta \mathrm{g}^{i} \cong-c \frac{\Delta p^{i}}{p^{0}}
$$

Esta relación involucra sólo la componente cero del 4-momento de las partículas que se difunden en comparación con el movimiento browniano relativista discutido en la sección anterior para que en lugar de $p^{0}$ se tiene $p_{G}^{0}$. Ahora es necesario incorporar esta aproximación en (4.8) para determinar la integral $\mathcal{I}$. Esto se hace en detalle en el apéndice D.2. De los resultados del apéndice $\mathcal{I}$ se puede escribir como un operador diferencial:

$$
\mathcal{I}=-C^{i} \frac{\partial h}{\partial p^{i}}+D^{i j} \frac{\partial^{2} h}{\partial p^{i} \partial p^{j}}
$$

donde

$$
\begin{gathered}
C^{i} \equiv 2 \pi c \frac{|\mathbf{p}|}{p^{0}} n_{G} a_{1}\left[1+2\left(\frac{p^{0}}{|\mathbf{p}|}\right)^{2} \frac{k T}{m_{G} c^{2}}\right] p^{i}, \\
D^{i j} \equiv \frac{\pi n_{G}}{2} c \frac{|\mathbf{p}|}{p^{0}}\left\{\left[p^{i} p^{j}\left(4 a_{1}-3 a_{2}\right)-a_{2} \eta^{i j}|\mathbf{p}|^{2}\right]+\right. \\
\left.\frac{1}{2}\left(\frac{p^{0}}{|\mathbf{p}|}\right)^{2} \frac{k T}{m_{G} c^{2}}\left[5 p^{i} p^{j}\left(4 a_{1}-3 a_{2}\right)-\eta^{i j}|\mathbf{p}|^{2}\left(8 a_{1}+5 a_{2}\right)\right]\right\} .
\end{gathered}
$$

En la ecuación (4.29) y (4.30) se introdujeron las siguientes integrales

$$
\begin{gathered}
a_{1}(|\mathbf{p}|)=\int \sigma(|\mathbf{p}|, \chi)(1-\cos \chi) \sin \chi d \chi \\
a_{2}(|\mathbf{p}|)=\int \sigma(|\mathbf{p}|, \chi) \sin ^{3} \chi d \chi
\end{gathered}
$$

ambas contienen a la sección eficaz $\sigma(|\mathbf{p}|, \chi)$ y será necesario especificarla para obtener su valor explícito. Nuestro objetivo será aproximar la integral de colisión de la ecuación de Boltzmann (4.5) por un operador diferencial. Después de un cálculo largo pero elemental que consiste de reorganizar de las funciones $h$ y 
$f^{(0)}$ para expresar el producto $f^{(0)} \mathcal{I}$ en términos de $f$ y sus derivadas, se obtiene que el lado derecho de la ecuación de Boltzmann (4.5) se aproxima por la siguiente expresión:

$$
\frac{\partial f}{\partial t}+\frac{\partial}{\partial p^{i}}\left(F^{i} f\right)=D^{i j} \frac{\partial^{2} f}{\partial p^{i} \partial p^{j}}-A^{i} \frac{\partial f}{\partial p^{i}}-\nu f
$$

donde se definen

$$
\begin{gathered}
A^{i} \equiv C^{i}+\frac{2 c}{k T p^{0}} D^{i j} p_{j} \\
\nu \equiv \frac{c}{k T p^{0}}\left\{D^{i j}\left[\eta_{i j}+\frac{p_{i} p_{j}}{\left(p^{0}\right)^{2}}\left(1-\frac{c p^{0}}{k T}\right)\right]-C^{i} p_{i}\right\} .
\end{gathered}
$$

Haciendo uso de (4.29) y (4.30) tenemos que $A^{i}$ y $\nu$ pueden escribirse como:

$$
\begin{aligned}
& A^{i}=\tilde{\eta} p^{i} {\left[1-2\left(1-\frac{a_{2}}{a_{1}}\right) \frac{c p^{0}}{k T}\left(\frac{|\mathbf{p}|}{p^{0}}\right)^{2}-2\left(\frac{p^{0}}{|\mathbf{p}|}\right)^{2} \frac{k T}{m_{G} c^{2}}\right.} \\
&\left.-7\left(\frac{p^{0}}{p_{G}^{0}}\right)\left(1-\frac{5}{14} \frac{a_{2}}{a_{1}}\right)\right], \\
& \nu=\tilde{\eta} \frac{c p^{0}}{k T}\left(\frac{|\mathbf{p}|}{p^{0}}\right)^{2}\left\{\left(1-\frac{a_{2}}{2 a_{1}}\right)\left(1-\frac{c p^{0}}{k T}\right)\left(\frac{|\mathbf{p}|}{p^{0}}\right)^{2}\right. \\
&\left.+\frac{7}{2} \frac{k T}{m_{G} c^{2}}\left(1-\frac{5}{14} \frac{a_{1}}{a_{2}}-\left(\frac{p^{0}}{|\mathbf{p}|}\right)^{2}\right)-\frac{7}{2} \frac{p^{0}}{p_{G}^{0}}\right\},
\end{aligned}
$$

donde podemos factorizar el coeficiente de fricción-viscosidad $\widetilde{\eta}$ en función del momento de la difusión de partículas:

$$
\widetilde{\eta}(|\mathbf{p}|) \equiv 2 \pi n_{G} c \frac{|\mathbf{p}|}{p^{0}} a_{1}(|\mathbf{p}|)
$$

La ecuación (4.33) es una ecuación generalizada tipo Fokker-Planck, donde $D^{i j}$ es el tensor de difusión, $A^{i}$ es el vector de fricción dinámica, además de un término adicional proporcional a $f$ cuyo coeficiente $\nu$ puede asociarse a un frecuencia característica o un tiempo de relajación de manera similar que en el modelo BGK [114].

Todos los coeficientes (4.30), (4.36) y (4.37) son funciones de $|\mathbf{p}|$ y para determinarlos expresamente necesitamos especificar la sección eficaz diferencial. Vamos a utilizar el modelo de esferas duras donde $\sigma$ es constante ${ }^{4}$. Con esta aproximación a la integración en el ángulo $\chi$, las integrales en (4.31)-(4.32) pueden efectuarse dando como resultado

$$
a_{1}=2 \sigma \quad \mathrm{y} \quad a_{2}=\frac{4}{3} \sigma
$$

\footnotetext{
${ }^{4}$ Para una justificación física de esta aproximación véase, por ejemplo la elección de la sección transversal de la dispersión de fotones en un gas de electrones no relativistas en [12].
} 
Por lo tanto los coeficientes correspondientes para este caso son

$$
\begin{aligned}
D^{i j}=\widetilde{\eta}_{H S} & {\left[\left(p^{i} p^{j}-\frac{|\mathbf{p}|^{2}}{3} \eta^{i j}\right)+\frac{5}{4}\left(\frac{p^{0}}{|\mathbf{p}|}\right)^{2} \frac{k T}{m_{G} c^{2}} \times\right.} \\
& \left.\left(p^{i} p^{j}-\frac{58}{15}|\mathbf{p}|^{2} \eta^{i j}\right)\right], \\
A^{i}= & \widetilde{\eta}_{H S} p^{i}\left[1-\frac{2}{3} \frac{c p^{0}}{k T}\left(\frac{|\mathbf{p}|}{p^{0}}\right)^{2}-\left(\frac{p^{0}}{|\mathbf{p}|}\right)^{2} \frac{k T}{m_{G} c^{2}}-\frac{16}{3} \frac{p^{0}}{p_{G}^{0}}\right], \\
\nu= & \widetilde{\eta}_{H S} \frac{c|\mathbf{p}|^{2}}{k T p^{0}}\left[\frac{2}{3}\left(1-\frac{c p^{0}}{k T}\right)\left(\frac{|\mathbf{p}|}{p^{0}}\right)^{2}+\frac{7}{2} \frac{k T}{m_{G} c^{2}} \times\right. \\
& \left.\left(\frac{16}{3}-\left(\frac{p^{0}}{|\mathbf{p}|}\right)^{2}\right)-\frac{7}{2} \frac{p^{0}}{p_{G}^{0}}\right],
\end{aligned}
$$

donde el coeficiente de fricción-viscosidad, para sección transversal constante

$$
\widetilde{\eta}_{H S}=4 \pi n_{G} c \frac{|\mathbf{p}|}{p^{0}} \sigma .
$$

Hay que notar que los coeficientes (4.40)-(4.42) tienen varias contribuciones. Por ejemplo, el tensor de difusión tiene dos términos, uno multiplicado por $\left(\beta^{2} z_{G}\right)^{-1}$, donde recordemos que $\beta \equiv|\mathbf{p}| / p^{0}$. El vector de fricción y la frecuencia característica también tienen un término como éste, pero para estos coeficientes hay otra contribución del orden $p^{0} / p_{G}^{0} \ll 1$ de la cual se puede prescindir debido a la aproximación de gas de Lorentz.

De la ecuación (4.43) vemos que el coeficiente de fricción-viscosidad $\widetilde{\eta}$ no depende de la temperatura, a diferencia del caso anterior del movimiento browniano relativista (4.23), donde $\eta \propto k T$. En este caso, $\widetilde{\eta}$ depende de la velocidad de la partícula del componente que se difunde $|\mathbf{p}| / p^{0}=v / c$. Físicamente esta diferencia puede considerarse razonable. De hecho, en el caso de partículas brownianas no relativistas que se difunden en un gas relativista, la velocidad de las partículas brownianas es aproximadamente igual a cero, en promedio, y por lo tanto no contribuyen a los coeficientes correspondientes que resultan ser proporcionales a la temperatura. En el caso del gas de Lorentz la situación se invierte, de los coeficientes correspondientes se espera que sean proporcionales a la velocidad de las partículas que se difunden, ya que al ser relativistas tienen velocidades altas en comparación con el componente del gas. Este comportamiento marca la diferencia entre las dos situaciones complementarias. Como podemos ver de la expresión (4.43), el coeficiente fricción-viscosidad en el límite ultra relativista es constante y proporcional a la velocidad de la luz. En el régimen no relativista este coeficiente es proporcional a la velocidad de las partículas que se difunden [130].

Ahora se describen brevemente los casos límite no relativista y ultra-relativista de $D^{i j}, A^{i}$ y $\nu$ correspondientes a las expresiones $(4.40),(4.41)$ y $(4.42)$, respectivamente. Notemos que cada coeficiente incluye un par de términos que contienen, un factor de $\left(k T / m_{G} c^{2}\right)\left(p^{0} /|p|\right)^{2}$ y $c|p|^{2} / k T p^{0}$. En ambos casos límite el 
término correspondiente al primer factor es despreciable. En el caso no relativista, esto se debe al hecho de que el factor tiene la forma $\left(k T / m v^{2}\right)\left(m / m_{G}\right) \sim m / m_{G} \ll 1$, donde $k T / m v^{2} \sim k T /\left\langle m v^{2}\right\rangle \sim 1$. Por otra parte, en el caso ultra-relativista $p^{0} /|\mathbf{p}| \sim 1$ y, puesto que el gas de fondo es no-relativista, $k T / m_{G} c^{2} \ll 1$, esto hace que el primer factor sea despreciable también en este límite. El segundo factor en ambos casos adopta la forma del cociente entre la energía correspondiente y energía térmica, $k T$. En este punto, en el caso no relativista tenemos

$$
\begin{aligned}
D_{N R}^{i j} & =\widetilde{\eta}_{N R}\left(p^{i} p^{j}-\frac{|\mathbf{p}|^{2}}{3} \eta^{i j}\right) \\
A_{N R}^{i} & =\widetilde{\eta}_{N R} p^{i}\left(1-\frac{2}{3} \frac{m v^{2}}{k T}\right) \\
\nu_{N R} & =-\frac{2}{3} \widetilde{\eta}_{N R}\left(\frac{m v^{2}}{k T}\right)^{2}
\end{aligned}
$$

donde $\widetilde{\eta}_{N R}=4 \pi n_{G} v \sigma$. Mientras que en el límite ultra relativista son

$$
\begin{aligned}
D_{U R}^{i j} & =\widetilde{\eta}_{U R}\left(p^{i} p^{j}-\frac{|\mathbf{p}|^{2}}{3} \eta^{i j}\right) \\
A_{U R}^{i} & =\widetilde{\eta}_{U R} p^{i}\left(1-\frac{2}{3} \frac{c p^{0}}{k T}\right) \\
\nu_{U R} & =\frac{2}{3} \widetilde{\eta}_{U R} \frac{c p^{0}}{k T}\left(1-\frac{c p^{0}}{k T}\right)
\end{aligned}
$$

donde $\widetilde{\eta}_{U R}=4 \pi n_{G} c \sigma$. Es interesante que en ambos casos límite, el tensor de difusión y el vector de fricción dinámica tienen la misma forma respectivamente, con el único cambio, en el límite no relativista la energía es de $m v^{2}$, mientras que en el relativista tiene la forma $c p^{0}$. Por otro lado, la frecuencia característica $\nu$, se comporta de forma cuadrática en la relación entre las energías cinética y térmica correspondiente, en ambos casos límite (4.46) y (4.49).

\subsection{Ecuación de Fokker-Planck manifiestamente covariante para el gas simple}

Un sistema que también admite una descripción a través de una ecuación de Fokker-Planck es el gas simple relativista que estudiamos en el capítulo 3, pero ahora en una situación no en equilibrio, pero cercana a él. En particular para este sistema puede escribirse una ecuación de Fokker-Planck covariante. Una formulación covariante es ventajosa ya que permite una mejor comprensión y manejo de las cantidades físicas asociadas a los sistemas relativistas.

Para obtener el operador de Fokker-Planck para el caso de un gas simple se puede considerar que la transferencia de momento es pequeño durante las colisiones entre las partículas, como se realizó en las referencias [105] o [129, 136, 137] para el caso no relativista. 
En la referencia [105] el término de colisión relativista, en una versión no manifiestamente covariante, se aproximó por un operador de Fokker-Planck y únicamente se argumentó la forma cómo se podría conseguir una forma covariante. En esta sección seguiremos la metodología de [105] manteniendo la covarianza manifiesta en cada etapa del análisis, es decir, utilizamos vectores de espacio-tiempo en lugar de los vectores espaciales como en [105]. Esto nos permite deducir una generalización covariante relativista de la relación de fluctuación-disipación Einstein entre los coeficientes de fricción y difusión [49].

Consideremos una partícula del gas que se caracteriza por sus coordenadas espacio-tiempo $x_{1}^{\alpha}$ y los componentes de sus 4-momento $p_{1}^{\alpha}$. Como vimos en el capítulo 3 la evolución de $f$ se rige por la ecuación de Boltzmann relativista (4.1) que puede ser reescrita en forma ligeramente distinta

$$
p_{1}^{\alpha} \frac{\partial f_{1}}{\partial x_{1}^{\alpha}}+m_{1} \frac{\partial\left(f_{1} \mathcal{K}^{\alpha}\right)}{\partial p_{1}^{\alpha}}=2 \int\left(f_{2}^{\prime} f_{1}^{\prime}-f_{2} f_{1}\right) \delta\left(p_{2 \mu} p_{2}^{\mu}-m^{2} c^{2}\right) H\left(p_{2}^{0}\right) F \sigma d \Omega d^{4} p_{2},
$$

donde $H\left(p_{2}^{0}\right)$ es la función escalón unitario de Heaviside que se introduce para considerar sólo energías positivas al integrar en $p_{2}^{0}$. La presencia de la función delta de Dirac toma en cuenta la condición de la capa de masa $\left(p_{2}^{0}\right)^{2}-\mathbf{p}_{2}^{2}=m^{2} c^{2}$.

Para aproximar la integral de colisión suponemos que durante la colisión de dos partículas su momento $p_{1}^{\alpha}, p_{2}^{\alpha}$ se modifica únicamente por pequeñas cantidades $\Delta p_{1}^{\alpha}$ y $\Delta p_{2}^{\alpha}$, de tal manera que la función de distribución $f^{\prime}=f\left(p^{\prime \alpha}\right)$ puede desarrollarse alrededor del momento antes de la colisión. Truncando hasta términos de segundo orden en $\Delta p_{1}^{\alpha}$ obtenemos

$$
f\left(p_{1}^{\prime \alpha}\right)=f\left(p_{1}^{\alpha}+\Delta p_{1}^{\alpha}\right)=f\left(p_{1}^{\alpha}\right)+\Delta p_{1}^{\alpha} \frac{\partial f_{1}}{\partial p_{1}^{\alpha}}+\frac{1}{2} \Delta p_{1}^{\alpha} \Delta p_{1}^{\beta} \frac{\partial^{2} f_{1}}{\partial p_{1}^{\alpha} \partial p_{1}^{\beta}},
$$

donde $f_{1}=f\left(p_{1}^{\alpha}\right)$. Antes de insertar (4.51) en el término de colisión (4.50), es útil introducir un nuevo conjunto de variables. Estas son el momento total y el momento relativo de las partículas, que se definen de la siguiente manera

$$
\begin{aligned}
P^{\alpha}=p_{2}^{\alpha}+p_{1}^{\alpha}, & Q^{\alpha}=p_{2}^{\alpha}-p_{1}^{\alpha}, \\
p_{1}^{\alpha}=\frac{1}{2}\left(P^{\alpha}-Q^{\alpha}\right), & p_{2}^{\alpha}=\frac{1}{2}\left(P^{\alpha}+Q^{\alpha}\right) .
\end{aligned}
$$

Además, estas variables satisfacen las siguientes relaciones [12]

$$
P^{\alpha}=P^{\prime \alpha}, \quad P^{\alpha} Q_{\alpha}=P^{\prime \alpha} Q_{\alpha}^{\prime}=0, \quad \text { and } \quad P^{2}=P^{\alpha} P_{\alpha}, \quad Q^{2}=-Q^{\alpha} Q_{\alpha} .
$$

Así, los momentos transferidos $\Delta p_{1}^{\alpha}$ and $\Delta p_{2}^{\alpha}$ pueden escribirse en términos del momento relativo

$$
\Delta p_{1}^{\alpha}=p_{1}^{\prime \alpha}-p_{1}^{\alpha}=-\frac{1}{2} \Delta Q^{\alpha}, \quad \Delta p_{2}^{\alpha}=p_{2}^{\prime \alpha}-p_{2}^{\alpha}=\frac{1}{2} \Delta Q^{\alpha}
$$

Con las ecuaciones (4.51) y (4.55) podemos reescribir el integrando de la integral de colisión en la forma

$$
f_{2}^{\prime} f_{1}^{\prime}-f_{2} f_{1}=\Delta Q^{\alpha} \frac{\partial}{\partial Q^{\alpha}}\left(f_{1} f_{2}\right)+\frac{1}{2} \Delta Q^{\alpha} \Delta Q^{\beta} \frac{\partial}{\partial Q^{\alpha}} \frac{\partial}{\partial Q^{\beta}}\left(f_{1} f_{2}\right),
$$


donde identificamos

$$
\frac{\partial}{\partial Q^{\alpha}}=\frac{1}{2}\left(\frac{\partial}{\partial p_{2}^{\alpha}}-\frac{\partial}{\partial p_{1}^{\alpha}}\right)
$$

Usando estas variables, podemos calcular las integrales sobre los ángulos $d \Omega=\sin \chi d \chi d \varepsilon$, donde en este caso $\chi$ es el ángulo de dispersión. Para realizar los cálculos, elegimos coordenadas tales que las componentes espaciales del momento total son cero, el sistema de referencia donde esto ocurre se conoce como el marco del centro de momento:

$$
P^{\alpha} \rightarrow\left(P^{0}, \mathbf{0}\right), \quad Q^{\alpha} \rightarrow(0, \mathbf{Q})
$$

la segunda relación en (4.58) se sigue de las propiedades (4.54). Necesitamos una expresión para $\Delta Q^{\alpha}$, en este marco de referencia. Asumiendo que $\mathbf{Q}$ esta sobre el eje $x^{3}$, y escribiendo $\mathbf{Q}^{\prime}$ en coordenadas esféricas, obtenemos

$$
Q^{\alpha} \rightarrow Q\left(\begin{array}{l}
0 \\
0 \\
0 \\
1
\end{array}\right), \quad Q^{\prime \alpha} \rightarrow Q\left(\begin{array}{c}
0 \\
\sin \chi \cos \varepsilon \\
\sin \chi \sin \varepsilon \\
\cos \chi
\end{array}\right), \quad \Rightarrow \quad \Delta Q^{\alpha} \rightarrow Q\left(\begin{array}{c}
0 \\
\sin \chi \cos \varepsilon \\
\sin \chi \sin \varepsilon \\
\cos \chi-1
\end{array}\right)
$$

Podemos integrar sobre los ángulos $\chi$ y $\varepsilon$ tal que

$$
\int \Delta Q^{\alpha} d \varepsilon \sigma \sin \chi d \chi=-Q^{\alpha} \Phi
$$

donde

$$
\Phi=2 \pi \int(1-\cos \chi) \sigma \sin \chi d \chi
$$

Notamos que el producto $\Delta Q^{\alpha} \Delta Q^{\beta}$ puede arreglarse como la siguiente matriz

$$
Q^{2}\left(\begin{array}{cccc}
0 & 0 & 0 & 0 \\
0 & \sin ^{2} \chi \cos ^{2} \varepsilon & \sin ^{2} \chi \cos \varepsilon \sin \varepsilon & (\cos \chi-1) \sin \chi \cos \varepsilon \\
0 & \sin ^{2} \chi \cos \varepsilon \sin \varepsilon & \sin ^{2} \chi \sin ^{2} \varepsilon & (\cos \chi-1) \sin \chi \sin \varepsilon \\
0 & (\cos \chi-1) \sin \chi \cos \varepsilon & (\cos \chi-1) \sin \chi \sin \varepsilon & (\cos \chi-1)^{2}
\end{array}\right)
$$

integramos entonces cada componente sobre el ángulo $\varepsilon$ para llegar a lo siguiente

$$
\int \Delta Q^{\alpha} \Delta Q^{\beta} d \varepsilon \sigma \sin \chi d \chi=Q^{2} \int\left(\begin{array}{cccc}
0 & 0 & 0 & 0 \\
0 & \pi \sin ^{2} \chi & 0 & 0 \\
0 & 0 & \pi \sin ^{2} \chi & 0 \\
0 & 0 & 0 & 2 \pi(\cos \chi-1)^{2}
\end{array}\right) \sigma \sin \chi d \chi
$$

A continuación nos limitamos a la aproximación de colisión rasante (como en el caso no relativista [136]), de modo que el ángulo de dispersión es pequeño y por lo tanto se satisface la siguiente aproximación

$$
\cos \chi-1 \simeq-\frac{1}{2} \chi^{2}, \quad \sin ^{2} \chi \simeq 2(1-\cos \chi),
$$


como el término $(\cos \chi-1)^{2}$ es de orden $\chi^{4}$ puede despreciarse. Ahora podemos expresar la matriz del lado derecho de (4.63) en el marco del centro de momento

$$
\left(\begin{array}{llll}
0 & 0 & 0 & 0 \\
0 & 1 & 0 & 0 \\
0 & 0 & 1 & 0 \\
0 & 0 & 0 & 0
\end{array}\right) \rightarrow-\left(\eta^{\alpha \beta}-\frac{P^{\alpha} P^{\beta}}{P^{2}}+\frac{Q^{\alpha} Q^{\beta}}{Q^{2}}\right)
$$

donde $\eta^{\alpha \beta}=\operatorname{diag}\{+,-,-,-\}$ es la métrica plana de Minkowski. De este modo la integral (4.63) se vuelve

$$
\int \Delta Q^{\alpha} \Delta Q^{\beta} d \varepsilon \sigma \sin \chi d \chi=-Q^{2}\left(\eta^{\alpha \beta}-\frac{P^{\alpha} P^{\beta}}{P^{2}}+\frac{Q^{\alpha} Q^{\beta}}{Q^{2}}\right) \Phi,
$$

Las integrales (4.60) y (4.66) pueden relacionarse calculando la divergencia de (4.66), obteniendo el siguien-te resultado

$$
\frac{\partial}{\partial Q^{\alpha}} \int \Delta Q^{\alpha} \Delta Q^{\beta} d \varepsilon F \sigma \sin \chi d \chi=-2 F \Phi Q^{\beta}
$$

Usando la ecuación (4.60) en el lado derecho de (4.67) se puede escribir

$$
\frac{\partial}{\partial Q^{\alpha}} \int \Delta Q^{\alpha} \Delta Q^{\beta} d \varepsilon F \sigma \sin \chi d \chi=2 \int \Delta Q^{\beta} d \varepsilon F \sigma \sin \chi d \chi
$$

Al sustituir (4.68) en la integral de colisión (4.51), podemos factorizar una segunda derivada

$$
\int \frac{\partial}{\partial Q^{\alpha}} \int\left[\frac{\partial}{\partial Q^{\beta}}\left(f_{1} f_{2}\right)\right] \Delta Q^{\alpha} \Delta Q^{\beta} \delta\left(P^{2}-Q^{2}-4 m^{2} c^{2}\right) H\left(P^{0}\right) F \sigma d \Omega d^{4} p_{2} .
$$

Para volver a las variables originales, $p_{1}, p_{2}$, utilizamos (4.57). En estas variables los términos que contienen la derivada de $p_{2}$ desaparecen debido al teorema de la divergencia. Finalmente se tiene la ecuación diferencial de Fokker-Planck manifiestamente covariante que ha resultado de una aproximación en la integral de colisión [49]:

$$
p_{1}^{\alpha} \frac{\partial f_{1}}{\partial x_{1}^{\alpha}}+m \frac{\partial\left(f_{1} \mathcal{K}^{\alpha}\right)}{\partial p_{1}^{\alpha}}=-\frac{\partial}{\partial p_{1}^{\alpha}}\left(f_{1} A^{\alpha}\right)+\frac{\partial^{2}}{\partial p_{1}^{\alpha} \partial p_{1}^{\beta}}\left(f_{1} D^{\alpha \beta}\right),
$$

donde los coeficientes de transporte se definen como

$$
\begin{aligned}
A^{\alpha} & =2 \int f_{2} \Delta p_{1}^{\alpha} \delta\left(p_{2 \mu} p_{2}^{\mu}-m^{2} c^{2}\right) H\left(p_{2}^{0}\right) F \sigma d \Omega d^{4} p_{2}, \\
D^{\alpha \beta} & =\int f_{2} \Delta p_{1}^{\alpha} \Delta p_{1}^{\beta} \delta\left(p_{2 \mu} p_{2}^{\mu}-m^{2} c^{2}\right) H\left(p_{2}^{0}\right) F \sigma d \Omega d^{4} p_{2},
\end{aligned}
$$

donde se hizo uso de (4.55) para expresar $\Delta Q^{\alpha}$ en términos de $\Delta p_{1}^{\alpha} . A^{\alpha}$ se conoce como el vector de fricción dinámica, y $D^{\alpha \beta}$ es el tensor de difusión. Como se adelantó en [105], en el marco del centro momento se tiene $\Delta p_{1}^{0}=0$ y la ecuación (4.70) puede reducirse al caso conocido puramente espacial [105, 138].

La ecuación (4.70) puede tomar la forma de una ecuación de continuidad en el espacio de momentos

$$
p_{1}^{\alpha} \frac{\partial f_{1}}{\partial x_{1}^{\alpha}}=-\frac{\partial}{\partial p_{1}^{\alpha}} \mathcal{S}^{\alpha}
$$


con $\mathcal{S}^{\alpha}$ el flujo de densidad de partículas definido como

$$
\mathcal{S}^{\alpha}=f_{1}\left(A^{\alpha}+m \mathcal{K}^{\alpha}\right)-\frac{\partial}{\partial p_{1}^{\beta}}\left(f_{1} D^{\alpha \beta}\right) .
$$

Si no hay fuerzas externas $\mathcal{K}^{\alpha}=0$ se puede buscar la solución estacionaria y espacialmente homogénea cuando $\mathcal{S}^{\alpha}=0$. Esto conduce a

$$
A^{\alpha}=D^{\alpha \beta} \frac{\partial \ln f}{\partial p_{1}^{\beta}}+\frac{\partial}{\partial p_{1}^{\beta}}\left(D^{\alpha \beta}\right) .
$$

Al igual que en el caso no relativista [136, 137], podemos introducir consideraciones de isotropía para representar los coeficientes de transporte de la siguiente forma

$$
A^{\alpha}=\frac{\mathcal{A}}{m} p^{\alpha}, \quad D^{\alpha \beta}=-\mathcal{D} \eta^{\alpha \beta},
$$

donde $\mathcal{A}$ es el coeficiente de difusión, $m$ es la masa en reposo de las partículas, y $\mathcal{D}$ es el coeficiente de difusión, que está relacionado con la traza del tensor de difusión a través de $\mathcal{D}=-4 D_{\alpha}^{\alpha}$. Al sustituir (4.76) en (4.75) es fácil ver que

$$
\frac{\mathcal{A}}{m} p^{\alpha}=-\mathcal{D} \eta^{\alpha \beta} \frac{\partial \ln f_{1}}{\partial p_{1}^{\beta}}
$$

Para continuar tenemos que realizar la derivada en (4.77) y para ello necesitamos la función de distribución $f_{1}$ para el caso estacionario y homogéneo. Ahora, la condición $\mathcal{S}^{\alpha}=0$ corresponde a la situación de equilibrio, donde el término de colisión desaparece. Por lo tanto en equilibrio, podemos hacer uso de la función de distribución Jüttner que obtuvimos en el capítulo anterior (3.41), que en $d=3$ es

$$
f(p)=\frac{\sqrt{N^{\mu} N_{\mu}}}{2 c(m c)^{3} K_{2}(m c \Theta)}\left(\frac{m c \Theta}{2 \pi}\right) e^{-\Theta_{\alpha} p^{\alpha}},
$$

donde $\Theta_{\alpha}=\frac{u^{\alpha}}{k T}$. Con la sustitución de (4.78) en (4.77) se obtiene que el vector de fricción se puede escribir como

$$
A^{\alpha}=\frac{\mathcal{A}}{m} p^{\alpha}=\mathcal{D} \Theta^{\alpha}=-4 D_{\beta}^{\beta} \Theta^{\alpha} .
$$

Esta es una generalización de la relación fluctuación-disipación de Einstein entre los coeficientes de fricción y difusión $[122,126]$ que en el caso no relativista tiene la forma $\mathcal{D}=k T \mathcal{A}$. Como se muestra en $[49,105]$, esta expresión aparece cuando tomamos el límite no relativista del producto de la ecuación (4.79) por $p_{\alpha}$, es decir, $\mathcal{A}=\frac{\mathcal{D}}{m c^{2}} \Theta^{\alpha} p_{\alpha}$.

\subsection{Discusión}

La necesidad de interpretar ciertos fenómenos astrofísicos y de altas energía de corriente nos conduce naturalmente a investigar más a fondo la incorporación de los principios de la teoría cinética con los de la relatividad. En particular, al estudiar la ecuación de Boltzmann relativista puede resultar útil aproximar el término de colisión por un operador diferencial tipo Fokker-Planck ya que en este se puede estudiar 
el comportamiento de los coeficientes de transporte correspondientes que acompañan a la función de distribución y a sus derivadas.

En este capítulo hemos considerado aproximar el término de colisión de la ecuación de Boltzmann relativista para dos mezclas binarias con condiciones distintas y complementarias. El punto de partida de nuestro análisis es la ecuación de Boltzmann relativista en el que la distribución es una función de momento espacial, utilizando la condición de la capa de masa. Las mezclas binarias se describen por un conjunto de dos ecuaciones de Boltzmann acopladas, una para cada componente.

Dadas las características de los sistemas estudiados, la integral de colisión de la ecuación de Boltzmann correspondiente se redujo, primero a una integral de colisión de la interacción entre partículas de distinta especie. En ambos casos el gas de fondo se considera en equilibrio. En el término de colisión restante se hace un desarrollo de Taylor de la función de distribución, hasta segundo orden, es de ahí que aparece el operador diferencial. Para identificar los coeficientes de transporte es necesario realizar las integrales restantes; es aquí donde cada caso estudiado sigue distintas aproximaciones en relación con el cociente de energías entre los componentes de la mezcla.

El primer caso corresponde al gas de Rayleigh o movimiento Browniano relativista que consiste en una componente diluida formada por partículas pesadas no relativistas difundiéndose en un gas en equilibrio de partículas ligeras que sí son relativistas [105]. Se encontró que la integral de colisión se aproxima por una ecuación de Fokker-Planck cuyo coeficiente es llamado coeficiente de fricción-viscosidad $\eta$ para el cual se obtuvo una expresión en el caso de sección transversal constante. Además se obtuvieron los casos límite ultra relativista y no relativista. En este último el término dominante coincide con lo conocido para el gas de Rayleigh [119, 120].

Es necesario enfatizar que en el gas de Rayleigh relativista analizado aquí, la relajación al equilibrio del sistema está dada por colisiones y no por procesos estocásticos relativistas como en [117, 118]. Como es bien sabido la ecuación de Fokker-Planck en el caso no relativista aparece como una aproximación a la ecuación de Chapman-Kolmogorov que es la ecuación central de los procesos de Markov. Esta aproximación consiste en suponer que la evolución estocástica en Chapman-Kolmogorov se da a través de saltos infinitesimales en un proceso de difusión. Saltos discontinuos o de diferente naturaleza añaden derivadas de órdenes superiores a la ecuación de Fokker-Planck, esto se conoce como expansión de Kramers-Moyal [139, 140]. Podemos preguntarnos si las ecuaciones relativistas en las referencias [117, 118, 141] son únicamente analogías matemáticas de las ecuaciones Fokker-Planck y Langevin. Esto debido a que, además de que su interpretación física es confusa y no se ha llegado a un consenso en la comunidad [118], no parece haber algún principio fundamental detrás de ellas como en el caso de la ecuación de Chapman-Kolmogorov no relativista. La ventaja de nuestro enfoque es que podemos describir la difusión en el sistema a través de aproximaciones físicas sin necesidad de introducir generalizaciones poco claras de los procesos estocásticos. El segundo caso considerado fue un gas de Lorentz relativista, que está formado por dos componentes uno de los cuales está formado por partículas ligeras relativistas que se difunden en un gas en equilibrio que 
se compone de partículas más pesadas y no relativistas cuyo equilibrio se describe por la distribución norelativista de Maxwell-Boltzmann. Este sistema ha sido considerado previamente en la literatura [133, 12]. A diferencia de los casos antes estudiados en este caso llegamos a una ecuación de Fokker-Planck (4.33) que contiene un término sin derivada de la función de distribución. Ese tipo de términos han sido utilizados en la literatura para modelar fuerzas motrices [142], tasas de creación o aniquilación, tasas de crecimiento [143], tasas de reacción [132], y procesos de producción en general. Es común utilizar este tipo de términos para describir efectos de retardo o memoria [144]. En la referencia [145] se estudia un sistema difusivo con un término que codifica la fuerza de la memoria, análogo a nuestro coeficiente $\nu$. Aunque en este trabajo los coeficientes son constantes, el inverso de este coeficiente también corresponde al tiempo de decaimiento del sistema y da lugar a soluciones estacionarias no triviales que dependen de la distribución inicial. Hasta donde sabemos no se conoce hasta la fecha cuál es el papel de dichos términos en los sistemas relativistas y su análisis a fondo se deja como perspectiva.

Los coeficientes (4.30), (4.36) y (4.37) delante de la función de distribución y sus derivadas resultan ser funciones del momento espacial de acuerdo con la literatura en el caso no-relativista [129, 130], así como en el caso relativista [133]. Para continuar el análisis hemos utilizado una sección transversal constante obteniendo coeficientes bastante simples en la que dos contribuciones se pueden distinguir fácilmente, cada uno multiplicado por los factores de $\left(k T / m_{G} c^{2}\right)\left(p^{0} /|p|\right)^{2}$ y $c|p|^{2} / k T p^{0}$, respectivamente, y que son cualitativamente similares a los de [133] para los términos de la energía y el impulso espaciales. Nos dimos cuenta de los primeros factores, es decir, $\left(z_{G} \beta^{2}\right)^{-1}$, son los mismos en la contribución de la energía que [133], y puede ser despreciado en el límites ultra y no relativista. El segundo factor no puede despreciarse y resulta ser, en ambos casos, la relación entre una energía cinética correspondiente y la energía térmica $k T$. Sin embargo, no debemos esperar que estas cantidades coincidan con las calculadas en [133] debido a que la naturaleza de las aproximaciones utilizadas es distinta en ambos tratamientos. Sorprendentemente en nuestro trabajo, así como en [133] aparece un término sin derivada de la función de distribución. Nuestra frecuencia característica $\nu$ delante de dicho término, (4.42), tiene un comportamiento cuadrático en la relación entre las energías cinética y térmica correspondiente, tanto para los casos límite ultra y no relativista. Para el tensor de difusión $D^{i j}$ y el vector de fricción dinámica $A^{i}$ encontramos que estas cantidades tienen la misma forma, en ambos casos ultra-relativista y no relativista, variando sólo la energía correspondiente como se puede ver en (4.44)-(4.45) y (4.47)-(4.48). El comportamiento general de los coeficientes de arriba (4.30), (4.36) y, en particular, $\nu$, (4.37), se deja abierto para investigar en detalle en trabajos posteriores.

Por otra parte, como un trabajo a futuro sería claramente útil para estudiar una versión manifiestamente covariante en el análisis anterior. A menudo es gratificante hacerlo no sólo para comprender mejor las características importantes del sistema relativista, sino de aclarar las cuestiones relacionadas con el cambio de marcos de referencia (Véase, por ejemplo [46, 48]). En esa dirección se mostró como obtener un operador de Fokker-Planck manifiestamente covariante para el gas relativista de una componente en la aproximación 
de colisiones rasantes, cercano al equilibrio. Nuestro punto de partida fue la versión covariante de la ecuación de Boltzmann (4.50), donde se hizo un desarrollo de Taylor del término de colisión considerando pequeña la transferencia de momento, es decir, desviaciones de la distribución manifiestamente covariante (3.41) obtenida en el capítulo 3. Finalmente se pudieron reconocer los coeficientes de transporte como los momentos de la distribución (4.71) y (4.72). Además, este enfoque lleva a una expresión covariante (4.79) que generaliza la relación de fluctuación-disipación de Einstein. 
4.6. Discusión 


\section{Termodinámica estadística de sistemas simples cuantizados por lazos}

La capacidad de la física estadística para relacionar información microscópica de un sistema con su correspondiente descripción macroscópica resulta una idea atractiva para intentar relacionar la posible microestructura del espacio-tiempo con su correspondiente descripción macroscópica que aporta la relatividad general.

Entre las propuestas de teorías cuánticas de la gravedad se tiene una que es independiente del espaciotiempo de fondo: la llamada gravedad cuántica de lazos. En esta propuesta el esquema de cuantización adoptado no es equivalente a la cuantización usual de Schrödinger de la mecánica cuántica. La aplicación de este esquema a sistemas simples con un número finito de grados de libertad se conoce como mecánica cuántica "polimérica"1. Tales modelos capturan algunas características de la cuantización por lazos de la gravedad. Resulta entonces relevante investigar las condiciones bajo las cuales los dos enfoques, el polimérico y el Schrödinger, producen los mismos resultados físicos, en particular para sistemas simples como el oscilador armónico y otros. En este capítulo estudiamos la termodinámica de dos modelos formados por sistemas unidimensionales cuantizados por lazos: un ensamble de osciladores armónicos que puede modelar vibraciones en un cristal o radiación, y un gas ideal de partículas contenido en una caja de volumen fijo.

Las modificaciones "poliméricas" de las cantidades termodinámicas con respecto a las usuales, están relacionadas con una escala de longitud que aparece en la cuantización por lazos. Este parámetro puede interpretarse, por un lado, como una escala fundamental como la longitud de Planck $\left(\ell_{p}=\sqrt{G \hbar / c^{3}} \approx\right.$ $10^{-33} \mathrm{~cm}$ ), o bien, como un regulador para renormalizar el modelo y obtener su límite continuo.

Además de los factores numéricos que dependen de las aproximaciones introducidas, estas modificaciones

\footnotetext{
${ }^{1}$ Es conviene aclarar que los lazos de este esquema de gravedad cuántica, corresponden a gráficos encajados en el espacio (esto se precisará más adelante). Estos proveen un espectro discreto para los operadores geométricos como el de área y el de volumen. Estos gráficos asemejan estructuras poliméricas, de ahí el uso de tal vocablo.
} 
se pueden identificar con otras obtenidas en la literatura bajo la suposición de la existencia de una longitud mínima y el correspondiente principio de incertidumbre generalizado. Mostramos que esta coincidencia se puede explicar a la luz de la versión en la cuantización por lazos de la relación de incertidumbre. Nuestros modelos indican que este resultado para el análisis de muchos cuerpos de un sistema simple polimérico cuántico puede ser genérico.

\subsection{Introducción}

El principal objetivo de la física estadística es obtener información macroscópica de un sistema a partir de la dinámica de sus componentes microscópicos. Como hemos visto en los capítulos anteriores estos métodos pueden extenderse al régimen relativista y a muchos otros sistemas [146]. En vista de ello resulta atractiva la idea de intentar relacionar la posible micro-estructura del espacio-tiempo que surge en propuestas de cuantización de la gravedad, con su correspondiente descripción macroscópica que aporta la teoría de la relatividad general. Un ejemplo de ello, donde se utilizan métodos de la física estadística es la llamada gravedad estocástica [42]. En este enfoque se generaliza la ecuación de Einstein incluyendo un término estocástico que modela las fluctuaciones cuánticas del espacio-tiempo. Este término induce desviaciones a las geodésicas clásicas de partículas que se propagan en el espacio-tiempo. Es decir, este enfoque hace una analogía con las ecuaciones estocásticas de Langevin [126] que gobiernan el movimiento Browniano ${ }^{2}$. Uno de los propósitos más importantes de la gravedad cuántica es investigar la descripción de la estructura del espacio-tiempo a la escala de Planck $\left(\ell_{p}=\sqrt{G \hbar / c^{3}} \approx 10^{-33} \mathrm{~cm}\right)$. Entre las propuestas para cuantizar la gravitación se encuentra la llamada gravedad cuántica de lazos [147, 148], la cual surge al combinar la mecánica cuántica y la relatividad general en un esquema canónico independiente del espacio-tiempo de fondo ${ }^{3}$. Un resultado crucial de esta propuesta es que los operadores cuánticos de entes geométricos como la longitud, el área y el volumen tienen un espectro discreto.

El principal éxito de este enfoque ha sido el evitar la singularidad clásica asociada con el Big Bang que surge en un tratamiento clásico basado en la Relatividad General. En lugar de la singularidad clásica se obtiene un rebote cuántico o Big Bounce por donde la dinámica continúa [149, 150, 151, 152]. Hay también algunos avances en la descripción cuántica de hoyos negros, se ha calculado la entropía de un agujero negro de Schwarzschild a partir de bases microscópicas recuperando la fórmula de BekensteinHawking [153, 154, 155].

En ambos casos la termodinámica tanto en equilibrio como fuera de éste, juega un papel clave en la descripción de dichos sistemas, por ejemplo al estudiar el proceso final de evaporación de agujeros negros [39]. Concretamente en el estudio de la historia térmica del universo, una de las etapas más interesantes de los inicios del universo son los orígenes de los fondos de partículas primordiales [28], [67]. Como es bien

\footnotetext{
${ }^{2}$ Como se vió en el capítulo 4 hay alternativas al uso de ecuaciones estocásticas en la descripción del movimiento Browniano, en particular en su versión relativista.

${ }^{3}$ Para una revisión sobre este tema ver el apéndice E.
} 
conocido en la cosmología inflacionaria, las perturbaciones de densidad y las ondas gravitacionales son consistentes con las observaciones del Fondo Cósmico de Microondas (CMB) y podría explicar el origen de la estructura en el universo [156]. Actualmente es de gran interés el estudio de los remanentes estocásticos de gravitones y sus propiedades estadísticas [157], [158]. De hecho, en la cosmología cuántica de lazos, la etapa inflacionaria puede aparecer como un efecto de la geometría cuántica [159], [160]. Además, se han calculado diversas contribuciones al espectro de gravitones producido durante el período superinflacionario en el esquema de la cosmología de lazos [161], [162], [163], [164].

Claramente es de interés comprender la descripción termodinámica de los sistemas cuantizados en forma polimérica o por lazos, en particular los sistemas simples pueden ser más fáciles de explorar. La cuantización por lazos aplicada a un sistema con un número finito de grados de libertad se denomina mecánica cuántica "polimérica". El término "polimérico" se utiliza debido a que en la gravitación cuántica de lazos, los estados cuánticos de la gravedad se representan por gráficos encajados en el espacio, estos gráficos son muy similares a las complejas estructuras de las macromoléculas poliméricas. Este modelo ha sido útil ya que ilustra algunas características matemáticas y conceptuales que se plantean en la gravedad cuántica de lazos [165]-[168], [169], particularmente tiene el mismo espacio de configuración que el de la cosmología cuántica de lazos [170], por lo que su análisis podría ser de utilidad en el caso cosmológico. Se han estudiado además simetrías espaciales, lo que ha llevado a un conflicto con el álgebra de Galileo, a diferencia de lo que ocurre en la mecánica cuántica de Schrödinger [171]. Este problema aún requiere más trabajo para ser aclarado.

En la mecánica cuántica polimérica o de lazos, aparece un parámetro que juega el papel de una escala de longitud característica. Hay al menos dos posibles interpretaciones para este parámetro en la cuantización por lazos. Por un lado se le puede identificar con una longitud fundamental como la longitud de Planck $\left(\ell_{p}=\sqrt{G \hbar / c^{3}} \approx 10^{-33} \mathrm{~cm}\right)$, en este caso es de esperarse que los efectos resultantes sean diminutos. Alternativamente se ha considerado a tal parámetro como una escala reguladora que permite realizar un procedimiento de renormalización al modelo; su límite continuo resulta ser la mecánica cuántica de Schrödinger [166], [167]. Sin embargo, las investigaciones realizadas hasta el momento no son concluyentes, ya que sólo se analiza un sistema que tiene un flujo de renormalización trivial ${ }^{4}$. Por lo tanto la cuestión de si la cuantización por lazos es equivalente a la usual en el límite continuo requiere mayor investigación. Esta cuantización también se ha utilizado para explorar la evasión de la singularidad clásica de los potenciales tales como el potencial de Coulomb $1 / r$ [168], y 1/ $r^{2}$ [172]; mostrando que la cuantización polimérica de este tipo de potenciales singulares, puede producir una teoría física bien definida, sin cambiar el potencial cerca de la singularidad. Esto abre la posibilidad de tomar en serio la cuantización por lazos como un método de cuantización que, más allá de sólo reproducir algunos efectos matemáticos de la cuantización por lazos, también puede resolver algunos problemas en sistemas cuánticos comunes.

Resulta interesante que aún cantidades termodinámicas obtenidas desde un esquema estadístico, son

\footnotetext{
${ }^{4}$ En teoría cuántica de campo, el flujo trivial está dado por la variación de escala de las constantes de acoplamiento
} 
sensibles a este esquema de cuantización.

En este capítulo se considera, en primer término, la descripción termodinámica de sistemas simples ${ }^{5}$. Primero estudiamos los eigenvalores de energía para el oscilador armónico [165], y una partícula confinada en una caja con volumen finito. Para obtener cantidades termodinámicas como en mecánica estadística estándar [81], calculamos la correspondiente función de partición canónica a partir de los espectros de energía anteriores. Las cantidades que hemos encontrado tienen correcciones que son función de la escala característica de la cuantización polimérica. Es importante aclarar que la discretez inducida por la escala es la única fuente de correcciones ya que no están presentes los efectos gravitatorios. Con este modelo sencillo tratamos de dilucidar el papel que dicha cuantización no estándar puede jugar en un estudio estadístico de sistemas de muchos cuerpos.

Las cantidades termodinámicas modificadas por la cuantización de lazos que encontramos se asemejan a aquellas obtenidas en el contexto de la fenomenología de la gravedad cuántica [173], [174]. Este enfoque surge cuando se consideran algunos aspectos genéricos que aparecen en común entre las propuestas de la gravedad cuántica, como una escala de longitud mínima y posibles violaciones a la simetría de Lorentz. Estos aspectos se reflejan en una modificación de la relación de dispersión (MDR) entre la energía y el momento [175]-[178], y en una generalización de la relación de incertidumbre (GUP) [179]-[182]. Tales efectos gravitacionales cuánticos son importantes, ya que son susceptibles de constreñirse experimentalmente ${ }^{6}$.

\subsection{Representación por lazos de la Mecánica Cuántica}

En esta sección describimos la cuantización por lazos de un sistema simple que consiste en una partícula no relativista en movimiento en un subconjunto de la recta real $\mathbb{R}$ llamado gráfico. Este modelo fue introducido por Ashtekar, Fairhurst y Willis [165]. Si bien el modelo mimetiza la estructura discreta subyacente del espacio, no captura cuestiones como el problema de las constricciones de la relatividad general y no predice una estructura del espacio, sino que la supone ${ }^{7}$.

En la formulación algebraica de la mecánica cuántica usual un estado está representado por un vector en un espacio de Hilbert $\mathcal{H}$. Se definen los operadores auto-adjuntos $\hat{O}$ actuando sobre $\mathcal{H}$, de tal forma que sus valores esperados son los observables o cantidades medibles en el experimento. La evolución está dada por una familia de de transformaciones unitarias en $\mathcal{H}$ generadas por el operador Hamiltoniano. La guía básica para construir una teoría cuántica a partir de un sistema que sigue las reglas clásicas es la relación entre el paréntesis de Poisson clásico y el conmutador que define el álgebra de operadores

$$
\left[\hat{O}_{1}, \hat{O}_{2}\right]=-i\left\{\widehat{O_{1}, O_{2}}\right\}
$$

\footnotetext{
${ }^{5}$ Cabe mencionar que sistemas como el universo temprano y los agujeros negros, son sistemas fuera del equilibrio por lo que el análisis debería incluir los correspondientes procesos de transporte. Esto podría tratarse a través del esquema proporcionado por la teoría cinética [129], [25].

${ }^{6}$ De hecho, los límites actuales a violaciones de la invariancia de Lorentz son bastante estrictos [174, 175].

${ }^{7}$ Ver apéndice E para revisión conceptual de este tema.
} 
con $\hbar=1$ por el momento. De esta forma la cuantización consiste en reemplazar las variables canónicas clásicas $q, p$ de un sistema, por operadores auto-adjuntos que cumplen con las relaciones de conmutación canónicas:

$$
\begin{gathered}
{[\hat{q}, \hat{q}]=[\hat{p}, \hat{p}]=0,} \\
{[\hat{q}, \hat{p}]=\hat{q} \hat{p}-\hat{p} \hat{q}=i \hat{I},}
\end{gathered}
$$

donde $\hat{I}$ es la identidad sobre el espacio de Hilbert $\mathcal{H}$. Estas relaciones de conmutación son las expresiones que definen el álgebra de Heisenberg. A partir de las expresiones (5.2) y (5.3) pueden definirse los operadores acotados

$$
\hat{U}(\lambda)=e^{i \lambda \hat{q}}, \quad \hat{V}(\mu)=e^{i \mu \hat{p}}
$$

Se dice que (5.4) son operadores unitarios de un parámetro con generadores $\hat{q}$ y $\hat{p}$ respectivamente [183]. Dada la relación de conmutación canónica (5.3) se puede ver, aplicando la fórmula de Hausdorff-BakerCampbell, que los operadores $\hat{U}$ y $\hat{V}$ satisfacen la siguiente relación conocida como la relación de Weyl

$$
\hat{U}(\lambda) \hat{V}(\mu)=e^{-i \lambda \mu} \hat{V}(\mu) \hat{U}(\lambda)
$$

Al conjunto de operadores $\hat{U}$ y $\hat{V}$, junto con $\mathcal{H}$ se le llama la representación del álgebra. A la representación usual de la mecánica cuántica donde $\mathcal{H}=L^{2}(\mathbb{R})$ y los generadores son $\hat{p}$ actuando por derivación y $\hat{x}$ actuando por multiplicación, se le llama representación de Schrödinger.

En la cuantización por lazos se construye el espacio de Hilbert cinemático $\mathcal{H}_{\text {poly }}$, considerando un subconjunto $\gamma$ de la recta real llamado gráfico. El gráfico $\gamma$ es una colección numerable de puntos $\gamma=\left\{x_{j}\right\}_{j \in I} \subset \mathbb{R}$ sujeto a condiciones técnicas que garantizan la convergencia de ciertas series (los detalles pueden consultarse en [165]). Se define el espacio de funciones cilíndricas $\mathrm{Cyl}_{\gamma}$ respecto a algún gráfico $\gamma$, como el conjunto de las funciones complejas univaluadas del tipo:

$$
\mathrm{Cyl}_{\gamma}:=\left\{f(k): f(k)=\sum_{j} f_{j} e^{-i x_{j} \cdot k} ; f_{j} \in \mathbb{C}, \sum_{j}\left|f_{j}\right|^{2}<\infty\right\},
$$

donde $f_{j}$ son funciones complejas bien comportadas. Además se introduce el espacio de funciones que son cilíndricas respecto de algún gráfico simplemente como $\mathrm{Cyl}:=\bigcup_{\gamma} \mathrm{Cyl}_{\gamma}$. Finalmente el espacio de Hilbert cinemático polimérico es la cerradura de Cauchy de Cyl respecto a un producto interno definido utilizando la medida de Haar:

$$
\left\langle x_{i} \mid x_{j}\right\rangle=\lim _{T \rightarrow \infty} \frac{1}{T} \int_{a}^{a+T} d k e^{i k\left(x_{i}-x_{j}\right)}=\delta_{x_{i}, x_{j}},
$$

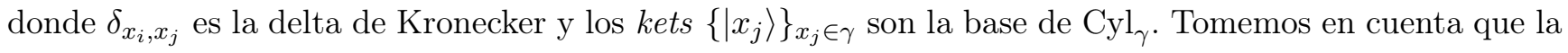
base de los estados en $\mathcal{H}_{\text {poly }},\left|x_{j}\right\rangle$ es no numerable, están etiquetados por números arbitrarios $x_{j} \in \mathbb{R}$. Este espacio de Hilbert se puede expresar como $\mathcal{H}_{\text {poly }}=L^{2}\left(\tilde{\mathbb{R}}_{B o h r}, d \mu_{\text {Bohr }}\right)$, donde $\tilde{\mathbb{R}}_{B o h r}$ es $\mathbb{R}$ con topología discreta y es el dual algebraico de la llamada compactificación de Bohr, $\mathbb{R}_{\text {Bohr }}$, de la recta real $\mathbb{R} ;$ mientras $d \mu_{\text {Bohr }}$ es la medida de Haar definida en (5.7). 
En esta cuantización se escoge usar el álgebra de Weyl, los operadores básicos utilizados en esta cuantización son $\hat{U}(\lambda)$ y $\hat{V}(\mu)$ y su acción sobre un estado $\left|x_{j}\right\rangle$ de la base ortonormal es:

$$
\hat{U}(\lambda)\left|x_{i}\right\rangle=e^{i \lambda x_{i}}\left|x_{i}\right\rangle, \quad \hat{V}(\mu)\left|x_{i}\right\rangle=\left|x_{i}-\mu\right\rangle .
$$

Es importante notar que aunque el operador $\hat{V}(\mu)$ provee un grupo unitario uniparamétrico en $\mathcal{H}_{\text {poly }}$, este no es débilmente continuo en el parámetro $\mu$. Esto se sigue del hecho de que no importa que tan pequeño sea $\mu$ dos estados $\left|x_{i}\right\rangle$ y $\hat{V}(\mu)\left|x_{i}\right\rangle$ siempre serán ortogonales, esto se debe al producto interno que se introdujo en (5.7). Por un lado se tiene que

$$
\lim _{\mu \rightarrow 0}\left\langle x_{i}|\hat{V}(\mu)| x_{i}\right\rangle=0,
$$

mientras que $\hat{V}(\mu=0)=\hat{I}$ y $\left\langle x_{i} \mid x_{i}\right\rangle=1$. De esta forma no hay un operador auto-adjunto $\hat{p}$ en $\mathcal{H}_{\text {poly }}$ que sea un generador infinitesimal de $\hat{V}(\mu)$ tal que pueda expresarse como en (5.4). En este caso de la cuantización por lazos, el teorema de Stone-von Neumann [39] ya no es aplicable ${ }^{8}$ el cual asegura que cualquier representación irreducible y fuertemente continua del álgebra de Weyl es equivalente a la representación de Schrödinger usual donde el espacio de Hilbert es $L^{2}(\mathbb{R})$, el espacio de las funciones de cuadrado integrable en $\mathbb{R}$. Al no ser aplicable la representación polimérica no es equivalente a la de Schrödinger ${ }^{9}$.

Utilizaremos entonces el álgebra Weyl-Heisenberg con la posición $\hat{x}$ y el operador desplazamiento $\hat{V}(\mu)$ en lugar de el operador de momento; la relación de conmutación que siguen estos operadores es la siguiente:

$$
[\hat{x}, \hat{V}(\mu)]=-\mu \hat{V}(\mu) .
$$

Dado que no hay un operador de momento bien definido, cualquier función en el espacio de fase que dependa del momento necesita ser regularizado. Sea $\mu_{0}>0$ una escala elegida a conveniencia y que puede ser considerada como una escala fundamental. Una de las opciones más sencillas consiste en definir un operador análogo al operador de momento de la siguiente manera:

$$
\hat{K}_{\mu_{0}}=\frac{1}{2 i \mu_{0}}\left[\hat{V}\left(\mu_{0}\right)-\hat{V}\left(-\mu_{0}\right)\right] .
$$

Esta opción se sugiere debido a que, en el caso de la mecánica cuántica de Schrödinger, se puede hacer un desarrollo del operador de translación (5.4), para $\mu$ pequeña tal que a primer orden

$$
\hat{p}=\frac{e^{i \mu \hat{p}}-e^{-i \mu \hat{p}}}{2 i \mu}-\mathcal{O}\left(\hat{p}^{2}\right) .
$$

\footnotetext{
${ }^{8}$ Por completez el teorema de Stone-von Neumann se enuncia de la siguiente manera:

Teorema 1 (Stone-von Neumann). Para un sistema con un número finito de grados de libertad sean $(\hat{U}, \hat{V} ; \mathcal{H})$ y $\left(\hat{U}^{\prime}, \hat{V}^{\prime} ;\right.$ $\left.\mathcal{H}^{\prime}\right)$ dos conjuntos irreducibles de operadores unitarios fuertemente continuos que cumplen el álgebra de Weyl. Entonces $(\hat{U}$, $\hat{V} ; \mathcal{H})$ y $\left(\hat{U}^{\prime}, \hat{V}^{\prime} ; \mathcal{H}^{\prime}\right)$ son unitariamente equivalentes.

${ }^{9}$ En el caso de la cosmología cuántica de lazos hay argumentos sobre cuales representaciones son correctas en el sentido de que reproducen resultados conocidos [184].
} 
La dinámica estará dada por un Hamiltoniano expresado en términos del operador de traslación $\hat{V}(\mu)$

$$
\widehat{H}_{\mu_{0}}=\frac{\hbar}{2 m} \widehat{K_{\mu_{0}}^{2}}+\widehat{W}(x)
$$

donde $\widehat{W}(x)$ es un potencial que sólo depende de $x$, y el término cinético está definido ${ }^{10}$ como [165]:

$$
\widehat{K_{\mu_{0}}^{2}}=\frac{1}{4 \mu_{0}^{2}}\left(2-\hat{V}\left(2 \mu_{0}\right)-\hat{V}\left(-2 \mu_{0}\right)\right) \text {. }
$$

El espacio de Hilbert $\mathcal{H}_{\text {poly }}$ puede descomponerse en subespacios superselectos de dimensión finita. Dada la escala $\mu_{0}$, la acción del Hamiltoniano (5.13) hace que cada subespacio superselecto esté completamente definido en una gráfica regular $=\gamma\left(\mu_{0}, x_{0}\right)=\left\{x_{0}+\mu_{0} j\right\}$ con $j \in \mathbb{Z}$ y $x_{0} \in\left[0, \mu_{0}\right)$, donde $\mu_{0}$ es el tamaño del paso de la red regular y $x_{0}$ el punto de referencia (Fig. 5.1).

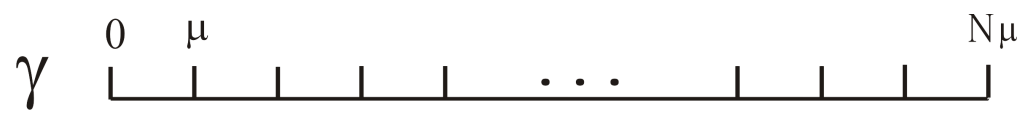

Figura 5.1: Red regular $\gamma$ de tamaño $\mu$.

Los subespacios son etiquetados por el punto de referencia $x_{0}$ y el espacio de Hilbert polimérico se descompone como una suma directa de espacios de Hilbert separables superselectos $\mathcal{H}_{x_{0}}$

$$
\mathcal{H}_{\text {poly }}=\bigoplus_{x_{0} \in\left[0, \mu_{0}\right)} \mathcal{H}_{x_{0}}
$$

Por lo tanto, la evolución temporal puede describirse en espacios de Hilbert separables asociados a las gráficas regulares. Limitarse a un gráfico, es matemáticamente equivalente a resolver la versión discreta de la ecuación de Schrödinger convencional en una red regular. Considerando una posible regularización del Hamiltoniano como (5.13), la ecuación de Schrödinger podría escribirse como

$$
i \hbar \frac{d}{d t}|\psi(t)\rangle=\widehat{H}_{\mu_{0}}|\psi(t)\rangle
$$

donde el parámetro temporal se elige como una variable continua.

Como veremos la relación de incertidumbre polimérica tendrá un papel importante en la interpretación de nuestros resultados. En [165] fue examinada en detalle esta relación sin embargo, es necesario comentar algunos aspectos relevantes en su formulación. Se dice que un estado $\left(\Psi \mid \in \mathrm{Cyl}^{*}\right.$ es un estado semiclásico con respecto a cierto conjunto de observables y centrado en un punto $(q, p)$ en el espacio fase clásico, si los valores de expectación de cualquier conjunto de observables, por ejemplo $\hat{A}$, es igual a su valor clásico $A(x, p)$, dentro de ciertas tolerancias especificadas $\tau_{A}$, de modo que las fluctuaciones son pequeñas [165]

$$
\begin{aligned}
\frac{\left(\Psi|\hat{A}| \Psi_{\gamma}^{s}\right\rangle}{\left\|\Psi_{\gamma}^{s}\right\|^{2}} & =A(x, p)\left(1+\tau_{A}^{(1)}\right), \\
\frac{\left(\Psi\left|\hat{A}^{2}\right| \Psi_{\gamma}^{s}\right\rangle}{\left\|\Psi_{\gamma}^{s}\right\|^{2}}-\left(\frac{\left(\Psi|\hat{A}| \Psi_{\gamma}^{s}\right\rangle}{\left\|\Psi_{\gamma}^{s}\right\|^{2}}\right)^{2} & \leq \tau_{A}^{(2)},
\end{aligned}
$$

\footnotetext{
${ }^{10}$ Como el Hamiltoniano es el operador que da la dinámica del sistema, usualmente se cambia $\mu_{0} \rightarrow 2 \mu_{0}$.
} 
para gráficos $\gamma$ suficientemente refinados. El estado $\left.\mid \Psi_{\gamma}^{s}\right)$ es la proyección en $\mathrm{Cyl}_{\gamma}$ de $\left(\Psi \mid \in \mathrm{Cyl}_{\text {y se conoce }}\right.$ como estado sombra [165]. Se puede comprobar que las sombras de los estados coherentes poliméricos [165] saturan la desigualdad de Schwartz, de la que se desprende una relación de incertidumbre, que para dos observables físicos relevantes $A$ y $B$ es

$$
(\Delta A)^{2}(\Delta B)^{2} \geq \frac{1}{4}\|\langle[\hat{A}, \hat{B}]\rangle\|^{2}
$$

Entonces, para obtener la relación de incertidumbre correspondiente, primero tenemos que calcular el conmutador entre los operadores $\hat{x}$ y $\hat{K}_{\mu_{0}}$. Utilizando (5.10) y (5.11) obtenemos directamente que

$$
\left[\hat{x}, \hat{K}_{\mu_{0}}\right]=\frac{i}{2}\left(\hat{V}\left(\mu_{0}\right)+\hat{V}\left(-\mu_{0}\right)\right)=i\left(1-\frac{1}{2} \mu_{0}^{2} \widehat{K_{\mu_{0}}^{2}}\right)
$$

en términos del operador $\widehat{K_{\mu_{0}}^{2}}$. En (5.20) podemos notar que el conmutador entre $\hat{x}$ y $\hat{K}_{\mu_{0}}$ no es simplemente proporcional a la identidad, sino que tiene correcciones de orden $\widehat{K_{\mu_{0}}^{2}}$, y puede escribirse en términos del operador $\hat{V}$.

En contraste a los enfoques fenomenológicos, con este modelo de cuantización por lazos tenemos una representación cerrada del conmutador canónico en lugar de únicamente aproximaciones como ocurre en las generalizaciones de la relación de incertidumbre, (GUP). Para obtener el resultado buscado calculamos la norma del valor de expectación del conmutador utilizando las sombras de los estados coherentes poliméricos

$$
(\Delta x)^{2}\left(\Delta K_{\mu_{0}}\right)^{2}=\frac{1}{2} e^{-\frac{\mu_{0}^{2}}{2 d^{2}}}\left(1+\cos 2 \mu_{0} k\right) \approx 1-\frac{\mu_{0}^{2}}{2 d^{2}}-\mu_{0}^{2} k^{2}+\mathcal{O}\left(\frac{\mu_{0}^{4}}{d^{2}} k^{2}\right)
$$

donde $d$ es un parámetro con dimensiones de longitud que caracteriza al estado semiclásico y que toma valores específicos dependiendo del sistema que se considere. Por ejemplo, en el caso del oscilador armónico $d=\sqrt{\hbar / m \omega}$.

La ecuación (5.21) nos dice que hay al menos, dos condiciones que deben ser satisfechas por la escala $\mu_{0}$, primero $\mu_{0} \ll d$, y $\mu_{0} \ll k^{-1}$. Notemos que a primer orden en $\mu_{0}$ recuperamos la relación de incertidumbre usual identificando $\hat{p} \rightarrow \hbar \hat{K}_{\mu_{0}}$. La expresión obtenida (5.21) puede ser comparada con el principio de incertidumbre generalizado [185]

$$
\Delta x \Delta p=\frac{\hbar}{2}\left(1+\frac{\ell_{\min }^{2}}{\hbar^{2}} \Delta p^{2}+\ldots\right)
$$

donde $\ell_{\text {min }}$ es una escala de longitud mínima y $\Delta p$ es el correspondiente rango de momento. Observemos que el término de modificación en (5.22) es similar al segundo término de corrección en (5.21). De (5.21) vemos que la relación de incertidumbre polimérica es más compleja que (5.22) debido a que hay dos tipos de condiciones sobre la escala, como consecuencia del tipo de cuantización. 


\subsection{Eigenvalores del Hamiltoniano Polimérico}

De la ecuación (5.16) nos damos cuenta que la ecuación de eigenvalores correspondiente a resolver será

$$
\widehat{H}_{\mu_{0}}|\psi\rangle=E|\psi\rangle,
$$

En el caso del Hamiltoniano regularizado (5.13) en una red regular $\gamma\left(x_{0}, \mu_{0}\right)$, cualquier estado $|\psi\rangle \in \mathcal{H}_{\text {poly }}$ es de la forma

$$
|\psi\rangle=\sum_{j \in \mathbb{Z}} \psi\left(x_{0}+j \mu_{0}\right)\left|x_{0}+j \mu_{0}\right\rangle .
$$

Al substituir $|\psi\rangle$ de (5.24) en (5.23) obtenemos una ecuación en diferencias en la representación de posiciones

$$
\frac{\hbar^{2}}{2 m \mu_{0}^{2}}\left[2 \psi\left(x_{j}\right)-\psi\left(x_{j}+\mu_{0}\right)-\psi\left(x_{j}-\mu_{0}\right)\right]=\left[E-W\left(x_{j}\right)\right] \psi\left(x_{j}\right),
$$

donde $x_{j}=x_{0}+j \mu_{0}$. Utilizando la transformada de Fourier discreta definida como

$$
\psi(k)=\left(k|\psi\rangle=\sum_{j \in \mathbb{Z}} \psi\left(x_{0}+j \mu_{0}\right) e^{-i k\left(x_{0}+j \mu_{0}\right)},\right.
$$

con $k \in\left[-\pi / \mu_{0}, \pi / \mu_{0}\right]$, y la función $\psi(k)$ que satisface la condición

$$
\psi\left(\frac{\pi}{\mu_{0}}\right)=e^{-2 \pi i \frac{x_{0}}{\mu_{0}}} \psi\left(-\frac{\pi}{\mu_{0}}\right),
$$

la ecuación (5.25) se puede escribir de la siguiente manera:

$$
\left(1-\frac{\mu_{0}^{2} m}{\hbar^{2}} E-\cos k \mu_{0}\right) \psi(k)=-\frac{\mu_{0}^{2} m}{\hbar^{2}} \sum_{j \in \mathbb{Z}} \psi\left(x_{0}+j \mu_{0}\right) W\left(x_{0}+j \mu_{0}\right) e^{-i k\left(x_{0}+j \mu_{0}\right)} .
$$

En general, se analiza el potencial $W(x)$ y se reescribe el factor $\mu_{0}^{2} m / \hbar^{2}$ en términos de alguna longitud característica del sistema $d$ para así obtener el espectro correspondiente. En el análisis siguiente nosotros consideramos dos casos específicos: el oscilador armónico y una partícula en una caja.

\subsubsection{Oscilador Armónico}

El oscilador armónico polimérico o cuantizado por lazos se había estudiado en [165], utilizando el potencial $W(x)=\hbar \omega x^{2} / 2 d^{2}$ en (5.28) reemplazando la masa en la ecuación de Schrödinger con la longitud $d^{2}=$ $\hbar / m \omega$ que es característica del oscilador. Después de realizar una transformada de Fourier al lado derecho de (5.28) para este potencial se obtiene una ecuación diferencial de segundo orden que puede reconocerse como una ecuación de Mathieu. Como es bien sabido [68], hay una infinidad de soluciones cuasiperiódicas de esta ecuación. Además, la ecuación de Mathieu tiene eigenvalores discretos en un desarrollo asintótico del cual puede identificarse el siguiente espectro de energías

$$
E_{n}=\left(n+\frac{1}{2}\right) \hbar \omega-\left(\frac{2 n^{2}+2 n+1}{32}\right) \lambda^{2} \hbar \omega+\mathcal{O}\left(\lambda^{4}\right),
$$


donde introducimos $\lambda=\mu_{0} / d$ como un parámetro de longitud adimensional, con $\mu_{0} \ll d$. Los términos en (5.29) que modifican el espectro usual del oscilador en el esquema de Schrödinger, disminuyen la energía por un factor del orden de $\mathcal{O}\left(\lambda^{2}\right)$.

De (5.29) podemos ver que el espectro no está acotado por abajo. Esto es un indicio de que considerar este espectro como el correcto no tiene sentido físico a este orden en $\lambda$, es probable que a ordenes más altos el espectro polimérico tenga una comportamiento distinto. En esta aproximación estamos considerando únicamente el primer término en un desarrollo de la forma

$$
E_{n}=E_{n}^{(0)}+\Delta^{(1)} E_{n}(\lambda)+\Delta^{(2)} E_{n}(\lambda)+\ldots
$$

aquí $E_{n}^{(0)}$ es el espectro conocido en la cuantización de Schrödinger. Para garantizar que este espectro no tenga energía infinitamente negativa, pedimos que el primer término sea mucho mayor que la primera corrección, esto nos da una condición sobre el valor que el número cuántico $n$ puede tomar para que la aproximación sea válida, es decir, existe un $n_{\max }$ que depende de $\lambda$

$$
n_{\max } \approx 16 \lambda^{-2}
$$

Esto significa que no podemos explorar el espectro con valores de $n$ mayores que la cota impuesta en (5.31). En [165] se estimó este valor para una longitud mínima de $\mu_{0}=10^{-19} \mathrm{~m}$ que corresponde a la máxima energía que puede ser alcanzada experimentalmente, y con $d=10^{-12} m$ característica de la molécula de monóxido de carbono, lo cual nos da $\lambda=10^{-7}$, con lo que podemos calcular $n_{\max }$, y así los eigenvalores que corresponden a valores mayores de $n_{\max } \simeq 10^{14}$ no tienen realidad física. Esto podría tener problemas con el límite clásico $n \rightarrow \infty$. No obstante, una posibilidad es que en el límite $\mu_{0} \rightarrow 0$ se recupera $n_{\max } \rightarrow \infty$. Otra opción es que, como se muestra en [166] antes de llegar al límite clásico, es posible que sea necesario implementar el límite continuo en el que la cuantización por lazos que aproximará a la mecánica cuántica de Schrödinger. Alternativamente en [166] se introduce una energía de corte (cut-off) en los eigenvalores de energía, que depende de la escala que se utiliza en la regularización. Este esquema es natural en el sentido de que el péndulo considerado en [166], es un sistema que se tiene a la mano y que se sabe se aproxima así por el oscilador armónico. El problema de la introducción de una escala de corte para los sistemas cuantizados por lazos se abordará más adelante.

\subsubsection{Partícula en una caja}

El siguiente ejemplo consiste en una partícula confinada en una caja de tamaño $L=N \mu_{0}$. En este caso, en lugar de trabajar en el espacio de Fourier, podemos utilizar directamente la ecuación en diferencias (5.25). De la misma forma que en el caso usual, el potencial puede definirse como

$$
W\left(x_{j}\right)= \begin{cases}0, & x_{0}<x_{j}<x_{0}+L \\ \infty, & \text { de otro modo }\end{cases}
$$


donde $x_{0} \in\left[0, \mu_{0}\right)$. A través de estas condiciones de frontera este potencial puede extenderse sobre todo $\mathcal{H}_{\text {poly }}$. La función de onda sólo puede entenderse dentro de una región donde la partícula está confinada, fuera de la cual es igual a cero. Dentro de esta región la partícula se comporta como una partícula libre. Una versión de la partícula libre polimérica fue estudiada en [167].

$$
\psi\left(x_{0}\right)=\psi\left(L+x_{0}\right)=0, \forall x_{0} \in\left[0, \mu_{0}\right) .
$$

Recordando que, dado que $x_{j}=x_{0}+j \mu_{0}$, la función de onda sólo puede tomar valores enteros, podemos reescribir de manera conveniente la ecuación (5.25):

$$
\psi(j+2)-\left(2-\frac{2 m E \mu_{0}^{2}}{\hbar^{2}}\right) \psi(j+1)+\psi(j)=0 .
$$

Siguiendo [186], la solución de una ecuación en diferencias de segundo orden es

$$
\psi(j)=a_{1} \Lambda_{1}^{j}+a_{2} \Lambda_{2}^{j} .
$$

donde $a_{i}$ son coeficientes constantes y $\Lambda_{i}$ son las raíces de la ecuación característica:

$$
\Lambda^{2}-\left(2-\frac{2 m E \mu_{0}^{2}}{\hbar^{2}}\right) \Lambda+1=0
$$

Las soluciones de (5.36) son de la forma

$$
\Lambda_{ \pm}=\left(1-\frac{m E \mu_{0}^{2}}{\hbar^{2}}\right) \pm \frac{1}{2} \sqrt{\frac{8 m E \mu_{0}^{2}}{\hbar^{2}}\left(\frac{m E \mu_{0}^{2}}{2 \hbar^{2}}-1\right)} .
$$

Para energías positivas, el argumento de la raíz cuadrada, nos da una relación entre la energía y $2 \hbar^{2} / m \mu_{0}^{2}$. Si $E \geq 2 \hbar^{2} / m \mu_{0}^{2}$, las raíces $\Lambda_{ \pm}$son números reales ${ }^{11}$, sin embargo, cuando se aplican las condiciones de frontera, ambas soluciones llevan a la solución trivial $\psi\left(x_{j}\right)=0$. Podemos pensar que la escala mínima $\mu_{0}$ impone un corte de energía, de tal forma que energías mayores que dicho corte no tienen sentido físico. El único caso físicamente significativo es cuando $E<2 \hbar^{2} / m \mu_{0}^{2}$ el cual nos da raíces complejas para $\Lambda_{ \pm}$, dado por [186]:

$$
\psi(j)=C_{1} \sin (j \theta)+C_{2} \cos (j \theta),
$$

esta es sólo una posible parametrización de (5.35) en coordenadas polares, donde $\cos \theta=\left(1-m E \mu_{0}^{2} / \hbar^{2}\right)$. Imponiendo las condiciones de frontera (5.33) en (5.38), encontramos que $\theta=n \pi \lambda$, en este caso $\lambda=\mu_{0} / L$ y $n \in \mathbb{Z}$. La eigenfunción de (5.34) puede escribirse como

$$
\psi_{n}\left(x_{j}\right)=C \sin \left(n \pi \lambda \frac{x_{j}}{\mu_{0}}\right)
$$

donde $C$ es un factor de normalización

$$
C=\left[\sum_{x_{j}} \sin ^{2}\left(n \pi \lambda \frac{x_{j}}{\mu_{0}}\right)\right]^{-\frac{1}{2}} .
$$

\footnotetext{
${ }^{11}$ El segundo caso lleva a raíces degeneradas para las cuales la solución (5.35) cambia a $\psi(j)=\Lambda^{j}\left(a_{1}+a_{2} j\right)$.
} 
Con (5.39) podemos decir que el correspondiente eigenestado (5.24) como

$$
\left|\psi_{n}\right\rangle=C \sum_{x_{j}} \sin \left(n \pi \lambda \frac{x_{j}}{\mu_{0}}\right)\left|x_{j}\right\rangle .
$$

Por otro lado encontramos que el espectro de energías puede escribirse como

$$
E_{n}=\frac{\hbar^{2}}{m \mu_{0}^{2}}(1-\cos n \pi \lambda) \text {. }
$$

Si desarrollamos $\cos n \pi \lambda$, para $\lambda \ll 1$, es decir, una longitud de la caja mucho mayor que la longitud mínima, hasta el tercer término, entonces recuperamos el espectro usual de la cuantización de Schrödinger con términos de corrección de orden $\mathcal{O}\left(\lambda^{2}\right)$

$$
E_{n}=\frac{\hbar^{2} n^{2} \pi^{2}}{2 m L^{2}}-\frac{\hbar^{2} n^{4} \pi^{4}}{24 m L^{2}} \lambda^{2}+\ldots
$$

Usando $\mu_{0}=10^{-19} \mathrm{~m}$ como en el caso anterior, y con el espectro aproximado (5.43), la corrección será significativa sólo para $n \approx 10^{17}$.

Podemos darnos cuenta de que, a diferencia del caso del oscilador, encontramos una expresión exacta para el espectro de energía (5.42), que está acotado por arriba y por abajo ${ }^{12}, 0<E_{n}<2 \hbar^{2} / m \mu_{0}^{2}$, como ya sabíamos. Del mismo modo que el caso anterior, podemos ver que el espectro aproximado (5.43) es válido únicamente cuando $n \lesssim \lambda^{-1}$, sin embargo, como veremos más adelante será necesario introducir un corte en $n$ incluso para el caso no aproximado.

El espectro de energía de una partícula cuántica en una caja se ha estudiado en el marco de una generalización del principio de incertidumbre (GUP) en el contexto de la fenomenología de la gravedad cuántica [185]. En ese caso las modificaciones inducidas por la escala mínima $\ell_{\min }$ en la función de onda y el espectro de energía de una partícula en una caja unidimensional, resultan en modificaciones a la ecuación de Schrödinger, transformándola en una ecuación diferencial de cuarto orden. El espectro de energía sólo puede encontrarse de forma aproximada, el término correctivo es proporcional al cuadrado de la longitud mínima $\ell_{\text {min }}^{2}$

$$
E_{n}=\frac{n^{2} \pi^{2} \hbar^{2}}{2 m L^{2}}+\frac{\ell_{\min }^{2}}{L^{2}} \frac{n^{4} \pi^{4} \hbar^{2}}{3 m L^{2}} .
$$

Este resultado puede compararse con (5.43). Podemos ver que la dependencia en la escala mínima es cuadrática en ambos casos, la única diferencia es que en (5.44), la corrección aumenta los niveles de energía mientras que (5.43) los disminuye. Por otro lado en [185] se usa la condición usual de frontera que sirve para contener la partícula libre en la caja, sin embargo, debido a que la medición de la posición tiene cierta incertidumbre que resulta proporcional a la longitud mínima, la posición de las paredes no se puede determinar de manera precisa. En el caso cuantizado por lazos esto se resolvió recordando que la dinámica está definida en un espacio superselecto para los que se definen, en cada uno, condiciones de frontera apropiadas dadas en (5.32) y (5.33).

\footnotetext{
${ }^{12}$ En realidad está cota fue obtenida ya para la partícula libre en [167].
} 


\subsection{Correcciones poliméricas a magnitudes termodinámicas de sistemas simples}

En mecánica estadística usual [81], la función de partición canónica $Z$ se define como la suma sobre todos los estados disponibles que tiene determinado sistema

$$
Z(\beta)=\sum_{n=0}^{\infty} \exp \left(-\beta E_{n}\right)
$$

donde $\beta=(k T)^{-1}, k$ es la constante de Boltzmann y $T$ es la temperatura. Esta función nos da información acerca de los posibles estados de energía a que el sistema puede acceder, es a través de esta función que nosotros podemos calcular todas las cantidades termodinámicas relevantes del sistema cuando se estudia macroscópicamente.

En esta sección utilizamos los espectros (5.29) y (5.43), encontrados en la sección anterior, para calcular la función de partición canónica (5.45) y luego obtener las cantidades termodinámicas de un ensamble de osciladores armónicos y de un gas ideal.

En este trabajo decidimos utilizar la estadística de Maxwell-Boltzmann para partículas sin espín, ya que es el caso más simple donde podemos estudiar los posibles cambios en las magnitudes termodinámicas que induce la estructura discreta espacial de la mecánica cuántica polimérica.

Se ha argumentado que las estadísticas modificadas pueden aparecer en la gravedad cuántica y las teorías discretas, sin embargo la relación entre la estructura discreta y la estadística sigue aún abierta [187].

\subsubsection{Ensamble de osciladores armónicos cuantizados por lazos}

Utilizando el espectro modificado (5.29) podemos calcular la función de partición canónica que en este caso correspondiente al oscilador armónico resulta ser

$$
Z(\beta)=\sum_{n=0}^{\infty} e^{-\beta \hbar \omega\left(\frac{1}{2}+n\right)} \exp \left[\frac{\lambda^{2}}{16} \beta \hbar \omega\left(\frac{1}{2}+n(n+1)\right)\right] .
$$

Dado que $\lambda \ll 1$, podemos desarrollar la segunda exponencial y considerar dicho desarrollo hasta orden $\lambda^{2}$, de modo tal que (5.46) puede expresarse como sigue

$$
Z(\beta)=e^{-\frac{\beta \hbar \omega}{2}}\left[\left(1+\lambda^{2} \frac{\beta \hbar \omega}{32}\right) \sum_{n=0}^{\infty} e^{-\beta \hbar \omega n}+\frac{\lambda^{2}}{16} \beta \hbar \omega \sum_{n=0}^{\infty}\left(n^{2}+n\right) e^{-\beta \hbar \omega n}+\mathcal{O}\left(\lambda^{4}\right)\right] .
$$

Sabemos que la corrección polimérica no es válida para todos los valores de $n$, sin embargo, si es válida para $n$ suficientemente grande, de modo que realizar la suma hasta $\infty$ pretende ser solamente una aproximación. Con estas consideraciones la función de partición se convierte en

$$
Z(\beta) \simeq \frac{e^{-\frac{\beta \hbar \omega}{2}}}{1-e^{-\beta \hbar \omega}}\left[1+\frac{\lambda^{2}}{32} \beta \hbar \omega\left(\frac{1+e^{-\beta \hbar \omega}}{1-e^{-\beta \hbar \omega}}\right)^{2}+\mathcal{O}\left(\lambda^{4}\right)\right]
$$


Podemos reconocer el primer término en (5.48) como la función de partición habitual del ensamble de osciladores armónicos, mientras que el segundo término es la corrección polimérica. Como sabemos, el primer término debe ser mucho mayor que la corrección, entonces debe haber una relación entre $\lambda$ y $\beta$ para que la aproximación sea válida. Esto nos da la siguiente desigualdad

$$
\lambda<\lambda_{c}, \quad \text { donde } \quad \lambda_{c}=\sqrt{\frac{32}{\beta \hbar \omega}}\left(\frac{1-e^{-\beta \hbar \omega}}{1+e^{-\beta \hbar \omega}}\right),
$$

teniendo en cuenta que, además de la condición anterior hay que asegurarse de que $\lambda \ll 1$, entonces la aproximación es válida para los casos extremos, es decir, $\beta \hbar \omega \gg 1$ y $\beta \hbar \omega \ll 1$.

Ahora, podemos calcular las cantidades termodinámicas a través de la relación entre la función de partición y la energía libre de Helmholtz [81]

$$
F=-\frac{\mathcal{N}}{\beta} \ln Z
$$

donde $\mathcal{N}$ es el número de partículas. Utilizando (5.48) podemos escribir la energía libre de Helmholtz con las modificaciones poliméricas

$$
F=\frac{\mathcal{N}}{\beta}\left[\frac{\beta \hbar \omega}{2}+\ln \left(1-e^{-\beta \hbar \omega}\right)-\ln \left(1+\frac{\lambda^{2}}{\lambda_{c}^{2}}\right)\right] \cong \frac{\mathcal{N}}{\beta}\left[\frac{\beta \hbar \omega}{2}+\ln \left(1-e^{-\beta \hbar \omega}\right)-\frac{\lambda^{2}}{\lambda_{c}^{2}}\right]
$$

Con (5.51) podemos calcular varias cantidades como el potencial químico, la presión y la entropía, a través de las bien conocidas relaciones

$$
\begin{aligned}
\mu & =\frac{\partial F}{\partial \mathcal{N}}, \\
\mathcal{P} & =-\frac{\partial F}{\partial L}, \\
\mathcal{S} & =k \beta^{2} \frac{\partial F}{\partial \beta} .
\end{aligned}
$$

Sustituyendo (5.51) en (5.52)-(5.54) obtenemos: $\mu=F / \mathcal{N}$ y $\mathcal{P}=0$. Es interesante que la ecuación de estado a este orden en la aproximación no se ve modificada respecto a la de un sólido de Einstein. Es posible que existan modificaciones al considerar más términos correctivos en el espectro, o bien una aproximación distinta en (5.46) o más términos en (5.47). La entropía es

$$
\mathcal{S}=\mathcal{N} k\left[\frac{\beta \hbar \omega}{e^{\beta \hbar \omega}-1}-\ln \left(1-e^{-\beta \hbar \omega}\right)+\frac{(\lambda \beta \hbar \omega)^{2}}{8} e^{\beta \hbar \omega} \frac{e^{\beta \hbar \omega}+1}{\left(e^{\beta \hbar \omega}-1\right)^{3}}\right] .
$$

También podemos calcular la energía interna y la capacidad calorífica a volumen constante con las siguientes relaciones

$$
\begin{aligned}
U & =-\mathcal{N} \frac{\partial \ln Z}{\partial \beta}=-\frac{\mathcal{N}}{Z} \frac{\partial Z}{\partial \beta}, \\
C_{V} & =-k \beta^{2} \frac{\partial U}{\partial \beta} .
\end{aligned}
$$


Para la energía tenemos

$$
U=\mathcal{N} \hbar \omega\left[\frac{1}{2}+\frac{1}{e^{\beta \hbar \omega}-1}-\frac{\lambda^{2}}{32} \frac{\left(1+e^{\beta \hbar \omega}\right)\left(e^{2 \beta \hbar \omega}-4 e^{\beta \hbar \omega} \beta \hbar \omega-1\right)}{\left(e^{\beta \hbar \omega}-1\right)^{3}}\right]
$$

mientras que para la capacidad calorífica

$$
C_{V}=\mathcal{N} k(\beta \hbar \omega)^{2} \frac{e^{\beta \hbar \omega}}{\left(e^{\beta \hbar \omega}-1\right)^{2}}\left[1+\frac{\lambda^{2}}{8}\left(\frac{2+\beta \hbar \omega\left(1+4 e^{\beta \hbar \omega}\right)+e^{2 \beta \hbar \omega}(\beta \hbar \omega-2)}{\left(e^{\beta \hbar \omega}-1\right)^{2}}\right)\right] .
$$

De la expresión (5.59) nos damos cuenta de que la corrección de la cuantización por lazos aumenta la capacidad calorífica. Para $\beta \hbar \omega \gg 1$, la corrección es proporcional a $\left(\lambda / \lambda_{c}\right)^{2}$, que es menor que 1 . En el caso de que $\beta \hbar \omega \ll 1$, la corrección es proporcional a $\lambda^{2} /(\beta \hbar \omega)^{3}$, que no puede despreciarse para $\beta \hbar \omega \sim \lambda^{2 / 3}$. Utilizando los valores considerados en la sección anterior para el monóxido de carbono, la corrección será significativa para $\beta \sim 10^{14}$. Si nos fijamos en la corrección de la energía interna vemos que desaparece cuando $e^{2 \beta \hbar \omega}=4 e^{\beta \hbar \omega} \beta \hbar \omega+1$. Entonces hay una temperatura $\beta \sim 10^{19}$ en el que este ensamble no percibe la estructura discreta del espacio, una ligera disminución o aumento de la temperatura alrededor de este valor podría reflejarse en una disminución o incremento correspondiente de la energía. Por otra parte, notamos que la corrección de la cuantización por lazos disminuye el valor usual de la energía interna, cuando $\beta \hbar \omega \gg 1$ la corrección es proporcional a $-\left(\lambda / \lambda_{c}\right)^{2}$. Para $\beta \hbar \omega \ll 1$ la corrección resulta ser positiva y proporcional a $(\lambda / \beta \hbar \omega)^{2}$. De nuevo para $\beta \sim 10^{12}$ la corrección puede ser importante.

Físicamente un ensamble de osciladores armónicos se ha utilizado para modelar vibraciones en sólidos (fonones) y en el modelos más sencillo de la radiación de cuerpo negro (fotones). Si utilizamos estos resultados interpretándolos como alguno de estos sistemas podemos obtener algunos resultados interesantes.

\section{Sólido de Einstein modificado}

En un sólido los modos normales de vibración se modelan como una colección de osciladores armónicos en la llamada aproximación armónica la cual consiste en aproximar el Hamiltoniano clásico de interacción de los átomos de un sólido por un desarrollo de Taylor hasta segundo orden, de ahí el término armónico [81]. El modelo más sencillo es el llamado modelo de Einstein el cual supone que todos los modos de vibración tienen la misma frecuencia $\omega$, que los osciladores son independientes y sin interacción. Las variables termodinámicas (5.51), (5.55), (5.58) y (5.59) contienen entonces las modificaciones a la termodinámica de un sólido de Einstein introduciendo la temperatura vibracional $\Theta_{v}=\hbar \omega / k$.

Una posible consecuencia puede observarse en la energía interna y en la capacidad calorífica. En la figura 5.2 se muestran gráficas cualitativas de estas cantidades. Puede apreciarse como mientras la energía interna y la capacidad calorífica no modificadas tienen un comportamiento asintótico conocido, las correspondientes $U$ y $C_{V}$ con correcciones debidas a la escala mínima difieren de las anteriores (aunque muy poco) en la región de temperaturas altas. 


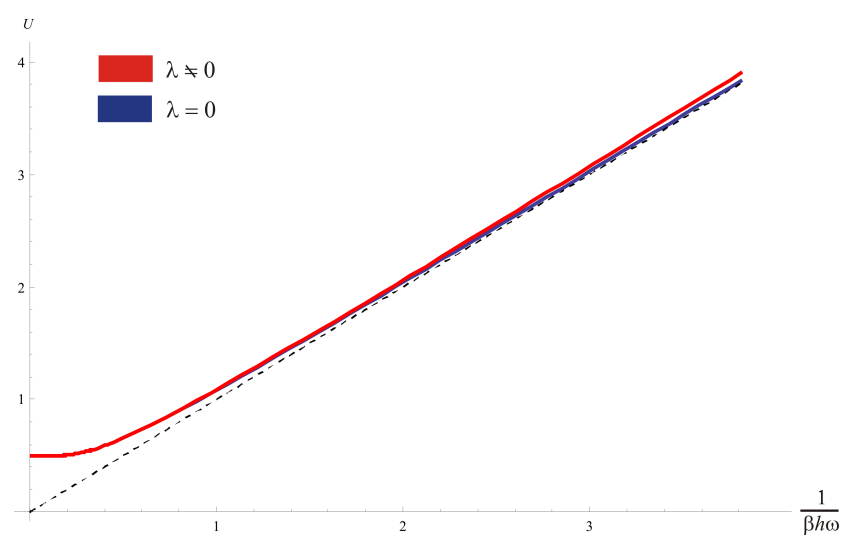

(a)

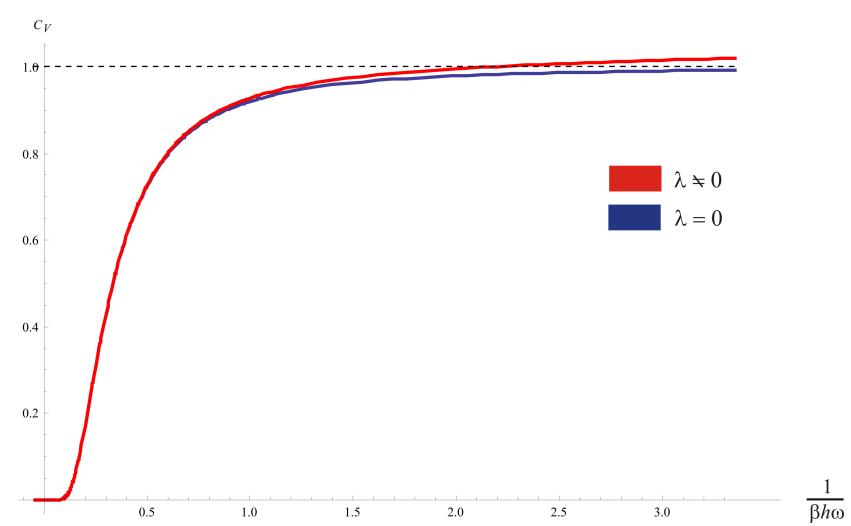

(b)

Figura 5.2: Gráficas cualitativas de la energía interna $U$ (a) y la capacidad calorífica $C_{V}$ (b) contra $1 / \beta \hbar \omega$ para el sólido de Einstein usual (azul) y el modificado poliméricamente (rojo).

\section{Modelo simple para la radiación de cuerpo negro}

Históricamente la radiación en equilibrio se ha estudiado con dos modelos distintos conceptualmente [29, 81, 127]: En primer lugar Planck en 1900 consideró este sistema como un ensamble de osciladores armónicos con la misma frecuencia y energías individuales que sólo podían ocurrir como múltiplos de cierta constante, es decir, energías cuantizadas [188]. Por otra parte Bose y Einstein alrededor de 1924, modelaron este sistema como un gas de partículas idénticas llamadas fotones que tienen cierta energía particular $\hbar \omega$, en el lenguaje actual decimos que los fotones son bosones ultrarelativistas.

Adoptemos para un análisis simple el modelo de Planck. Si interpretamos a la energía interna como $\mathcal{N}$ veces la energía promedio de cada oscilador, es decir, $U=\mathcal{N}\left\langle E_{n}\right\rangle$, de donde se sigue que $\left\langle E_{n}\right\rangle=\frac{\hbar \omega}{2}+\langle n\rangle$, donde $\langle n\rangle$ se conoce como el número de ocupación promedio y en este caso es

$$
\langle n\rangle=\frac{1}{e^{\beta \hbar \omega}-1}-\frac{\lambda^{2}}{32} \frac{\left(1+e^{\beta \hbar \omega}\right)\left(e^{2 \beta \hbar \omega}-4 \beta \hbar \omega e^{\beta \hbar \omega}-1\right)}{\left(e^{\beta \hbar \omega}-1\right)^{3}},
$$

donde el último término contiene la corrección polimérica. Con (5.60) podemos calcular la densidad espectral que se define como

$$
u_{\omega} \equiv \frac{d}{d \omega}\left(\frac{U}{V}\right)=\langle n\rangle \hbar \omega \frac{g(\omega)}{V}
$$

donde la densidad de estados usual $g(\omega)=\frac{V \omega^{2}}{\pi^{2} c^{3}}$, que se utilizará para obtener resultados aproximados. La expresión (5.61) es la distribución de cuerpo negro que dado (5.60) tendrá correcciones poliméricas de orden $\mathcal{O}\left(\lambda^{2}\right)$. Este resultado puede compararse con aquel en [189] que resulta de considerar una relación de dispersión modificada por efectos de la gravedad cuánticas de lazos [178, 177], o también con [190] donde se utilizan términos más generales que engloban diversas propuestas de gravedad cuántica, puede verificarse que el orden de las correcciones en la escala es similar. 
El máximo de (5.61), que sigue la bien conocida ley de desplazamiento de Wien [81, 29, 127, 188], donde el máximo está dado por $\beta \hbar \omega=2.82144$, resulta interesante que incluyendo correcciones poliméricas este máximo se ve modificado, y aunque no se tiene una expresión analítica como en [189, 190] puede calcularse numéricamente para diversos valores de $\lambda$, así por ejemplo para $\lambda=0.01$ el máximo estará en 2.8213. Es claro que para $\lambda$ del orden de la escala de Planck la corrección será mucho más pequeña.

Utilizando (5.60) y (5.61) puede obtenerse directamente la densidad de energía que se sabe en el caso usual sin modificaciones poliméricas sigue la ley de Stefan-Boltzmann. En este caso se obtiene

$$
\frac{U}{V}=\frac{\pi^{2} k^{4}}{15 c^{3} \hbar^{3}}\left(1+\lambda^{2} \frac{135}{4 \pi^{4}} \zeta(3)\right) T^{4} \simeq \frac{\pi^{2} k^{4}}{15 c^{3} \hbar^{3}}\left(1+\lambda^{2} 0.416485\right) T^{4}
$$

notemos que a diferencia de otros enfoque $[189,190]$ en este caso las modificaciones dependen únicamente de la escala y no de la temperatura. La dependencia en la temperatura resulta ser la misma $T^{4}$.

Si los fotones están en una cavidad y hay una pequeña abertura en las paredes de esta, los fotones efunden a través de la abertura. La tasa neta de flujo de la radiación, por unidad de área de la apertura, se dada por el producto de la densidad de energía por $c / 4$, dado que $c$ es la velocidad de los fotones, $\Phi=\sigma_{S B} T^{4}$. De esta forma, dado (5.62) la constante de Stefan-Boltzmann se ve modificada.

$$
\sigma_{S B}^{(\lambda)}=\frac{\pi^{2} k^{4}}{60 c^{2} \hbar^{3}}\left(1+\lambda^{2} 0.416485\right)
$$

Nótese que $\lambda$ es necesariamente pequeña ya que si $\sigma_{S B}^{\lambda}$ fuera muy grande se tendría otra catástrofe ultravioleta. Notemos que dado (5.62) las correspondientes modificaciones a las cantidades termodinámicas no dependerán de la temperatura como en [189, 190] sino únicamente en la escala.

Finalmente si se grafica la distribución de cuerpo negro o densidad espectral para alguna temperatura fija usando (5.60) y (5.61) se puede observar en Fig. 5.3 que para altas frecuencias la distribución se modifica. Sabemos que la radiación cósmica de fondo con $T \cong 3 K$ (en el marco comóvil con la radiación) es el cuerpo negro que se ha medido en la naturaleza con mayor precisión y con incertidumbres muy pequeñas [98]. De nuevo este hecho constriñe aún más el posible valor de $\lambda$ y por tanto el de la escala mínima $\mu_{0}$. Notemos dos puntos importantes, en este modelo hemos ignorado completamente la energía del estado base ya que al integrarse para obtener la densidad de energía daría un término divergente. Además, la presión predicha por este modelo no corresponde a la presión de radiación $\mathcal{P}=U / 3 \mathrm{~V}$ conocida. Estos dos puntos los resuelve la distribución de Bose-Einstein en el caso estándar. Esto apunta a que sería interesante extender este análisis al caso de funciones de partición para otros ensambles como el ensamble gran canónico como se suguiere en [189]. 


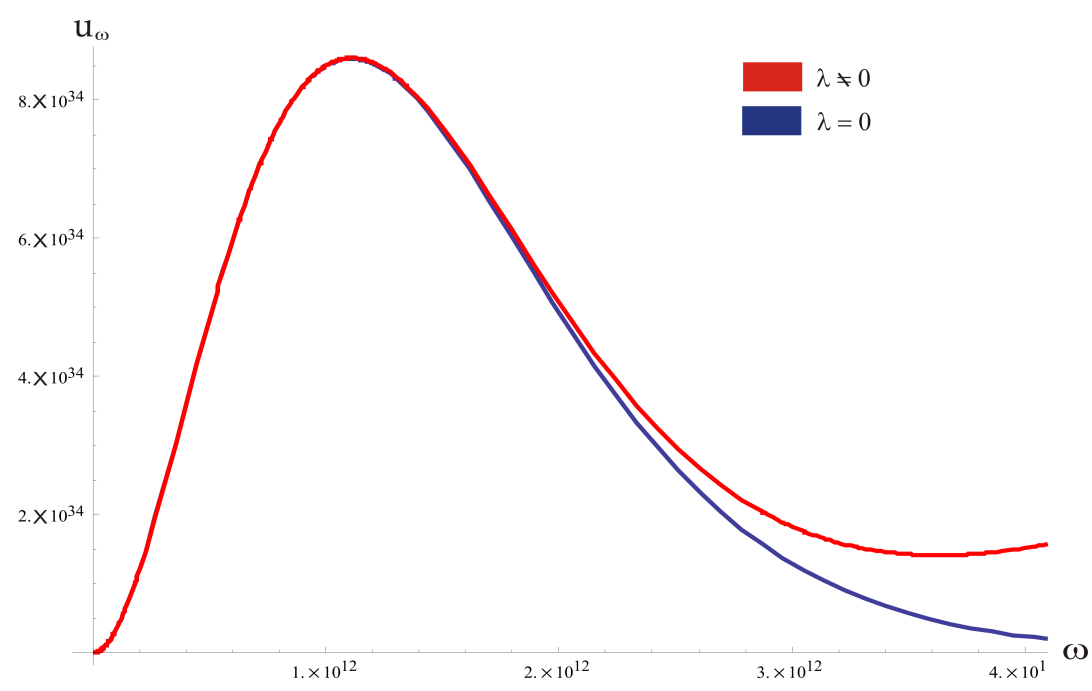

Figura 5.3: Comparación de la distribución de cuerpo negro o densidad espectral usual (azul) contra la distribución con modificaciones poliméricas de orden $\mathcal{O}\left(\lambda^{2}\right)$ (rojo), ambas para una temperatura de $T=3 K$.

\subsubsection{Función de partición para el Gas Ideal polimérico}

En la sección anterior encontramos que el espectro de energía de una partícula cuántica polimérica, en una caja podía escribirse como (5.42)

$$
E_{n}=\frac{\hbar^{2}}{m \mu_{0}^{2}}(1-\cos n \pi \lambda)
$$

también mostramos que si desarrollamos $\cos n \pi \lambda$, en el caso en que $\lambda \ll 1$, es decir, si las dimensiones de la caja son grandes en comparación con la escala mínima, entonces recuperábamos el espectro cuántico usual con modificaciones del orden $\mathcal{O}\left(\lambda^{2}\right)$ :

$$
E_{n} \cong \frac{\hbar^{2} n^{2} \pi^{2}}{2 m L^{2}}-\frac{\hbar^{2} n^{4} \pi^{4}}{24 m L^{2}} \lambda^{2}+\ldots
$$

De la misma manera como en el caso anterior correspondiente al ensamble de osciladores podemos calcular la función de partición con el espectro de energía de la partícula en la caja, sustituyendo alguna (5.64) o (5.65) en la suma (5.45).

Utilizando (5.65) entonces $Z$ resulta

$$
Z(\beta)=\sum_{n=0}^{\infty} \exp \left[-\beta\left(\frac{\hbar^{2} n^{2} \pi^{2}}{2 m L^{2}}-\frac{\hbar^{2} n^{4} \pi^{4}}{24 m L^{2}} \lambda^{2}+\ldots\right)\right],
$$

donde estamos considerando de nuevo todos los valores de $n$ como una aproximación. Para continuar, utilizamos el hecho de que $\lambda \ll 1$ y así considerar sólo los primeros términos de la serie correspondiente 
al segundo factor exponencial con argumento proporcional a $\lambda^{2}$, de modo tal que la función de partición puede expresarse como

$$
Z(\beta)=\sum_{n=0}^{\infty} e^{-\frac{\beta \hbar^{2} \pi^{2}}{2 m L^{2}} n^{2}}+\lambda^{2} \frac{\beta \hbar^{2} \pi^{4}}{24 m L^{2}} \sum_{n=0}^{\infty} n^{4} e^{-\frac{\beta \hbar^{2} \pi^{2}}{2 m L^{2}} n^{2}}+\mathcal{O}\left(\lambda^{4}\right) .
$$

Por otra parte dado que $\beta \hbar^{2} \pi^{2} / 2 m L^{2} \approx 10^{-16}$, la exponencial de (5.67) decae muy lentamente y por lo tanto no es posible estimar el valor de la suma considerando sólo algunos términos. Sin embargo, podemos utilizar la fórmula de re-suma de Poisson para aproximar ambas sumas en (5.67):

$$
\sum_{n=-\infty}^{\infty} f(n)=\sum_{n=-\infty}^{\infty} \int_{-\infty}^{\infty} f(y) e^{-2 \pi i y n} d y
$$

De este modo la función de partición puede ser escrita como ${ }^{13}$

$$
Z(\beta) \cong \sqrt{\frac{m L^{2}}{2 \pi \beta \hbar^{2}}}+\lambda^{2} \frac{\pi}{4}\left(\frac{m L^{2}}{2 \pi \beta \hbar^{2}}\right)^{3 / 2}+\mathcal{O}\left(e^{-\frac{2 m L^{2}}{\beta \hbar^{2}}}, \lambda^{4}\right) .
$$

Al igual que en el caso del ensamble de osciladores el primer término es la función de partición estándar para el gas ideal unidimensional. Este término principal nos lleva a una relación entre $\beta$ y $\lambda$ que nos da los límites de validez de nuestra aproximación

$$
\lambda \ll \sqrt{\frac{8 \beta \hbar^{2}}{m L^{2}}} .
$$

Calculemos ahora la energía libre de Helmholtz ${ }^{14}$

$$
F=\frac{-\mathcal{N}}{\beta}\left[1-\ln \mathcal{N}+\ln L-\frac{1}{2} \ln \beta+\ln \sqrt{\frac{m}{2 \pi \hbar^{2}}}+\ln \left(1+\frac{\lambda^{2}}{8} \frac{m L^{2}}{\beta \hbar^{2}}\right)\right] .
$$

De estas expresiones podemos notar que $F$ diverge para $\beta=0$ o $T \rightarrow \infty$, pero con signo contrario al del caso habitual. Al acercarnos a $\beta=0$ encontramos un máximo después del que la energía libre diverge a $-\infty$ en $\beta=0$. Esto significa que el efecto en la energía libre de la escala de longitud mínima, es proporcionar un punto de inflexión para $F$ a temperaturas muy altas. Por otra parte, cuando aumentamos el valor de $\mu_{0}$, la energía libre se convierte en negativa a altas temperaturas. Esto impone una restricción sobre los valores que $\mu_{0}$ puede tomar.

Podemos calcular la presión del gas a través de la relación de $\mathcal{P}=-\frac{\partial F}{\partial L}$, recordando que estamos analizando un sistema en una sola dimensión. Al darnos cuenta de que el término que surge de la corrección de la cuantización por lazos no depende de $\lambda$, pero en $\mu_{0}$, podemos obtener la misma ecuación de estado del gas ideal en una dimensión $\mathcal{P}=\mathcal{N} / \beta L$, como en el caso continuo. Lo mismo sucede en el caso anterior,

\footnotetext{
${ }^{13}$ Consideramos sólo el primer término de la suma que corresponde al caso de la función de partición del gas ideal. Como queremos ver las correcciones a las cantidades termodinámicas del gas ideal, entonces (5.69) es una buena aproximación.

${ }^{14}$ En este caso la definición de $F$ incluye un término $\mathcal{N}$ !, como sucede para partículas indistinguibles [81].
} 
esto nos dice que las correcciones poliméricas no pueden pensarse como una interacción efectiva entre las partículas, como sucede con las propuestas de generalizar el principio de incertidumbre (GUP) o modificar las relaciones de dispersión (MDR) [191].

Recordando (5.53) y (5.54) el potencial químico y la entropía del gas se pueden calcular dando las siguientes expresiones

$$
\begin{gathered}
\mu=-\frac{1}{\beta}\left[-\ln \mathcal{N}+\ln L-\frac{1}{2} \ln \beta+\ln \sqrt{\frac{m}{2 \pi \hbar^{2}}}+\ln \left(1+\frac{\lambda^{2}}{8} \frac{m L^{2}}{\beta \hbar^{2}}\right)\right], \\
\mathcal{S}=\mathcal{N} k\left[\frac{3}{2}+\ln \frac{L}{\mathcal{N}}-\ln \beta^{1 / 2}+\ln \sqrt{\frac{m}{2 \pi \hbar^{2}}}+\frac{\lambda^{2} m L^{2}}{8 \hbar^{2} \beta}+\ln \left(1+\frac{\lambda^{2} m L^{2}}{8 \hbar^{2} \beta}\right)\right] .
\end{gathered}
$$

La última expresión sería el equivalente en una dimensión a la fórmula de Sackur-Tetrode para la entropía [29] con modificaciones debidas a la discretez subyacente. La energía y la capacidad calorífica se obtienen de (5.56) y (5.57), respectivamente:

$$
\begin{aligned}
U & =\frac{\mathcal{N}}{2 \beta}\left(1+\frac{\lambda^{2}}{4} \frac{m L^{2}}{\hbar^{2} \beta}\right), \\
C_{V} & =\frac{k \mathcal{N}}{2}\left(1+\frac{\lambda^{2}}{2} \frac{m L^{2}}{\hbar^{2} \beta}\right) .
\end{aligned}
$$

De la expresión (5.75) nos damos cuenta de que la capacidad calorífica diverge en $\beta=0$, esto difiere del caso continuo usual donde $C_{V}$ es constante. Como $C_{V}$ es la segunda derivada de $F$, entonces, la divergencia de $C_{V}$ puede interpretarse como una transición de segundo orden a temperatura infinita [192]. Ver Fig. 5.4. Por supuesto que no podemos acceder a esta transición, lo que podemos decir es que para altas temperaturas, se produce un aumento en $C_{V}$ con respecto al caso usual. Como la capacidad calorífica está relacionada con las fluctuaciones de la energía, podemos decir que para las altas temperaturas hay fuertes fluctuaciones en la energía debida a la estructura discreta del espacio.

Tomemos en cuenta que si usamos el resultado (5.44) los cálculos de las cantidades termodinámicas siguen siendo los mismos y sólo se diferencian por factores numéricos, es decir, en la función de partición (5.67) un factor de $1 / 24$ se sustituye por $-1 / 3$. La principal diferencia es que con (5.44), asociado a la modificación de la relación de incertidumbre, las cantidades termodinámicas disminuyen en lugar de aumentar en contraste con nuestros resultados.

No obstante, al haber escogido el espectro aproximado (5.65) los resultados anteriores sólo son válidos en la misma aproximación en la que (5.65) es válido, es decir (5.70). Es importante destacar que esta primera aproximación muestra de manera clara como los resultados conocidos de termodinámica en espacios continuos, están contenidos en la formulación de la cuantización por lazos. Si buscáramos una aproximación más precisa una posibilidad es considerar más términos en la fórmula de re-suma de Poisson, digamos a orden $\lambda^{8}$, en tal caso tendríamos

$$
Z(\beta) \cong \frac{L}{x}\left[1+\frac{\pi}{4} \lambda^{2} \frac{L^{2}}{x^{2}}+\frac{9 \pi^{2}}{32} \lambda^{4} \frac{L^{4}}{x^{4}}+\frac{75 \pi^{3}}{128} \lambda^{6} \frac{L^{6}}{x^{6}}+\frac{3675 \pi^{4}}{2048} \lambda^{8} \frac{L^{8}}{x^{8}}+\mathcal{O}\left(e^{-\frac{2 m L^{2}}{\beta \hbar^{2}}}, \lambda^{10}\right)\right],
$$




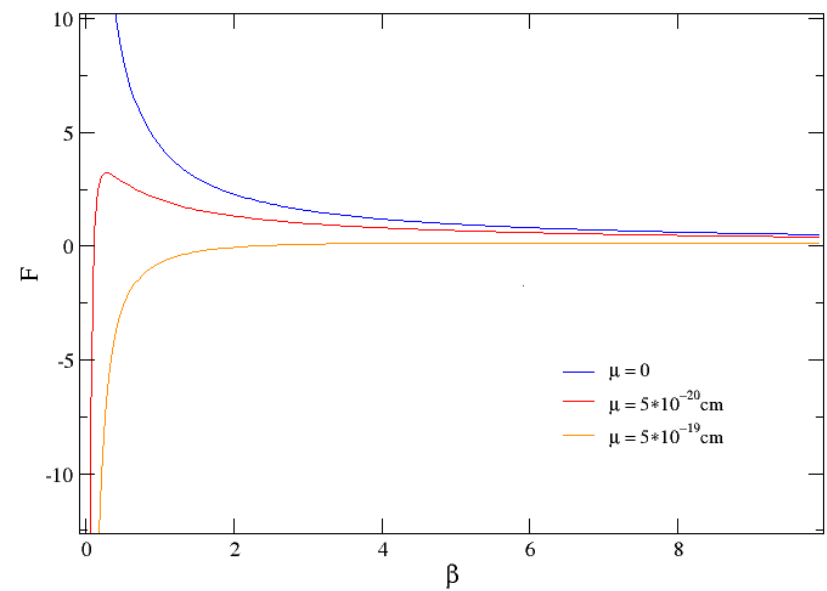

(a)

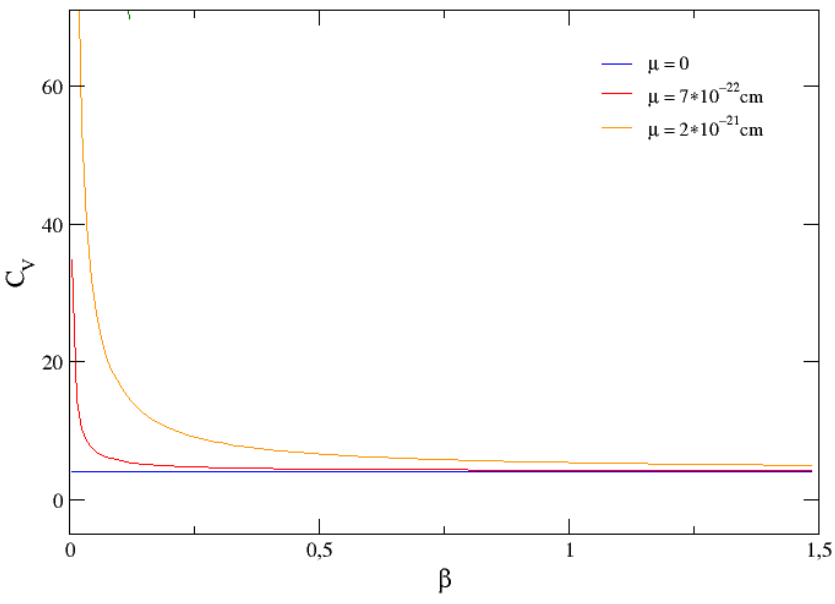

(b)

Figura 5.4: Gráficas de (a) la energía libre $F$ y (b) capacidad calorífica del gas ideal con correcciones poliméricas a segundo orden en $\lambda=\mu_{0} / L$, para distintos valores de $\mu_{0}$. En ambas gráficas puede apreciarse una transición de fase a temperaturas altas $\beta=0$.

donde abreviamos $x=\sqrt{\frac{2 \pi \beta \hbar^{2}}{m}}$ que se identifica como la longitud térmica [81]. De esta manera podemos ver que la serie es en realidad independiente de $L$ y se puede escribir como

$$
Z(\beta) \cong \frac{L}{x}\left[1+\frac{\pi}{4} \frac{\mu_{0}^{2}}{x^{2}}+\frac{9 \pi^{2}}{32} \frac{\mu_{0}^{4}}{x^{4}}+\frac{75 \pi^{3}}{128} \frac{\mu_{0}^{6}}{x^{6}}+\frac{3675 \pi^{4}}{2048} \frac{\mu_{0}^{8}}{x^{8}}+\mathcal{O}\left(e^{-4 \pi \frac{L^{2}}{x^{2}}}, \mu_{0}^{10}\right)\right]
$$

nótese que ahora hay una nueva cantidad sin dimensiones, el cociente $\mu_{0} / x$.

Podemos calcular la energía libre de Helmholtz $F=-\beta^{-1} \ln \left(\frac{Z^{\mathcal{N}}}{\mathcal{N} !}\right)$ :

$$
F=\frac{-\mathcal{N}}{\beta}[1-\ln \mathcal{N}+\ln Z],
$$

donde, en este caso, considerando la aproximación (5.77), la cantidad $\ln Z$ es

$$
\ln Z \cong \ln \left(\frac{L}{x}\right)+\left[\frac{\pi}{4} \frac{\mu_{0}^{2}}{x^{2}}+\frac{\pi^{2}}{4} \frac{\mu_{0}^{4}}{x^{4}}+\frac{25 \pi^{3}}{48} \frac{\mu_{0}^{6}}{x^{6}}+\frac{13 \pi^{4}}{8} \frac{\mu_{0}^{8}}{x^{8}}+\mathcal{O}\left(-4 \pi \frac{L^{2}}{x^{2}}, \mu_{0}^{10}\right)\right]
$$

Claramente las expresiones (5.78) y (5.79) corrigen (5.71) hasta términos de orden $\mu_{0}^{8}$. Sin embargo, no hay diferencia en la forma de los términos de corrección, sólo tenemos más términos. La diferencia aparecerá sólo en las cantidades que estén relacionadas a través de derivadas respecto a la temperatura, pero de nuevo la forma es muy similar y al ser términos de un orden menor, las correcciones serán cada vez más pequeñas y por tanto despreciables.

Ahora bien, para tener una aproximación aún más precisa podemos recordar que, a diferencia del caso anterior del ensamble de osciladores, en este caso tenemos acceso al espectro completo sin aproximar (5.64), 
por lo tanto es inmediato preguntarnos sobre la función de partición y las cantidades termodinámicas que obtenemos con este espectro. En tal caso habría que calcular la función de partición

$$
Z(\beta)=\sum_{n=0}^{\infty} \exp \left[-\frac{\beta \hbar^{2}}{m \mu_{0}^{2}}(1-\cos n \pi \lambda)\right]
$$

las cantidades termodinámicas están relacionadas con el logaritmo natural de (5.80) que es

$$
\ln Z=\ln \left\{\sum_{n=0}^{\infty} \exp \left[-\frac{\beta \hbar^{2}}{m \mu_{0}^{2}}(1-\cos n \pi \lambda)\right]\right\} .
$$

Un posible camino es calcular las derivadas asociadas a las diferentes cantidades termodinámicas y evaluar las sumas en (5.80) y (5.81) para después obtener una expresión para cada cantidad termodinámica. La idea es mantener en la medida de lo posible una expresión cerrada como (5.80), aunque en forma de sumas, y luego aproximar.

Entonces ahora podemos calcular la ecuación de estado, es decir, la presión del gas, derivando la energía libre de Helmholtz de (5.78) y (5.81) con respecto a $L$, tal que la expresión cerrada es

$$
\mathcal{P}=\frac{N \hbar^{2} \pi}{L^{2} \mu_{0} m} \frac{\sum_{n} n \operatorname{sen}(n \pi \lambda) e^{-\frac{\beta \hbar^{2}}{m \mu_{0}^{2}}(1-\cos n \pi \lambda)}}{\sum_{n} e^{-\frac{\beta \hbar^{2}}{m \mu_{0}^{2}}(1-\cos n \pi \lambda)}} .
$$

En esta expresión es evidente que hay cambios debido a la estructura discreta del espacio. Si ahora aproximamos la suma en esta expresión lo que obtenemos es el siguiente

$$
\mathcal{P} \cong \frac{N}{\beta L^{2}}\left[1+\frac{11 \pi^{2}}{12} \frac{\mu_{0}^{4}}{x^{4}}-\frac{41 \pi^{3}}{384} \frac{\mu_{0}^{6}}{x^{6}}+\ldots\right]
$$

En contraste con la aproximación anterior, en este caso podemos ver la primera corrección a la ecuación de estado conocida, que es del orden $\mu_{0}^{4}$, y por lo tanto no podía ser detectada antes. Por otra parte, utilizando la expresión aproximada para la función de partición hasta orden $\mu_{0}^{8}$ (5.77), no habíamos obtenido modificaciones en la ecuación de estado, porque las correcciones en $Z$ no dependían de $L$. Debido a nuestra forma de aproximación de $Z$ no podíamos ver las correcciones en (5.83). Esto se vuelve aún más evidente si tenemos en cuenta que la primera corrección en la aproximación de Poisson a la suma sí depende de $L$.

Aunque a la corrección en $\mathcal{P}$ (5.83) es muy pequeña, puede ser interpretada como proveniente de una interacción atractiva entre las partículas de gas, de acuerdo con el desarrollo del Virial [81]. Es decir, la existencia de una escala de longitud mínima se refleja en la termodinámica del gas mediante la inducción de una pseudointeracción atractiva. Este tipo de análisis ha sido estudiado en un trabajo sobre la fenomenología de la gravedad cuántica que propone una posible violación a la simetría de Lorentz [191]. 
Podemos calcular las cantidades termodinámicas restantes de la misma manera como en la sección anterior. La expresión para la entropía $S=k \beta^{2} \frac{\partial F}{\partial \beta}$, en términos de sumas es:

$$
\mathcal{S}=k \mathcal{N}\left[1-\ln \mathcal{N}+\ln Z+\frac{\beta \hbar^{2}}{m \mu_{0}^{2}} \frac{\sum_{n}(1-\cos n \pi \lambda) e^{-\frac{\beta \hbar^{2}}{m \mu_{0}^{2}}(1-\cos n \pi \lambda)}}{\sum_{n} e^{-\frac{\beta \hbar^{2}}{m \mu_{0}^{2}}(1-\cos n \pi \lambda)}}\right]
$$

aproximando las sumatorias

$$
\mathcal{S} \cong \mathcal{N} k\left[\frac{3}{2}-\ln \mathcal{N}+\ln \frac{L}{x}+\frac{\pi}{2} \frac{\mu_{0}^{2}}{x^{2}}\left\{1+\frac{\pi 27}{2} \frac{\mu_{0}^{2}}{x^{2}}+\frac{\pi 127}{4} \frac{\mu_{0}^{4}}{x^{4}}+\ldots\right\}\right]
$$

que a su vez es una función homogénea, pero ahora al orden $\mu_{0}^{4}$. Sin embargo la ecuación (5.84) no es una función homogénea en $L$ y por lo tanto la entropía no puede ser una cantidad extensiva sin hacer la aproximación.

La energía interna $U=-\mathcal{N} \frac{\partial \ln Z}{\partial \beta}$ en este caso

$$
U=\frac{\mathcal{N} \hbar^{2}}{m \mu_{0}^{2}} \frac{\sum_{n}(1-\cos n \pi \lambda) e^{-\frac{\beta \hbar^{2}}{m \mu_{0}^{2}}(1-\cos n \pi \lambda)}}{\sum_{n} e^{-\frac{\beta \hbar^{2}}{m \mu_{0}^{2}}(1-\cos n \pi \lambda)}}
$$

y con la aproximación

$$
U \cong \frac{\mathcal{N}}{2 \beta}\left(1+\frac{\pi}{2} \frac{\mu_{0}^{2}}{x^{2}}+\frac{23 \pi^{2}}{8} \frac{\mu_{0}^{4}}{x^{4}}+\frac{281 \pi^{3}}{96} \frac{\mu_{0}^{6}}{x^{6}}+\ldots\right)
$$

Para la capacidad calorífica $C_{V}=-k \beta^{2} \frac{\partial U}{\partial \beta}$ tenemos

$$
C_{V}=\mathcal{N} k \beta^{2}\left(\frac{\hbar^{2}}{m \mu_{0}^{2}}\right)^{2}\left[\frac{\sum_{n}(1-\cos n \pi \lambda)^{2} e^{-\frac{\beta \hbar^{2}}{m \mu_{0}^{2}}(1-\cos n \pi \lambda)}}{\sum_{n} e^{-\frac{\beta \hbar^{2}}{m \mu_{0}^{2}}(1-\cos n \pi \lambda)}}-\frac{\left(\sum_{n}(1-\cos n \pi \lambda)^{2} e^{-\frac{\beta \hbar^{2}}{m \mu_{0}^{2}}(1-\cos n \pi \lambda)}\right)^{2}}{\left(\sum_{n} e^{-\frac{\beta \hbar^{2}}{m \mu_{0}^{2}}(1-\cos n \pi \lambda)}\right)^{2}}\right]
$$

aproximando las sumas

$$
C_{V} \cong \frac{k \mathcal{N}}{2}\left[1+\pi \frac{\mu_{0}^{2}}{x^{2}}+\frac{\pi^{2} 9}{8} \frac{\mu_{0}^{4}}{x^{4}}+\ldots\right]
$$

En todas las magnitudes termodinámicas anteriores, el término principal corresponde a las variables termodinámicas de costumbre en el continuo.

Este tercer análisis para obtener las cantidades termodinámicas, aunque al final presenta expresiones aproximadas, parte de considerar formas cerradas para todas las cantidades termodinámicas, en particular de la función de partición. Sin embargo, como se vio en la sección 5.3.2, las aproximaciones son validas únicamente hasta cierto valor de $n \lesssim \lambda^{-1}$. Como se comentó antes en esta cuantización parece necesario 
incluir un corte en la energía, por ejemplo un regulador para renormalizar [166]. De esta forma podríamos preguntarnos sobre la convergencia de las sumas en la función de partición para estos valores de $n$.

Fijemos nuestra atención en la forma de la función de partición correspondiente al espectro completo (5.80), es fácil ver que puede reescribirse como

$$
Z(\beta)=e^{-\frac{x^{2}}{2 \pi \mu_{0}^{2}}} \sum_{n=0}^{\infty} \exp \left(\frac{x^{2}}{2 \pi \mu_{0}^{2}} \cos n \pi \lambda\right) .
$$

La exponencial en el argumento de la suma puede escribirse convenientemente como una suma infinita de funciones de Bessel modificadas de primer orden $I_{k}$, utilizando (C.4), [68]

$$
e^{\frac{x^{2}}{2 \pi \mu_{0}^{2}} \cos n \pi \lambda}=I_{0}\left(\frac{x^{2}}{2 \pi \mu_{0}^{2}}\right)+2 \sum_{k=1}^{\infty} I_{k}\left(\frac{x^{2}}{2 \pi \mu_{0}^{2}}\right) \cos (k n \pi \lambda) .
$$

Entonces podemos sustituir (5.91) en (5.90) y tratar de realizar la suma en $n$. Sin embargo, inmediatamente vemos que el primer término diverge. Es necesario entonces considerar la suma hasta $1 / \lambda$, como dicta la aproximación. Además cuando $\lambda \rightarrow 0$, el límite de la suma tiende a $\infty$, como en el caso continuo. Es decir, usamos $\lambda$ como regulador. Así, el resultado de la suma en (5.90) es

$$
\sum_{n=0}^{1 / \lambda} \exp \left(\frac{x^{2}}{2 \pi \mu_{0}^{2}} \cos n \pi \lambda\right)=\left(1+\frac{1}{\lambda}\right) I_{0}\left(\frac{x^{2}}{2 \pi \mu_{0}^{2}}\right)+\sum_{k=1}^{\infty} I_{k}\left(\frac{x^{2}}{2 \pi \mu_{0}^{2}}\right)\left[1+\cos k \pi+\cot \left(\frac{k \pi \lambda}{2}\right) \operatorname{sen} k \pi\right],
$$

que puede ser escrito utilizando (C.5) como

$$
\sum_{n=0}^{1 / \lambda} \exp \left(\frac{x^{2}}{2 \pi \mu_{0}^{2}} \cos n \pi \lambda\right)=\frac{1}{\lambda} I_{0}\left(\frac{x^{2}}{2 \pi \mu_{0}^{2}}\right)+\cosh \left(\frac{x^{2}}{2 \pi \mu_{0}^{2}}\right)+\sum_{k=1}^{\infty} I_{k}\left(\frac{x^{2}}{2 \pi \mu_{0}^{2}}\right) \cot \left(\frac{k \pi \lambda}{2}\right) \operatorname{sen} k \pi .
$$

La última suma es del mismo orden que el primer término ya que para $\lambda$ pequeña, $\cot k \pi \lambda / 2 \sim 2 /(k \pi \lambda)$, entonces podemos reemplazar la suma por una aproximación $\sum_{k} \operatorname{sen} k \pi / k$, que es igual a cero, para cualquier entero $k$.

De esta forma la función de partición se aproxima como:

$$
Z(\beta) \cong I_{0}\left(\frac{x^{2}}{2 \pi \mu_{0}^{2}}\right) \frac{e^{-\frac{x^{2}}{2 \pi \mu_{0}^{2}}}}{\lambda}+\frac{1}{2}\left(1+e^{-\frac{x^{2}}{\pi \mu_{0}^{2}}}\right)
$$

La expresión (5.94) es una expresión analítica para la función de partición $Z$. Por completez se calculan las cantidades termodinámicas que se obtienen con la función de partición (5.94) y son las siguientes:

$$
\begin{gathered}
F=-\frac{N}{\beta}\left[1-\ln N+\ln \left(\lambda^{-1} I_{0} \exp \left(-\frac{x^{2}}{2 \pi \mu_{0}^{2}}\right)+\frac{1}{2}\right)\right], \\
\mathcal{S}=N k\left[-\ln N+1+\ln \left(\lambda^{-1} I_{0} \exp \left(-\frac{x^{2}}{2 \pi \mu_{0}^{2}}\right)+\frac{1}{2}\right)+\frac{\hbar^{2} \beta}{m \mu_{0}^{2}}\left(\frac{I_{0}-I_{1}+\lambda e^{-\frac{x^{2}}{2 \pi \mu_{0}^{2}}}}{\left.\left.I_{0}+\lambda e^{-\frac{x^{2}}{2 \pi \mu_{0}^{2}}}\right)\right],},\right.\right.
\end{gathered}
$$




$$
\begin{gathered}
\mathcal{P}=\frac{N}{\beta L}\left[\frac{I_{0}}{\left.I_{0}+\frac{\lambda}{2} e^{-\frac{x^{2}}{2 \pi \mu_{0}^{2}}}\right],}\right. \\
U=N\left(\frac{\hbar^{2}}{m \mu_{0}^{2}}\right)\left(\frac{I_{0}-I_{1}+\lambda e^{-\frac{x^{2}}{2 \pi \mu_{0}^{2}}}}{I_{0}+\lambda e^{-\frac{x^{2}}{2 \pi \mu_{0}^{2}}}}\right), \\
C_{V}=\frac{N k}{2}\left(\frac{\hbar^{2} \beta}{m \mu_{0}^{2}}\right)^{2} \frac{I_{0}\left(I_{0}+I_{2}\right)-2 I_{1}^{2}+\lambda e^{-\frac{x^{2}}{2 \pi \mu_{0}^{2}}}\left(I_{0}+2 I_{1}+I_{2}\right)}{\left(I_{0}+\lambda e^{-\frac{x^{2}}{2 \pi \mu_{0}^{2}}}\right)^{2}} .
\end{gathered}
$$

donde se omitió el argumento de las funciones de Bessel modificadas por no cargar demasiado la notación. Es interesante notar que, como $1 / \mu_{0}^{2}$ es muy grande, podemos considerar el desarrollo asintótico de $I_{0}$ dado por (C.11), con lo que se obtiene:

$$
Z(\beta) \approx \frac{L}{x}\left[1+\frac{\pi}{4} \frac{\mu_{0}^{2}}{x^{2}}+\frac{9 \pi^{2}}{32} \frac{\mu_{0}^{4}}{x^{4}}+\frac{75 \pi^{3}}{128} \frac{\mu_{0}^{6}}{x^{6}}+\frac{3675 \pi^{4}}{2048} \frac{\mu_{0}^{8}}{x^{8}}+\ldots\right]+\frac{1}{2}\left(1+e^{-\frac{x^{2}}{\pi \mu_{0}^{2}}}\right)
$$

que es exactamente la misma serie que en (5.77), pero con un par de términos adicionales. Con esta misma aproximación, la presión sufre otro tipo de modificaciones que no son tan directas de interpretar como en el enfoque anterior

$$
\mathcal{P}=\frac{N}{\beta L}\left[1 /\left(1+\frac{\mu_{0}}{2 L} \frac{1}{1-\frac{\pi \mu_{0}^{2}}{4 x^{2}}+\frac{11 \pi^{2} \mu_{0}^{4}}{32 x^{4}}+\ldots}\right)\right],
$$

pero que siguen siendo pequeños.

Es fácil ver que el término $\exp \left(-\frac{x^{2}}{\pi \mu_{0}^{2}}\right)$ tiende a cero como $\mu_{0} \rightarrow 0$, por otra parte, el término $1 / 2$ es comparable con el término principal de la serie $L / x$, se trata de un término no despreciable que no es una corrección a la función de partición debida a la cuantización por lazos y por lo tanto a las variables termodinámicas. Este término proviene de la forma en que se aproximó la suma, podemos darnos cuenta de ello utilizando la aproximación de Euler-Maclaurin para calcular la suma, este cálculo es usual en mecánica estadística estándar

$$
\sum_{n=0}^{N} f(n) \cong \int_{0}^{N} f(n) d n+\frac{1}{2}(f(0)+f(N))+\ldots
$$

Si aproximamos directamente con esta fórmula la suma en (5.90) usando la misma regularización $1 / \lambda$

$$
\sum_{n=0}^{1 / \lambda} \exp \left(\frac{x^{2}}{2 \pi \mu_{0}^{2}} \cos n \pi \lambda\right) \cong \int_{0}^{1 / \lambda} \exp \left(\frac{x^{2}}{2 \pi \mu_{0}^{2}} \cos n \pi \lambda\right) d n+\frac{1}{2}\left[\exp \left(\frac{x^{2}}{2 \pi \mu_{0}^{2}}\right)+\exp \left(-\frac{x^{2}}{2 \pi \mu_{0}^{2}}\right)\right]+\ldots
$$


obtenemos

$$
\sum_{n=0}^{1 / \lambda} \exp \left(\frac{x^{2}}{2 \pi \mu_{0}^{2}} \cos n \pi \lambda\right) \cong \frac{1}{\lambda} I_{0}\left(\frac{x^{2}}{2 \pi \mu_{0}^{2}}\right)+\cosh \left(\frac{x^{2}}{2 \pi \mu_{0}^{2}}\right)+\ldots,
$$

lo cual lleva exactamente a la misma función de partición como en (5.94) y por lo tanto a la aproximación (5.100). El término 1/2 se debe únicamente a la forma en que se aproxima la suma. Ciertamente aparece también cuando aproximamos la función de partición de un gas ideal sin correcciones poliméricas, mediante la serie de Euler-Maclaurin:

$$
Z_{E M}=\frac{L}{x}+\frac{1}{2}+\ldots
$$

Sin embargo, si utilizamos la fórmula de Poisson tenemos

$$
Z_{P}=\frac{L}{x}\left(1+e^{-4 \pi \frac{L^{2}}{x^{2}}}+\ldots\right)
$$

En esta aproximación la primera corrección es muy pequeña en comparación con el término principal, esto nos da control sobre la serie, ya que para tener términos pequeños en el caso (5.105) necesitamos considerar muchos más términos. Por ello, esta última aproximación parece ser mejor.

Existe otra posibilidad para el gas ideal, que es considerar la suma total la cual podemos identificar con la función $\Theta_{3}$ elíptica de Jacobi [68]:

$$
Z_{T o t}=\frac{1}{2}\left[1+\Theta_{3}\left(0, e^{-\frac{\pi}{4} \frac{x^{2}}{L^{2}}}\right)\right] .
$$

Al comparar el comportamiento de (5.105)-(5.107) para el gas ideal, nos damos cuenta que para temperaturas grandes todas las ecuaciones coinciden con la función de partición clásica $L / x$. A medida que disminuye la temperatura, la aproximación de Euler-Maclaurin está más cerca de $z_{\text {Tot }}$, mientras que la aproximación de Poisson está más cerca del comportamiento clásico.

Como buscamos posibles efectos inducidos por la escala de longitud mínima sobre el comportamiento clásico, preferimos utilizar la aproximación de Poisson (5.106), que nos da un control más sistemático de la serie. A bajas temperaturas el comportamiento cuántico se vuelve más importante, sin embargo ninguna de las ecuaciones (5.105) o (5.107), nos da una expresión del todo correcta porque no se consideró el factor de la degeneración de fermiones o bosones. Como se dijo en la sección previa, sería interesante extender este análisis al caso de las estadísticas cuánticas.

\subsection{Discusión}

La mecánica cuántica "polimérica" es un modelo que incluye algunos aspectos matemáticos que asemejan a la gravedad cuántica de lazos, en particular la estructura discreta del espacio. Toma su nombre de los gráficos encajados en el espacio que proveen un espectro discreto para los operadores geométricos como el de área y el de volumen; estos gráficos asemejan estructuras poliméricas. 
El objetivo de este capítulo fue el de explorar posibles efectos que la cuantización por lazos puede tener en sistemas simples unidimensionales. Usando los espectros de energía provenientes de esta cuantización, obtuvimos la función de partición canónica que da lugar a cantidades termodinámicas modificadas por la naturaleza discreta de este tipo de cuantización no estándar. Particularmente estas cantidades se modifican por términos que contienen al parámetro $\mu_{0}$. Encontramos que la termodinámica estándar de estos sistemas simples está contenida en el límite de $\mu_{0} \rightarrow 0$ de nuestros resultados.

Hay por lo menos dos interpretaciones posibles para $\mu_{0}$ : Por un lado $\mu_{0}$ puede ser una longitud fundamental, como la longitud de Planck, en cuyo caso los efectos resultantes serían muy pequeños [165]. También se ha interpretado como un regulador para llevar a cabo un procedimiento de renormalización, cuyo límite continuo resulta ser la habitual mecánica cuántica de Schrödinger [166]. En este contexto sería interesante aplicar el procedimiento de renormalización a nivel macroscópico en estos sistemas simples, por ejemplo, en analogía con el modelos de Ising. Se puede pensar en aplicar el método seguido en [166, 167] a un modelo cuantizado por lazos con interacciones. Esto podría conducir a un flujo de renormalización no trivial para las constantes de acoplamiento y de la posible existencia de un punto fijo en el límite continuo en el que podemos comparar con la formulación usual de Schrödinger de la mecánica cuántica.

Se muestra que el espectro de una partícula en una dimensión, es similar a aquel obtenido a partir de en un principio de incertidumbre generalizado [185]. Esto no es sorprendente, ya que la misma cuantización polimérica conduce a modificaciones similares a las de GUP. En [165], Ashtekar et al. calcularon la relación de incertidumbre para los valores de expectación de $\hat{x}$ y $\hat{K}$ definido en (5.11) que se utiliza en lugar del operador de momento que no está bien definido. Los valores esperados se calculan utilizando las sombras ${ }^{15}$ de los estados semiclásicos coherentes poliméricos, la relación de incertidumbre obtenida es (5.21), que puede expresarse como:

$$
\Delta x \Delta K=\frac{\hbar}{2}\left(1-\frac{\lambda^{2}}{4}+\mathcal{O}\left(\lambda^{4}\right)\right)
$$

En este caso, la primera corrección de la cuantización por lazos es de orden $\lambda^{2}$ y disminuye la incertidumbre. La elección usual en la fenomenología de gravedad cuántica es simplemente sumar un término positivo que aumenta la incertidumbre $\Delta x \Delta p \approx \hbar\left(1+\ell_{\text {min }}^{2} \Delta p^{2} / \hbar^{2}\right) / 2$. Entonces, con este modelo de cuantización por lazos se pueden reproducir algunos resultados que en la literatura de la fenomenología de gravedad cuántica se colocaron a mano. En este modelo se derivaron de forma natural bajo el supuesto de una estructura discreta del espacio.

Estos efectos de la estructura discreta, pueden interpretarse como una modificación en el número de microestados accesibles del sistema como en [193], donde hay una redefinición de la constante de Planck suponiendo una escala mínima de longitud $\ell_{\text {min }}$. En ese trabajo se encontró que el volumen mínimo cambia a $h_{G U P}=h\left(1+\ell_{\min }^{2} p^{2} / \hbar^{2}\right)$. Nos damos cuenta de que en el caso cuantizado por lazos, (5.108), podríamos tener la misma interpretación redefiniendo la constante de Planck.

\footnotetext{
${ }^{15}$ Los estados sombra son las proyecciones de los estados físicos en el espacio pre-Hilbert dual $C y l^{*}$, sobre el conjunto de las funciones cilíndricas de una gráfica fija $C y l_{\gamma}$.
} 
Este cambio en el número de microestados es razón suficiente para preguntarse acerca de cómo la mecánica estadística de estos sistemas cambiaría. En particular, en [193] se calcula la entropía de un gas ideal con esta modificación. Nuestros resultados son comparables a los de [193], en el sentido de que las correcciones sobre la entropía son del mismo orden que en la escala de longitud mínima.

En la literatura encontramos varios casos en que la termodinámica y mecánica estadística de los sistemas se estudian mediante modificaciones a la teoría fundamental subyacente basados en las mismas ideas de la fenomenología la gravedad cuántica. Por ejemplo, en [194], las correcciones se deben a la ruptura de la invariancia Lorentz en una extensión del modelo estándar (SME) [195], [196], no hay cambio en la ecuación de estado del gas ideal, como en nuestro caso. Ambos, el potencial químico y la ecuación de Sackur-Tetrode para la entropía se modifican por términos que violan la simetría de Lorentz y, como se argumenta, estos términos pueden ser moldeados en una redefinición de la masa efectiva de las partículas. En nuestro caso tenemos cualitativamente cambios similares, aunque no tratamos con la invariancia relativista de Lorentz, la simetría de Galileo correspondiente se ha estudiado en [171], ahí se ve claramente que la simetría inherente a este modelo polimérico no es la de Galileo sino que ésta es sólo una aproximación.

Por ejemplo, en el marco de la fenomenología de la gravedad cuántica se ha estudiado la termodinámica de algunos sistemas como la radiación del cuerpo negro o un gas relativista en las etapas tempranas del universo. Estos estudios muestran que las magnitudes termodinámicas son modificadas por los términos que dependen de la escala de longitud mínima. En [189] y [190] se exploran las correcciones a las cantidades termodinámicas de la radiación del cuerpo negro, debido a la gravedad cuántica, por medio de una relación de dispersión modificada. En el caso de [189] la modificación de la relación de dispersión se obtiene una aproximación semiclásica de la gravedad cuántica de lazos, y en [190] de la teoría de cuerdas y otras propuestas alternativas. En ambos casos las correcciones son de la siguiente forma genérica

$$
\mathcal{A}=\mathcal{A}_{0}\left[1+\alpha_{1} \ell_{\min }^{2} T^{2}+\alpha_{2} \ell_{\min }^{4} T^{4}+\ldots\right]
$$

donde $\mathcal{A}$ son las funciones termodinámicas modificadas, $\mathcal{A}_{0}$ representan las cantidades sin modificación, $\alpha_{1}$ and $\alpha_{2}$ son algunos coeficientes numéricos que difieren en función de la propuesta de que la gravedad cuántica que se considere (en [189], $\alpha_{2}=0$ ). En ambos casos, la dependencia de la temperatura es la misma y el término principal es de orden $\mathcal{O}\left(\ell_{\text {min }}^{2}\right)$ similar a los resultados presentados aquí. Incluso en el caso de la termodinámica de un gas relativista en el universo primitivo, que se estudió en [197], se encuentran dependencias similares en $\ell_{\min }$ y $T$.

En contraste con nuestro análisis de la radiación de cuerpo negro donde genéricamente no encontramos dependencia en la temperatura para las modificaciones poliméricas sino únicamente en la escala de longitud mínima los términos dominantes en las correcciones son de orden $\lambda^{2}$, similar a la mayoría de los casos estudiados en la fenomenología de la gravedad cuántica.

Un análisis alternativo puede encontrarse en [191]. En dicho trabajo el efecto de tener una longitud mínima en las propiedades termodinámicas de un gas de fotones a partir de las relaciones de dispersión 
modificadas, se interpreta como una interacción repulsiva entre los fotones, similar a lo que sucede en el gas de Van der Waals. En el tratamiento aquí presentado, la ecuación de estado no se ve afectada, esto no nos permite dar una interpretación similar de nuestros resultados. Sin embargo, como se anunció antes hace falta un análisis de ensambles y funciones de partición más generales.

Como paso siguiente sería interesante explorar las modificaciones en la función de partición canónica debido a la discretización del espacio. Está claro que habrá cambios en el número de estados disponibles en el sistema, esto cambiaría el conteo de dichos estados que lleva a la obtención de la función de partición. El conteo correcto de estos estados llevará a las estadísticas correspondientes de sistemas en este esquema de cuantización. Esto tendrá consecuencias sobre las propiedades de los sistemas, por ejemplo a través del teorema de espín-estadística [187]. Un indicio de las posibles modificaciones que se tendrían, por ejemplo en las estadísticas cuánticas es la expresión encontrada para el número de ocupación (5.60). Sin embargo, es necesario primero extender el análisis y realizar conteos para otros ensambles, como el gran canónico, así como entender el rol del corte en la energía en la aproximaciones a las sumas que definen la función de partición. Una pista de las modificaciones esperadas puede dárnosla la distribución de cuerpo negro (5.60), donde claramente se ven modificaciones debidas a la cuantización por lazos. Siguiendo un procedimiento semejante a [189] podría estudiarse la correspondiente función de distribución gran canónica.

Un ejemplo en relación con los estados de la gravedad de cuántica de lazos es el modelo denominado quantum graphity, donde los estados de un sistema corresponden a gráficos que simulan de estados de red de espín [198]. El Hamiltoniano de este modelo depende de las propiedades de los gráficos y la función de partición correspondiente tendrá, en general, diferentes contribuciones [199]. La estadística de este modelo es similar a la estadística de los nudos [200], donde también se modifica la función de partición.

Existen funciones de partición generalizadas, por ejemplo, para sistemas discretos no lineales [201] o sistemas no-extensivos [202]. Tomemos como ejemplo la entropía encontrada aquí (5.73); se puede demostrar que es una cantidad extensiva cuando aproximamos el logaritmo para valores pequeños. Podemos comprobar que en realidad es una función homogénea, sin embargo, esto no se mantiene más allá de esta aproximación, y tal vez una opción sería explorar, para órdenes superiores en la aproximación, las generalizaciones de estadísticas no extensivas.

Otra posibilidad es analizar la cuantización por lazos en redes irregulares en busca de diferencias con el modelo actual. 
5.5. Discusión 


\section{Conclusiones y Perspectivas}

En este capítulo se resumen los resultados de los distintos capítulos del presente trabajo de tesis, y se discuten algunas posibles extensiones.

El interés en combinar los principios de la relatividad con los de la física estadística ha retomado ímpetu debido a las observaciones y experimentos recientes como por ejemplo en la física de altas energías [34], en astrofísica [24] y cosmología [67]. Esta combinación también es importante en la discusión de los fundamentos teóricos necesarios en la descripción de sistemas relativistas de muchos cuerpos [36], su formulación generalmente covariante [37], la termodinámica de hoyos negros [39] e incluso propuestas de gravedad cuántica [41], [42] y teoría de cuerdas [43].

En esta tesis estudiamos los siguientes sistemas: un gas simple relativista, mezclas semirelativistas cercanas al equilibrio y sistemas simples cuantizados por lazos, utilizando los métodos de la física estadística.

En primer lugar, en el capítulo 3, se estudió el gas simple relativista. Se obtuvo una expresión (3.41) de la función de distribución del equilibrio para este gas, basándose en un análisis manifiestamente covariante de la ecuación de Boltzmann. En este, no se supone un carácter específico bajo transformaciones de Lorentz para la temperatura. Notablemente la temperatura resulta estar relacionada con la norma $\Theta$, de un vector de Lorentz $\Theta^{\mu}$ (3.30), es decir, es la temperatura determinada por un observador en un marco comóvil con el gas. Tal temperatura es invariante de la misma forma que lo es la masa en reposo de una partícula puntual. Utilizando estos resultados se encontró además una versión manifiestamente covariante del teorema de equipartición, dado por la ecuación (3.66). Esta se obtiene proyectando el momento relativista del gas en la dirección del vector térmico $\Theta^{\mu}$. Este resultado coincide con versiones reportadas previamente en la literatura [70,78], pero que no son manifiestamente covariantes.

Notablemente el uso del vector térmico es consistente con la descripción del fondo cósmico de radiación (CMB). Aunque el análisis estándar de este fondo alude a una temperatura anisotrópica determinada 
por un marco en movimiento respecto a tal fondo, en realidad las observaciones aportan una distribución anisotrópica de energía de la radiación y no una medición directa de la temperatura anisotrópica. Por otro lado, el uso del vector térmico permite describir a la radiación produciendo directamente una distribución angular de energía. Más aún, la extrapolación del análisis estándar del fondo de radiación al caso de partículas masivas (por ejemplo, un fondo de neutrinos (CNB)), lleva a una temperatura anisotrópica físicamente inaceptable, pues depende del momento individual de las partículas.

Cabe mencionar que la identificación de la norma del vector térmico con el recíproco de la temperatura comóvil al gas, surge de suponer válida la termodinámica clásica en tal marco comóvil. En particular, las cantidades termodinámicas que aquí se utilizan están definidas como integrales sobre hipersuperficies tipo tiempo. Sería interesante elaborar sobre esta forma de implementar la termodinámica relativista y contrastarla con las varias versiones de ésta existentes en la literatura [72, 83, 71, 103, 104].

En los análisis existentes del caso de un gas relativista fuera de equilibrio [12, 13], es decir, cuando en la ecuación de Boltzmann el término de colisión no es nulo, usualmente se utiliza la representación de la velocidad hidrodinámica espacial y la temperatura [18] para implementar el método de ChapmanEnskog [129]. En vista de que el vector térmico contiene tanto a la temperatura comóvil, como a las componentes de la velocidad hidrodinámica, sería interesante explorar la correspondiente hidrodinámica fuera de equilibrio en la representación de $\Theta^{\mu}$; incluso, esta descripción permitiría una interpretación más completa del vector térmico. Lo mismo podría hacerse en el caso de un espacio-tiempo curvo [10, 11, 206]. El vector térmico se ha utilizado también en el análisis de la física estadística de sistemas covariantes generales [37], interpretándose sus componentes como los parámetros necesarios para describir el equilibrio del sistema cuando no se ha especificado la variable que se utilizará como variable temporal. Este análisis podría extenderse también al caso fuera de equilibrio.

Puede intentarse extender este análisis estadístico covariante para encontrar posibles efectos a nivel termodinámico en ciertas teorías alternativas a la relatividad especial. Estas se han propuesto para resolver ciertos problemas que tienen su origen en la gravitación cuántica, e incluyen aquellas donde la velocidad de la luz se considera variable (VSL) [110], deformaciones del álgebra de Poincaré (DSR) [111].

En el capítulo 4, se estudiaron mezclas binarias con una componente relativista y otra no relativista. El término de colisión de la correspondiente ecuación de Boltzmann que gobierna la evolución de la distribución del sistema, pudo aproximarse por un término diferencial tipo Fokker-Planck. Esto se logro dadas las características de las partículas de cada componente de la mezcla, como su densidad, masa y energía. Particularmente, en el caso del gas de Rayleigh o movimiento Browniano relativista, se considera un gas de fondo formado por partículas relativistas, en donde se difunden partículas pesadas no relativistas es decir la densidad de las partículas del gas es mucho mayor que el de las partículas brownianas, mientras que la masa de las partículas de gas es mucho menor que el de las brownianas. La ecuación de FokkerPlanck (4.20) permite identificar los coeficientes de difusión y fricción viscosidad (4.22). Los casos extremos de estos coeficientes se calcularon, en particular límite no relativista (4.23) coincide con los resultados 
clásicos de Wang Chang y Uhlenbeck y de Green [119, 120].

El caso del gas de Lorentz, puede interpretarse físicamente como complementario al gas de Rayleigh debido a que las características se invierten, es decir, ahora el gas está formado por partículas no relativistas cuya masa es mayor a las de las partículas que se difunden, que son relativistas y de masa pequeña. La ecuación de Fokker-Planck (4.33) contiene tres términos cuyos coeficientes no son constantes y pueden asociarse respectivamente al tensor de difusión, al vector de fricción dinámica y a un tiempo característico de relajación. Términos semejantes a este último han sido utilizados en la literatura para modelar tasas de creación, de crecimiento o reacción [143, 132], o bien describir efectos de retardo o memoria [144].

Por otra parte, mostramos que el gas relativista de una componente en la aproximación de colisiones rasantes, admite una descripción a través de una ecuación de Fokker-Planck covariante. Este análisis resultó al utilizar desviaciones de la distribución manifiestamente covariante (3.41) obtenida en el capítulo 3. Sería interesante extender el análisis covariante al caso de las mezclas.

En ciertos enfoques de la gravedad cuántica se plantea la búsqueda de efectos provenientes de una escala de longitud mínima. Dada la existencia de esta longitud se propone sustituir las trayectorias de las partículas libres por procesos difusivos en el espacio-tiempo [41], o bien plantear una ecuación estocástica para modelar dichos posibles efectos, suponiendo fluctuante la métrica del espacio tiempo [42]. Sería entonces interesante obtener ecuaciones tipo Fokker-Planck que modelen estos efectos pero que provengan de una teoría microscópica de la gravedad que podrían justificar algunos análisis fenomenológicos.

Motivados por las posibles aplicaciones de la física estadística a diversas propuestas de gravedad cuántica, finalmente en el capítulo 5 exploramos posibles efectos que una escala de longitud mínima puede arrojar en un análisis termoestadístico de sistemas simples cuantizados con un esquema que implementa la gravedad cuántica de lazos. El esquema de cuantización no resulta equivalente a la cuantización usual de Schrödinger en mecánica cuántica. Estudiamos entonces las modificaciones que esta longitud mínima induce en las correspondientes cantidades termodinámicas obtenidas del análisis mecánico estadístico usual. Hay por lo menos dos interpretaciones posibles para este parámetro. Puede considerarse como la longitud de Planck. En este caso los efectos resultantes serían pequeños. Alternativamente se ha considerado como un regulador para realizar un procedimiento de renormalización, cuyo límite continuo resulta ser la habitual mecánica cuántica de Schrödinger [166, 167, 205].

Estudiamos dos sistemas simples: El oscilador armónico y una partícula confinada en una caja. Ensambles estadísticos del primer sistema modelarán por una parte, vibraciones en una variante de un sólido tipo Einstein, y por otra modificaciones a la radiación de cuerpo negro; mientras que el segundo modelará un tipo de gas ideal. Las cantidades termodinámicas obtenidas se ven modificadas por el parámetro de longitud, y se asemejan a aquellas obtenidas en la literatura que sigue un esquema fenomenológico de la gravedad cuántica [173] y a ciertas propuestas de generalizaciones de la relación de incertidumbre [197, 185]. Todas las correcciones se anulan cuando el parámetro de longitud se hace cero, reobteniéndose la termodinámica usual de los sistemas correspondientes. 
En este trabajo se utiliza la función de partición canónica de la estadística de Maxwell-Boltzmann por ser la más simple. La relación entre la estructura discreta y la correspondiente modificación a la estadística no fue tratado y es un problema interesante que continúa abierto. Es evidente que habrá cambios en el número de microestados disponibles en el sistema, esto tendría consecuencias sobre las propiedades de los sistemas, por ejemplo a través del Teorema de espín-estadística y quizá lleve a posibilidades exóticas [187]. Un indicio de estas modificaciones es el número de ocupación modificado obtenido en (5.60), esto muestra que quizá al realizar el conteo de estados considerando la discretez del espacio y la simetría de las funciones de onda, existan modificaciones en las funciones de partición y por tanto en los ensambles correspondientes.

Así como se ha implementado el límite continuo de la teoría cuántica a través de un procedimiento de renormalización, sería igualmente interesante implementar un procedimiento similar a nivel macroscópico, es decir, en las variables termodinámicas de manera semejante a como se estudia el modelo de Ising, y verificar que la escala introducida también es removida a ese nivel.

Otro aspecto interesante sería estudiar las propiedades de invariancia de Lorentz en estos sistemas cuantizados por lazos. Se ha visto que es posible reconciliar la invariancia de Lorentz con la geometría espacial discreta [203, 204], en contraste con ciertos enfoques fenomenológicos que introducen violaciones a la simetría de Lorentz como MDR y GUP [173, 174].

Por otra parte la estructura discreta de la gravedad cuántica de lazos sugiere preguntarnos ¿De qué forma se recupera la relatividad general clásica a partir de la gravedad cuántica de lazos? ¿Es posible recobrar, en un sentido aproximado, un espacio-tiempo continuo desde un conjunto discreto?

Una manera de abordar el problema es a través de una analogía con el movimiento browniano de una partícula en un medio. Las ecuaciones de movimiento se ven afectadas por un término aleatorio que toma en cuenta la irregularidad de las colisiones de la partícula con los átomos del medio [122, 126]. Para la gravedad cuántica el movimiento browniano de una partícula propagándose por el espacio-tiempo podría revelar su microestructura. Como ya se mencionó, esta idea ya se ha explorado en la literatura en propuestas como la gravedad estocástica [42] que supone la posible existencia de fluctuaciones de la geometría del espacio-tiempo debidas a efectos cuánticos, que se codifican en un término estocástico en las ecuaciones de la relatividad general. A partir de esta modificación se ha encontrado una ecuación para la difusión en el espacio-tiempo fluctuante. Este es un ejemplo de posibles efectos cuánticos gravitacionales. Los enfoques a la gravedad cuántica pueden clasificarse dependiendo del punto de partida del estudio, que puede ser una teoría efectiva o una teoría microscópica. Hay dos posibilidades: El enfoque descendente (top-down) donde se consideran desviaciones de las teorías clásicas provocadas por efectos cuánticos gravitacionales, modelando la microestructura subyacente por términos aleatorios, como en la gravedad estocástica. El enfoque ascendente (bottom-up) considera que la teoría macroscópica continua emerge de algún tipo de límite o promedio de las interacciones microscópicas. En esta perspectiva las teorías microscópicas como la gravedad cuántica de lazos deben contener en el límite continuo a la relatividad 
general.

En hidrodinámica existe una situación análoga, el mejor ejemplo es de nuevo el movimiento browniano. Por un lado el enfoque que utiliza el término estocástico que modela las colisiones sería el enfoque descendente. Sólo se consideran las fluctuaciones en la trayectoria macroscópica sin saber cuál es el modelo microscópico subyacente. Por otro lado se puede considerar que las interacciones de las partículas brownianas con los constituyentes del medio están dadas por colisiones elásticas, es decir, por las leyes de Newton. Se puede dar una descripción efectiva de cómo todas las interacciones entre las moléculas del medio y las partículas brownianas dan como resultado macroscópico un movimiento browniano. Esto se logra utilizando el enfoque de la ecuación de Boltzmann [129] a partir de la cual pueden hallarse cantidades macroscópicas conociendo la dinámica subyacente sin necesidad de suposiciones aleatorias, en particular puede describirse la difusión de las partículas brownianas en el fluido [119, 120]. El caso relativista especial de este problema se estudió en [105]. Este sería el enfoque ascendente.

Una línea interesante dentro de la cuantización por lazos es estudiar estos sistemas fuera del equilibrio a partir de una ecuación de Boltzmann modificada. Como se muestra en el apéndice A, para fundamentar la dicha ecuación de Boltzmann puede comenzarse de una teoría microscópica como la teoría cuántica de campo y pasar, después de ciertas aproximaciones, a la teoría cinética relativista. Así pues podría comenzarse de una teoría cuantizada por lazos como (5.16) y definir una función de Wigner para esta teoría. Afortunadamente se ha formulado matemáticamente la función de Wigner para sistemas cuantizados por lazos [207]. De esta forma se podría obtener una ecuación de Boltzmann para esa función de Wigner de lazos. Con esto podríamos estudiar efectos similares a los que encontramos aquí debidos a una escala mínima fundamental, pero en procesos fuera del equilibrio. 
6. Conclusiones y Perspectivas 


\section{La Ecuación de Boltzmann Relativista como una aproximación a la teoría cuántica de campos}

En este apéndice se describe una aproximación a la teoría cinética relativista desde la perspectiva de la teoría cuántica de campos, basándonos principalmente en el trabajo de de Groot et al [12].

\section{A.1. Dinámica de los campos}

Consideremos un sistema de partículas sin espín de masa $m$ en el contexto de la teoría cuántica de campo. Estas están descritas por un operador de campo escalar $\phi(x)$ tal que ante transformaciones de Lorentz $\phi^{\prime}\left(x^{\prime}\right)=\phi(x)$. La dinámica del sistema para campos no interactúantes está dada por la ecuación de Klein-Gordon

$$
\left(\partial_{\mu} \partial^{\mu}+\frac{m^{2} c^{2}}{\hbar^{2}}\right) \phi(x)=0
$$

la cual puede deducirse de una densidad Lagrangiana, tal que la ecuación (A.1) puede obtenerse de ecuaciones tipo Euler-Lagrange considerando independientes a $\phi$ y su conjugado

$$
\frac{\partial \mathcal{L}}{\partial \phi^{\dagger}}-\partial_{\mu} \frac{\partial \mathcal{L}}{\partial\left(\partial_{\mu} \phi^{\dagger}\right)}+\partial_{\mu} \partial_{\nu} \frac{\partial \mathcal{L}}{\partial\left(\partial_{\mu} \partial_{\nu} \phi^{\dagger}\right)}=0
$$

donde la densidad Lagrangiana correspondiente a la ecuación de Klein-Gordon

$$
\mathcal{L}_{K G}(x)=-\frac{1}{2} \hbar c\left[\phi^{\dagger}(x) \partial_{\mu} \partial^{\mu} \phi(x)+\phi(x) \partial_{\mu} \partial^{\mu} \phi^{\dagger}(x)+2 \frac{m c^{2}}{\hbar^{2}} \phi^{\dagger}(x) \phi(x)\right] .
$$

La interacción se introducirá añadiendo al Lagrangiano un término $\mathcal{L}_{\text {Int }}$ que dependerá en general de distintos campos correspondientes a las partículas con las que interactúa

$$
\mathcal{L}(x)=\mathcal{L}_{K G}(x)+\mathcal{L}_{\text {Int }} .
$$


Si se supone que $\mathcal{L}_{\text {Int }}$ no depende de las derivadas de los operadores de campo las ecuaciones de movimiento serán de la siguiente forma

$$
\left(\partial_{\mu} \partial^{\mu}+\frac{m^{2} c^{2}}{\hbar^{2}}\right) \phi(x)=\frac{1}{\hbar c} \frac{\partial \mathcal{L}_{\text {Int }}(x)}{\partial \phi^{\dagger}(x)},
$$

y una ecuación idéntica cambiando $\phi$ por $\phi^{\dagger}$.

El Lagrangiano debe ser invariante $\mathcal{L}^{\prime}\left(\phi^{\prime}\left(x^{\prime}\right), \partial_{\mu}^{\prime} \phi^{\prime}\left(x^{\prime}\right), \ldots\right)=\mathcal{L}(\phi(x), \partial \phi(x), \ldots)$ y dejar invariante las ecuaciones de movimiento $\mathcal{L}^{\prime}\left(\phi^{\prime}\left(x^{\prime}\right), \partial_{\mu}^{\prime} \phi^{\prime}\left(x^{\prime}\right), \ldots\right)=\mathcal{L}\left(\phi^{\prime}\left(x^{\prime}\right), \partial_{\mu}^{\prime} \phi^{\prime}\left(x^{\prime}\right), \ldots\right)$. De ahi se desprenden las leyes de conservación de la densidad de corriente y del tensor de energía-momento

$$
\partial_{\mu} j^{\mu}=0, \quad \partial_{\nu} t^{\mu \nu}=0
$$

Si además se pide invariancia de $\mathcal{L}$ ante traslaciones infinitesimales y se supone también invariancia ante la transformación de norma $\phi(x) \rightarrow \phi^{\prime}(x)=e^{i \theta} \phi(x)$, entonces la densidad de corriente $j^{\mu}$ y el tensor de energía momento $t^{\mu \nu}$, toman la siguiente forma

$$
\begin{aligned}
j^{\mu} & =i c \phi^{\dagger}(x) \overleftrightarrow{\partial}^{\mu} \phi(x) \\
t^{\mu \nu} & =-\frac{1}{2} \hbar c \phi^{\dagger}(x) \overleftrightarrow{\partial^{\mu}} \overleftrightarrow{\partial}^{\nu} \phi(x)-g^{\mu \nu} \mathcal{L}(x)
\end{aligned}
$$

donde la doble flecha en $\overleftrightarrow{\partial}$ indica que hay que derivar primero a la derecha y después restar la derivada de la cantidad a la izquierda.

\section{A.2. La Función de Wigner}

Según la teoría cinética no relativista las cantidades macroscópicas se definen como promedios estadísticos o momentos estadísticos de sus homólogas microscópicas, pesados por la función de distribución correspondiente. En teoría cuántica se introduce la función de Wigner que es una función del espacio fase y puede considerarse la más simple y más cercana a una función de distribución. De esta forma los promedios macroscópicos usualmente calculados como $\langle O\rangle=\operatorname{Tr}(\rho O)$, donde $O$ es una observable y $\rho$ es la matriz de densidad, se expresarán como promedios pesados por la función de Wigner de una partícula. Para ello escribiremos las cantidades macroscópicas como sigue

$$
\begin{aligned}
J^{\mu} & =\left\langle j^{\mu}(x)\right\rangle=i c\left\langle: \phi^{\dagger}(x) \overleftrightarrow{\partial}^{\mu} \phi(x):\right\rangle, \\
T^{\mu \nu} & =\left\langle t^{\mu \nu}(x)\right\rangle=-\frac{1}{2} \hbar c\left\langle: \phi^{\dagger}(x) \overleftrightarrow{\partial}^{\mu} \overleftrightarrow{\partial}^{\nu} \phi(x):\right\rangle-g^{\mu \nu}\langle: \mathcal{L}(x):\rangle
\end{aligned}
$$

donde $: *$ : indica el orden normal de los operadores de campo. La estrategia será encontrar la función de peso para este sistema e identificarla con la función de Wigner. Para ello consideremos primero la densidad de corriente (A.9) notando que puede escribirse de la siguiente forma

$$
\begin{aligned}
\left\langle: \phi^{\dagger}(x) \overleftrightarrow{\partial}^{\mu} \phi(x):\right\rangle & =\int d^{4} y \delta^{4}(y)\left\langle: \phi^{\dagger}\left(x+\frac{1}{2} y\right) \overleftrightarrow{\partial}^{\mu} \phi\left(x-\frac{1}{2} y\right):\right\rangle \\
& =2 \int d^{4} y \frac{\partial}{\partial y_{\mu}} \delta^{4}(y)\left\langle: \phi^{\dagger}\left(x+\frac{1}{2} y\right) \phi\left(x-\frac{1}{2} y\right):\right\rangle
\end{aligned}
$$


donde se introdujo una variable auxiliar $y^{\mu}$ a través de la delta de Dirac 4-dimensional, y se realizó una integración parcial en el último término. Se puede escribir $\delta^{4}(y)$ en su representación integral

$$
\delta^{4}(y)=\frac{1}{(2 \pi \hbar)^{4}} \int d^{4} k e^{-\frac{k \cdot y}{\hbar}}
$$

de forma que la densidad de corriente se puede escribir como

$$
J^{\mu}=c \int d^{4} k k^{\mu} W(x, k),
$$

donde

$$
W(x, k) \equiv \frac{4 \pi}{(2 \pi \hbar)^{5}} \int d^{4} y e^{-\frac{k \cdot y}{\hbar}}\left\langle: \phi^{\dagger}\left(x+\frac{1}{2} y\right) \phi\left(x-\frac{1}{2} y\right):\right\rangle,
$$

que se define como la función de Wigner en la teoría cuántica relativista. Es una función real y es manifiestamente escalar. Con ayuda de esta función las cantidades macroscópicas pueden escribirse como promedios en el especio de momentos $k^{\mu}$. En el caso del tensor de energía-momento (A.10) toma la siguiente forma en términos de la función de Wigner

$$
T^{\mu \nu}=c \int d^{4} k k^{\mu} k^{\nu} W(x, k)+g^{\mu \nu}\left[\frac{c}{2} \int d^{4} k\left(m^{2} c^{2}-k^{2}+\frac{1}{4} \hbar^{2} \partial_{\alpha} \partial^{\alpha}\right) W(x, k)-\left\langle: \mathcal{L}_{\text {Int }}(x):\right\rangle\right] .
$$

Notemos que el primer término en (A.15) es precisamente el segundo momento de la distribución $W$, sin embargo hay dos términos más que están relacionados con la parte interactuante. Para sistemas diluidos que son los estudiados principalmente por la teoría cinética se piensan principalmente interacciones binarias. El término de interacción se pensará proporcional a la densidad e inversamente proporcional a la temperatura, así sí el Lagrangiano $\mathcal{L}_{\text {Int }}$ tiene una constante de acoplamiento $\lambda_{I}$, el término de interacción podrá despreciarse siempre y cuando $n \lambda_{I} / k T \ll 1$. En este régimen puede despreciarse el último término de (A.15). Además de la definición de (A.14), se sigue que $W$ sigue una ecuación de evolución

$$
\left(m^{2} c^{2}-k^{2}+\frac{1}{4} \hbar^{2} \partial_{\alpha} \partial^{\alpha}\right) W(x, k)=I_{\text {Int }}
$$

donde $I_{I n t}$ es una integral relacionada con las derivadas del Lagrangiano de interacción y es proporcional a $n \lambda_{I}$. Para despreciar este término se requiere que $n \lambda_{I} / m c^{2} \ll 1$, de forma que $I_{\text {Int }} \approx 0$. Es de esta manera en que el tensor de energía-momento se identifica con el segundo momento de la distribución, es decir, el primer término de (A.15). Nótese de (A.16) cuando $I_{\text {Int }} \approx 0$, que $k^{\mu}$ no está restringido por la capa de masa $k^{2} \neq m^{2} c^{2}$. Sin embargo, entre las condiciones que impone la aproximación estadística se tiene que para que la función de Wigner pueda considerarse una función de distribución en el sentido usual, debe variar muy lentamente a escala microscópica por ejemplo a la escala de Compton $\hbar / m c$, esta condición se puede escribir como

$$
\left(\frac{\hbar}{m c}\right)^{2}\left|\partial_{\mu} \partial^{\mu} W(x, p)\right| \ll|W(x, p)|
$$


lo que implica

$$
\left(m^{2} c^{2}-k^{2}\right) W(x, k)=0,
$$

para lo cual $k^{\mu}$ es tipo-tiempo y ahora si satisface la condición de capa de masa.

Físicamente $k^{\mu}$ puede ser de tres tipos, tipo-tiempo con componente temporal positiva, tipo-tiempo con componente temporal negativa y tipo-espacio. Las correspondientes funciones de Wigner serán $W_{+}, W_{-}$ y $W_{z}$, esta última no tiene contribución macroscópica de forma que la función de Wigner puede separarse como

$$
W(x, k)=W_{+}(x, k)+W_{-}(x, k) .
$$

$\mathrm{Al}$ estar $k^{\mu}$ restringido a la capa de masa se puede escribir

$$
W_{ \pm}(x, k)=\int \frac{d^{3} p}{p^{0}} \delta^{(4)}(k \pm p) f_{ \pm}(x, p)
$$

donde $f_{ \pm}(x, p)$ son las funciones de distribución correspondientes. La densidad de corriente y el tensor de energía-momento pueden escribirse de una forma usual en teoría cinética utilizando (A.20)

$$
\begin{aligned}
J^{\mu} & =c \int \frac{d^{4} p}{p^{0}} p^{\mu}\left[f_{+}(x, p)-f_{-}(x, p)\right], \\
T^{\mu \nu} & =c \int \frac{d^{4} p}{p^{0}} p^{\mu} p^{\nu}\left[f_{+}(x, p)-f_{-}(x, p)\right] .
\end{aligned}
$$

Si consideramos por otra parte el operador de campo en ausencia de interacciones se puede separar como

$$
\phi(x)=\phi_{+}(x)+\phi_{-}(x)
$$

donde las partes de frecuencia positiva y negativa son

$$
\begin{aligned}
& \phi_{+}(x)=2 \int d^{4} p H\left(p^{0}\right) \delta\left(p^{2}-m^{2} c^{2}\right) f_{p}(x) a(p), \\
& \phi_{-}(x)=2 \int d^{4} \bar{p} H\left(\bar{p}^{0}\right) \delta\left(\bar{p}^{2}-m^{2} c^{2}\right) f_{\bar{p}}^{*}(x) b^{\dagger}(\bar{p}),
\end{aligned}
$$

donde $H(x)$ es la función escalón unitario, además se abrevió $f_{p}(x)=\left(1 / 2(2 \pi)^{3} \hbar^{2}\right) e^{-i p \cdot x / \hbar}$ y $a(p)$ son los operadores de aniquilación de partículas y $b^{\dagger}(\bar{p})$ son los de creación de antipartículas. Al sustituir las expresiones anteriores en la definición de la función de Wigner (A.14) y recordando (A.20) para la capa de masa, las funciones $F_{ \pm}$pueden escribirse de la siguiente manera

$$
\begin{aligned}
& f_{+}(x, p)=\frac{1}{(2 \pi \hbar)^{3}} \int d^{4} q \delta^{(4)}(p \cdot q) e^{-i \frac{q \cdot x}{\hbar}}\left\langle a^{\dagger}\left(p-\frac{1}{2} q\right) a\left(p+\frac{1}{2} q\right)\right\rangle \\
& f_{-}(x, p)=\frac{1}{(2 \pi \hbar)^{3}} \int d^{4} q \delta^{(4)}(p \cdot q) e^{-i \frac{q \cdot x}{\hbar}}\left\langle b^{\dagger}\left(p-\frac{1}{2} q\right) b\left(p+\frac{1}{2} q\right)\right\rangle .
\end{aligned}
$$

es interesante que el sistema puede tratarse como una mezcla de bosones $F_{+}$y antibosones $F_{-}$escalares. 


\section{A.3. Ecuación de Transporte Relativista}

Utilizando la ecuación de campo (A.5) se puede derivar una ecuación de evolución para la función de Wigner (A.14)

$k^{\mu} \partial_{\mu} W_{+}(x, k)=\frac{i}{(2 \pi \hbar)^{4}} H\left(k^{0}\right) H\left(k^{2}\right) \int d^{4} y e^{-i \frac{k \cdot y}{\hbar}}\left\langle\phi^{\dagger}\left(x+\frac{1}{2} y\right) \varrho\left(x-\frac{1}{2} y\right)-\varrho^{\dagger}\left(x+\frac{1}{2} y\right) \phi\left(x-\frac{1}{2} y\right)\right\rangle$,

donde $\varrho(x) \equiv \frac{1}{\hbar c} \frac{\partial \mathcal{L}_{I n t}}{\partial \phi^{\dagger}}$, además de una ecuación similar para $W_{-}(x, k)$. En general el Lagrangiano de interacción puede ser tal que el lado derecho de (A.28) no pueda expresarse en términos sólo de la distribución de una partícula $W_{+}$(ó $W_{-}$). Entonces en realidad la ecuación (A.28) es la primera ecuación de un sistema de ecuaciones acopladas para las funciones de distribución de dos, tres etc. partículas. Este sistema se conoce como la jerarquía BBGKY (por las siglas Bogoliubov, Born Green, Kirwood, Yvon). Para obtener la ecuación cinética (tipo Boltzmann) es necesario introducir una suposición conocida como la hipótesis del caos molecular, la cual establece que cualquier correlación inicial entre las partículas será despreciada.

Para establecer la condición inicial es necesario escribir primero los promedios estadísticos de cualquier variable en términos de cantidades que serán útiles. En la teoría cuántica de campos el espacio de Hilbert en el que actúan los operadores de campo es generado por el vacío $|0\rangle$ y todos los estados de partículas y antipartículas entrantes (in-states) $\left|p_{n}, \bar{p}_{m}\right\rangle_{i n}$, con $n, m=1,2, \ldots$, (alternativamente pueden usarse los estados salientes etiquetados por el subíndice out, out-states). Utilizando sólo estados de energía positiva se tiene

$$
\left|p_{n}\right\rangle_{i n}=a_{i n}^{\dagger}\left(p_{n}\right)|0\rangle,
$$

donde $a_{i n}^{\dagger}\left(p_{n}\right)$ es el producto de los operadores de creación de los estados entrantes para cada momento de $n=1,2, \ldots$

Considerando un operador arbitrario $O$, se puede demostrar [12], que su promedio estadístico $\langle O\rangle$, puede escribirse primero en términos de las matrices de densidad reducidas de $n$-partículas, cuyas entradas se interpretan como las probabilidades de encontrar a $n$ partículas con momento dado en el estado inicial y se escribe como sigue

$$
\left\langle a_{i n}^{\dagger}\left(p_{n}\right) a_{i n}\left(p_{n}^{\prime}\right)\right\rangle=\sum_{k=0}^{\infty} \frac{1}{k !} \int \frac{d^{3} p_{k}}{p_{k}^{0}}{ }_{i n}\left\langle p_{k}, p^{\prime}{ }^{n}|\rho| p_{k}, p_{n}\right\rangle_{i n} .
$$

Utilizando una relación de completes y la definición del promedio y (A.30), puede escribirse $\langle O\rangle$ en términos de las matrices reducidas, sin embargo, como lo operadores de campo entrantes pueden escribirse en términos de los operadores $a(p)_{i n}$, (A.24), resulta conveniente expresar el promedio de $O$ en términos de los operadores de campo, finalmente

$$
\langle O\rangle=\sum_{n=0}^{\infty} \frac{1}{(n !)^{2}} \int d^{4} x_{n} d^{4} x_{n}^{\prime} \tilde{O}_{n}\left(x_{n} ; x_{n}^{\prime}\right)\left\langle\phi_{+\left({ }_{i n}\right)}^{\dagger}\left(x_{n}\right) \phi_{+\left({ }_{i n}\right)}\left(x_{n}^{\prime}\right)\right\rangle,
$$


donde

$$
\tilde{O}_{n}\left(x_{n} ; x_{n}^{\prime}\right)=\frac{(4 \pi)^{n}}{(2 \pi \hbar)^{6 n}} \int d^{4} p_{n} d^{4} p_{n}^{\prime} \prod_{j=1}^{n} e^{-i \frac{\left(p_{j} \cdot x_{j}-p_{j}^{\prime} \cdot x_{j}^{\prime}\right)}{\hbar}}\left\{p_{n}|O| p_{n}^{\prime}\right\}
$$

y los coeficientes

$$
\left\{p_{n}|O| p_{n}^{\prime}\right\} \equiv \sum_{m=0}^{n}(-1)^{m}\left(\begin{array}{c}
n \\
m
\end{array}\right){ }_{i n}\left\langle p_{m} \mid p_{m}^{\prime}\right\rangle_{\text {in in }}\left\langle p_{n-m}|O| p_{n-m}^{\prime}\right\rangle_{i n} .
$$

La condición de que los estados iniciales no estén correlacionados puede expresarse de la siguiente forma en términos de la matriz reducida

$$
\left\langle a_{i n}^{\dagger}\left(p_{n}\right) a_{i n}\left(p_{m}^{\prime}\right)\right\rangle=\delta_{n m} \sum_{\{\text {Perm }\}} \prod_{j=1}^{n}\left\langle a_{i n}^{\dagger}\left(p_{j}\right) a_{i n}\left(p_{j}^{\prime}\right)\right\rangle,
$$

donde la suma corre sobre todas las permutaciones de los índices de los 4-momentos. Sin correlaciones el promedio del operador $O$ se escribe de la siguiente forma

$$
\langle O\rangle=\sum_{n=0}^{\infty} \frac{1}{n !} \int d^{4} x_{n} d^{4} x_{n}^{\prime} \tilde{O}_{n}\left(x_{n} ; x_{n}^{\prime}\right) \prod_{j=1}^{n}\left\langle\phi_{+\left({ }_{i n}\right)}^{\dagger}\left(x_{j}\right) \phi_{+\left({ }_{i n}\right)}\left(x_{j}^{\prime}\right)\right\rangle .
$$

Como la función de Wigner (A.14) puede definirse para frecuencias positivas y sólo para estados entrantes, y podemos notar que es esencialmente una transformada de Fourier del promedio del producto de los operadores de campo, podemos buscar la relación inversa que sería

$$
\left\langle\phi_{+\left({ }_{i n}\right)}^{\dagger}\left(x+\frac{1}{2} y\right) \phi_{+\left({ }_{i n}\right)}\left(x-\frac{1}{2} y\right)\right\rangle=\frac{\hbar}{2} \int d^{4} k e^{i \frac{k \cdot y}{\hbar}} W_{+\left({ }_{i n}\right)}(x, k),
$$

con lo cual el promedio de $O$, (A.35) puede escribirse como un promedio pesado con $W_{+\left({ }_{\text {in }}\right)}$ como la función de distribución, de la siguiente forma

$$
\langle O\rangle=\sum_{n=0}^{\infty} \frac{1}{n !} \int d^{4} x_{n} d^{4} k_{n} O_{n}\left(x_{n} ; k_{n}\right) \prod_{j=1}^{n} W_{+\left({ }_{i n}\right)}\left(x_{j}, k_{j}\right),
$$

donde el nuevo coeficiente es

$$
O_{n}\left(x_{n} ; k_{n}\right)=\frac{1}{(2 \pi \hbar)^{n}} \int d^{4} q^{n} \prod_{j=1}^{n} e^{i \frac{q_{j} \cdot x_{j}}{\hbar}}\left\{k_{n}-\frac{1}{2} q_{n}|O| k_{n}+\frac{1}{2} q_{n}\right\} .
$$

Se buscará un desarrollo en términos de las contribuciones debidas a interacciones binarias, entre tres partículas, y así sucesivamente. Para ello es útil recalcar que los operadores de campo están en la representación de Heisenberg

$$
\phi^{\dagger}\left(x+\frac{1}{2} y\right)=e^{i \frac{P \cdot x}{\hbar}} \phi^{\dagger}\left(\frac{1}{2} y\right) e^{-i \frac{P \cdot x}{\hbar}}, \quad \phi\left(x-\frac{1}{2} y\right)=e^{i \frac{P \cdot x}{\hbar}} \phi\left(-\frac{1}{2} y\right) e^{-i \frac{P \cdot x}{\hbar}},
$$

con lo que la función de Wigner puede reexpresarse de la siguiente forma

$$
W_{+}(x, k)=\left\langle e^{i \frac{P \cdot x}{\hbar}} \omega_{+}(k) e^{-i \frac{P \cdot x}{\hbar}}\right\rangle,
$$


donde

$$
\omega_{+}(k)=\frac{4 \pi}{(2 \pi \hbar)^{5}} H\left(k^{0}\right) H\left(k^{2}\right) \int d^{4} y e^{-i \frac{k \cdot y}{\hbar}} \phi^{\dagger}\left(\frac{1}{2} y\right) \phi\left(-\frac{1}{2} y\right) .
$$

Ahora bien, como los estados entrantes son eigenestados de el 4-momento total $P^{\mu}$, la expresión (A.37) puede aplicarse a la función de Wigner de los estados entrantes tal que

$$
W_{+}(x, k)=\sum_{n=0}^{\infty} \frac{1}{n !} \int d^{4} x_{n} d^{4} k_{n} \omega_{n}\left(x_{n}, p_{n} ; k\right) \prod_{j=1}^{n} W_{+\left({ }_{i n}\right)}\left(x+x_{j}, p_{j}\right),
$$

que es el desarrollo buscado. En este caso

$$
\omega_{n}\left(x_{n}, p_{n} ; k\right)=\frac{1}{(2 \pi \hbar)^{n}} \int d^{4} q^{n} \prod_{j=1}^{n} e^{i \frac{q_{j} \cdot x_{j}}{\hbar}}\left\{p_{n}-\frac{1}{2} q_{n}\left|\omega_{+}(k)\right| p_{n}+\frac{1}{2} q_{n}\right\} .
$$

Se pueden calcular los coeficientes (A.43) para cada caso, así para $n=0$ se tienen que $\omega_{0}(x, p ; k)=0$. Para $n=1$ se obtiene $\omega_{1}(x, p ; k)=\delta^{(4)}(k-p) \delta^{(4)}(x)$. Con estos resultados el desarrollo (A.42) de la función de Wigner se puede escribir como

$$
W_{+}(x, k)=W_{+\left({ }_{i n}\right)}(x, k)+\sum_{n=2}^{\infty} \frac{1}{n !} \int d^{4} x_{n} d^{4} p_{n} \omega_{n}\left(x_{n}, p_{n} ; k\right) \prod_{j=1}^{n} W_{+\left({ }_{i n}\right)}\left(x+x_{j}, p_{j}\right) .
$$

El primer término de (A.44) corresponde al caso sin interacciones ya que $k^{\mu} \partial_{\mu} W_{+\left({ }_{i n}\right)}(x, k)=0$, el término de la suma es precisamente el término que contiene las interacciones entre las partículas del sistema. Ahora bien para estudiar la evolución de esta función de Wigner es necesario regresar a la ecuación de evolución (A.28). Con el mismo razonamiento anterior, puede obtenerse un desarrollo para esta ecuación de evolución

$$
k^{\mu} \partial_{\mu} W_{+}(x, k)=\sum_{n=2}^{\infty} \frac{1}{n !} \int d^{4} x_{n} d^{4} p_{n} \Delta_{n}\left(x_{n}, p_{n} ; k\right) \prod_{j=1}^{n} W_{+\left({ }_{i n}\right)}\left(x+x_{j}, p_{j}\right),
$$

donde ahora las funciones son las siguientes

$$
\begin{aligned}
\Delta_{n}\left(x_{n}, p_{n} ; k\right) & =\frac{1}{(2 \pi \hbar)^{n}} \int d^{4} q^{n} \prod_{j=1}^{n} e^{i \frac{q_{j} \cdot x_{j}}{\hbar}}\left\{p_{n}-\frac{1}{2} q_{n}\left|\Delta_{+}(k)\right| p_{n}+\frac{1}{2} q_{n}\right\} \\
\Delta_{+}(k) & =\frac{i}{(2 \pi \hbar)^{4}} H\left(k^{0}\right) H\left(k^{2}\right) \int d^{4} y e^{-i \frac{k \cdot y}{\hbar}}\left[\phi^{\dagger}\left(\frac{1}{2} y\right) \varrho\left(-\frac{1}{2} y\right)+\varrho^{\dagger}\left(\frac{1}{2} y\right) \phi\left(-\frac{1}{2} y\right)\right]
\end{aligned}
$$

Para ambas expresiones (A.44) y (A.45) resultaron de la única suposición de despreciar las correlaciones iniciales. El caso $n=2$ será el caso correspondiente a colisiones binarias, los órdenes superiores estarán relacionados a colisiones entre tres y más partículas. La ecuación cinética o de transporte para $W_{+}(x, k)$ se obtiene eliminando $W_{+\left({ }_{i n}\right)}(x, k)$ de ambas (A.44) y (A.45) y considerando las primeras contribuciones no despreciables del desarrollo. Entonces considerando el primer término de (A.44) y sustituyéndolo en (A.45) para $n=2$ obtenemos

$$
k^{\mu} \partial_{\mu} W_{+}(x, k)=\frac{1}{2} \int d^{4} x_{2} d^{4} p_{2} \Delta_{2}\left(x_{2}, p_{2} ; k\right) \prod_{j=1}^{2} W_{+}\left(x+x_{j}, p_{j}\right),
$$


esta es una ecuación es para $W_{+}(x, k)$. Dado que se supuso que la distribución de Wigner varia lento macroscópicamente se puede hacer un desarrollo de Taylor

$$
W_{+}\left(x+x_{j}, p_{j}\right)=W_{+}\left(x, p_{j}\right)+x_{j}^{\mu} \partial_{\mu} W_{+}\left(x, p_{j}\right)+\ldots, \quad j=1,2,
$$

el caso más sencillo, correspondiente a omitir las no uniformidades y desplazamientos de masa, es considerar únicamente el primer término de (A.49) con lo cual la ecuación de transporte resulta

$$
k^{\mu} \partial_{\mu} W_{+}(x, k)=\frac{(2 \pi \hbar)^{6}}{2} \int d^{4} p_{2}\left\{p_{2}\left|\Delta_{+}(k)\right| p_{2}\right\} \prod_{j=1}^{2} W_{+}\left(x, p_{j}\right),
$$

donde se integró en las coordenadas espacio temporales y únicamente quedan los elementos de matriz de $\Delta_{+}(k)$ en el integrando, en la cual podemos hacer la integración sobre la variable auxiliar y dando como resultado

$$
\begin{aligned}
\left\{p_{2}\left|\Delta_{+}(k)\right| p_{2}\right\} & ={ }_{i n}\left\langle p_{2}\left|\Delta_{+}(k)\right| p_{2}\right\rangle_{\text {in }} \\
& =i \int \frac{d^{3} p^{\prime}}{p^{\prime 0}} H\left(k^{0}\right) H\left(k^{2}\right) \delta^{(4)}\left(k+p^{\prime}-p_{1}-p_{2}\right)_{\text {in }}\left\langle p_{1}, p_{2}\left|\phi^{\dagger}(0)\right| p^{\prime}\right\rangle_{\text {out out }}\left\langle p^{\prime}|\varrho(0)| p_{1}, p_{2}\right\rangle_{\text {in }}(A)
\end{aligned}
$$

con ayuda de la ecuación de Yang-Feldman se calcula el braket de $\phi^{\dagger}$ en términos de $\varrho^{\dagger}$ y lo correspondiente para $\phi$, y se puede mostrar que

$$
(2 \pi \hbar)^{6}{ }_{i n}\left\langle p_{2}\left|\Delta_{+}(k)\right| p_{2}\right\rangle_{i n}=\int \frac{d^{3} p}{p^{0}} \delta^{(4)}(k-p) w_{+}\left(p_{1}, p_{2} \mid p\right),
$$

donde la función $w_{+}$se define de la siguiente manera

$$
\begin{aligned}
w_{+}\left(p_{1}, p_{2} \mid p\right)= & \frac{(2 \pi)^{7} \hbar^{8}}{2} \int \frac{d^{3} p^{\prime}}{p^{\prime 0}} \delta^{(4)}\left(p+p^{\prime}-p_{1}-p_{2}\right)\left|{ }_{\text {out }}\left\langle p^{\prime}|\varrho(0)| p_{1}, p_{2}\right\rangle_{\text {in }}\right|^{2} \\
& -\frac{(2 \pi \hbar)^{5} p^{0}}{\sqrt{\pi}}\left[\delta^{(3)}\left(\mathbf{p}-\mathbf{p}_{1}\right) \mathfrak{I m}\left(_{\text {out }}\left\langle p_{2}|\varrho(0)| p_{1}, p_{2}\right\rangle_{\text {in }}\right)+(1 \leftrightarrow 2)\right]
\end{aligned}
$$

Los tres términos del lado derecho de (A.53) pueden interpretarse en términos de amplitudes de dispersión identificando

$$
\left\langle p, p^{\prime}|\mathcal{T}| p_{1}, p_{2}\right\rangle={\frac{(2 \pi \hbar)^{4}}{2 \sqrt{\pi}}}_{\text {out }}\left\langle p^{\prime}|\varrho(0)| p_{1}, p_{2}\right\rangle_{\text {in }}
$$

donde $\mathcal{T}$ es la matriz de transición del proceso de interacción binaria. De tal forma que la función $w_{+}$ puede escribirse como

$$
\begin{aligned}
& w_{+}\left(p_{1}, p_{2} \mid p\right)=\int \frac{d^{3} p^{\prime}}{p^{\prime 0}} \delta^{(4)}\left(p+p^{\prime}-p_{1}-p_{2}\right)\left|\left\langle p, p^{\prime}|\mathcal{T}| p_{1}, p_{2}\right\rangle\right|^{2} \\
& -4 \pi \hbar p^{0}\left[\delta^{(3)}\left(\mathbf{p}-\mathbf{p}_{1}\right) \mathfrak{I m}\left(\left\langle p_{1}, p_{2}|\mathcal{T}| p_{1}, p_{2}\right\rangle\right)+(1 \leftrightarrow 2)\right],
\end{aligned}
$$

de esta forma al tener el Lagrangiano de interacción puede calcularse (A.55) por teoría de perturbaciones. 
Finalmente sustituiremos estos resultados en (A.50), junto con la siguiente representación de la función de Wigner compatible con que $k^{\mu}$ también satisface la restricción de la capa de masa (A.20). Con esto la ecuación de transporte para $f(x, p)$ será la siguiente

$$
p^{\mu} \partial_{\mu} f(x, p)=\frac{1}{2} \int \frac{d^{3} p^{\prime}}{p^{\prime} 0} \frac{d^{3} p_{1}^{\prime}}{p_{1}^{\prime 0}} w_{+}\left(p^{\prime}, p_{1}^{\prime} \mid p\right) f\left(x, p^{\prime}\right) f\left(x, p_{1}^{\prime}\right) .
$$

Un paso final es utilizar un resultado de teoría cuántica de la dispersión conocido como el teorema óptico y es el siguiente

$$
4 \pi \hbar \mathfrak{I m}\left\langle p, p_{1}|\mathcal{T}| p, p_{1}\right\rangle=\frac{1}{2} \int \frac{d^{3} p^{\prime}}{p^{\prime 0}} \frac{d^{3} p_{1}^{\prime}}{p_{1}^{\prime 0}} \delta^{(4)}\left(p^{\prime}+p_{1}^{\prime}-p-p_{1}\right)\left|\left\langle p^{\prime}, p_{1}^{\prime}|\mathcal{T}| p, p_{1}\right\rangle\right|^{2} .
$$

Con este resultado la ecuación de transporte (A.56) puede escribirse como

$$
p^{\mu} \partial_{\mu} f=\int \frac{d^{3} p_{1}}{p_{1}^{0}} \frac{d^{3} p^{\prime}}{p^{\prime} 0} \frac{d^{3} p_{1}^{\prime}}{p_{1}^{\prime 0}}\left[f^{\prime} f_{1}^{\prime} W\left(p^{\prime}, p_{1}^{\prime} \mid p, p_{1}\right)-f f_{1} W\left(p, p_{1} \mid p^{\prime}, p_{1}^{\prime}\right)\right],
$$

donde se introdujo la tasa de transición en términos de la matriz $\mathcal{T}$

$$
W\left(p^{\prime}, p_{1}^{\prime} \mid p, p_{1}\right)=\frac{1}{2} \delta^{(4)}\left(p+p_{1}-p^{\prime}-p_{1}^{\prime}\right)\left|\left\langle p^{\prime}, p_{1}^{\prime}|\mathcal{T}| p, p_{1}\right\rangle\right|^{2}
$$

La ecuación (A.58) es precisamente la expresión (2.12); sin embargo, está última derivación nos permite saber exactamente el régimen de validez de la ecuación de Boltzmann relativista. Además, con este método podemos saber el origen microscópico de la función de distribución y su carácter escalar, así como la tasa de transición en términos de propiedades mecánico cuánticas. 
A.3. Ecuación de Transporte Relativista 


\section{Partículas Relativistas Interactuantes}

\section{B.1. Teorema de No - Interacción de Partículas Relativistas}

El principio de la relatividad establece que todos los observadores en marcos de referencia inerciales obedecen las mismas leyes de la física, esto implica que estas son invariantes ante transformaciones de Lorentz o de Poincaré que incluyen traslaciones espacio-temporales, o bien, en el límite de bajas velocidades respetan las transformaciones de Galileo. De esta forma una teoría será invariante relativista si tiene diez generadores que proveen una representación del álgebra de Poincaré. En el caso de la mecánica clásica (no cuántica) existe otra propiedad conocida como imposición de la línea de mundo. Esta consiste en pedir que la curva de la línea de mundo de una partícula $(q(t), t)$ transforme como las componentes del cuatro vector de posición bajo transformaciones de Lorentz. Esta condición puede expresarse en términos de los generadores del álgebra y la posición de la partícula

$$
\left\{K_{i}, q_{j}\right\}=q_{i}\left[\mathcal{H}, q_{j}\right]
$$

donde $K_{i}$ son los generadores de las transformaciones de Lorentz (boost) en la dirección $i$, y $\mathcal{H}$ es el Hamiltoniano y $\{\cdot, \cdot\}$ son los paréntesis de Poisson que dan la dinámica. En particular para partículas libres relativistas el generador de boosts es $K_{i}=q_{i} \mathcal{H}$, por lo que (B.1) es satisfecha trivialmente.

En un trabajo clásico, Currie, Jordan y Sudarshan [59] analizaron las consecuencias que estas dos condiciones juntas tienen sobre la teoría. Comenzando por escribir a los diez generadores del álgebra de Poincaré $K_{i}, P_{i}, J_{i}, \mathcal{H}$, como funciones de las variables canónicas $q_{i}, p_{i}$ a un tiempo fijo. Concluyen que teorías que contienen ambas condiciones no pueden contener ninguna interacción. A este resultado se le conoce como el teorema de no-interacción de partículas relativistas.

Para enunciar y dar una prueba de este teorema debemos preguntarnos, dado un conjunto de partículas que tienen la simetría de Poincaré, sí sus líneas de mundo transforman apropiadamente bajo cambios de marco de referencia incluso si las partículas tienen una influencia mutua a través de algún tipo de 
interacción. Ambas condiciones sobre la simetría de Poincaré y la invariancia de la línea de mundo son distintas y su efecto da ciertas limitantes a la dinámica final permisible.

Primeramente es necesario tener una formulación geométrica de la dinámica de las partículas. Supongamos que el espacio de configuraciones $Q$ es una variedad diferenciable en la que introducimos localmente ciertas coordenadas $q_{i}$. Para describir al sistema necesitamos ecuaciones diferenciales de al menos primer orden, geométricamente estos son campos vectoriales. Estos campos están definidos en el espacio tangente $T Q$ que se identifica con el espacio fase de velocidades, con coordenadas $\left(q^{i}, \dot{q}^{i}\right)$. La mecánica Lagrangiana sucede en $T Q$. La función Lagrangiana se define como un mapeo tal que $\mathcal{L}: T Q \rightarrow \mathbb{R}$. La mecánica Hamiltoniana toma lugar en el espacio cotangente $T Q^{*}$, que es el espacio fase de momentos con coordenadas $\left(q^{i}, p_{i}\right)$.

Aparentemente la descripción en el espacio cotangente es mejor que en el espacio tangente debido a que las ecuaciones diferenciales de primer orden pueden relacionarse directamente a campos vectoriales y porque el espacio cotangente tiene una estructura simpléctica. Es decir, el espacio cotangente es importante desde el punto de vista geométrico dado que contiene una uno-forma natural $\theta \equiv p_{i} d q^{i}$, tal que la forma simpléctica asociada será $\omega=d \theta=d q^{i} \wedge d p_{i}$. El Hamiltoniano es una función tal que $\mathcal{H}: T Q \rightarrow \mathbb{R}$, de tal forma que las ecuaciones de movimiento pueden escribirse como el siguiente campo sobre $T Q^{*}$

$$
X_{\mathcal{H}}=\left(\frac{\partial \mathcal{H}}{\partial p_{i}},-\frac{\partial \mathcal{H}}{\partial q^{i}}\right)
$$

Se introduce el producto interior como

$$
i_{X_{\mathcal{H}}} \omega=\frac{\partial \mathcal{H}}{\partial p_{i}} d p_{i}+\frac{\partial \mathcal{H}}{\partial q^{i}} d q^{i}=d \mathcal{H},
$$

o bien en términos de la derivada de Lie a lo largo de algún campo $Y, \omega\left(X_{\mathcal{H}}, Y\right)=£_{Y} \mathcal{H}$. Además cualquier campo vectorial Hamiltoniano crea un flujo local que deja invariante la forma simpléctica $d\left(i_{X_{\mathcal{H}}} \omega\right)=$ $£_{X_{\mathcal{H}}} \omega=0$.

Aunque no hay un isomorfismo natural entre $T Q$ y $T Q^{*}$, uno puede definir una uno-forma y por tanto

una forma simpléctica en $T Q$ en términos del Lagrangiano reemplazando $p_{i} \rightarrow \frac{\partial \mathcal{L}}{\partial \dot{q}^{i}}$, de tal modo que la uno-forma correspondiente en $T Q$ será

$$
\theta_{\mathcal{L}} \equiv \frac{\partial \mathcal{L}}{\partial \dot{q}^{i}} d q^{i}
$$

mientras que la forma simpléctica tomará la forma

$$
\omega_{\mathcal{L}} \equiv d \theta_{\mathcal{L}}=\frac{\partial^{2} \mathcal{L}}{\partial \dot{q}^{i} \partial \dot{q}^{j}} d q^{i} \wedge d \dot{q}^{j}+\frac{1}{2}\left(\frac{\partial^{2} \mathcal{L}}{\partial \dot{q}^{i} \partial q^{j}}-\frac{\partial^{2} \mathcal{L}}{\partial \dot{q}^{j} \partial q^{i}}\right) d q^{j} \wedge d q^{i}
$$

La 2-forma $\omega_{\mathcal{L}}$ es invertible y por tanto el Lagrangiano es no degenerado sólo si

$$
\operatorname{det}\left(\frac{\partial^{2} \mathcal{L}}{\partial \dot{q}^{i} \partial \dot{q}^{j}}\right) \neq 0
$$


De (B.5) se desprenden las siguientes relaciones cuando consideramos partículas distintas $a$ y $b$

$$
\begin{aligned}
\omega_{\mathcal{L}}\left(\frac{\partial}{\partial \dot{q}_{(a)}^{i}}, \frac{\partial}{\partial \dot{q}_{(b)}^{j}}\right) & =0 \\
\omega_{\mathcal{L}}\left(\frac{\partial}{\partial \dot{q}_{(a)}^{i}}, \frac{\partial}{\partial q_{(b)}^{j}}\right) & =\frac{\partial^{2} \mathcal{L}}{\partial \dot{q}_{(a)}^{i} \partial \dot{q}_{(b)}^{j}}, \\
\omega_{\mathcal{L}}\left(\frac{\partial}{\partial q_{(a)}^{i}}, \frac{\partial}{\partial q_{(b)}^{j}}\right) & =\frac{1}{2}\left(\frac{\partial^{2} \mathcal{L}}{\partial \dot{q}_{(a)}^{i} \partial q_{(b)}^{j}}-\frac{\partial^{2} \mathcal{L}}{\partial \dot{q}_{(b)}^{i} \partial q_{(a)}^{j}}\right) .
\end{aligned}
$$

Para el problema específico de muchas partículas las etiquetas $a, b, c, \ldots$ corren de $1, \ldots, N$, donde $N$ es el número total de partículas, así $q_{(a)}^{i}$ son las coordenadas de configuración de la partícula $a$.

Con ayuda de la forma (B.5) podemos definir un mapeo $E: T Q \rightarrow \mathbb{R}$ que llamamos la energía y que localmente podemos escribir como

$$
E=\frac{\partial \mathcal{L}}{\partial \dot{q}^{i}} \dot{q}^{i}-\mathcal{L}
$$

Podemos definir un campo vectorial $X_{E}$ que esté dado por la suma $X_{E}=\sum_{a} X_{E}^{(a)}$, donde cada $X_{E}^{(a)}$ en $T Q$ de la partícula $a$, obedezca las ecuaciones de Euler-Lagrange

$$
X_{E}^{(a)}=\dot{q}_{(a)}^{i} \frac{\partial}{\partial q_{(a)}^{i}}+\ddot{q}_{(a)}^{i} \frac{\partial}{\partial \dot{q}_{(a)}^{i}} .
$$

Las ecuaciones de movimiento se vuelven condiciones algebraicas sobre el campo $X_{E}$ :

$$
i_{X_{E}} \omega_{\mathcal{L}}=d E
$$

esta última ecuación implica que $£_{X_{E}} \omega_{\mathcal{L}}=0$.

Además supondremos que el álgebra de Lie del grupo de Poincaré está representada por campos vectoriales en $T Q$ que obedecen ecuaciones similares a la anterior. Los campos $X_{P_{i}}, X_{J_{i}}, X_{K_{i}}$ que generan traslaciones espaciales, rotaciones y transformaciones de Lorentz deben satisfacer

$$
£_{X_{P_{i}}} \omega_{\mathcal{L}}=£_{X_{J_{i}}} \omega_{\mathcal{L}}=£_{X_{K_{i}}} \omega_{\mathcal{L}}=0,
$$

los diez campos anteriores incluyendo $X_{E}$ obedecen las relaciones de conmutación correspondientes al algebra de Lie del grupo de Poincaré. Para el análisis del teorema de no-interacción únicamente utilizaremos la siguiente relación

$$
\left[X_{K_{i}}, X_{E}\right]=X_{P_{i}}
$$

La forma de los campos $X_{P_{i}}$ y $X_{J_{i}}$ es inmediata

$$
\begin{aligned}
& X_{P_{i}}=-\sum_{a} \frac{\partial}{\partial q_{(a)}^{i}}, \\
& X_{J_{i}}=-\sum_{a} \sum_{j k} \varepsilon_{i j k}\left[q_{(a)}^{j} \frac{\partial}{\partial q_{(a)}^{k}}+\dot{q}_{(a)}^{j} \frac{\partial}{\partial \dot{q}_{(a)}^{k}}\right],
\end{aligned}
$$


la estructura del campo $X_{K_{i}}$ es un poco más complicada, las expresiones anteriores para las traslaciones y rotaciones tienen forma de partícula libre, de tal forma que el campo asociado con las traslaciones temporales $X_{E}$ debe cambiar para tomar en cuenta interacciones entre las partículas. Estas consideraciones son importantes en la forma del campo asociado con los boosts de Lorentz ya que la relación de conmutación que este satisface es la siguiente

$$
\left[X_{K_{i}}, X_{P_{j}}\right]=\delta_{i j} X_{E}
$$

de esta forma si $X_{E}$ incluye interacciones y $X_{P_{j}}$ no, entonces $X_{K_{i}}$ debería incluirlas, sin embargo, esto significaría que las líneas de mundo de las partículas no transformarían como las de partículas libres y por tanto no respetarían las transformaciones de Lorentz. Las transformaciones de Lorentz son transformaciones canónicas que mapean ciertas condiciones físicas de un marco de referencia a otro, es en este contexto en que se establece la condición de línea de mundo de las partículas. En el espacio tangente esta condición se puede escribir como

$$
£_{X_{K_{i}}} q_{(a)}^{j}=q_{(a)}^{i} \dot{q}_{(a)}^{j},
$$

que significa tal y cual cosa.... Para determinar la forma del campo $X_{K_{i}}$ se aplica el operador $£_{X_{E}}$ a la condición (B.18) resultando

$$
£_{X_{K_{i}}} \dot{q}_{(a)}^{j}=-\delta_{i j}+\dot{q}_{(a)}^{i} \dot{q}_{(a)}^{j}+q_{(a)}^{i} \ddot{q}_{(a)}^{j},
$$

por lo que el campo asociado a los boosts de Lorentz es

$$
X_{K_{i}}=\sum_{a} q_{(a)}^{i} X_{E}^{(a)}+\sum_{a} \sum_{j}\left(\dot{q}_{(a)}^{i} \dot{q}_{(a)}^{j}-\delta_{i j}\right) \frac{\partial}{\partial \dot{q}_{(a)}^{j}} .
$$

Los generadores de transformaciones de Lorentz están determinados por las ecuaciones de movimiento en la misma medida que la aceleración.

Para obtener el teorema de no-interacción hay que seguir los siguientes pasos:

1. Se aplica la derivada de Lie $£_{X_{K_{k}}}$ a la identidad (B.7) utilizando (B.13) y la forma explícita del generador de boosts de Lorentz (B.20), dando como resultado que

$$
\left(q_{(a)}^{k}-q_{(b)}^{k}\right) \frac{\partial^{2} \mathcal{L}}{\partial \dot{q}_{(a)}^{i} \partial \dot{q}_{(b)}^{j}}=0
$$

esto quiere decir que para partículas distintas $a \neq b$, La segunda derivada cruzada del Lagrangiano debe desaparecer, esto implica una descomposición de la forma

$$
\mathcal{L}=\sum_{a} \mathcal{L}^{(a)}\left(q, \dot{q}_{(a)}\right)
$$

2. Utilizando (B.21) en la propiedad (B.8) resulta que

$$
\omega_{\mathcal{L}}\left(\frac{\partial}{\partial \dot{q}_{(a)}^{i}}, \frac{\partial}{\partial q_{(b)}^{j}}\right)=0,
$$


siempre que $a \neq b$. Aplicando de nuevo la derivada de Lie $£_{X_{K_{k}}}$ al resultado previo se obtiene la siguiente relación

$$
\left(q_{(a)}^{k}-q_{(b)}^{k}\right) \omega_{\mathcal{L}}\left(\frac{\partial}{\partial q_{(a)}^{i}}, \frac{\partial}{\partial q_{(b)}^{j}}\right)=0
$$

para $a \neq b$. Utilizando la última condición (B.9) y la descomposición del Lagrangiano que se obtuvo antes (B.22) se obtiene que

$$
\frac{\partial^{2} \mathcal{L}^{(a)}}{\partial \dot{q}_{(a)}^{i} \partial q_{(b)}^{j}}=\frac{\partial^{2} \mathcal{L}^{(b)}}{\partial \dot{q}_{(b)}^{i} \partial q_{(a)}^{j}}
$$

para partículas distintas. De la condición (B.25) se sigue que los términos en el Lagrangiano que dependan de $\dot{q}_{(a)}$ de forma no lineal, no pueden estar acoplados con términos que dependan de $q_{(b)}$ de tal forma que el Lagrangiano se vuelve a desacoplar como

$$
\mathcal{L}=\sum_{a} \mathcal{L}_{n l}^{(a)}\left(q_{(a)}, \dot{q}_{(a)}\right)-V(q)
$$

donde el término $\mathcal{L}_{n l}^{(a)}$ contiene las contribuciones no lineales al Lagrangiano. Además esta separación hace que la forma simpléctica también se separe como $\omega_{\mathcal{L}}=\sum_{a} \omega_{\mathcal{L}}^{(a)}$, donde cada elemento de la suma tiene la forma $\omega_{\mathcal{L}}^{(a)}=\sum_{i} d\left(\frac{\partial \mathcal{L}_{n l}^{(a)}}{\partial \dot{q}_{(a)}}\right) \wedge d q_{(a)}$.

3. Finalmente se aplica la derivada de Lie $£_{X_{E}} \mathrm{y} £_{X_{K_{k}}}$ a la ecuación (B.24) dando como resultado

$$
\left(q_{(a)}^{l}-q_{(b)}^{l}\right) \sum_{j} \omega_{\mathcal{L}}^{(a)}\left(\frac{\partial}{\partial q_{(a)}^{i}}, \frac{\partial}{\partial \dot{q}_{(b)}^{j}}\right) \frac{\partial \ddot{q}_{(a)}^{j}}{\partial q_{(b)}^{k}}=0,
$$

que implica

$$
\frac{\partial}{\partial q_{(b)}^{k}} \sum_{j} \omega_{\mathcal{L}}^{(a)}\left(\frac{\partial}{\partial q_{(a)}^{i}}, \frac{\partial}{\partial \dot{q}_{(b)}^{j}}\right) \ddot{q}_{(a)}^{j}=0, \quad a \neq b .
$$

En esta ecuación podría haber dependencia en $q_{(b)}^{j}$ a través de las aceleraciones, sin embargo no nos da ninguna condición sobre el potencial $V(q)$ en (B.26). Sin embargo, introduciendo todos estos resultados en la ecuación dinámica (B.12) podemos escribir en una carta local lo siguiente:

$$
-\frac{\partial \mathcal{L}_{n l}^{(a)}}{\partial q_{(a)}^{i}}\left(q_{(a)}, \dot{q}_{(a)}\right)+\sum_{j}\left[\frac{\partial^{2} \mathcal{L}_{n l}^{(a)}}{\partial \dot{q}_{(a)}^{j} \partial \dot{q}_{(a)}^{i}}\left(q_{(a)}, \dot{q}_{(a)}\right) \ddot{q}_{(a)}^{j}+\frac{\partial^{2} \mathcal{L}_{n l}^{(a)}}{\partial \dot{q}_{(a)}^{i} \partial q_{(a)}^{j}}\left(q_{(a)}, \dot{q}_{(a)}\right) \dot{q}_{(a)}^{j}\right]=-\frac{\partial V(q)}{\partial q_{(a)}^{i}} .
$$

Como todas las derivadas del Lagrangiano son independientes de $q_{(b)}$, para $a \neq b$, el potencial $V(q)$ es necesariamente separable tal que

$$
\mathcal{L}=\sum_{a} \mathcal{L}_{n l}^{(a)}\left(q_{(a)}, \dot{q}_{(a)}\right)-V^{(a)}\left(q_{(a)}\right) .
$$

De esta forma se establece que dadas las condiciones sobre el álgebra de Poincaré y la covariancia de las líneas de mundo se tiene que las partículas que satisfacen tales condiciones siguen trayectorias independientes entre sí, es decir no interactúan. 
Además puede mostrarse que, la invariancia de Poincaré implica que $V^{(a)}$ es cero, mientras que la parte no lineal del Lagrangiano toma la forma [208]

$$
\mathcal{L}_{n l}^{(a)}\left(q_{(a)}, \dot{q}_{(a)}\right)=m_{(a)} \sqrt{1-\sum_{i} \dot{q}_{(a)}^{i} \dot{q}_{(a)}^{i}} .
$$

Podemos resumir las hipótesis del teorema de no-interacción: (a) invariancia relativista, (b) dinámica Hamlitoniana y (c) variables de partícula independiente. Se relajara alguna de las condiciones anteriores podríamos preguntarnos que sucedería. Si se elimina la condición (a) obtendríamos la dinámica clásica la cual claramente permite interacciones. Si la condición (b) se elimina tenemos una teoría tipo Newtoniana que es consistente con interacciones. Esta es la formulación de Feynman y Wheeler de las teorías relativistas de acción a distancia [58]. Uno de los problemas con estas teorías que no se pueden cuantizar canónicamente [61]. En [210] se formula un esquema para las interacciones de partículas relativistas, en dicho modelo no pueden establecerse condiciones iniciales adecuadas, además la conservación de la energía y el momento sólo puede asegurarse incluyendo un campo entre las partículas.

Si no se considera la condición (c) también se tiene una teoría con interacciones que es covariante manifiesta pero está formulada en términos de variables distintas a las de partículas independientes. La elección de estas variables se hace de tal forma que cumpla con las restricciones que impone la realización del algebra de Poinacré. Las variables utilizadas no pueden relacionarse con las variables de partícula independiente en presencia de interacciones [61]. Por ejemplo utilizando coordenadas del centro de masa [209], Sin embargo, definir la posición del centro de masa es ambiguo, existen varias posibilidades y no todas corresponden a vectores de Lorentz [61]. Rohrlich muestra en [61] un sistema Hamiltoniano con constricciones para el cual hay interacción en ciertas variables, y como con las variables de partícula son inadecuadas para el caso de interacciones. La formulación con constricciones permite cierto tipo de interacción pero falla en las descomposiciones de cluster. Existen trabajos donde un Hamiltoniano aproximado para interacciones, resuelve la descomposición de cluster. y la condición de la línea de mundo [211]. En [212] se prueba que es necesario tener un generador más en un formalismo con constricciones para tomar en cuenta interacciones, además el generador extra provee las ecuaciones de movimiento [213], Las trayectorias del sistema de dos partículas relativistas interactuantes están generadas por 2 constricciones Hamiltonianas en lugar de un sólo Hamiltoniano [214]. Todos estos modelos están basados en un formalismo dinámica generalizada de constricciones y aunque parecería que el teorema no se aplica en presencia de constricciones, la versión Lagrangiana que se presentó aquí, muestra que sí es válido al menos para constricciones de primera clase [60].

De esta forma la única manera de concebir interacciones entre partículas es a través de incluir los nuevos grados de libertad de un campo mediador que tenga su propio momento y energía. Este sistema combinado lleva a una realización del álgebra de Poincaré en un marco canónico y que satisface las condiciones de la línea de mundo. De ahí que la forma natural de estudiar partículas relativistas interactuantes es en el marco de una teoría de campo. 


\section{Funciones de Bessel modificadas}

En este apéndice se describen algunas propiedades de las dos soluciones a la ecuación de Bessel modificada (C.1).

Las funciones de Bessel modificadas de segunda especie $I_{ \pm n}(z)$ y $K_{n}(z)$ satisfacen la siguiente ecuación diferencial

$$
z^{2} \frac{\partial^{2} w}{\partial z^{2}}+z \frac{\partial w}{\partial z}-\left(z^{2}+n^{2}\right) w=0
$$

Cada solución es una función regular de $z$ en todo el corte del plano $z$ a lo largo del eje real negativo, y cuando $z \neq 0$ es fija, las soluciones serán funciones enteras del índice $n$. Las soluciones $I_{ \pm n}(z)$ y $K_{n}(z)$ son reales y positivas para $n>-1$ y $z>0$. $I_{n}(z)$ es acotada cuando $z \rightarrow 0$, mientras que $K_{n}(z)$ tiende a cero cuando $|z| \rightarrow \infty$ [68]. El comportamiento de las soluciones puede verse en la figura C.1

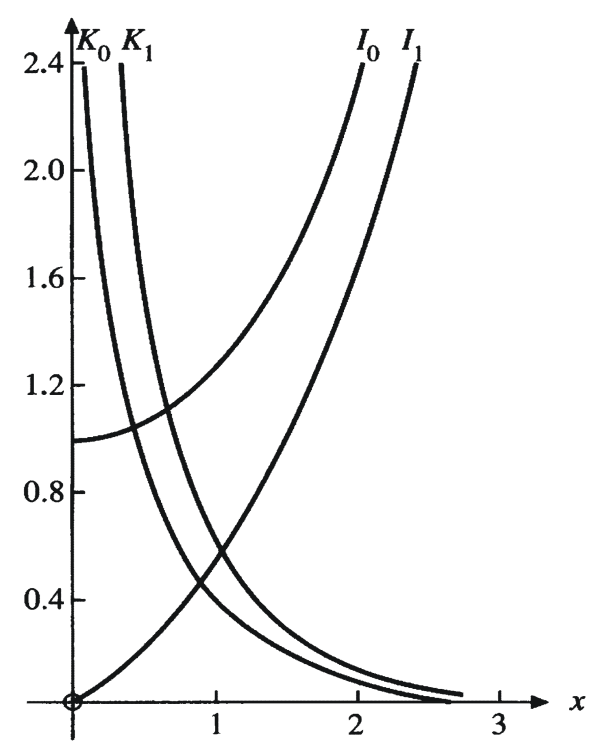

Figura C.1: Funciones de Bessel modificadas de primer y seguda especie [68, 215]. 


\section{C.1. Primera solución $I_{n}(z)$}

La primera solución admite la siguiente representación integral

$$
I_{n}(z)=\frac{1}{\pi} \int_{0}^{\pi} e^{z \cos \vartheta} \cos (n \vartheta) d \vartheta
$$

para $n>-\frac{1}{2}$ otra representación integral es

$$
I_{n}(z)=\left(\frac{z}{2}\right)^{n} \frac{1}{\Gamma\left(\frac{1}{2}\right) \Gamma\left(n+\frac{1}{2}\right)} \int_{-1}^{1} e^{ \pm z x}\left(1-x^{2}\right)^{n-\frac{1}{2}} d x
$$

Se tienen además las siguientes series asociadas con $I_{n}(z)[68,215]$

$$
\begin{aligned}
e^{z \cos \theta} & =I_{0}(z)+2 \sum_{k=1}^{\infty} I_{k}(z) \cos k \theta, \\
\cosh z & =I_{0}(z)+2 \sum_{k=1}^{\infty} I_{2 k}(z), \\
e^{z} & =I_{0}(z)+2 \sum_{k=1}^{\infty} I_{k}(z), \\
I_{n}(z) & =\sum_{k=0}^{\infty} \frac{z^{k}}{k !} J_{n+k}(z) .
\end{aligned}
$$

Para $I_{n}(z)$ se tienen las siguientes relaciones de recurrencia

$$
\begin{gathered}
I_{n-1}(z)-I_{n+1}(z)=\frac{2 n}{z} I_{n}(z), \\
\frac{d}{d z}\left(\frac{I_{n}(z)}{z^{n}}\right)=\frac{I_{n+1}(z)}{z^{n}} \\
\frac{d}{d z}\left(z^{n} I_{n}(z)\right)=z^{n} I_{n-1}(z) .
\end{gathered}
$$

Cuando $n$ está fija y $|z| \gg 1$ es grande puede hacerse un desarrollo asintótico de $I_{n}(z)$, el cual está dado por la siguiente expresión

$$
\begin{aligned}
I_{n}(z) \cong \frac{e^{z}}{\sqrt{2 \pi z}}\left[1-\frac{4 n^{2}-1}{8 z}+\frac{\left(4 n^{2}-1\right)\left(4 n^{2}-9\right)}{2 !(8 z)^{2}}\right. \\
\left.-\frac{\left(4 n^{2}-1\right)\left(4 n^{2}-9\right)\left(4 n^{2}-25\right)}{3 !(8 z)^{3}}+\ldots\right] .
\end{aligned}
$$

Las soluciones $I_{n}(z)$ tienen la siguiente serie ascendente

$$
I_{n}(z)=\left(\frac{z}{2}\right)^{n} \sum_{k=0}^{\infty} \frac{\left(\frac{z^{2}}{4}\right)^{k}}{k ! \Gamma(n+k+1)} .
$$


Cuando $n$ es fija y positiva, y $z \rightarrow 0$ se puede aproximar como

$$
I_{n}(z) \approx\left(\frac{z}{2}\right)^{n} \frac{1}{\Gamma(1+n)}
$$

Cuando el índice $n$ es entero

$$
I_{n}(z)=I_{-n}(z)
$$

\section{C.2. Segunda solución $K_{n}(z)$}

Una de las representaciones integrales de la función $K_{n}$ es la siguiente

$$
K_{n}(z)=\int_{0}^{\infty} e^{-z \cosh \vartheta} \cosh (n \vartheta) d \vartheta .
$$

Otra representación integral de estas funciones es

$$
K_{n}(z)=\left(\frac{z}{2}\right)^{n} \frac{\Gamma\left(\frac{1}{2}\right)}{\Gamma\left(n+\frac{1}{2}\right)} \int_{1}^{\infty} e^{-z x}\left(x^{2}-1\right)^{n-\frac{1}{2}} d x .
$$

Para la función $K_{n}(z)$ se tienen las siguientes relaciones de recurrencia

$$
\begin{gathered}
K_{n-1}(z)-K_{n+1}(z)=-\frac{2 n}{z} K_{n}(z), \\
\frac{d}{d z}\left(\frac{K_{n}(z)}{z^{n}}\right)=-\frac{K_{n+1}(z)}{z^{n}}, \\
\frac{d}{d z}\left(z^{n} K_{n}(z)\right)=-z^{n} K_{n-1}(z) .
\end{gathered}
$$

La solución $K_{n}(z)$ satisface el siguiente teorema de multiplicación de las funciones de Bessel [68, 82]

$$
K_{n}(\lambda z)=\lambda^{n} \sum_{k=0}^{\infty} \frac{(-1)^{k}\left(\lambda^{2}-1\right)^{k}\left(\frac{1}{2} z\right)^{k}}{k !} K_{n+l}(z),
$$

para $\left|\lambda^{2}-1\right|<1$.

El desarrollo asintótico de $K_{n}(z)$ para valores grandes del parámetro, es decir, para $z \gg 1$, está dada por la siguiente expresión

$$
\begin{aligned}
K_{n}(z)= & \sqrt{\frac{\pi}{2 z}} \frac{1}{e^{z}}\left[1+\frac{4 n^{2}-1}{8 z}+\frac{\left(4 n^{2}-1\right)\left(4 n^{2}-9\right)}{2 !(8 z)^{2}}+\right. \\
& \left.+\frac{\left(4 n^{2}-1\right)\left(4 n^{2}-9\right)\left(4 n^{2}-25\right)}{3 !(8 z)^{3}}+\ldots\right] .
\end{aligned}
$$

Para valores pequeños $z \ll 1$, se tiene la siguiente aproximación para $K_{n}(z)$

$$
\begin{aligned}
K_{0}(z) & \approx-\ln z \\
K_{n}(z) & \approx \frac{1}{2}\left(\frac{2}{z}\right)^{n} \Gamma(z), \quad(n>0) .
\end{aligned}
$$


La correspondiente serie al rededor de valores pequeños de $z$ es

$$
\begin{gathered}
K_{n}(z)=(-1)^{n+1} \sum_{k=0}^{\infty} \frac{\left(\frac{z}{2}\right)^{n+2 k}}{k !(n+k) !}\left[\ln \frac{z}{2}-\frac{1}{2} \psi(k+1)-\frac{1}{2} \psi(n+k+1)\right]+ \\
+\frac{1}{2} \sum_{k=0}^{n-1}(-1)^{k} \frac{(n-k-1) !}{k !\left(\frac{z}{2}\right)^{n-2 k}}
\end{gathered}
$$

donde $\psi(n)$ está definida como

$$
\psi(n+1)=-\gamma+\sum_{k=1}^{n} \frac{1}{k}, \quad \psi(1)=-\gamma
$$

con $\gamma=0.577215664$ es la constante de Euler.

Estas funciones son iguales ante el cambio $n \rightarrow-n$

$$
K_{n}(z)=K_{-n}(z)
$$

para el valor particular $n=1 / 2$ y el cambio $z \rightarrow 2 z$ se tiene que

$$
K_{\frac{1}{2}}(2 z)=\sqrt{\frac{\pi}{4 z}} e^{-2 z}
$$




\section{Integrales de colisión para las aproximaciones de Fokker-Planck}

\section{D}

\section{D.1. Integrales para el movimiento Browniano relativista}

En este apéndice proporcionamos los detalles del cálculo del término de colisión para el caso del movimiento Browniano. Se utilizan las expresiones (4.19) para aproximar el término de colisión, así se sustituye (4.19) en la integral $\mathcal{I}$ de la ecuación $(4.5)$ y, al notar que el ángulo entre los vectores espaciales $\mathrm{g}^{i} \mathrm{y} \mathrm{g}^{i^{\prime}}$ es el ángulo de dispersión $\chi$, se tiene que

$$
\mathcal{I}=\int\left(-\frac{p_{G}^{0}}{c} \Delta \mathrm{g}^{i} \frac{\partial h}{\partial p^{i}}+\frac{\left(p_{G}^{0}\right)^{2}}{2 c^{2}} \Delta \mathrm{g}^{i} \Delta \mathrm{g}^{j} \frac{\partial^{2} h}{\partial p^{i} \partial p^{j}}\right) f_{G}^{(0)} g_{\varnothing} \sigma d \Omega d^{3} p_{G},
$$

sustituyendo la distribución de equilibrio de las partículas del gas,

$$
\mathcal{I}=\mathcal{A} \int\left(-\frac{p_{G}^{0}}{c} \Delta \mathrm{g}^{i} \frac{\partial h}{\partial p^{i}}+\frac{\left(p_{G}^{0}\right)^{2}}{2 c^{2}} \Delta \mathrm{g}^{i} \Delta \mathrm{g}^{j} \frac{\partial^{2} h}{\partial p^{i} \partial p^{j}}\right) e^{-\frac{u_{\alpha} p_{G}^{\alpha}}{k T}}\left(g_{\varnothing} \sigma\right) d \Omega d^{3} p_{G}
$$

donde

$$
\mathcal{A}=\frac{n_{G}}{4 \pi m_{G}^{2} c k T K_{2}(\zeta)} .
$$

Se escribe el elemento de ángulo sólido $d \Omega=\operatorname{sen} \chi d \chi d \epsilon$, donde $\chi \mathrm{y} \epsilon$ son los ángulos polares de $\mathrm{g}^{i^{\prime}}$ respecto a $g^{i}$. Así se puede integrar con respecto a $\epsilon$

$$
\begin{gathered}
\int_{0}^{2 \pi} \Delta \mathrm{g}^{i} d \epsilon=-2 \pi(1-\cos \chi) \mathrm{g}^{i} \\
\int_{0}^{2 \pi} \Delta \mathrm{g}^{i} \Delta \mathrm{g}^{j} d \epsilon=2 \pi\left\{\left[(1-\cos \chi)^{2}-\frac{1}{2} \operatorname{sen}^{2} \chi\right] \mathrm{g}^{i} \mathrm{~g}^{j}-\frac{|\mathbf{g}|^{2}}{2} \eta^{i j} \operatorname{sen}^{2} \chi\right\},
\end{gathered}
$$

sustituyendo las anteriores relaciones en la integral (D.2)

$$
\mathcal{I}=2 \pi \mathcal{A} \int e^{-\frac{u_{\alpha} p_{G}^{\alpha}}{k T}}\left(g_{\varnothing} \sigma\right) \operatorname{sen} \chi d \chi d^{3} p_{G}\left[\frac{p_{G}^{0}}{c} \frac{\partial h}{\partial p^{i}} g^{i}(1-\cos \chi)\right.
$$




$$
\left.+\frac{\left(p_{G}^{0}\right)^{2}}{4 c^{2}} \frac{\partial^{2} h}{\partial p^{i} \partial p^{j}}\left\{\left(1-4 \cos \chi+3 \cos ^{2} \chi\right) \mathrm{g}^{i} \mathrm{~g}^{j}-|\mathbf{g}|^{2} \eta^{i j} \operatorname{sen}^{2} \chi\right\}\right] .
$$

Si se escribe el elemento de volumen del gas $d p_{G}$ en coordenadas esféricas $d^{3} p_{G}=\operatorname{sen} \phi d \phi d \vartheta\left|\mathbf{p}_{G}\right|^{2} d\left|\mathbf{p}_{G}\right|$ y se hace la integración en el ángulo acimutal $\vartheta$ de $\mathrm{g}^{i} \mathrm{y} \mathrm{g}^{i} \mathrm{~g}^{j}$, de la misma manera que se hizo en las integrales (D.4) y (D.5), el resultado es

$$
\begin{gathered}
c \int_{0}^{2 \pi}\left(\frac{p_{G}^{i}}{p_{G}^{0}}-\frac{p^{i}}{p^{0}}\right) d \vartheta=2 \pi c\left(\frac{\left|\mathbf{p}_{G}\right|}{|\mathbf{p}|} \frac{p^{0}}{p_{G}^{0}} \cos \phi-1\right) \frac{p^{i}}{p^{0}}, \\
c^{2} \int_{0}^{2 \pi}\left(\frac{p_{G}^{i}}{p_{G}^{0}}-\frac{p^{i}}{p^{0}}\right)\left(\frac{p_{G}^{j}}{p_{G}^{0}}-\frac{p^{j}}{p^{0}}\right) d \vartheta=2 \pi c^{2}\left\{\left[\left(\frac{\left|\mathbf{p}_{G}\right|}{|\mathbf{p}|} \frac{p^{0}}{p_{G}^{0}} \cos \phi-1\right)^{2}\right.\right. \\
\left.\left.-\frac{1}{2} \frac{\left|\mathbf{p}_{G}\right|^{2}}{|\mathbf{p}|^{2}} \frac{\left(p^{0}\right)^{2}}{\left(p_{G}^{0}\right)^{2}} \operatorname{sen}^{2} \phi\right] \frac{p^{i} p^{j}}{\left(p^{0}\right)^{2}}-\frac{1}{2} \frac{\left|\mathbf{p}_{G}\right|^{2}}{\left(p_{G}^{0}\right)^{2}} \eta^{i j} \operatorname{sen}^{2} \phi\right\} .
\end{gathered}
$$

Si se sustituyen estos resultados en la integral $I$ (D.6) se obtiene que

$$
\begin{gathered}
\mathcal{I}=4 \pi^{2} \mathcal{A} \int e^{-\frac{u_{\alpha} p_{G}^{\alpha}}{k T}}\left(g_{\varnothing} \sigma\right)\left|\mathbf{p}_{G}\right|^{2} d\left|\mathbf{p}_{G}\right| \operatorname{sen} \chi d \chi \operatorname{sen} \phi d \phi \times\left\{(1-\cos \chi) p_{G}^{0} \times\right. \\
\times \frac{\partial h}{\partial p^{i}} \frac{p^{i}}{p^{0}}\left(\frac{\left|\mathbf{p}_{G}\right|}{|\mathbf{p}|} \frac{p^{0}}{p_{G}^{0}} \cos \phi-1\right)+\frac{\left(p_{G}^{0}\right)^{2}}{4} \frac{\partial^{2} h}{\partial p^{i} \partial p^{j}}\left[\left(1-4 \cos \chi+3 \cos ^{2} \chi\right) \times\right. \\
\times\left\{\frac{p^{i} p^{j}}{\left(p^{0}\right)^{2}}\left(\frac{\left|\mathbf{p}_{G}\right|^{2}}{|\mathbf{p}|^{2}} \frac{\left(p^{0}\right)^{2}}{\left(p_{G}^{0}\right)^{2}} \cos ^{2} \phi-2 \frac{\left|\mathbf{p}_{G}\right|}{|\mathbf{p}|} \frac{p^{0}}{p_{G}^{0}} \cos \phi+1-\frac{1}{2} \frac{\left|\mathbf{p}_{G}\right|^{2}}{|\mathbf{p}|^{2}} \frac{\left(p^{0}\right)^{2}}{\left(p_{G}^{0}\right)^{2}} \operatorname{sen}^{2} \phi\right)\right. \\
\left.\left.\left.-\frac{1}{2} \eta^{i j} \frac{\left|\mathbf{p}_{G}\right|^{2}}{\left(p_{G}^{0}\right)^{2}} \operatorname{sen}^{2} \phi\right\}-\frac{|\mathbf{g}|^{2}}{c^{2}} \eta^{i j} \operatorname{sen}^{2} \chi\right]\right\} .
\end{gathered}
$$

Para poder continuar con las aproximaciones a la integral $\mathcal{I}$ se analizará el producto $\left(g_{\varnothing} \sigma\right)$. La sección transversal diferencial en este caso será función del ángulo de dispersión y de el flujo invariante, $\sigma=$ $\sigma(F, \chi)$. De modo tal que para $F$ se puede escribir

$$
\begin{aligned}
F & =\sqrt{\left(p_{\alpha} p_{G}^{\alpha}\right)^{2}-m^{2} m_{G}^{2} c^{4}} \\
& =p^{0}\left|\mathbf{p}_{G}\right| \sqrt{1-2 \frac{|\mathbf{p}|}{p^{0}} \frac{p_{G}^{0}}{\left|\mathbf{p}_{G}\right|} \cos \phi+\frac{|\mathbf{p}|^{2}}{\left(p^{0}\right)^{2}} \operatorname{sen}^{2} \phi+\frac{\left(p_{G}^{0}\right)^{2}}{\left(p^{0}\right)^{2}} \frac{|\mathbf{p}|^{2}}{\left|\mathbf{p}_{G}\right|^{2}}} .
\end{aligned}
$$

Al ser la partícula no relativista se sabe que el cociente $|\mathbf{p}| / p^{0}$ es pequeño, además se supone que el gas es cercanamente relativista $p_{G}^{0} \sim\left|\mathbf{p}_{G}\right|$, de modo que se utilizará como parámetro para aproximar $F$. Despreciando el último término en (D.11), se puede aproximar el producto $F \sigma$, el cual se desarrolla en series de Taylor como sigue

$$
F \sigma(F, \chi) \cong p^{0}\left|\mathbf{p}_{G}\right| \sigma\left(\left|\mathbf{p}_{G}\right|, \chi\right)\left(1-\frac{|\mathbf{p}|}{p^{0}} \frac{p_{G}^{0}}{\left|\mathbf{p}_{G}\right|} \cos \phi\left(1+\frac{\left|\mathbf{p}_{G}\right|}{\sigma} \frac{\partial \sigma}{\partial\left|\mathbf{p}_{G}\right|}\right)\right),
$$


que en términos de la velocidad de Møller queda

$$
g_{\varnothing} \sigma(F, \chi) \cong c \frac{\left|\mathbf{p}_{G}\right|}{p_{G}^{0}} \sigma\left(\left|\mathbf{p}_{G}\right|, \chi\right)\left(1-\frac{|\mathbf{p}|}{p^{0}} \frac{p_{G}^{0}}{\left|\mathbf{p}_{G}\right|} \cos \phi\left(1+\frac{\left|\mathbf{p}_{G}\right|}{\sigma} \frac{\partial \sigma}{\partial\left|\mathbf{p}_{G}\right|}\right)\right) .
$$

Se puede hacer la misma aproximación para el cociente de la magnitud de la velocidad relativa $|\mathbf{g}|^{2} / c^{2}$

$$
\frac{|\mathbf{g}|^{2}}{c^{2}}=\frac{\left|\mathbf{p}_{G}\right|^{2}}{\left(p_{G}^{0}\right)^{2}}+\frac{|\mathbf{p}|^{2}}{\left(p^{0}\right)^{2}}-2 \frac{\left|\mathbf{p}_{G}\right|}{p_{G}^{0}} \frac{|\mathbf{p}|}{p^{0}} \cos \phi
$$

considerando únicamente el primer término de esta ecuación. El hecho de que las partículas pesadas sean no relativistas es necesario para comparar los órdenes del cociente $|\mathbf{p}| / p^{0}$ en la ecuación (D.9); se desprecian todos los términos de orden $|\mathbf{p}| / p^{0}$ y mayores, para que la integral quede a primer orden ${ }^{1}$.

Al sustituir (D.13) y (D.14) en la expresión para $I$ (D.9), dejando únicamente términos de primer orden y realizando la integración en ángulo $\phi$, se obtiene entonces

$$
\begin{gathered}
\mathcal{I}=4 \pi^{2} c \mathcal{A} \int e^{-\frac{u_{\alpha} p_{G}^{\alpha}}{k T}} \sigma\left(\left|\mathbf{p}_{G}\right|, \chi\right)\left|\mathbf{p}_{G}\right|^{3} \frac{d\left|\mathbf{p}_{G}\right|}{p_{G}^{0}} \operatorname{sen} \chi d \chi\left\{-2(1-\cos \chi) p_{G}^{0} \frac{\partial h}{\partial p^{i}} \frac{p^{i}}{p^{0}} \times\right. \\
\times\left[1+\frac{1}{3}\left(1+\frac{\left|\mathbf{p}_{G}\right|}{\sigma} \frac{\partial \sigma}{\partial\left|\mathbf{p}_{G}\right|}\right)\right]+\frac{\left(p_{G}^{0}\right)^{2}}{4} \frac{\partial^{2} h}{\partial p^{i} \partial p^{j}}\left[\left(1-4 \cos \chi+3 \cos ^{2} \chi\right) \times\right. \\
\left.\left.\times\left(-\frac{2}{3} \eta^{i j} \frac{\left|\mathbf{p}_{G}\right|^{2}}{\left(p_{G}^{0}\right)^{2}}\right)-2 \operatorname{sen}^{2} \chi \eta^{i j} \frac{\left|\mathbf{p}_{\mathbf{G}}\right|^{2}}{\left(p_{G}^{0}\right)^{2}}\right]\right\},
\end{gathered}
$$

al factorizar $(1-\cos \chi)$ se tiene que

$$
\begin{gathered}
\mathcal{I}=4 \pi^{2} c \mathcal{A} \int e^{-\frac{u_{\alpha} p_{G}^{\alpha}}{k T}} \sigma\left(\left|\mathbf{p}_{G}\right|, \chi\right)\left|\mathbf{p}_{G}\right|^{3} \frac{d\left|\mathbf{p}_{G}\right|}{p_{G}^{0}}(1-\cos \chi) \operatorname{sen} \chi d \chi \\
\left\{-2 p_{G}^{0} \frac{\partial h}{\partial p^{i}} \frac{p^{i}}{p^{0}}\left[1+\frac{1}{3}\left(1+\frac{\left|\mathbf{p}_{G}\right|}{\sigma} \frac{\partial \sigma}{\partial\left|\mathbf{p}_{G}\right|}\right)\right]-\frac{2}{3}\left(p_{G}^{0}\right)^{2} \frac{\partial^{2} h}{\partial p^{i} \partial p^{j}} \eta^{i j} \frac{\left|\mathbf{p}_{G}\right|^{2}}{\left(p_{G}^{0}\right)^{2}}\right\} .
\end{gathered}
$$

Integrando por partes la derivada de la sección transversal $\sigma$, escogiendo un marco co-móvil en el cual $\mathcal{U}_{\alpha} \rightarrow(c, \mathbf{0})$ y haciendo explícita la dependencia de $p_{G}^{0}$ en $p_{G}^{i}$, se llega a la siguiente relación

$$
\int d\left|\mathbf{p}_{G}\right|\left|\mathbf{p}_{G}\right|^{4} e^{-\frac{u_{\alpha} p_{G}^{\alpha}}{k T}} \frac{\partial \sigma}{\partial\left|\mathbf{p}_{G}\right|}=-\int d\left|\mathbf{p}_{G}\right|\left[4\left|\mathbf{p}_{G}\right|^{3}-\frac{c}{k T p_{G}^{0}}\left|\mathbf{p}_{G}\right|^{5}\right] e^{-\frac{u_{\alpha} p_{G}^{\alpha}}{k T}} \sigma .
$$

Sustituyendo la expresión anterior en (D.16) se obtiene

$$
\begin{gathered}
\mathcal{I}=\frac{8}{3} \pi^{2} c \mathcal{A} \int e^{-\frac{u_{\alpha p_{G}^{\alpha}}^{\alpha}}{k T}} \sigma\left(\left|\mathbf{p}_{G}\right|, \chi\right)\left|\mathbf{p}_{G}\right|^{5} \frac{d\left|\mathbf{p}_{G}\right|}{p_{G}^{0}}(1-\cos \chi) \operatorname{sen} \chi d \chi \times \\
\times\left[-\frac{\partial h}{\partial p^{i}} p^{i} \frac{c}{k T p^{0}}-\eta^{i j} \frac{\partial^{2} h}{\partial p^{i} \partial p^{j}}\right] .
\end{gathered}
$$

\footnotetext{
${ }^{1}$ Esta misma aproximación también resulta al tomar como parámetro el cociente de energías $p_{G}^{0} / p^{0} \ll 1$ notando que, con la restricción en las masas $\left|\mathbf{p}_{G}\right| \sim|\mathbf{p}|$.
} 
Hasta este punto se ha trabajado con la integral $\mathcal{I}$, ahora hay que regresar al término de colisión original multiplicando $\mathcal{I}$ por la función de distribución del equilibrio de las partículas pesadas $f^{(0)}$, Cuando las partículas son no relativistas dicha función tiende a la función de distribución de Maxwell-Boltzmann. De este modo el término de colisión $f^{(0)} \mathcal{I}$ se puede escribir como en la ecuación (4.20)

\section{D.2. Integrales para el gas de Lorentz relativista}

En esta sección se consideran las aproximaciones correspondientes al caso del Gas de Lorentz. Comenzamos sustituyendo (4.27) en (4.5) y utilizando (4.6) Luego integramos con respecto al ángulo $\epsilon$ del ángulo sólido $d \Omega=\sin \chi d \chi d \epsilon$. Para tal efecto se utilizan las siguientes expresiones

$$
\begin{gathered}
\int_{0}^{2 \pi} \Delta \mathrm{g}^{i} d \epsilon=-2 \pi(1-\cos \chi) \mathrm{g}^{i} \\
\int_{0}^{2 \pi} \Delta \mathrm{g}^{i} \Delta \mathrm{g}^{j} d \epsilon=2 \pi\left\{\left[(1-\cos \chi)^{2}-\frac{1}{2} \sin ^{2} \chi\right] \mathrm{g}^{i} \mathrm{~g}^{j}-\frac{|\mathbf{g}|^{2}}{2} \eta^{i j} \sin ^{2} \chi\right\},
\end{gathered}
$$

donde $\eta^{i j}=\operatorname{diag}\{-1,-1,-1\}$ es la parte espacial de la métrica de Minkowski. De tal modo que las integrales pueden escribirse como

$$
\begin{gathered}
\mathcal{I}=2 \pi \int f_{G}^{(0)}\left(g_{\varnothing} \sigma\right) \sin \chi d \chi d^{3} p_{G}\left[\frac{p^{0}}{c} \frac{\partial h}{\partial p^{i}} \mathrm{~g}^{i}(1-\cos \chi)\right. \\
\left.+\frac{\left(p^{0}\right)^{2}}{4 c^{2}} \frac{\partial^{2} h}{\partial p^{i} \partial p^{j}}\left\{\left(1-4 \cos \chi+3 \cos ^{2} \chi\right) \mathrm{g}^{i} \mathrm{~g}^{j}-|\mathbf{g}|^{2} \eta^{i j} \sin ^{2} \chi\right\}\right] .
\end{gathered}
$$

Usando coordenadas esféricas $d^{3} p_{G}=\sin \phi d \phi d \theta\left|\mathbf{p}_{G}\right|^{2} d\left|\mathbf{p}_{G}\right|$ e integrando en los ángulo acimutal $\vartheta$ con

$$
\begin{gathered}
c \int_{0}^{2 \pi}\left(\frac{p_{G}^{i}}{p_{G}^{0}}-\frac{p^{i}}{p^{0}}\right) d \vartheta=2 \pi c\left(\frac{\left|\mathbf{p}_{G}\right|}{|\mathbf{p}|} \frac{p^{0}}{p_{G}^{0}} \cos \phi-1\right) \frac{p^{i}}{p^{0}}, \\
c^{2} \int_{0}^{2 \pi}\left(\frac{p_{G}^{i}}{p_{G}^{0}}-\frac{p^{i}}{p^{0}}\right)\left(\frac{p_{G}^{j}}{p_{G}^{0}}-\frac{p^{j}}{p^{0}}\right) d \vartheta=2 \pi c^{2}\left\{\left[\left(\frac{\left|\mathbf{p}_{G}\right|}{|\mathbf{p}|} \frac{p^{0}}{p_{G}^{0}} \cos \phi-1\right)^{2}\right.\right. \\
\left.\left.-\frac{1}{2} \frac{\left|\mathbf{p}_{G}\right|^{2}}{|\mathbf{p}|^{2}} \frac{\left(p^{0}\right)^{2}}{\left(p_{G}^{0}\right)^{2}} \sin ^{2} \phi\right] \frac{p^{i} p^{j}}{\left(p^{0}\right)^{2}}-\frac{1}{2} \frac{\left|\mathbf{p}_{G}\right|^{2}}{\left(p_{G}^{0}\right)^{2}} \eta^{i j} \sin ^{2} \phi\right\},
\end{gathered}
$$

la integral $\mathcal{I}$ se vuelve

$$
\begin{gathered}
\mathcal{I}=4 \pi^{2} \int f_{G}^{(0)}\left(g_{\varnothing} \sigma\right)\left|\mathbf{p}_{G}\right|^{2} d\left|\mathbf{p}_{G}\right| \sin \chi d \chi \sin \phi d \phi\left\{(1-\cos \chi) p^{0} \times\right. \\
\times \frac{\partial h}{\partial p^{i}} \frac{p^{i}}{p^{0}}\left(\frac{\left|\mathbf{p}_{G}\right|}{|\mathbf{p}|} \frac{p^{0}}{p_{G}^{0}} \cos \phi-1\right)+\frac{\left(p^{0}\right)^{2}}{4} \frac{\partial^{2} h}{\partial p^{i} \partial p^{j}}\left[\left(4(1-\cos \chi)-3 \sin ^{2} \chi\right) \times\right. \\
\times\left\{\frac{p^{i} p^{j}}{\left(p^{0}\right)^{2}}\left(\frac{\left|\mathbf{p}_{G}\right|^{2}}{|\mathbf{p}|^{2}} \frac{\left(p^{0}\right)^{2}}{\left(p_{G}^{0}\right)^{2}} \cos ^{2} \phi-2 \frac{\left|\mathbf{p}_{G}\right|}{|\mathbf{p}|} \frac{p^{0}}{p_{G}^{0}} \cos \phi+1-\frac{1}{2} \frac{\left|\mathbf{p}_{G}\right|^{2}}{|\mathbf{p}|^{2}} \frac{\left(p^{0}\right)^{2}}{\left(p_{G}^{0}\right)^{2}} \sin ^{2} \phi\right)\right.
\end{gathered}
$$




$$
\left.\left.\left.-\frac{1}{2} \eta^{i j} \frac{\left|\mathbf{p}_{G}\right|^{2}}{\left(p_{G}^{0}\right)^{2}} \sin ^{2} \phi\right\}-\frac{|\mathbf{g}|^{2}}{c^{2}} \eta^{i j} \sin ^{2} \chi\right]\right\} \text {. }
$$

Para seguir avanzando necesitamos aproximar la velocidad de Møller. Debido a $p^{0} / p_{G}^{0} \ll 1$ y como el gas no es relativista, se introduce una aproximación al flujo invariante $F=p^{0} p_{G}^{0} g_{\varnothing} c^{-1}$

$$
F \cong p_{G}^{0}|\mathbf{p}|\left(1-\frac{p^{0}}{p_{G}^{0}} \frac{\left|\mathbf{p}_{G}\right|}{|\mathbf{p}|} \cos \phi+\frac{1}{2} \frac{\left(p^{0}\right)^{2}}{\left(p_{G}^{0}\right)^{2}} \frac{\left|\mathbf{p}_{G}\right|^{2}}{|\mathbf{p}|^{2}} \cos ^{2} \phi\right) .
$$

Debido a que la sección transversal $\sigma$ es función de $F$, dado por (D.25), podemos aproximarla como $\sigma(F, \chi) \simeq \sigma(|\mathbf{p}|, \chi)$. A este orden no es independiente de $\left|\mathbf{p}_{G}\right|$. Así, para el producto $g_{\varnothing} \sigma$, con el uso de (D.25), obtenemos ${ }^{2}$

$$
g_{\varnothing} \sigma(F, \chi) \cong c \frac{|\mathbf{p}|}{p^{0}} \sigma(|\mathbf{p}|, \chi)\left(1-\frac{p^{0}}{p_{G}^{0}} \frac{\left|\mathbf{p}_{G}\right|}{|\mathbf{p}|} \cos \phi+\frac{1}{2} \frac{\left(p^{0}\right)^{2}}{\left(p_{G}^{0}\right)^{2}} \frac{\left|\mathbf{p}_{G}\right|^{2}}{|\mathbf{p}|^{2}} \cos ^{2} \phi\right) .
$$

Podemos sustituir (D.26) en (D.24) que junto con la expresión para el último término de (D.24) que es

$$
\frac{|\mathbf{g}|^{2}}{c^{2}}=\frac{|\mathbf{p}|^{2}}{p^{0^{2}}}\left(1-2 \frac{\left|\mathbf{p}_{G}\right|}{|\mathbf{p}|} \frac{\left(p^{0}\right)}{\left(p_{G}^{0}\right)} \cos \phi+\frac{\left|\mathbf{p}_{G}\right|^{2}}{|\mathbf{p}|^{2}} \frac{\left(p^{0}\right)^{2}}{\left(p_{G}^{0}\right)^{2}}\right) .
$$

Como las partículas de gas no son relativistas, podemos considerar que la cantidad $\left|\mathbf{p}_{G}\right| / p_{G}^{0}$ es una pequeña corrección y entonces, despreciar todos los términos de orden superior a 2 . Debido a que las derivadas parciales de $h$ no dependen ni de $\phi$, ni de $\left|\mathbf{p}_{G}\right|$, se puede integrar directamente (D.24) en $\phi$ y $\left|\mathbf{p}_{G}\right|$. La sustitución de la función de distribución de Maxwell $f_{G}^{(0)}$ junto con el uso de $p_{G}^{0} \approx m_{G} c$, nos lleva a la siguiente expresión

$$
\begin{aligned}
\frac{n_{G}}{\left(2 \pi k T m_{G}\right)^{\frac{3}{2}}} \int_{0}^{\infty} e^{-\frac{\left|\mathbf{p}_{G}\right|^{2}}{2 k T m_{G}}}\left|\mathbf{p}_{G}\right|^{2} d\left|\mathbf{p}_{G}\right| & =\frac{n_{G}}{4 \pi} \\
\frac{n_{G}}{\left(2 \pi k T m_{G}\right)^{\frac{3}{2}}} \int_{0}^{\infty} e^{-\frac{\left|\mathbf{p}_{G}\right|^{2}}{2 k T m_{G}}}\left|\mathbf{p}_{G}\right|^{4} d\left|\mathbf{p}_{G}\right| & =\frac{3 n_{G}}{4 \pi} m_{G} k T .
\end{aligned}
$$

De esta forma estamos en condiciones de dar una forma explícita de $\mathcal{I}$, la cual está dada en la ecuación. (4.28).

\footnotetext{
${ }^{2}$ En $[134,12]$ para el estudio de la difusión espacial en un gas de Lorentz relativista, Kox utiliza el término principal en (D.25) y (D.26) para aproximar el flujo invariante.
} 
D.2. Integrales para el gas de Lorentz relativista 


\section{Gravedad Cuántica de Lazos}

\section{E.1. El problema de cuantizar la gravedad}

Uno de los problemas más importantes en la física teórica actual es la implementación consistente de las interacciones gravitacionales dentro del marco de la teoría cuántica, las cuales resultan incompatibles desde sus primeros principios. Una característica importante es la existencia de unidades fundamentales de masa, longitud y tiempo que resultan de la combinación única de las constantes $\hbar, G$ y $c$, estas son conocidas como dimensiones de Planck. Se espera que a estas escalas los efectos cuánticos de la gravedad sean relevantes. Estas escalas llevan a un obstáculo muy importante en la teoría que es que es la inexistencia de datos experimentales directos que puedan dar guía a su construcción, debido a que sus valores son extremadamente grandes para la energía, y extremadamente pequeños para la longitud. Así, los estudios en este ámbito se han enfocado en construir aparatos matemáticos que sean consistentes internamente y compatibles con los conceptos físicos usuales. En realidad, hasta ahora las motivaciones para construir una teoría cuántica de la gravedad son puramente teóricas: Los teoremas de singularidad y la evasión de singularidades, evolución de los hoyos negros, radiación de Hawking y etapa final de evaporación, gran unificación de las interacciones fundamentales, regulador y divergencias en la teoría cuántica de campos. Como enseña la relatividad general, la gravitación está codificada en la geometría del espacio-tiempo, de esta forma se espera que la cuantización de la gravedad esté relacionada con la cuantización de la geometría. El modelo matemático utilizado en la gravitación clásica es una variedad diferenciable equipada con una métrica lorentziana, sin embargo, como muestra Isham [216]-[218], existe una jerarquía de estructuras geométricas donde podría en principio aplicarse la cuantización, desde los eventos espacio-temporales, la topología, la relación causal, la métrica, etc. La pregunta principal en gravedad cuántica es ¿qué tanto de esa estructura debe quedar fija y donde podrán haber fluctuaciones cuánticas? En una teoría fundamental ninguna estructura puede ser absoluta, es decir, se quiere una teoría independiente del fondo. Por ejemplo, en la teoría cuántica de un campo escalar $\phi$, el papel principal es de la condición causal $[\hat{\phi}(x), \hat{\phi}(y)]=0$, 
para todos los puntos del espacio-tiempo separados respecto a la métrica fija. Si se cuantiza la métrica, el cono de luz asociado a cualquier evento ya no es fijo y por lo tanto la relación de conmutación anterior no tiene sentido. Es por eso la necesidad de una teoría que no dependa de una estructura de fondo particular. Al utilizar la teoría cuántica estándar en la gravedad, en particular en Cosmología se encuentra un problema en la tradicional interpretación de Copenhague donde se el universo se separa en dos partes, por un lado se aísla el sistema y por otro el observador que realiza mediciones. Existe un conflicto en este caso al no haber alguien externo al universo que actué como observador. En particular se pretende tener una interpretación que no incluya como elemento fundamental la noción de medición por un observador externo, un ejemplo es el enfoque de historias consistentes.

Uno de los aspectos más importantes que debe abordar una teoría de gravedad cuántica es el problema del tiempo. El tiempo aparece como un parámetro de evolución en la ecuación básica de la mecánica cuántica, la ecuación de Schrödinger, que conserva la noción de tiempo absoluto de la física newtoniana. No es una observable ni está representado por un operador, es la parte clásica de la interpretación de Copenhague donde la noción de medición a un tiempo particular es fundamental; un observable es algo cuyo valor se mide a un tiempo determinado. Por otro lado, en la relatividad general el tiempo es una variable dinámica, parte de la estructura espacio-temporal descrita por las ecuaciones de Einstein. Una variedad con métrica puede foliarse de muchas formas de tal modo que el parámetro en cada foliación puede usarse como definición de tiempo y no hay manera de elegir uno en particular, estos son parámetros temporales no físicos que la teoría permite. En el caso de la gravedad cuántica hay diferentes opiniones acerca del papel que del tiempo. Hay enfoques que utilizan una estructura causal de fondo para determinar los conceptos temporales, otros no tienen al tiempo como un parámetro así que definen tiempos internos para determinar la evolución. Una teoría de la gravedad cuántica necesita decir algo sobre los problemas mencionados, así como recuperar en los límites correctos los resultados ya conocidos.

Existen diversos enfoques para abordar este problema. Están las propuestas que pretenden unificar las interacciones fundamentales como la teoría de cuerdas [219]. También están aquellas que únicamente se preocupan en los efectos cuánticos de la gravedad sobre los campos y viceversa como la gravedad semiclásica y la gravedad estocástica [220]-[225]. En esta dirección existen estrategias que se fijan únicamente en efectos generales como la existencia de una longitud mínima o la posible modificación a la simetría de Lorentz para intentar buscar pruebas experimentales donde los efectos cuánticos del espacio-tiempo puedan amplificarse y medirse a través de cantidades físicas ordinarias. A estos esfuerzos se les llama colectivamente como la fenomenología de gravedad cuántica [226, 227]. Los más conocidos son aquellos donde se modifica la relación de dispersión y principio de incertidumbre para buscar efectos debidos a la posible granularidad del espacio a la escala de Planck.

Basándose en el probable carácter discreto del espacio existen modelos como los conjuntos causales que intentan implementar la causalidad entre los puntos del espacio-tiempo [41, 109, 228]. Entre las predicción más interesantes están que las fluctuaciones en el valor de la constante cosmológica son consistentes con 
las observaciones y que se ha podido reproducir en una aproximación de grano grueso la causalidad en el espacio-tiempo de Minkowski.

En este apéndice damos una revisión sencilla de uno de los enfoques canónicos a la gravedad cuántica conocido como gravedad cuántica de lazos. En el enfoque canónico usual se comienza con una foliación 3+1 de la variedad 4-dimensional, utilizando como variables canónicas la métrica del espacio 3-dimensional $q_{a b}$ y su momento conjugado para describir la dinámica de geometrías 3-dimensionales. El resultado es una teoría clásica constreñida que hay que cuantizar.

La diferencia principal de la gravedad de lazos es que en lugar de utilizar a la métrica como variable de configuración se utilizan nuevas variables que son combinaciones complejas de las variables canónicas estándar, una de las variables es una conexión de espín lo que sugiere el uso de un análogo gravitacional de los lazos de Wilson en la teoría de Yang- Mills. Estas ideas llevan a resultados muy interesantes como que los operadores de área y volumen tienen espectro discreto.

\section{E.2. Gravedad Cuántica de Lazos}

\section{E.2.1. Preliminares}

La gravedad cuántica de lazos es un enfoque independiente del espacio-tiempo de fondo en la construcción de una teoría cuántica de campos para la gravedad ${ }^{1}$. El punto de partida es la formulación Hamiltoniana de la relatividad general, o formulación $A D M[75,231]$. En esta, se hace una foliación del espacio-tiempo en hipersuperficies espaciales. Se estudia la evolución de la geometría espacial en cada una de esas superficies. En el tratamiento usual la métrica y su momento conjugado son las variables del espacio fase que se estudia. Mediante una transformación canónica adecuada se pueden obtener nuevas variables conocidas como variables de Ashtekar-Barbero. Estas variables surgen al escribir la métrica espacial en términos de triadas y a partir de ellas construir una tripleta de variables análogas a campos eléctricos $\vec{E}_{i}$ cuyos momentos conjugados están dados por una tripleta de conexiones análogas a los potenciales vectoriales $\vec{A}_{i}$. El espacio fase en estas variables es análogo al de una teoría $S U(2)$ de Yang-Mills, una generalización no abeliana del electromagnetismo. Las variables de conexión $\overrightarrow{A_{i}}$ proveen una definición de transporte paralelo de los espinores de $\mathrm{SU}(2)$ sobre la variedad espacial, a través de las holonomías

$$
h_{e}[A]=p \exp \int_{e} \vec{A} \cdot d \vec{s}
$$

Las variables $\vec{E}_{i}$ tienen una nueva interpretación física, ellas codifican la geometría del espacio tridimensional. Más precisamente, definen en cada punto del espacio un marco de referencia local que puede usarse para reconstruir la métrica espacial. Todas las propiedades geométricas del espacio se escriben como funcionales de $\vec{E}_{i}$. En particular el área $\mathcal{A}(S)$ de una superficie bidimensional y el volumen $\mathcal{V}(R)$ de una

\footnotetext{
${ }^{1}$ Para una introducción conceptual ver [229], los ingredientes básicos se pueden consultar en [230], para aspectos más generales ver [147], los fundamentos matemáticos se exponen en [148].
} 
región tridimensional son funcionales de $\vec{E}_{i}$. Las ecuaciones de Einstein están codificadas en relaciones entre las variables $\vec{E}$ y $\vec{A}$ : las llamadas constricciones cinemáticas y la constricción Hamiltoniana.

Para cuantizar la teoría se sigue el esquema canónico, es decir, las variables se promueven a operadores en cierto espacio de Hilbert que satisfacen las relaciones de conmutación canónicas cambiando paréntesis de Poisson por conmutadores. Las constricciones clásicas ahora serán ecuaciones de operadores sobre estados. La versión cuántica de (E.1) es un operador que actúa en el estado de vacio para dar lugar a ciertas excitaciones unidimensionales llamadas estados de lazo. La construcción del espacio de Hilbert de la gravedad cuántica comienza considerando un conjunto estados de múltiples lazos. Estos estados pueden combinarse para formar una base ortonormal, los estados de esa base son conocidos como estados de red de espín. Las redes de espín son eigenestados de la geometría (Ver figura E.1).

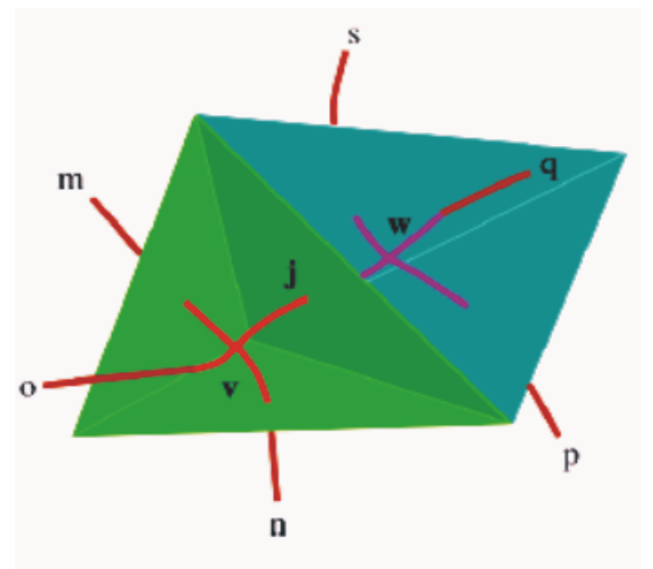

Figura E.1: Los estados de la base ortonormal del espacio de Hilbert se conocen como estados de red de espin. Estos estados están representados por gráficos con números cuánticos asignados a las aristas e intersecciones del gráfico.

Dada una superficie bidimensional se define un operador asociado con el área. Resulta que el gráfico de un estado de red de espín que atraviesa la superficie en ciertos puntos es un eigenestado del operador área cuyos eigenvalores son proporcionales al cuadrado de la longitud de Planck; lo mismo ocurre con el volumen (Ver figura E.2). Por lo tanto, esta teoría predice una cuantización de la geometría. Una de las implicaciones más importantes de esta geometría discreta es la elusión de singularidades.

\section{E.2.2. Geometría Cuántica}

El espacio fase de la teoría está formado por pares $(A, E)$, donde $A_{a}^{i}$ son conexiones que toman valores en el álgebra de Lie del grupo de estructura. Los campos eléctricos $E_{i}^{a}$ son vectores valuados en el dual del algebra de Lie, en el caso de la gravedad cuántica el grupo es $S U(2)$. Se toman como observables las holonomías $h_{e}$ definidas por $A$ a lo largo de trayectorias, y los flujos $F_{S}$ de los campos eléctricos $E$ a través de superficies bidimensionales $S$. El espacio de Hilbert de la gravedad cuántica puede construirse 


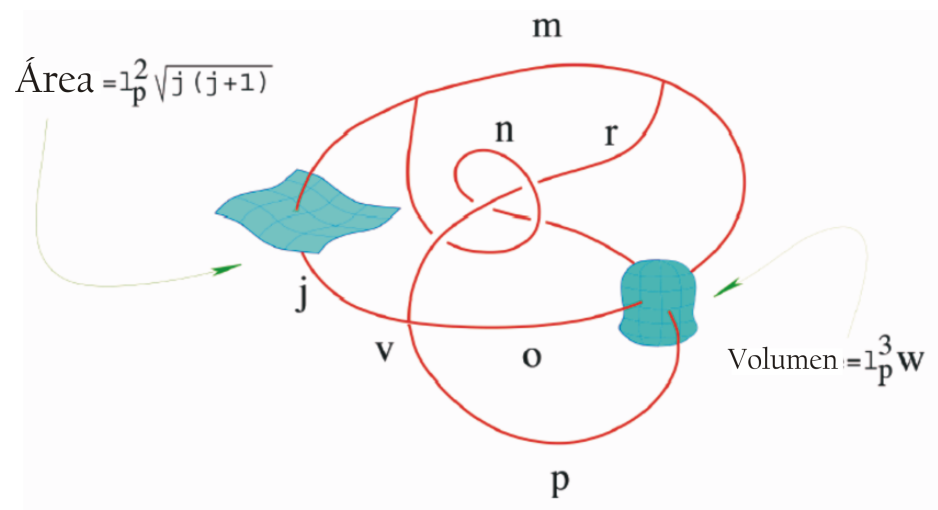

Figura E.2: Las aristas de las redes de espín son líneas que representan cuantos de área. Una red de espín asociada a un gráfico incluyendo una arista de etiqueta $j$ que atraviesa una superficie dada es un eigenestado del área de esa superficie. Las intersecciones etiquetan números cuánticos de volumen.

de dos maneras. La primera es a través de una construcción GNS. La segunda es comenzar especificando un espacio adecuado para las funciones de las conexiones: $C y l$. Se fija una gráfica $\gamma$ con $N$ aristas en una variedad tridimensional. La conexión $A$ asocia a cada arista $e$ una holonomía $h_{e}$. El espacio formado por $\left(h_{1}, \ldots, h_{N}\right)$ define la configuración $h_{\gamma}$ restringida a una gráfica $\gamma$. Dada una función compleja suave $\psi$, se puede definir una función de las conexiones como

$$
\Psi(A)=\psi\left(h_{1}, \ldots, h_{N}\right)
$$

El espacio de estas funciones se denota como $C y l_{\gamma}$. Los elementos de $C y l_{\gamma}$ sólo saben de las conexiones restringidas a $\gamma$. El espacio de todas las funciones cilíndricas se obtiene al considerar todas las posibles gráficas $\gamma$.

$$
C y l=\bigcup_{\gamma} C y l_{\gamma}
$$

Los elementos de $C y l$ sólo dependen de las holonomías de las conexiones a lo largo de las aristas de alguna grafica $\gamma$. Al restringirse a una sola gráfica $\gamma$, la teoría puede verse análoga a una teoría de norma en una red irregular. Sin embargo, como deben de considerarse todas las posibles gráficas se trata entonces de una teoría de campo con un número infinito de grados de libertad de todas las conexiones.

El siguiente paso es introducir el producto interno en $C y l$, dadas $\Psi_{1}, \Psi_{2} \in C y l$ como

$$
\left(\Psi_{1}, \Psi_{2}\right)=\int_{A_{\gamma}} \bar{\psi}_{1} \psi_{2} d \mu_{H}^{(N)}
$$

donde $\mu_{H}^{(N)}$ es la medida de Haar. La cerradura de Cauchy de Cyl respecto a este producto interno proporciona el espacio de Hilbert $\mathcal{H}_{L Q G}$.

Como en la construcción se consideran todas las posibles gráficas $\gamma$, el espacio $\mathcal{H}_{L Q G}$ es muy grande. No obstante puede descomponerse en subespacios asociados con las etiquetas $j_{e}$ de las aristas de cada una de 
las gráficas $\gamma$, de tal modo que $\mathcal{H}_{L Q G}$ se escribe

$$
\mathcal{H}_{L Q G}=\bigoplus_{\gamma, \vec{j}} \mathcal{H}_{\gamma, \vec{j}}
$$

a esta descomposición se le conoce como la descomposición en redes de espín. Al ser $\mathcal{H}_{L Q G}$ muy grande, los cálculos prácticos son viables al realizarse en los subespacios $\mathcal{H}_{\gamma, \vec{j}}$. Además muchos de los operadores físicamente relevantes en $\mathcal{H}_{L Q G}$ como las holonomías, los flujos, el área y el volumen aparecen como familias de operadores en $C y l_{\gamma}$. Por lo tanto, sus propiedades pueden ser exploradas en términos de sus acciones en los espacios de dimensión finita $\mathcal{H}_{\gamma, \vec{j}}$.

Estas estructuras son suficientes para describir la cinemática cuántica. Sin embargo, el caso de la dinámica cuántica es más complicado ya que las soluciones a las ecuaciones de Einstein no pertenecen al espacio de Hilbert $\mathcal{H}_{L Q G}$, al no ser normalizables. Sucede que las soluciones se encuentran en el espacio $C y l^{*}$, que es el espacio dual a $C y l$. Así se tiene la estructura del espacio de Hilbert como la tripleta

$$
C y l \subset H_{L Q G} \subset C y l^{*} .
$$

Los estados físicos se encuentran en $C y l^{*}$. Sin embargo, no se ha encontrado una estructura de espacio de Hilbert en $C y l^{*}$.

\section{E.2.3. Cosmología Cuántica de Lazos}

\section{Cosmología Clásica}

Según el modelo de la gran explosión ó Big Bang en etapas muy tempranas del universo el espacio era tan pequeño y la densidad de energía tan grande que, durante la subsecuente expansión, el universo no se pudo haber diluido hasta llegar a ser como lo indican las observaciones actuales. Para explicar la estructura actual se introduce en el modelo la fase inflacionaria que es una expansión acelerada en etapas muy tempranas en la vida del universo. Los rastros de esta fase pueden observarse en las anisotropías de la radiación cósmica de fondo, las cuales están en completo acuerdo con las predicciones teóricas.

Sin embargo, el esquema aún tiene ciertos problemas, por ejemplo, el campo inflacionario debe introducirse a mano y sus características deben ser muy particulares, las densidades de energía divergentes y la singularidad espacial primordial son problemas sobre lo que la teoría no puede dar conclusiones definitivas.

En la cosmología estándar se asume que el espacio es homogéneo e isotrópico, lo cual es una muy buena aproximación para grandes escalas actualmente. La métrica del espacio es determinada por el factor de escala $a(t)$ que da el radio del universo a un tiempo dado. Esta función describe la expansión del universo de acuerdo a la ecuación de Friedmann

$$
\left(\frac{\dot{a}}{a}\right)^{2}=\frac{8 \pi}{3} G \rho(a),
$$


que proviene de las ecuaciones de Einstein bajo la suposición de isotropía, en (E.7) $\rho(a)$ es la densidad de energía de la materia en el universo. Para la radiación, por ejemplo, $\rho \propto a^{-4}$, en este caso la ecuación de Friedmann tiene como solución $\dot{a} \propto a^{-1}$ y $a(t) \propto \sqrt{t-t_{0}}$, con $t_{0}$ una constante de integración. Para cualquier solución existe un tiempo $t=t_{0}$ para el cual el tamaño del espacio desaparece y la $\rho$ diverge. Lo único que la teoría puede decir al respecto es que existe una singularidad.

En analogía al problema de la singularidad en gravitación, existe un problema de estabilidad en el átomo de hidrógeno donde el electrón caería al núcleo en un tiempo muy corto. Este problema es resuelto en mecánica cuántica ya que la energía del estado base en finita, lo que implica que el electrón no puede radiar toda su energía y por tanto no caerá al haber alcanzado el estado base. En el límite $\hbar \rightarrow 0, E_{0} \rightarrow-\infty$ se regresa al caso de la singularidad clásica. Se espera que en cosmología pase algo similar a lo que sucede con el electrón del átomo de hidrógeno, en escalas del orden de la escala de Planck $\ell_{p}$. Si las densidades

están acotadas por $\ell_{p}^{-3}$, estas serán finitas en el marco de la gravedad cuántica pero serán divergentes en la teoría clásica.

Por otro lado, considerando el modelo cosmológico de Friedmann-Robertson-Walker, se puede mostrar matemáticamente que el universo no puede estar en equilibrio térmico [25]. Sin embargo, para propósitos prácticos el universo en gran parte de su historia ha estado muy cerca del equilibrio y sólo en etapas muy tempranas las desviaciones del equilibrio fueron muy importantes. La ecuación de Boltzmann relativista se introduce al querer estudiar esas épocas donde todas las especies que existían eran relativistas.

La historia térmica del universo [28] puede entenderse como la competencia entre la rapidez de la expansión del universo y la rapidez de en que las partículas interactúan, tal que, en buena aproximación se tiene que la temperatura del universo decrece como el inverso del factor de escala. Así las épocas en el universo se entienden según la temperatura y la cantidad de reacciones que existieron: En épocas más tempranas el universo era un plasma de partículas relativistas $T>10^{16} \mathrm{GeV}$, a temperaturas entre $10^{14}$ y $300 \mathrm{GeV}$ ocurre una transición de fase que propicia rompimiento de simetrías (como el mecanismo de Higgs y la transición electrodébil). A una temperatura de $100-300 \mathrm{MeV}$ se producen los bariones y a $10-0.1 \mathrm{MeV}$ se da la nucleosíntesis y se comienzan a desacoplar las especies hasta que la radiación se desacopla de la materia a una temperatura aproximada de $0.27 \mathrm{eV}$, es decir la temperatura del fondo cósmico de microondas. Es claro que para temperaturas mayores a $10^{19} \mathrm{GeV}$ debe ser la época dominada por efectos cuánticos relativistas.

\section{Cosmología Cuántica}

Comenzando con la ecuación de Friedmann (E.7) y reemplazando $\dot{a}$ por el momento conjugado $p_{a}=$ $3 a \dot{a} / 8 \pi G$, el Hamiltoniano resultante es cuadrático en el momento y puede ser cuantizado fácilmente convirtiéndose en un operador que actúa sobre una función de onda que depende de $a$ y posiblemente de algún campo de materia $\phi$. Al igual que en la mecánica cuántica, los operadores básicos se definen por multiplicación y diferenciación. Esto lleva a la llamada ecuación de Wheeler-DeWitt 


$$
\frac{3}{2}\left(-\frac{1}{9} \ell_{p}^{4} a^{-1} \frac{\partial}{\partial a} a^{-1} \frac{\partial}{\partial a}\right) a \psi(a, \phi)=8 \pi G \hat{H}_{\phi}(a) \psi(a, \phi),
$$

en donde $\hat{H}_{\phi}(a)$ es el Hamiltoniano de materia. Este sistema difiere de los sistemas cuánticos usuales en que la ecuación (E.8) no contiene derivadas temporales. Esto es consecuencia de la covariancia general. El Hamiltoniano no da más una ecuación de evolución, ahora es una constricción que restringe los estados permitidos $\psi(a \phi)$. No obstante, (E.8) se puede interpretar como una ecuación de evolución en el parámetro $a$, que ahora se llamará tiempo interno, la evolución de la materia se mide ahora en relación a la expansión del universo. Sin embargo, esta cuantización también lleva a densidades divergentes en $a=0$, que además sigue siendo un punto singular. Esto sucede ya que la cuantización que sigue la ecuación de WheelerDeWitt es la misma que la de la mecánica cuántica de Schrödinger. El problema radica en este tipo de cuantización.

\section{Variables de lazos en Cosmología cuántica}

Como se vio en el capítulo 5, una cuantización no perturbativa e independiente del fondo se encuentra en la gravedad cuántica de lazos, donde las variables dinámicas (gravitacionales) son las conexiones de espín $S U(2)$ y sus momentos conjugados, las triadas densitizadas análogas a los campos eléctricos. Estas variables permiten una representación independiente de la métrica de fondo y son integradas en holonomías y flujos. La base ortonormal de la representación de lazos es la llamada base de redes de espín. Esta cuantización difiere de la cuantización tipo Schrödinger que se usó en la ecuación de Wheeler-DeWitt donde la métrica es usada como variable básica y cuantizada como en mecánica cuántica. Los flujos y las holonomías actúan como operadores bien definidos y los flujos tienen espectro discreto. Como la geometría es determinada por las triadas, entonces la geometría es también discreta.

En el caso particular de los modelos isotrópicos, se reformula el problema en términos de triadas y conexiones en lugar de la variable $a$ [232]-[238]. Las variables canónicas se parametrizan con las variables $c$ y $p$

$$
A_{a}^{i}=c \ell^{-1} \omega_{a}^{i}, \quad E_{i}^{a}=p \ell^{-2} \sqrt{q} e_{i}^{a}
$$

donde $q$ es la 3-métrica de referencia, $\ell \propto V^{1 / 3}$ es el volumen en la métrica de referencia, y $e, \omega$ son los vectores y uno-formas (duales de los vectores) base de la métrica de referencia, es decir $q_{a b}=\omega_{a}^{i} \omega_{b}^{j} \mathcal{K}_{i j}$, con $\mathcal{K}_{i j}$ la métrica de Cartan en $\mathrm{su}(2)$.

El papel del factor de escala le corresponde a las triadas $p$ con $|p|=a^{2}$ cuyos momentos conjugados son las conexiones isotrópicas $c=-\dot{a} / 2$. Al ser $A$ y $E$ pares conjugados, el paréntesis de Poisson entre $c$ y $p$ se calcula directamente y es $\{c, p\}=8 \pi G / 3$. La variable $p$ puede tomar valores con ambos $\operatorname{signos}, \operatorname{sgn}(p)$ se entiende como la orientación del espacio. A partir de $c$ y $p$ se construyen las correspondientes holonomías y flujos que generan un álgebra la cual será promovida a un álgebra de operadores.

Así los estados en esta representación se escriben como funciones de holonomías, los estados ortonormales 
son funciones de las conexiones isotrópicas. En esos estados las variables básicas actúan de la siguiente manera

$$
\begin{aligned}
\hat{p}|\mu\rangle & =\frac{1}{6} \ell_{p}^{2} \mu|\mu\rangle, \\
\widehat{e^{i \mu^{\prime} c / 2}}|\mu\rangle & =\left|\mu+\mu^{\prime}\right\rangle . \\
{\left[\widehat{e^{i \mu^{\prime} c / 2}}, \hat{p}\right] } & =\left[\widehat{e^{i \mu^{\prime} c / 2}}, \hat{p}\right] .
\end{aligned}
$$

El operador $\hat{p}$ tiene espectro discreto, además sólo se representan exponenciales $\widehat{e^{i \mu^{\prime} c / 2}}$ y no $\hat{c}$ directamente. La relación de conmutación es (E.12).

Los operadores exponenciales como (E.11) no son débilmente continuos en $\mu^{\prime}$, ya que todos los estados son ortogonales, es decir, $\left\langle\mu\left|\widehat{e^{i \mu^{\prime} c / 2}}\right| \mu^{\prime}\right\rangle=\delta_{0, \mu^{\prime}}$. Esto implica que el espacio de Hilbert en este caso no es separable y por lo tanto la cuantización por lazos no es equivalente a la cuantización de Wheeler-DeWitt. No obstante, el espacio de Hilbert de la cuantización por lazos puede escribirse como un espacio de funciones cuadrado integrables, no sobre los reales, sino en un espacio conocido como la compactificación de Bohr.

\section{Dinámica y efectos cuánticos}

La dinámica en el caso cosmológico se estudiará a partir de la constricción Hamiltoniana que se impone sobre ciertos estados de la forma $|\psi\rangle=\sum_{\mu} \psi_{\mu}|\mu\rangle$, donde $|\mu\rangle$ son eigenestados del operador triada, los coeficientes dependen también de los campos de materia $\phi$. Como no existe un operador $\hat{c}$ es necesario utilizar en su lugar al operador exponencial (E.11). Entonces al cuantizar (E.7) se obtiene la siguiente ecuación en diferencias finitas

$$
\begin{aligned}
& \left(V_{\mu+5}-V_{\mu+3}\right) \psi_{\mu+4}(\phi)-2\left(V_{\mu+1}-V_{\mu-1}\right) \psi_{\mu}(\phi)+ \\
& \left(V_{\mu-3}-V_{\mu-5}\right) \psi_{\mu-4}(\phi)=-\frac{4}{3} \pi G \ell_{p}^{2} \hat{H}_{m a t}(\mu) \psi_{\mu}(\phi),
\end{aligned}
$$

donde $V_{\mu}=\left(\ell_{p}^{2}|\mu| / 6\right)^{3 / 2}$ son los eigenvalores de volumen provenientes del operador $\hat{V}=|\hat{p}|^{3 / 2} \mathrm{y}$ el Hamiltoniano de materia $\hat{H}_{\text {mat }}$. De la misma forma que la ecuación (E.8), (E.13) es una constricción ya que no genera evolución en la coordenada temporal sino en un tiempo interno etiquetado con el índice $\mu$. Una diferencia importante entre (E.8) y (E.13) es que en la última ecuación la evolución en el tiempo interno no se rompe en la singularidad clásica sino que continua a través de ella, para $\mu$ negativas $V_{\mu}$ decrece, mientras que para $\mu$ positivas $V_{\mu}$ crece. Esto da la idea de un universo que colapsa hasta que alcanza una longitud mínima que corresponde a la singularidad clásica y entonces vuelve a expandirse.

En el caso clásico el Hamiltoniano de materia es de la forma $H_{m a t}=8 \pi G a^{-3} p_{\phi}^{2}$, hay que cuantizar $a^{-3}$. En el caso de Wheeler-DeWitt el operador $a^{-3}$ no está acotado y diverge en la singularidad clásica. En la cuantización por lazos se puede reescribe como

$$
a^{-3}=\left(\frac{1}{\pi G} \operatorname{Tr} \tau_{3} e^{c \tau_{3}}\left\{e^{-c \tau_{3}}, \sqrt{V}\right\}\right)^{6}
$$


donde $\tau_{3}$ son matrices relacionadas con las matrices de Pauli, y el último término a la izquierda es el paréntesis de Poisson. En esta expresión sólo se necesitan potencias positivas del operador $\hat{p}$. La cuantización de (E.14) define el siguiente operador

$$
\widehat{a^{-3}}=\left[8 i \ell_{p}^{-2}\left(\operatorname{sen} \frac{c \sqrt{\hat{V}}}{2} \cos \frac{c}{2}-\cos \frac{c \sqrt{\hat{V}}}{2}\right) \operatorname{sen} \frac{c}{2}\right]^{6}
$$

este operador es finito y su acción sobre los estados es

$$
\widehat{a^{-3}}|\mu\rangle=\left[4 \ell_{p}^{-2}\left(\sqrt{V_{\mu+1}}-\sqrt{V_{\mu-1}}\right)\right]^{6}|\mu\rangle .
$$

En particular cuando $\mu=0$, en la singularidad clásica, el operador $\widehat{a^{-3}}$ no diverge aunque es cero. De esta forma el Hamiltoniano de materia tampoco es singular

$$
\hat{H}_{m a t}=\frac{1}{2} \widehat{a^{-3}} \hat{p}_{\phi}^{2}+\hat{V} V(\phi) .
$$

El carácter finito del operador Hamiltoniano es una consecuencia de la representación de lazos. Sin embargo, la reescritura (E.14) no es la única posible para $a^{-3}$ desde el punto de vista clásico, por lo que pueden existir ciertas ambigüedades al momento de cuantizar. Ciertas propiedades importantes se mantienen bajo estas ambigüedades, no obstante, ciertos detalles finos pueden cambiar. Dos parámetros caracterizan estas ambigüedades, $j$ de la representación del grupo de holonomías y $l$ que es la potencia de $|p|$ en los paréntesis de Poisson. Los eigenvalores resultantes se pueden aproximar por

$$
a_{e f}^{-3}=a^{-3} D_{l}\left(\frac{a^{2}}{a_{\max }^{2}}\right)^{\frac{3}{2-2 l}}
$$

donde $a_{\max }=\ell_{p} \sqrt{j / 3}$ y la función $D_{l}$ es

$$
D_{l}(q)=\frac{3}{2 l} q^{1-l}\left[\frac{(q+1)^{l+2}-|q-1|^{l+2}}{(l+2)}-q \frac{(q+1)^{l+1}-\operatorname{sgn}(q-1)|q-1|^{l+1}}{(l+1)}\right] .
$$

Con esto se puede ver que la función $a_{e f}^{-3}$ tiene tres regímenes, se acerca al valor clásico $a^{-3}$ para grandes escalas $a \gg a_{\max }$, tiene un máximo alrededor de $a=a_{\max } \mathrm{y}$ decae para $a \ll a_{\max }$.

Ya que la ecuación (E.13) es complicada de estudiar, es útil trabajar con ecuaciones efectivas que surgen de diferentes tipos de correcciones a la constricción Hamiltoniana. La ecuación de Friedmann efectiva es

$$
a \dot{a}^{2}=\frac{8 \pi}{3} G\left[\frac{1}{2} a_{e f}^{-3} p_{\phi}^{2}+a^{3} V(\phi)\right] .
$$

Como el Hamiltoniano de materia no sólo actúa como fuente del campo gravitacional, sino que da las ecuaciones de movimiento, las modificaciones en (E.20) cambian también las ecuaciones de movimiento de la materia; así, la ecuación de Klein-Gordon efectiva es

$$
\ddot{\phi}=\dot{\phi} \dot{a} \frac{d}{d a} \ln \left(a_{e f}^{-3}\right)-a^{3} a_{e}^{-3} f V^{\prime}(\phi),
$$


estas ecuaciones pueden estudiarse numéricamente.

La ecuación de Friedmann efectiva (E.20) se comporta diferente a escalas pequeñas ya que aumenta con $a$ para $\phi$ y $p_{\phi}$ fijos. Se puede analizar (E.20) como una ecuación análoga a la energía con $\dot{a}^{2}$ como el término cinético y como potencial a $\mathcal{V}(a)=-(8 \pi G / 3 a)\left(a_{e f}^{-3} p_{\phi}^{2} / 2+a^{3} V(\phi)\right)$. La parte dominante de este potencial se comporta como $-a^{-4}$ que va en aumento lo que demuestra que $a$ será llevado hacia valores más pequeños, lo que implica la atracción en el universo.

Por debajo del máximo de la densidad eficaz $\mathcal{V}(a)$ decae, mientras $-\mathcal{V}(a)$ evoluciona como una potencia positiva de $a$. Esto implica que el factor de escala será repelido de $a=0$ de forma tal que, a pequeñas escalas, hay una componente repulsiva a la gravitación provocada por efectos cuánticos. De esta forma se previene el colapso gravitacional. Esto ocurre a través de rebotes donde a pasa de un comportamiento de contracción a una de expansión, las condiciones para que se lleve a cabo son claramente $\dot{a}=0 \mathrm{y}$ $\ddot{a}>0$. Existen dos posibilidades de rebotes, la primera es si el espacio tenga curvatura positiva en lugar de ser finito y la segunda con un potencial que pueda ser negativo, sin embargo, esto siempre corresponde a un máximo en lugar de un mínimo. No obstante, con las modificaciones en (E.20) el término extra proporciona una contribución positiva que puede volverse suficientemente grande para que $\ddot{a}$ se vuelva positiva y crear un rebote. La presencia de estos depende del modelo particular que se estudie. En el caso de la singularidades de la gran explosión y el gran colapso, estas son reemplazadas por un gran rebote cuántico que evade la singularidad, estos rebotes tienen lugar a un volumen mínimo de aproximadamente $1.28 \times 10^{33} \mathrm{~cm}$.

Esta contribución repulsiva no sólo puede explicar la ausencia de singularidades sino que también aumenta la expansión del universo cerca de la singularidad. Entonces, según el modelo, el universo acelera a partir de efectos cuánticos, es decir, proporciona un mecanismo de inflación sin introducir un tipo de materia específica. A través de la formación de estructura, la fase inflacionaria puede dejar una huella en el fondo cósmico de microondas. La inflación de lazos puede distinguirse del inflatón usual ya que la potencia depende de forma diferente en cada escala.

La dinámica modificada de la materia se vuelve importante ya que a escalas pequeñas el término de fricción cambia de signo y la materia aumenta su potencial si tiene un momento inicial diferente de cero por pequeño que este sea. Después de esa fase la materia se frena y regresa a su mínimo proveyendo más inflación. Cuando llega al mínimo comienza a oscilar y así puede comenzar el habitual y re-calentamiento para obtener la materia caliente. La inflación por lazos provee una alternativa a los modelos de inflación usuales que, como se sabe, concuerdan con las observaciones. 
E.2. Gravedad Cuántica de Lazos 


\section{Bibliografía}

[1] F. Jüttner, Ann. Physik und Chemie 34, 856 (1911); F. Jüttner, Ann. Physik und Chemie 35, 145 (1911); F. Jüttner, Zeitschr. Phys. 47, 542 (1928).

[2] A. G. Walker, Proc. Edinburgh Math. Soc. 2, 238 (1934-36).

[3] A. Lichnerowicz and R. Marrot, Compt. Rend. Acad. Sci. 210, 759 París (1940). R. Marrot, J. Math Pures et Appl. 25, 93 (1946).

[4] A. H. Taub, Relativistic Rankine- Hugoniot Equiation. Phys. Rev. 74, 328-334 (1948).

[5] J. L. Synge, The Relativistic Gas. North-Holland Publishing Company, Amsterdam (1957).

[6] W. Israel, Relativistic Kinetic Theory of a simple gas. J. Math. Phys. 4, 1163-1181 (1963).

[7] D. C. Kelly, The Kinetic Theory of a Relativistic Gas. Unpublished Report. Miami University, Oxford (1963).

[8] N. A. Chernikov, The Relativistic Gas in the Gravitational Field. Acta Phys. Pol. 23 629-645 (1963).

[9] R. W. Lindquist, Relativistic Transport Theory. Annals of Physics 37, 487-518 (1966).

[10] J. M. Stewart, Non-equilibrium relativistic kinetic theory. Lecture Notes in Physics Vol. 10 Springer, Heidelberg (1971).

[11] J. Ehlers, General Relativity and Kinetic Theory en los Proceedings of the International School of Physics, Enrico Fermi pág. 1-70, Ed. R. K. Sach, Academic Press (1971).

[12] S. R. de Groot, W. A. van Leewen and Ch. G. van Weert, Relativistic Kinetic Theory. North-Holland, Amsterdam (1980). 
[13] C. Cercignani and G. M. Kremer, The Relativistic Boltzmann Equation: Theory and Applications. Progress in Mathematical Physics Vol. 22 Springer-Verlag, Birkhäuser, Basel, (2002).

[14] L. P. Horwitz, S. Shashoua, and W. C. Schieve, Physica A 161, 300 (1989).

[15] E. Lehmann, J. Math. Phys. 47, 023303 (2006).

[16] J. Dunkel, P. Talkner and P. Hänggi, New J. Phys. 9, 144 (2007).

[17] J. Dunkel and P. Hänggi, Phys. Rev. E 71, 016124 (2005).

[18] A. Sandoval-Villalbazo, A. L. García-Perciante and L. S. García-Colín, AIP Conf. Proc. 1122, 388 (2009).

[19] D. Cubero, J. Casado-Pascual, J. Dunkel, P. Talkner and P. Hänggi, Phys. Rev. Lett. 99, 170601 (2007).

[20] A. Montakhab, M. Ghodrat, and M. Barati, Phys. Rev. E 79, 031124 (2009).

[21] A. Aliano, L. Rondoni and G. P. Morriss, Eur. Phys. J. B 50, 361 (2006).

[22] F. Peano, M. Marti, L. O. Silva and G. Coppa, Phys. Rev. E 79, 025701(R) (2009).

[23] J. Dunkel, P. Hänggi and S. Hilbert, Nature Phys. 5, 741 (2009).

[24] S. Chandrasekhar, An Introduction to the Study of Stellar Structure. Dover Publications Inc. (1958).

[25] J. Bernstein, Kinetic Theory in the Expanding Universe. Cambridge University Press, Paperback Ed. (2004).

[26] A. R. Liddle, D. H. Lyth, Cosmological Inflation and Large Scale Structure. Cambridge University Press (2000).

[27] J. A. Peacock, Cosmological Physics. Cambridge University Press (1999).

[28] E. W. Kolb, M. S. Turner, The Early Universe. Paperback Ed. Westview Press (1994).

[29] L. García-Colín Scherer, Introducción a la Física Estadística. El Colegio Nacional, México (2005).

[30] L. García-Colín Scherer y P. Goldstein Menache, La Física de los Procesos Irreversibles. Vol I y Vol. II. El Colegio Nacional México (2003).

[31] R. A. Sunyaev and Y.B. Zeldovich, Comments Astrophys. Space Phys. 4, 173 (1972); M. Jones et al., Nature (London) 365, 320 (1993); M. Birkinshaw, S. F. Gull, and H. Hardebeck, Nature (London) 309, 34 (1984). 
[32] N. Itoh, Y. Kohyama, S. Nozawa, Relativistic corrections to the Sunyaev-Zeldovich effect for clusters of galaxies. Astrophys. J. 502, 7-15 (1998).

[33] Z. Haba, Linearized Kompaneetz equation as a relativistic diffusion. Mod. Phys. Lett. A 24, 3193-3203 (2009).

[34] P. Huovinen and D. Molnar, Phys. Rev. C 79, 014906 (2009); W. Florkowski, W. Broniowski, M. Chojnacki and A. Kisiel, Acta Phys. Polon. B 401093 (2009).

[35] H. van Hees, V. Greco, and R. Rapp, Phys. Rev. C 73, 034913 (2006).

[36] H. B. Callen, Themodynamics and an Introduction to Thermostatistics. John Wiley and Sons Inc. (1985).

[37] M. Montesinos and C. Rovelli, Class. Quantum Grav. 18, 555 (2001).

[38] H. Quevedo, J. Math Phys. 48013506 (2007); H. Quevedo, Gen. Relativ. Gravit. 40 971-984 (2008); H. Quevedo, A. Sánchez, S. Taj and A. Vázquez, Gen. Relativ. Gravit. DOI: 10.1007/s10714-0100996-2 (2010); A. Vázquez, H. Quevedo and A. Sánchez, J. Geom. Phys. 60 1942-1949 (2010).

[39] R. M. Wald, Quantum Field Theory in Curved Spacetime and Black Hole Thermodynamics. The University of Chicago Press (1994).

[40] C. Kiefer, Quantum Gravity: General Introduction and Recent Developments. gr-qc/0508120 (2005).

[41] F. Dowker, R. D. Sorkin and J. Henson, Quantum Gravity Phenomenology, Lorentz Invariance and Discreteness. Mod. Phys. Lett. A19 1829-1840 (2004).

[42] B. L. Hu and E. Verdaguer, Liv. Rev. Rel. 11, 3 (2008). (http://www.livingreviews.org/lrr-2008-3).

[43] M. Rangamani, Gravity \& Hydrodinamics: Lectures on the fluid gravity correspondence. arxiv:0905.4352.

[44] E. Verlinde, On the origin of gravity and the laws of Newton. arxiv:1001.0785.

[45] T. Jacobson, Phys. Rev. Lett. 75 1260-1263 (1995).

[46] G. Chacón-Acosta, L. Dagdug and H. Morales-Técotl, Manifestly covariant Jüttner distribution and equipartition theorem, Phys. Rev. E 81, 021126 (2010).

[47] G. Chacón-Acosta, L. Dagdug and H. A. Morales-Técotl, On the Fokker-Planck equation for the relativistic Lorentz gas, in New Trends in Statistical Physics: Festschrift in Honor of Leopoldo GarciaColin's 80th Birthday, ed. A. Macías and L. Dagdug, (pp. 275-292 World Scientific, 2010). 
[48] G. Chacón-Acosta, L. Dagdug and H. Morales-Técotl, Relativistic Momentum and Manifestly Covariant Equipartition Theorem Revisited, in Gravitational Physics: Testing Gravityfrom Submillimeter to Cosmic Scale, ed. H. Morales-Técotl, L. A. Ureña-López, R. Linares-Romero and H. H. GarcíaCompean, AIP Conf. Proc. 1256, 231-238 (2010).

[49] G. Chacón-Acosta, L. Dagdug and H. Morales-Técotl, A covariant Fokker-Planck equation for a simple gas from relativistic kinetic theory, in IV Mexican Meeting on Theoretical and Experimental Physics: Relativistic Fluids and Biological Physics, Ed. L. Dagdug, A. L. García-Perciante, A. Sandoval-Villalbazo, L. S. García-Colin, AIP Conf. Proc. 1312, 73-79 (2010).

[50] G. Chacón-Acosta, E. Manrique, L. Dagdug and H. Morales-Técotl, Statistical thermodynamics of simple polymer quantum systems, (en preparación).

[51] C. Eckart Thermodynamics of Irreversible Processes III. Relativistic Theory of the Simple Fluid, Phys. Rev. 58, 919-924 (1940).

[52] L. D. Landau y E. M. Lifshitz, Mecánica de Fluidos. Editorial Reverté, S. A. 2a. Reimpresión (2001).

[53] L. H. Ryder, Quantum Field Theory, 2nd Ed. Cambridge University Press, (1996).

[54] B. F. Schutz, A First Course in General Relativity, Cambridge University Press, (1990).

[55] J. L. Synge, Relativity: The Special Theory. North-Holland Publishing Company, (1972).

[56] L. D. Landau and E. M. Lifshitz, The Classical Theory of Fields, 4th ed. Pergamon Press, Oxford (1980).

[57] R. Hagedorn, Relativistic Kinematics, W. A: Benjamin Inc. (1963).

[58] E. C. G. Sudarshan and N. Mukunda Classical Dynamics. A Modern Perspective. John Wiley \& Sons (1974).

[59] D. G. Currie, T. F. Jordan, E. C. G. Sudarshan, Rev. Mod. Phys. 35, 350 (1963).

[60] G. Marmo, N. Mukunda, E. C. G. Sudarshan, Phys. Rev. D 30, 2110 (1984).

[61] F. Rohrlich, Ann. Phys. 117, 292 (1979).

[62] H. van Dam and E. P. Wigner, Rev. Mod. Phys. 37, 595 (1965).

[63] G. Esposito, G. Marmo, G. Sudarshan, From Classical to Quantum Mechanics. Cambridge university Press (2004).

[64] A. Mangeney, Physica 30, 461 (1964). 
[65] R. Balescu and T. Kotera, Physica 33, 558 (1967).

[66] C. Tian, Manifestly covariant classical correlation dynamics I. Gerneral theory, Ann. Phys. (Berlin) 18, 783-808 (2009); C. Tian, Manifestly covariant classical correlation dynamics II. Transport equations and Hakim equilibrium conjeture, Ann. Phys. (Berlin) 19,75-101 (2010).

[67] S. Weinberg, Cosmology (Oxford University Press, 2008).

[68] M. Abramowitz and I. Stegun, Handbook of Mathematical Functions. Dover (1968).

[69] A. Einstein, Jahrb. Radioakt. Elektron. 4, 411 (1907); M. Planck, Ann. Physik 26, 1 (1908); H. Ott, Z. Physik 175, 70 (1963); A. Arzelies, Nuovo Cimento 35, 792 (1965); P. T. Landsberg, Nature (London) 212, 571 (1966).

[70] P. T. Landsberg, Nature (London) 214, 903 (1967).

[71] N. G. van Kampen, Physica (Utrecht) 43, 244 (1969).

[72] G. Neugebauer, Relativistische Thermodynamik (Akademic-Verlag, Berlin, 1980).

[73] P. A. M. Dirac, Proc. R. Soc. A 106, 581 (1924).

[74] F. Debbasch, J. P. Rivet and W. A. van Leeuwen, Physica A 301, 181 (2001).

[75] C. W. Misner, K. S. Thorne and J. A. Wheeler, Gravitation. W. H. Freeman and Company New York (1973).

[76] D. Cubero and J. Dunkel, EuroPhys. Lett. 87, 30005 (2009).

[77] F. Debbasch, Physica A 387, 2443 (2008).

[78] R. C. Tolman, Phil. Mag. (Series 6), 28, 583-600 (1914); R. C. Tolman, Phys. Rev. 11, 261 (1918).

[79] A. L. García-Perciante, A. Sandoval-Villalbazo and L. S. García-Colín, Physica A 387, 5073 (2008).

[80] S. Weinberg, Gravitation and Cosmology: Principles and Applications of the General Theory of Relativity (John Wiley and Sons, New York, 1972).

[81] R. K. Pathria, Statistical Mechanics (Butterworth-Heinemann, 2001).

[82] G. N. Watson, A Treatise on the Theory of Bessel Functions (Cambridge University Press, 1966).

[83] R. C. Tolman, Relativity, Thermodynamics and Cosmology (Dover, New York, 1987).

[84] F. Rohrlich, Classical Charged Particles (Addison-Wesley Publishing Inc., 1965).

[85] T. K. Nakamura, EuroPhys. Lett. 8840009 (2009). 
[86] N. G. van Kampen, Phys. Rev. 173, 295 (1968).

[87] F. Rohrlich, Nuovo Cim. 45B, 76 (1966); Am. J. Phys. 28, 639 (1960).

[88] R. Hakim y A. Mangeney, Lett. Nuovo Cim. 1, 429 (1969).

[89] A. Komar, Gen. Rel. Grav. 28, 379 (1996); P. T. Landsberg, Am. J. Phys. 60, 561 (1992); V. J. Menon and D. C. Agrawal, Am. J. Phys. 59, 258 (1991).

[90] D. Ter Haar and H. Wergeland, Phys. Rep. 1, 31 (1971).

[91] M. Mendoza, B. Boghosian, H. J. Herrmann and S. Succi, arxiv:0912.2913 (2009).

[92] L. B. Okun, Physics Today 31, 11 (1989), L. B. Okun, Sov. Phys. Usp. 32, 629 (1989).

[93] P. S. Faragó and L. Jánossy, Nuovo Cim. 51411 (1957);D. D. Meyerhofer, J. P. Knauer, S. J. McNaught and C. I. Moore, J. Opt. Soc. Am. B 13, 113 (1996); J. W. Luetzelschwab, Am. J. Phys. 71, 878 (2003); G. J. Aubrecht II, Am. J. Phys. 72, 970 (2004).

[94] D. J. Louis-Martinez, Class. Quantum Grav. 28, 035004 (2011).

[95] F. Becattinia, and L. Tinti, Annals of Physics, 325, 1566 (2010).

[96] E. A. Uehling and G. E. Uhlenbeck, Transport phenomena in Einstein-Bose and Fermi-Dirac gases I. Phys. Rev. 43, 552-561 (1933).

[97] I. Müller, T. Ruggeri, Extended Thermodynamics (Springer, New York, 1993).

[98] G. F. Smoot, M. V. Gorenstein and R. A. Muller, Phys. Rev. Lett. 39, 898 (1977); D. J. Fixen et al., Astrophys. J. 420, 445 (1994); D. J. Fixen et al., Astrophys. J. 473, 576 (1996).

[99] J. Alfaro and P. González, Int. J. Mod. Phys. D 17, 2171 (2008).

[100] S. S. Costa and G. E. A. Matsas, Phys. Lett. A 209, 155 (1995).

[101] P. T. Lansberg and G. E. A. Matsas, Phys. Lett. A 223, 401 (1996); P. T. Lansberg and G. E. A. Matsas, Physica A 340, 92 (2004).

[102] T. K. Nakamura, EuroPhys. Lett. 8820004 (2009).

[103] T. K. Nakamura, Phys. Lett. A 352175 (2006).

[104] Z. C. Wu, EuroPhys. Lett. 8820005 (2009).

[105] G. Chacón-Acosta and G. M. Kremer, Fokker-Planck-type equations for a simple gas and for a semirelativistic Brownian motion from a relativistic kinetic theory. Phys. Rev. E 76, 021201 (2007). 
[106] T. K. Nakamura, EuroPhys. Lett. 8940007 (2010).

[107] D. Jou, J. Casas-Vázquez, G. Lebon, Extended Irreversible Thermodynamics (Springer, New York, 2010).

[108] V. A. Belinskiǐ, E. S. Nikomarov, and I. M. Khalatnikov, Sov. Phys. JETP 50, 213 (1979).

[109] L. Philpott, F. Dowker and R. D. Sorkin, Phys. Rev. D 79, 124047 (2009).

[110] J. Racker, P. Sisterna and H. Vucetich, Phys. Rev. D 80, 083526 (2009).

[111] S. Das, S. Ghosh and D. Roychowdhury, Phys. Rev. D 80, 125036 (2009).

[112] C. Marle, C. R. Acad. Sc. Paris 260, 6539 (1965).

[113] J. L. Anderson and H. R. Witting, Physica 74, 489 (1974).

[114] P. Bhatnagar, E. Gross and M. Krook, Phys. Rev. 94, 511 (1954).

[115] E. Bertschinger, Albert Einstein Century International Conference. AIP Conf. Proc. 861, 97-105 (2006).

[116] R. Kuiper and G. Wolschin, Ann. Phys. (Leipzig) 16, No. 1, 67 - 77 (2007).

[117] J. Dunkel and P. Hänggi, Relativistic Brownian Motion, Phys. Rep. (2009)

[118] F. Debbasch, Albert Einstein Century International Conference. AIP Conf. Proc. 861, 488-493 (2006).

[119] C. S. Wang Chang and G. E. Uhlenbeck, in Studies in Statistical Mechanics Vol. IV, ed. J. de Boer and G.E. Uhlenbeck, 76-100 (1970).

[120] M. S. Green, J. Chem. Phys. 19, 1036-1046 (1951).

[121] R. Brown, Philos. Mag. 4, 161-173 (1828), reimpreso en Edinburgh New Philos. J. 5, 358-371, (1928).

[122] A. Einstein, Investigations on the Theory of the Brownian Movement. Dover Publications, Inc. (1956).

[123] M. von Smoluchowski, Zur kinetischen Theorie der Brownschen Molekularbewegung und der Suspensionen, Ann. Physik (Leipzig) 21, 756 (1906).

[124] J. Perrin. Ann. Chim. Phys. 18, 5-114 (1909).

[125] P. Hänggi and F. Marchesoni, Chaos 15 (2005), 026101. 
[126] D. S. Lemons, An Introduction to Stochastic Processes in Physics. The John Hopkins University Press (2002).

[127] F. Reif, Fundamentals of Statistical and Thermal Physics. McGraw-Hill Inc. (1965).

[128] H. A. Lorentz, Proc. Amst. Acad. 7, 438, 585, 684 (1905).

[129] S. Chapman and T. G. Cowling, The Mathematical Theory of Non-Uniform Gases. 3th ed. Cambridege University Press, Cambridge (1970).

[130] K. Andersen and K. E. Shuler, J. Chem. Phys. 40, 633 (1964).

[131] H. Oser, K. E. Shuler and G. H. Weiss, J. Chem. Phys. 41, 2661 (1964).

[132] K. D. Knierim, M. Waldman and E. A. Mason, J. Chem Phys. 77, 943 (1982).

[133] D. C. Kelly, Phys. Fluids 12, 799 (1969).

[134] A. J. Kox, Phys. Lett A 53, 173 (1975); A. J. Kox, Physica A 84, 603-882 (1976).

[135] D. Mosher, Phys. Fluids 18, 846 (1979).

[136] C. Cercignani, The Boltzmann Equation and Its Appliacations. (Springer-Verlag, New York, 1988).

[137] E. M. Lifshitz and L. P. Pitaevskii, Physical Kinetics. (Pergamon Press, Oxford, 1981).

[138] H. Akama, J. Phys. Soc. Japan 28 (2), 478-488 (1970).

[139] C. W. Gardiner, Handbook of Stochastic Methods for Physics Chemistry and the Natural Sciences. Springer (2004).

[140] H. Risken, The Fokker-Planck Equation Methods of Solution and Applications. Springer (1996).

[141] J. A. Alcántara-Félix, arXiv:1011.5429.

[142] C. F. Lo, Europhys. Lett. 39, 263-267 (1997).

[143] V. M. Kenkre and M. N. Kuperman, Phys. Rev. E 67, 051921 (2003).

[144] K. Zabrocki, S. Tatur, S. Trimper, R. Mahnke, Phys. Lett. A 359, 349-356 (2006); M. Schulz, S. Trimper and K. Zabrocki, J. Phys. A: Math. Theor. 40 3369-3378 (2007).

[145] S. Trimper and K. Zabrocki, Phys. Lett. A 331, 423-431 (2004).

[146] A. Macías and L. Dagdug (Eds.), New Trends in Statistical Physics: Festschrift in Honor of Leopoldo García-Colin's 80th Birthday, World Scientific, (2010). 
[147] C. Rovelli, Quantum Gravity, Cambridge Monographs on Mathematical Physics. Cambridge (2004).

[148] T. Thiemann, Modern Canonical Quantum General Relativity . Cambridge University Press (2007).

[149] M. Bojowald, Loop Quantum Cosmology, Liv. Rev. Rel. 4, 11 (2008). http://www.livingreviews.org/lrr-2008-4.

[150] A. Ashtekar, T. Pawlowski and P. Singh, Quantum nature of the big bang: Improved dynamics. Phys. Rev. D 74, 084003 (2006).

[151] M. Bojowald, Absence of a Singularity in Loop Quantum Cosmology. Phys. Rev. Lett. 86, 5227-5230 (2001).

[152] M. Bojowald, Quantum nature of cosmological bounces. Gen. Rel. Grav. DOI:10.1007/s10714-0080645-1 (2008).

[153] C. Rovelli, Black Hole Entropy from Loop Quantum Gravity. Phys. Rev. Lett. 77 3288-3291 (1996).

[154] A. Ashtekar, J. Baez, A. Corichi and K. Krasnov, Quantum Geometry and Black Hole Entropy. Phys. Rev. Lett. 80 904-907 (1998).

[155] M. Domagala and J. Lewandowski, Black-hole entropy from quantum geometry. Class. Quantum Grav. 21 5233-5243 (2004).

[156] J. J. Halliwell and S. W. Hawking, Origin of structure in the Universe. Phys. Rev. D 31 1777-1791 (1985).

[157] L. P. Grishchuk and Y. V. Sidorov, Relic graviton and the birth of the universe. Class. Quantum Grav. 6 L155-L160 (1989).

[158] L. P. Grishchuk and Y. V. Sidorov, Squeezed quantum states of relic gravitons and primordial density fluctuations. Phys. Rev. D 42 3413-3421 (1990).

[159] M. Bojowald, Inflation from Quantum Geometry. Phys. Rev. Lett. 89261301 (2002).

[160] S. Tsujikawa, P. Singh and R Maartens, Loop quantum gravity effects on inflation and the CMB. Class. Quantum Grav. 21 5767-5775 (2004).

[161] J. Mielczarek and M. Szydłowski, Relic gravitons as the observable for loop quantum cosmology. Phys. Lett. B 657 20-26 (2007).

[162] J. Mielczarek and M. Szydłowski, Relic gravitons from super-inflation, gr-qc/0710.2742 (2007).

[163] M. Bojowald and G. M. Hossain, Loop quantum gravity corrections to gravitational wave dispersion. Phys. Rev. D 77, 023508 (2008). 
[164] J. Mielczarek, Gravitational waves from the Big Bounce, gr-qc/0807.0712 (2008).

[165] A. Ashtekar, S. Fairhurst and J. L. Willis, Quantum gravity, shadow states and quantum mechanics. Class. Quantum Grav. 20 1031-1061 (2003).

[166] A. Corichi, T. Vukašinac and J. A. Zapata, Hamiltonian and physical Hilbert space in polymer quantum mechanics. Class. Quantum Grav. 24 1495-1511 (2007).

[167] A. Corichi, T. Vukašinac and J. A. Zapata, Polymer quantum mechanics and its continuum limit. Phys. Rev. D 76044016 (2007).

[168] V. Hussain, J Louko and O. Winkler, Quantum gravity and the Coulomb potential. Phys. Rev. D 76, 084002 (2007).

[169] K. Fredenhagen and F. Reszewski, Polymer state approximation of Schrödinger wave functions. Class. Quantum Grav. 23 6577-6584 (2006).

[170] J. M. Velhinho, The quantum configuration space of loop quantum cosmology. Class. Quantum Grav. 24, 3745-3758 (2007).

[171] Dah-Wei Chiou, Galileo symmetries in polymer particle representation. Class. Quantum Grav. 24 $2603-2620$ (2007).

[172] G. Kunstatter, J. Louko and J. Ziprick, Polymer quantization, singularity resolution, and the $1 / r^{2}$ potential. Phys. Rev. A 79, 032104 (2009).

[173] G. Amelino-Camelia, Introduction to Quantum-Gravity Phenomenology. gr-qc/0412136 (2004).

[174] G. Amelino-Camelia, Quantum Gravity Phenomenology. 0806.0339 (2008).

[175] G. Amelino-Camelia, J. Ellis, N. E. Mavromatos, D. V. Nanopoulos and S. Sarkar Tests of quantumgravity from observations of gamma-ray bursts. Nature 393763 (1998).

[176] T. J. Konopka and S. A. Major Observational limits on quantum geometry effects. New J. Phys. 4, 57.1-57.18 (2002).

[177] J. Alfaro, H. A. Morales-Técotl and L. F. Urrutia, Quantum gravity corrections to neutrino propagation. Phys. Rev. Lett. 842318 (2000).

[178] J. Alfaro, H. A. Morales-Técotl and L. F. Urrutia, Loop quantum gravity and light propagation. Phys. Rev. D 65103509 (2002).

[179] A. Camacho, Generalized Uncertainty Principle and Deformed Dispersion Relation Induced by Nonconformal Metric Fluctuations. Gen. Rel. Grav. 341839 (2002). 
[180] A. Camacho, Generalized Uncertainty Principle and Quantum Electrodynamics. Gen. Rel. Grav. 35 1135 (2002).

[181] A. Kempf, G. Mangano and R. B. Mann, Hilbert space representation of the minimal lenght uncertainty relation. Phys. Rev. D 521108 (1995).

[182] H. Hinrichsen and A. Kempf, Maximal localization in the presence of minimal uncertainties in position and in momenta. J.Math.Phys. 37 2121-2137 (1996).

[183] M. Reed and B. Simon, Methods of Modern Mathematical Physics. Vol. I Functional Analysis. Academic Press Inc. London (1980).

[184] A. Ashtekar, A. Corichi and P. Singh, Robustness of key features of loop quantum cosmology. Phys. Rev. D 77, 024046 (2008).

[185] K. Nozari and T. Azizi, Some aspects of gravitational quantum mechanics. Gen. Rel. Grav. 38 $735-742(2006)$.

[186] S. Elaydi, An Introduction to Difference Equations. Springer, New York, Inc. (1996).

[187] J. Swain, Exotic Statistics for Ordinary Particles in Quantum Gravity. IJMPD 17, 2475 (2008).

[188] L. García-Colín Scherer, La naturaleza estadística de la teoría de los cuantos. Colección CBI, Universidad Autónoma Metropolitana Unidad Iztapalapa (1987).

[189] H. Yépez, J. M. Romero and A. Zamora, Corrections to the Planck's radiation law from loop quantum gravity. hep-th/0407072, (2004).

[190] K. Nozari and A. S. Sefidgar, The effect of modified dispersion relations on the thermodynamics of black-body radiation. Chaos Solitons \& Fractals 38 339-347 (2008).

[191] A. Camacho and A. Macías, Thermodynamics of a photon gas and deformed dispersion relations. Gen. Rel. Grav. 39 1175-1183 (2007).

[192] G. A. Baker Jr., Quantitative Theory of Critical Phenomena. Academic Press Inc. (1990).

[193] K. Nozari and H. Mehdipour, Implications of minimal length scale on the statistical mechanics of ideal gas. Chaos Solitons \& Fractals 32 1637-1644 (2007).

[194] D. Colladay and P. McDonald, Statistical Mechanics and Lorentz Violation. Phys.Rev. D 70125007 (2004).

[195] D. Colladay and V. A. Kostelecký, CPT violation and the standard model. Phys. Rev. D 55, 6760 (1997). 
[196] D. Colladay and V. A. Kostelecký, Lorentz-violating extension of the standard model. Phys. Rev. D 58, 116002 (1998).

[197] K. Nozari, B. Fazlpour, Generalized uncertainty principle, modified dispersion relations and the early universe thermodynamics. Gen. Rel. Grav. 38 1661-1679 (2006).

[198] T. Konopka, F. Markopoulou and S. Severini, Quantum graphity: A model of emergent locality. Phys. Rev. D 77, 104029 (2008).

[199] T. Konopka, Statistical mechanics of graphity models. Phys. Rev. D 78, 044032 (2008).

[200] L. H. Kauffman, Knots and Statistical Mechanics. Chaos Solitons \& Fractals 9599 (1998).

[201] K. Ø. Rasmussen, T. Cretegny, P.G. Kevrekidis and N. Grøbench-Jensen, Statistical Mechanics of a Discrete Nonlinear System. Phys. Rev. Lett. 84, 3740 (2000).

[202] V. H. Hamity and D. E: Barraco, Generalized Nonextensive Thermodynamics Applied to the Cosmic Background Radiation in a Robertson-Walker Universe. Phys. Rev. Lett. 76, 4664 (1996).

[203] C. Rovelli and S. Speziale, Reconcile Planck-scale discreteness and the Lorentz-Fitzgerald contraction. Phys. Rev. D 67064019 (2003).

[204] M. Bojowald, H. A. Morales-Técotl and H. Sahlmann, Loop quantum gravity phenomenology and the issue of Lorentz invariance. Phys Rev. D 71, 084012 (2005).

[205] E. Manrique, R. Oeckl, A. Weber and J. A. Zapata, Loop quantization as a continuum limit. Class. Quantum Grav. 23 3393-3403 (2006).

[206] F. Debbasch and W. A. van Leeuwen, General relativistic Boltzmann equation, I: Covariant treatment, Physica A 388 1079-1104, (2009); General relativistic Boltzmann equation, II: Manifestly covariant treatment, Physica A 388 1818-1834, (2009).

[207] C. J. Fewster and H. Sahlmann, Phase space quantization and loop quantum cosmology: a Wigner function for the Bohr-compactified real line, Class. Quantum Grav. 25225015 (2008).

[208] A. P. Balachandran, G. Marmo, N. Mukunda, J. S. Nilsson, A. SimoniE. C. G. Sudarshan and F. Zaccaria, J. Math. Phys. 25, 167 (1984).

[209] M. H. L. Pryce, Proc. R. Soc. Lond. A 19562 (1948).

[210] R. M. F. Houtappel, H. van Dam and E. P. Wigner, Rev. Mod. Phys. 37, 595 (1965).

[211] F. Coester and P. Havas, Phys. Rev. D 14, 2556 (1976).

[212] N. Mukunda and E. C. G. Sudarshan, Phys. Rev. D 23, 2210 (1981). 
[213] N. Mukunda, E. C. G. Sudarshan and J. N. Goldberg, Phys. Rev. D 23, 2218 (1981).

[214] A. Komar, Phys. Rev. D 18, 1887 (1978).

[215] G. B. Arfken and H. J. Weber, Mathematical Methods for Physicists Sixth Edition. ELsevier Academic Press (2005).

[216] C. Isham, Conceptual and Geometrical Problems in Quantum Gravity in H. Mitter and H. Gausterer (eds.) Recent Aspects of Quantum Fields pp. 123-230, Springer-Verlag (1992).

[217] C. Isham, Prima Facie Questions in Quantum Gravity. gr-qc/9310030 (1993).

[218] C. Isham, Structural Issues in Quantum Gravity. gr-qc/9510063 (1995).

[219] J. Polchinski, String Theory Vol.1 y 2, Cambridge University Press. Cambridge (1998).

[220] B. L. Hu, Quantum Gravity and the origin of the Universe. gr-qc/0611058 (2006).

[221] B. L. Hu, General Relativity as Geometro-Hydrodynamics. gr-qc/9607070, (1996).

[222] B. L. Hu, A Kinetic Theory Approach to Quantum Gravity. Int. J. Theor. Phys. 41 2091-2119 (2002).

[223] B. L. Hu and E. Verdaguer, Stochastic gravity: a primer with applications, Class. Quantum Grav. 20 R1-R42 (stacks.iop.org/CQG/20/R1), (2003).

[224] L. H. Ford, Stochastic Spacetime and Brownian Motion of Test Particles. Int. J. Theor. Phys. 44 1753-1768 (2005).

[225] L. H. Ford, Spacetime in Semiclassical Gravity, (pág. 293) 100 Years of Relativity. Space-Time Structure: Einstein and Beyond, A. Ashtekar (Ed.) World Scientific Publishing Co. Pte. Ltd. (2005).

[226] G. Amelino-Camelia, A perspective on Quantum Gravity Phenomenology. astro-ph/0402009, (2004).

[227] G. Amelino-Camelia, C. Lämmerzahl, A. Macias and H. Müller, The Search of Quantum Gravity Signals. gr-qc/0501053 (2005).

[228] F. Dowker, Causal sets and the deeep structure of spacetime, (pág. 445) 100 Years of Relativity. Space-Time Structure: Einstein and Beyond, A. Ashtekar (Ed.) World Scientific Publishing Co. Pte. Ltd. (2005).

[229] A. Pérez, Loop quantum gravity. Europhysicsnews 37, 17 (2006).

[230] A. Pérez, Introduction to Loop Quantum Gravity and Spin Foams. Proc. of the II Int. Conference on Fundamental Interactions, Pedra Azul, Brazil, gr-qc/0409061 (2004). 
[231] A. Corichi y D. Nuñez, Introducción al formalismo ADM. Rev. Mex. Fís. 37 720-747 (1991).

[232] M. Bojowald, Loop Quantum Cosmology, (pág. 382) 100 Years of Relativity. Space-Time Structure: Einstein and Beyond, A. Ashtekar (Ed.) World Scientific Publishing Co. Pte. Ltd. (2005).

[233] M. Bojowald, What happened before the Big Bang? Nature Physics 3, 523-525 (2007).

[234] M. Bojowald, Harmonic cosmology: How much can we know about a universe before the big bang? gr-qc/0710.4919, (2007).

[235] A. Corichi and P. Singh, Quantum bounce and cosmic recall. gr-qc/0710.4543, (2007).

[236] J. Mielczarek, T. Stachowiak and M. Szydłowski, Exact solutions for Big Bounce in loop quantum cosmology. gr-qc/0801.0502, (2008).

[237] J. Mielczarek and M. Szydłowski, $\mathcal{O}\left(\mu^{4}\right)$ corrections from holonomies in Loop Quantum Gravity and its effect on flat FRW models. gr-qc/0801.1073, (2008).

[238] A. Corichi, T. Vukašinac and J. A. Zapata, On a Continuum Limit for Loop Quantum Cosmology. Proceedings of the Third Mexican Meeting on Mathematical and Experimental Physics, gr-qc/0711.0788, (2007). 